\title{
An analysis of spindle microtubule interactions mediated by the Ndc80 complex, Cep57R and Cep57
}

John Gerald Tooley, Jr.

Syracuse, New York

B.A. Biology, University of Rochester, 2000

Master of Science, University of Virginia, 2008

A Dissertation presented to the Graduate Faculty of the University of Virginia in Candidacy for the Degree of

Doctor of Philosophy

Department of Cell Biology

University of Virginia

August, 2011

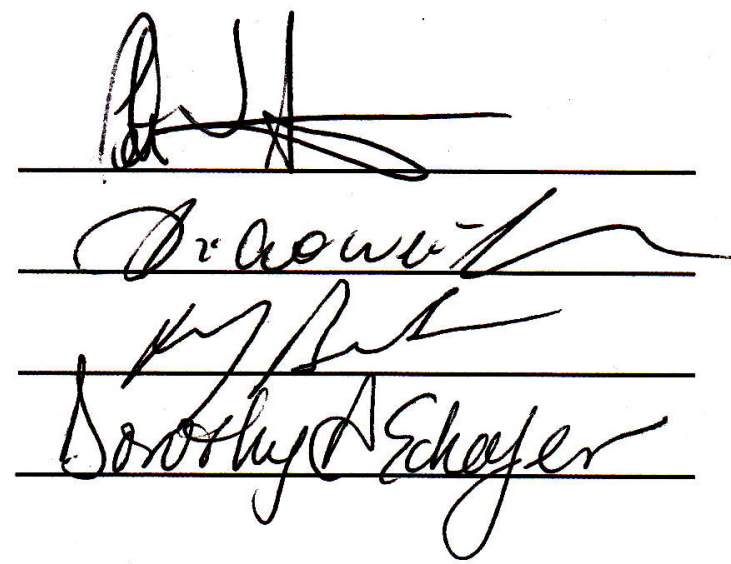




\section{Abstract}

During mitosis, kinetochores link centromeric DNA to spindle microtubules to facilitate accurate segregation of the replicated genome. Spindle poles also must form stable microtubule attachments to generate the opposing forces required for the depolymerization-coupled movement of chromosomes during anaphase. Kinetochores contain between $80-100$ proteins, but the mechanism by which this macromolecular structure couples to microtubules remains unknown. Models invoking a kinetochore sleeve, a sliding ring and a fibrillar coupler have been proposed to explain how the kinetochore harnesses the energy released by a depolymerizing microtubule to power chromosome movements. In this dissertation we test two of these models through extensive study of two proteins thought to function as key kinetochore couplers.

Chapter 1 provides a general introduction to mitosis, kinetochores and microtubules. Chapter 2 tests whether $\mathrm{xCep57R}$ is a functional vertebrate homolog of Dam1, a budding yeast protein that forms a sliding ring on microtubules. Chapter 3 provides an extensive review of $\mathrm{Ndc} 80$ complex function, while Chapter 4 identifies a tripartite attachment point in the Hec1/Ndc80 subunit that allows the $\mathrm{Ndc} 80$ complex to couple to microtubules. Chapter 5 concludes the dissertation by providing data suggesting that the unstructured tail of Hec1/Ndc80 facilitates chromosome congression by packing Ndc80 complexes close together and enhancing kinetochore processivity on a depolymerizing microtubule. Chapter 5 finishes with a section detailing future directions for more precisely determining the coupling mechanism of the vertebrate $\mathrm{Ndc} 80$ complex. 


\section{Table of Contents}

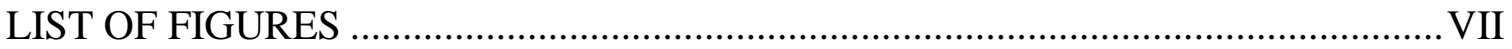

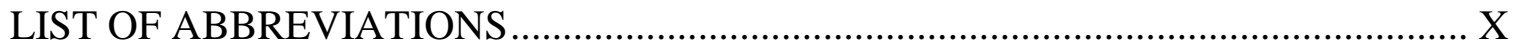

CHAPTER 1: GENERAL INTRODUCTION...................................

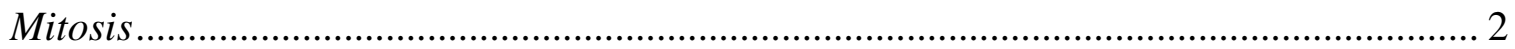

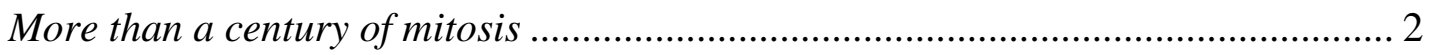

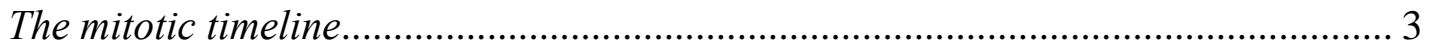

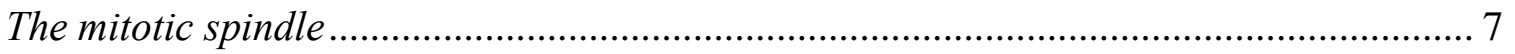

Centrosomes/spindle poles........................................................................... 7

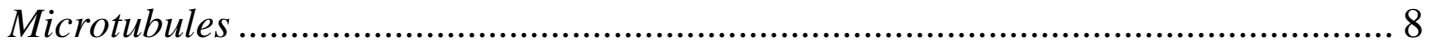

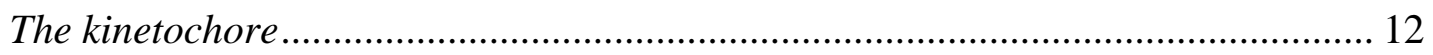

Kinetochore structure ........................................................ 12

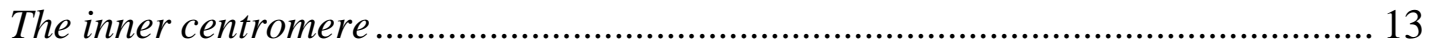

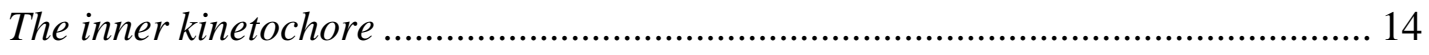

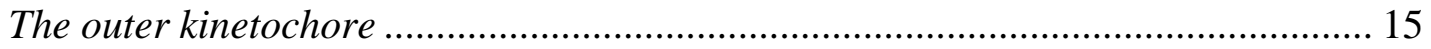

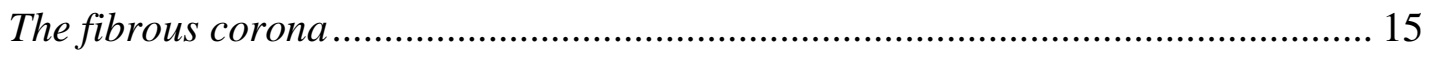

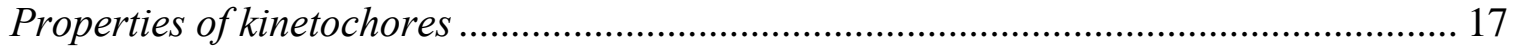

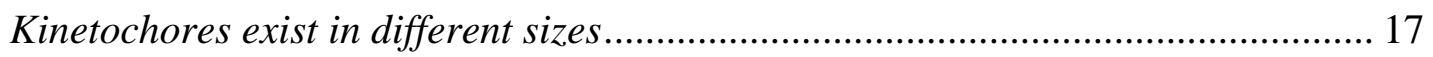

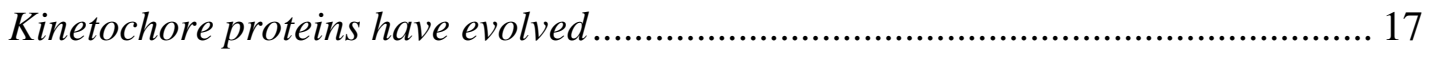

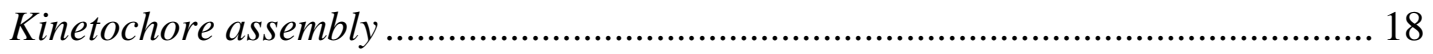

The dynamics of kinetochore-microtubule interactions .............................................. 20

Kinetochores perform work on microtubules .................................................... 20

Microtubules perform work on kinetochores ..................................................... 21 
Models for kinetochore-microtubule attachment ............................................... 22

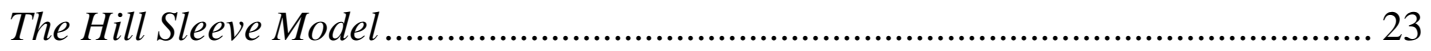

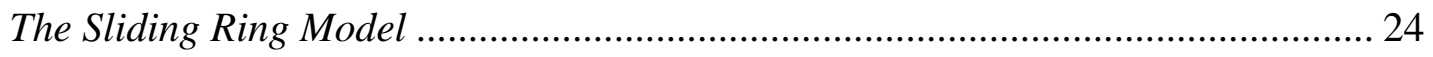

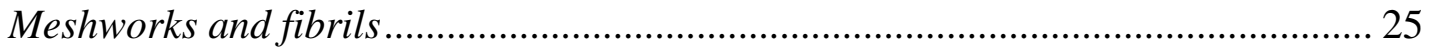

Preventing improper mitosis - and the consequences of failure ................................... 29

Detecting and repairing improper kinetochore-microtubule attachments .............. 29

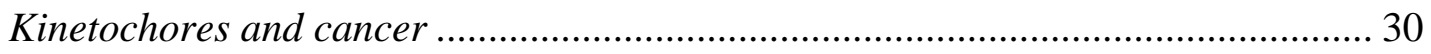

\section{CHAPTER 2: STRUCTURE-FUNCTION AND PHENOTYPIC ANALYSIS OF THE} CENTROSOME PROTEINS CEP57R AND CEP57 .............................. 31

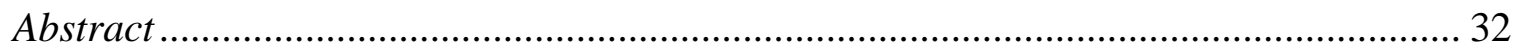

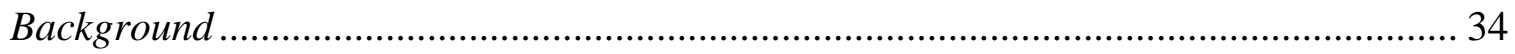

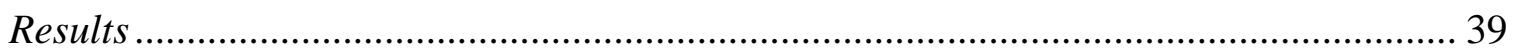

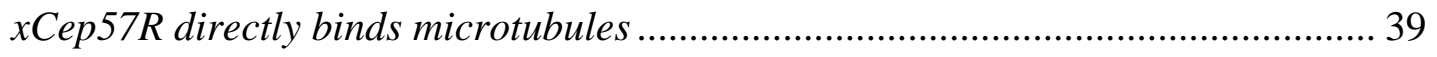

$x C e p 57 R$ does not form rings around microtubules .......................................... 42

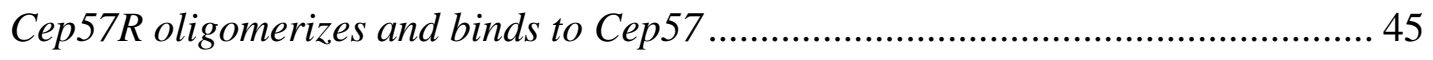

Cep57R and Cep57 interact with distinct sets of mitotic proteins .......................... 49

Cep57R and Cep57 are in vitro kinase substrates .............................................. 53

xCep57R and HURP are functionally separable .............................................. 56

Characeterization of $h C e p 57 R$ and $h$ Cep57 antibodies ...................................... 57

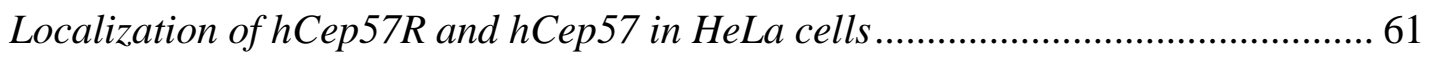

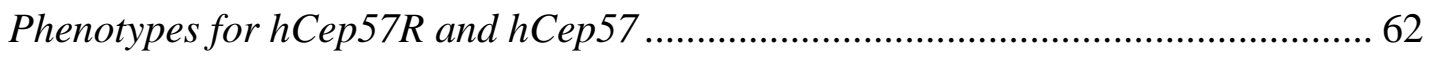

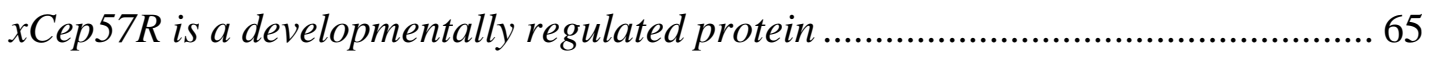

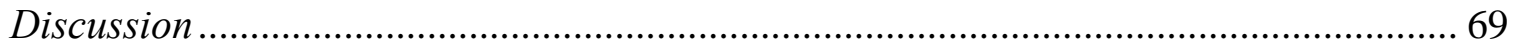


CHAPTER 3: THE NDC80 COMPLEX: INTEGRATING THE KINETOCHORE'S MANY MOVEMENTS ..................................................... 75

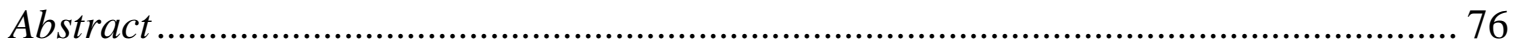

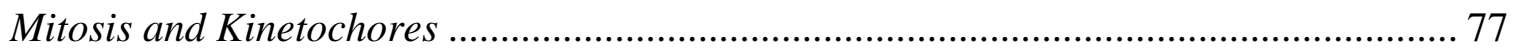

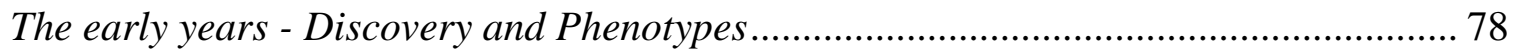

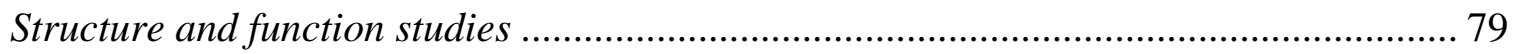

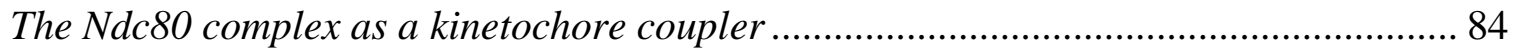

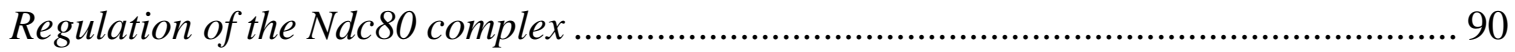

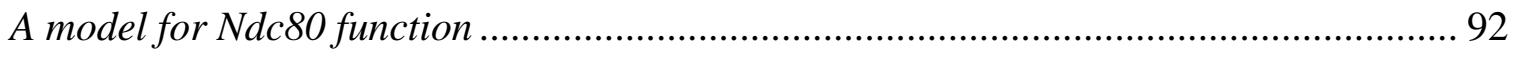

$N d c 80$ complex as an integrator of kinetochore function ........................................... 96

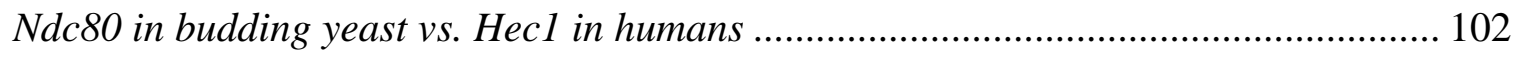

CHAPTER 4: THE NDC80 COMPLEX USES A TRIPARTITE ATTACHMENT POINT TO COUPLE MICROTUBULE ATTACHMENT TO CHROMOSOME MOVEMENT ..........................................................108

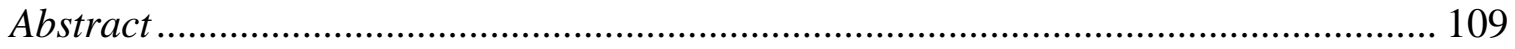

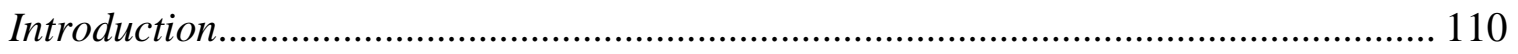

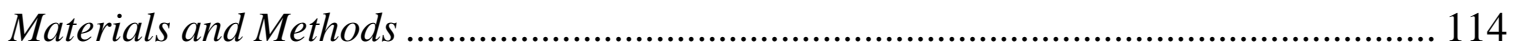

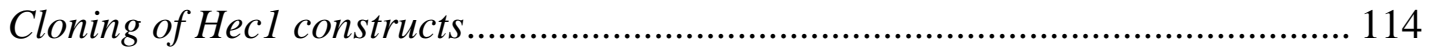

Recombinant Protein Expression and Purification .......................................... 114

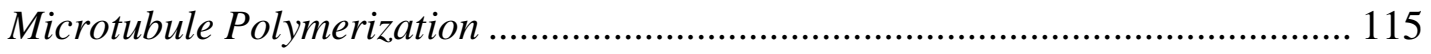

Western Blotting and microtubule cosedimentation assays................................ 115

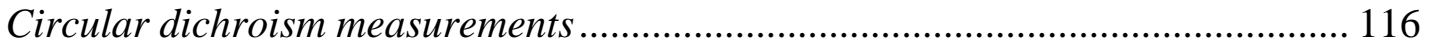

Knockdown and Rescue of Hec $1 /$ Ndc80 in HeLa cells ........................................ 116 


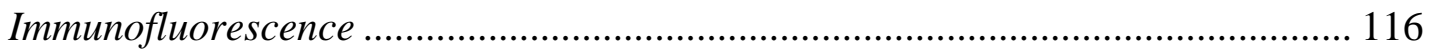

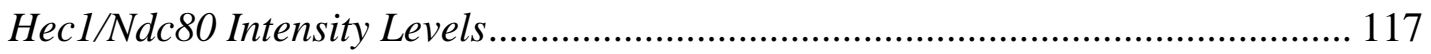

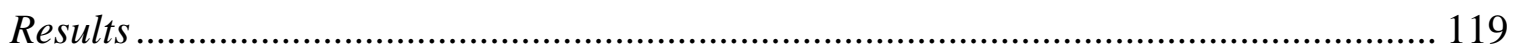

Cells expressing Hecl/Ndc80 CHD mutants assemble kinetochore properly........ 119

Kinetochores require the Hec 1/Ndc80 CHD to bind microtubules ........................ 123

CHD mutant phenotypes cannot be rescued by inhibiting Aurora B phosphorylation

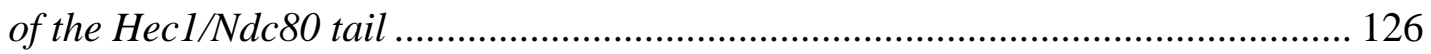

Both the Hecl/Ndc80 CHD and the N-terminal tail utilize charge to interact with

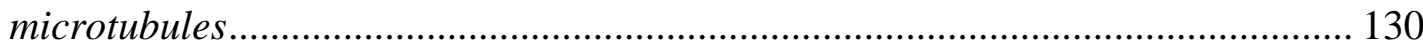

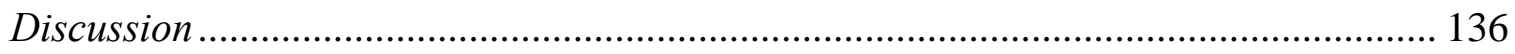

The Hecl/Ndc80 CHD has two distinct microtubule-binding motifs in vitro and both

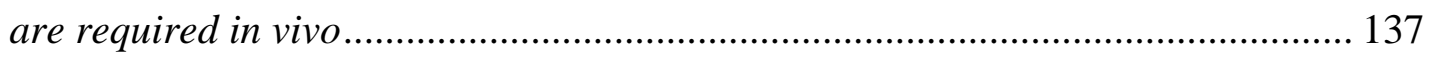

The role of the unstructured Hecl/Ndc80 tail ...................................................... 138

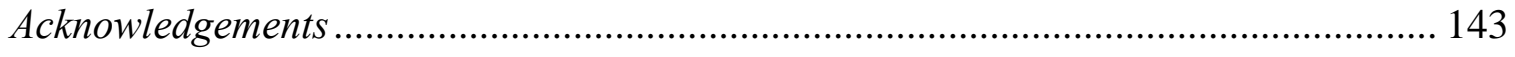

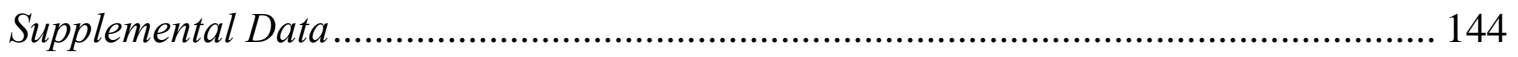

CHAPTER 5: ANALYSIS OF HEC1/NDC80 TAIL FUNCTION - DISTINCT

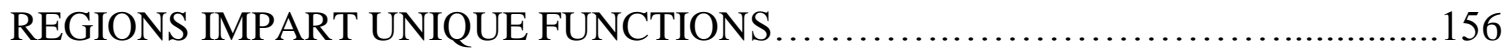

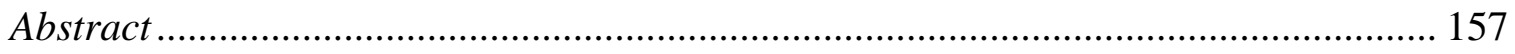

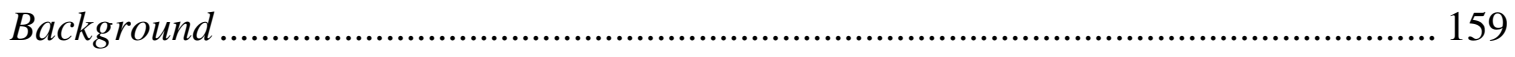

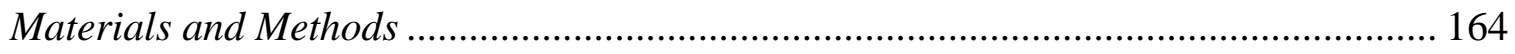

Recombinant Protein Expression and Purification .............................................. 164

Microtubule Polymerization and Co-Sedimentation Assays.................................. 164

Cloning of Hecl Constructs................................................................................ 164 


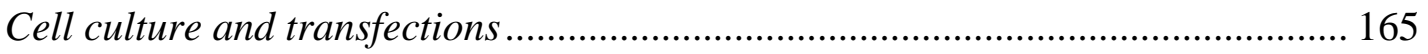

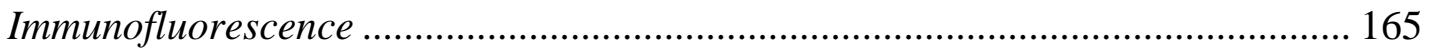

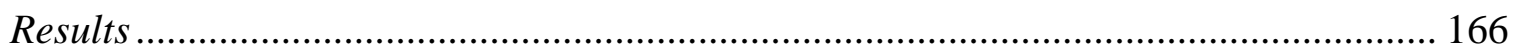

Binding properties of the Ndc80 complex are sensitive to changes in tail length and

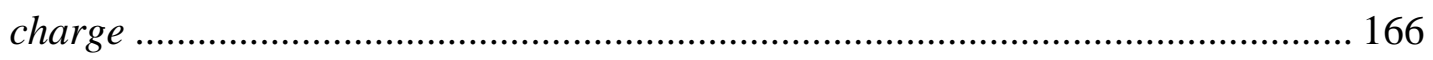

Minimal tail requirements for chromsome congression in vivo ........................... 174

Discussion and Future Directions ................................................................... 186

Affinity versus cooperativity ...................................................................... 186

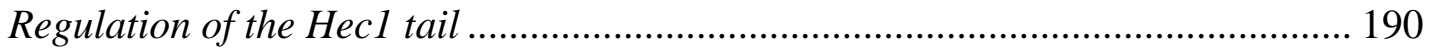

Cooperativity factors in vertebrates, yeast ................................................. 188

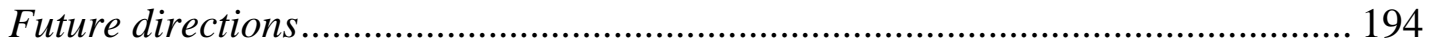

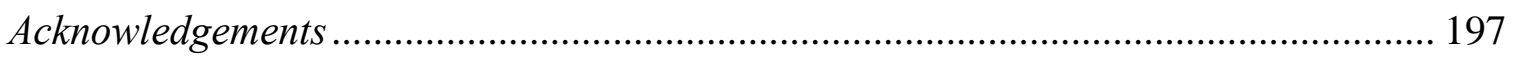

REFERENCES LIST .................................................. 198

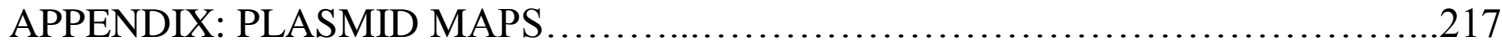




\section{List of Figures}

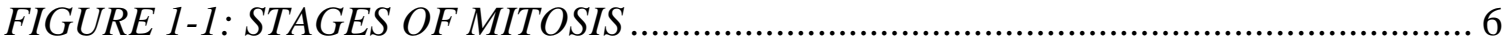

FIGURE 1-2: MICROTUBULE STRUCTURE AND DYNAMICS ............................... 11

FIGURE 1-3: MODELS FOR KINETOCHORE-MICROTUBULE COUPLING ............ 28

FIGURE 2-1: DOMAINS OF CEP57R AND SEQUENCE ALIGNMENTS FOR CEP57R

AND CEP57

FIGURE 2-2: FULL-LENGTH XCEP57R DIRECTLY BINDS MICROTUBULES ......... 41

FIGURE 2-3: EM IMAGES OF XCEP57R BOUND TO MICROTUBULES .................. 44

FIGURE 2-4: CEP57R OLIGOMERIZES AND DIRECTLY BINDS CEP57 IN VITRO . 48

FIGURE 2-5: CEP57 AND CEP57R INTERACT WITH NUMEROUS MITOTIC

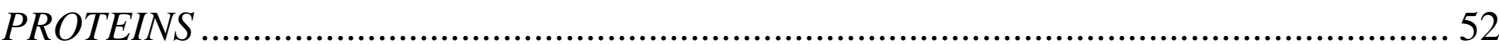

FIGURE 2-6: CEP57 AND CEP57R ARE PHOSPHORYLATED BY MITOTIC KINASES 55

FIGURE 2-7: CHARACTERIZATION OF HCEP57 AND HCEP57R 60

FIGURE 2-8: TESTING KINETOCHORE LOCALIZATION OF HCEP57R AND

HCEP57 64

FIGURE 2-9: CHARACTERISTICS OF XCEP57R DURING DEVELOPMENT. 68

FIGURE 3-1: STRUCTURE OF THE NDC8O COMPLEX. 83 
FIGURE 3-2: MODELS FOR DEPOLYMERIZATION-COUPLED MOVEMENT

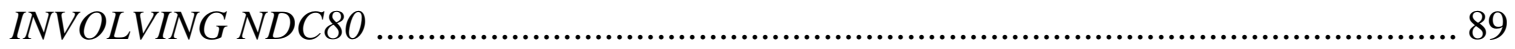

FIGURE 3-3: MODELS FOR DIFFERENTIAL NDC80/HEC1 TAIL

PHOSPHORYLATION 95

FIGURE 3-4: THE VERTEBRATE NDC8O COMPLEX INTEGRATED INTO THE OVERALL KINETOCHORE ARCHITECTURE. 101

FIGURE 3-5: THE BUDDING YEAST NDC80 COMPLEX INTEGRATED INTO THE OVERALL KINETOCHORE ARCHITECTURE. 107

FIGURE 4-1: THE CHD OF HEC1/NDC80 IS ESSENTIAL FOR CONGRESSION OF CHROMOSOMES TO THE METAPHASE PLATE

FIGURE 4-2: HEC1/NDC80 CHD MUTANT PHENOTYPES CANNOT BE RESCUED BY PREVENTING AURORA PHOSPHORYLATION OF THE HEC1/NDC80 TAIL .... 129

FIGURE 4-3: THE INTERACTION BETWEEN THE HEC1/NDC8O CHD AND A MICROTUBULE REQUIRES CHARGE.

FIGURE 4-4: POSITIVE CHARGE IN UNSTRUCTURED HEC1/NDC80 TAIL IS REQUIRED FOR MICROTUBULE BINDING \& CHROMOSOME CONGRESSION . 135 FIGURE 4-5: MODEL FOR VERTEBRATE HECI/NDC80'S INTERACTION WITH A MICROTUBULE 142 SUPP. FIGURE 4-1: CIRCULAR DICHROISM AND GEL FILTRATION OF HEC1/NDC80 CHD MUTANTS 
SUPP. FIGURE 4-2: CONTROLS DEMONSTRATING EFFECTIVENESS OF HEC1/NDC80 SIRNA AND REPLACEMENT PROTOCOL 147

SUPP. FIGURE 4-3: KINETOCHORES ASSEMBLE PROPERLY IN HEC1/NDC80 CHD MUTANT CELLS. 149

SUPP. FIGURE 4-4: INTER-KINETOCHORE DISTANCE MEASUREMENTS 151

SUPP. FIGURE 4-5: REPRESENTATIVE IMAGES OF MICROTUBULES FOLLOWING COLD TREATMENT 153

SUPP. FIGURE 4-6: KINETOCHORES ASSEMBLE PROPERLY IN HEC1 NEU MUTANT CELLS 155

FIGURE 5-1: THE HEC1 N-TERMINAL TAIL IS COMPRISED OF TWO BASIC REGIONS THAT CONTAIN AURORA B REGULATORY SITES 163

FIGURE 5-2: PURIFICATION OF HEC1 TAIL TRUNCATION MUTANTS IN NDC80 BONSAI 171

FIGURE 5-3: MICROTUBULE BINDING CURVES FOR NDC80 BONSAI TAIL MUTANTS 173

FIGURE 5-4: THE N-TERMINAL REGION OF THE HEC1 TAIL IS REQUIRED FOR CHROMOSOME ALIGNMENT. 178

FIGURE 5-5: DISTINCT REGIONS OF THE HEC1 TAIL HAVE DIFFERENT ALIGNMENT PHENOTYPES. 183 


\section{List of Abbreviations}

ACA: anti-centromere antigen

APC/C: Anaphase Promoting Complex/Cyclosome

ATP: adenosine triphosphate

CCAN: Constitutive Centromere Associated Network

CD: circular dichroism

CENP: centromere protein

$\mathrm{CH}$ : C-terminal half

CHD: Calponin homology domain

ChIP: chromatin immunoprecipitation

CIN: chromosome instability

CPC: Chromosome Passenger Complex

CT: C-terminus of xCep57R

DNA: deoxyribonucleic acid

EGFP: enhanced green fluorescent protein

EM: electron microscopy

FL: Full Length

GAPDH: glyceraldehyde-3-phosphate dehydrogenase

GDP: guanosine diphosphate

GST: glutathione S-transferase

GTP: guanosine triphosphate

HURP: Hepatoma Upregulated Protein

kDa: kilo Dalton

K-fiber: kinetochore-microtubule fiber

KMN: KNL-1, Mis12 complex and Ndc80 complex 
kMT: kinetochore microtubule

KNL-1: Kinetochore Null-1

K-SHREC: kinetochore single-molecule high resolution colocalization

IgG: Immunoglobulin G

MAP: microtubule associated protein

MCAK: mitotic centromere associated kinesin

MS/MS: Tandem Mass Spectrometry

MT: microtubule

MTBD: Microtubule Binding Domain

MTOC: microtubule organizing center

NH: N-terminal half

NT: N-terminus of xCep57R

N-Tip: N-terminal region of Hec1/Ndc80 tail

PAGE: polyacrylamide gel electrophoresis

PCM: pericentriolar material

PP1: Protein Phosphatase 1

RZZ: Rod, ZW10 and Zwilch

SAC: Spindle Assembly Checkpoint

Ska: Spindle and kinetochore associated

SDS: sodium dodecyl sulfate

siRNA: small interfering ribonucleic acid

TD-60: Telophase Disc 60

WT: wild type 


\section{ChAPTER 1:}

GENERAL INTRODUCTION 


\section{Mitosis}

\section{More than a century of mitosis}

Fundamental questions regarding the origin of new cells and the transmission of hereditary traits have fascinated scientists for more than a century. Early pioneers such as Walter Flemming, Theodor Boveri, and Edmund Wilson used light microscopy to make important observations and predictions about the basic underpinnings of mitosis. Since their time, generations of scientists have worked to develop a deeper understanding of this critical cellular process.

Up until the late $19^{\text {th }}$ century, it was believed that new cells in an organism arose from "free cell formation". This implied that a new cell emerged as the product of intercellular material that had morphed into a cellular structure (Paweletz, 2001). This theory, put forth by Schleiden and Schwann in the middle of the $19^{\text {th }}$ century, misled scientists for decades. However, a new generation of late $19^{\text {th }}$ and early $20^{\text {th }}$ century cell biologists disproved this theory. In the process, they laid the foundation for the modern study of cell division.

Flemming was one of the first cytologists to observe and describe the stages of cell division (Figure 1-1). He is credited with coining the term mitosis and also developed a method for staining chromosomes. Additionally, he was one of the first scientists to use the terms prophase, metaphase and anaphase to describe the stages of mitosis. These terms are still used today. At the turn of the $20^{\text {th }}$ century, Boveri put forth his theory regarding the "individuality of chromosomes", which argued that chromosomes contained the material of heredity. He was one of the first scientists to observe multipolar mitoses, and in 1902 Boveri postulated that cancer could be driven by defective cell 
divisions that resulted in an uneven distribution of chromosomes. Edmund Wilson, a champion of Boveri's work, later extended Boveri's findings through his own research on genetic inheritance and sex chromosomes. His seminal book, "The Cell in Development and Inheritance," is largely credited with advancing the concepts of the cell nucleus and of chromosomes as the transmitters of genetic traits. Indeed, more than a century later, many of the ideas put forth by these early researchers still hold true.

\section{The mitotic timeline}

In metazoans, mitosis follows a defined progression of morphologically distinct stages. It begins with prophase, a period characterized by the condensation of chromatin into chromosomes, the migration of duplicated centrosomes to opposite sides of the cell, and the breakdown of the nuclear envelope. Disassembly of the nuclear envelope liberates many sequestered factors that are then used for specialized mitotic functions. The mitotic spindle is formed during prometaphase. Microtubules are nucleated by centrosomes and begin probing the cytoplasm for chromosomes to capture. Their targets on chromosomes are kinetochores, large macromolecular structures that assemble on centromeric chromatin during mitosis. Captured chromosomes then convert initial microtubule contacts into stable end-on attachments. This conversion process, referred to as maturation, drives the congression of chromosomes to the cell center. During metaphase, chromosomes are aligned into a compact structure referred to as the metaphase plate. Following alignment of the final chromosome on the metaphase plate the cell initiates a major transition. During this transition from metaphase to anaphase, cohesion between sister chromatids is lost but microtubule attachments persist. Through their connection to rapidly shrinking microtubules, newly separated chromatids are then 
segregated to opposite ends of the dividing cell. Further separation of the spindle poles and the establishment of the cytokinetic furrow occur later in anaphase. Telophase follows and is characterized by complete ingression of the cytokinetic furrow and physical separation of the cell into a pair of daughter cells, each with a full genetic complement. 
Figure 1-1: Stages of mitosis

(A) Walter Flemming's early drawings of the stages of mitosis. Starting at top left and moving downward, a cell condenses its replicated DNA, breaks down its nuclear envelope and begins attaching chromosomes to microtubules in prometaphase. At the bottom of the image is metaphase, where all chromosomes align at the metaphase plate in the center of the cell. Anaphase ensues, chromosomes are segregated and spindle poles move apart. Cytokinesis is shown near the top right. These drawings were published in 1888 as part of Flemming's work entitled "Cell Substance, Nucleus and Cell Division." Image adapted from (Paweletz, 2001). Reprinted with permission from Nature Publishing Group; License Number 2713190516831. 

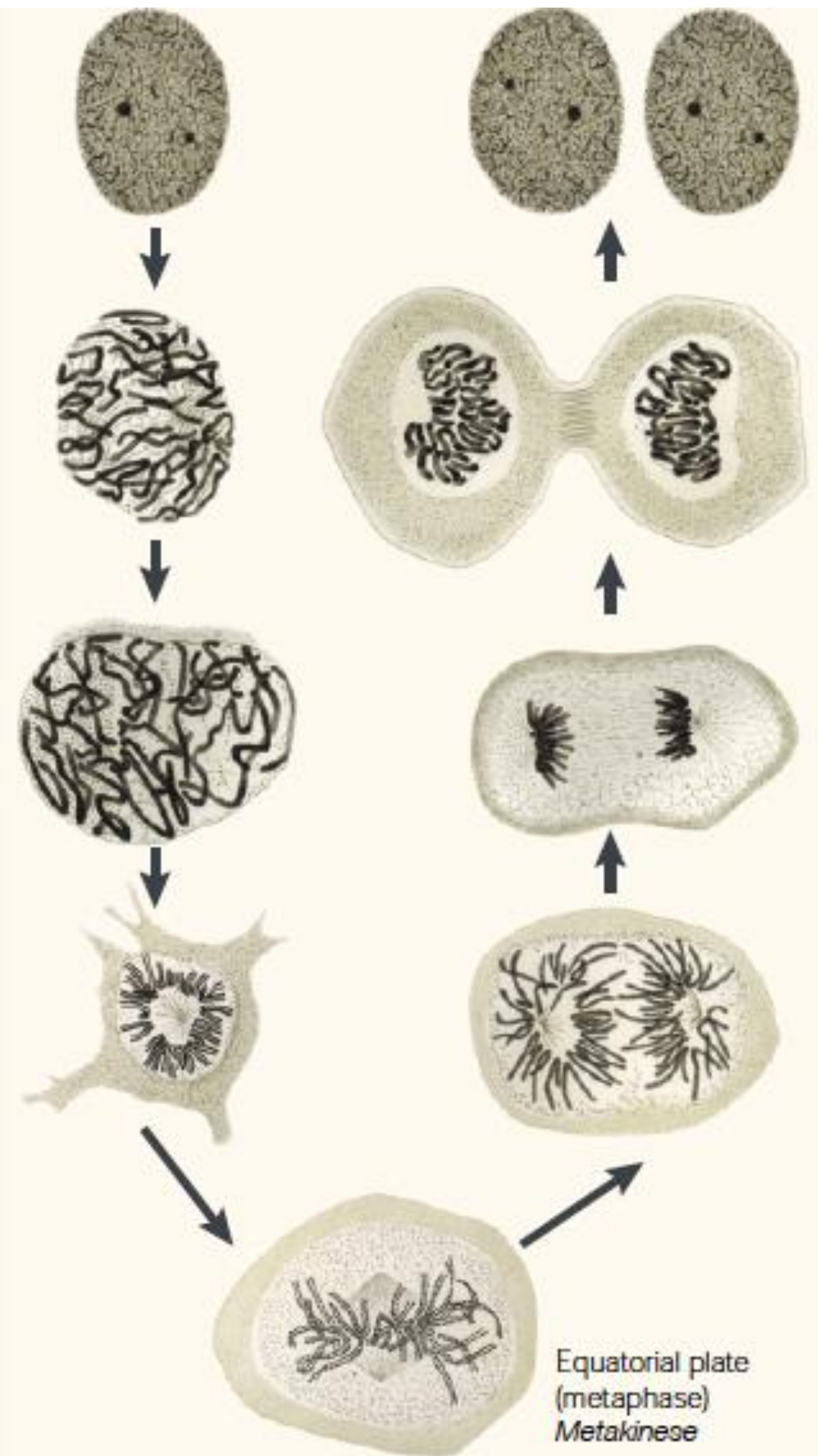

Figure 1-1 


\section{The mitotic spindle}

In vertebrate cells, the mitotic spindle is comprised of three main components: a pair of centrosomes, spindle microtubules and chromosomes. Assembly of this specialized, dynamic structure must occur in a relatively short time frame and involves numerous factors. Below I discuss the requirements for the assembly and function each of these three components.

\section{Centrosomes/spindle poles}

During a metazoan mitosis centrosomes serve as the microtubule organizing centers (MTOCs) for the cell. Centrosomes are composed of a pair of centrioles surrounded by an amorphous pericentriolar material (PCM) (Paintrand et al., 1992). The two centrioles are arranged perpendicular to one another and are comprised of proteins such as centrin and cenexin (Lange and Gull, 1995; White et al., 2000). The PCM surrounds the centrioles and contains a host of factors responsible for nucleating and focusing microtubules at the centrosome. These proteins include pericentrin, ninein and $\gamma$-tubulin (Andersen et al., 2003). Cep57 and Cep57R are also part of the PCM of vertebrate centrosomes, and will be discussed in detail in Chapter 2.

After undergoing duplication in S-phase, centrosomes separate and move to opposite ends of the cell during mitosis. Centrosomes nucleate, organize, and anchor spindle microtubules. These microtubules probe the cytoplasm of the cell in search of a chromosome to attach to. This dynamic process, called search-and-capture, was first described by Tim Mitchison and Marc Kirschner in 1986 (Kirschner and Mitchison, 1986). 


\section{Microtubules}

Microtubules form connections between chromosomes and spindle poles and power chromosome segregation during anaphase. Microtubules are dynamic polymers comprised of alternating $\alpha$ - and $\beta$-tubulin subunits. Tubulin subunits are mostly globular but also contain unstructured negatively charged tails (referred to as E-hooks) at their Ctermini. Subunits assemble end-to-end to form tubulin protofilaments, and a microtubule is comprised of 13 protofilaments arranged into a hollow, cylindrical tube. Tubulin polymers have an intrinsic polarity. The plus $(+)$ end of a microtubule ends with $\beta$-tubulin subunits, while the minus end of the microtubule always ends with $\alpha$-tubulin subunits. This intrinsic polarity causes a microtubule to have different rates of polymerization at its two ends, with the plus end polymerizing more rapidly. Tubulin monomers are GTPases that hydrolyze one molecule of GTP upon polymerization, but part of this hydrolysis energy is conserved in the polymer due to lateral constraints within the microtubule. Upon microtubule depolymerization, these constraints are lost, energy is released and a characteristic splaying of protofilaments occurs at the shrinking plus end (Figure 1-2). During microtubule polymerization, newly added tubulin subunits do not immediately hydrolyze their bound GTP. This delay results in a GTP "cap" which stabilizes the microtubule and promotes accelerated growth from this end.

Microtubules are dynamically unstable both in vivo and in vitro. First described by Mitchison and Kirschner in 1984, dynamic instability refers to the tendency of microtubules to rapidly shift between stages of growth and shrinkage (Mitchison and Kirschner, 1984). A growing plus end can suddenly switch to rapid depolymerization, an event referred to as a catastrophe. Conversely, a rapidly shrinking microtubule can 
suddenly transition back to a state of growth. This event is referred to as a rescue. In a relatively minimal in vitro system, microtubule dynamics are driven by changes in temperature, tubulin concentration, solution viscosity, and the abundance of free GTP (Andersen and Wittmann, 2002; Kristofferson et al., 1986; Mitchison and Kirschner, 1984). 
Figure 1-2: Microtubule structure and dynamics

(A) Cartoon of the atomic structure of $\alpha$ - and $\beta$-tubulin subunits. Subunits are incorporated into the microtubule in an alternating fashion. (B) Tubulin dimers dock onto the end of a polymerizing microtubule and induce GTP hydrolysis. (C) Due to constraints within the tubulin lattice, this energy from hydrolysis is conserved in the straightened polymer. A GTP cap (depicted in red) forms at the plus end of the microtubule due to a delay in GTP hydrolysis. (D) During microtubule depolymerization lattice restraints are lost, energy is released and the protofilaments adopt a curved conformation. This figure is adapted from (Howard and Hyman, 2003). Reprinted with permission from Nature Publishing Group; License Number 2713200296587. 

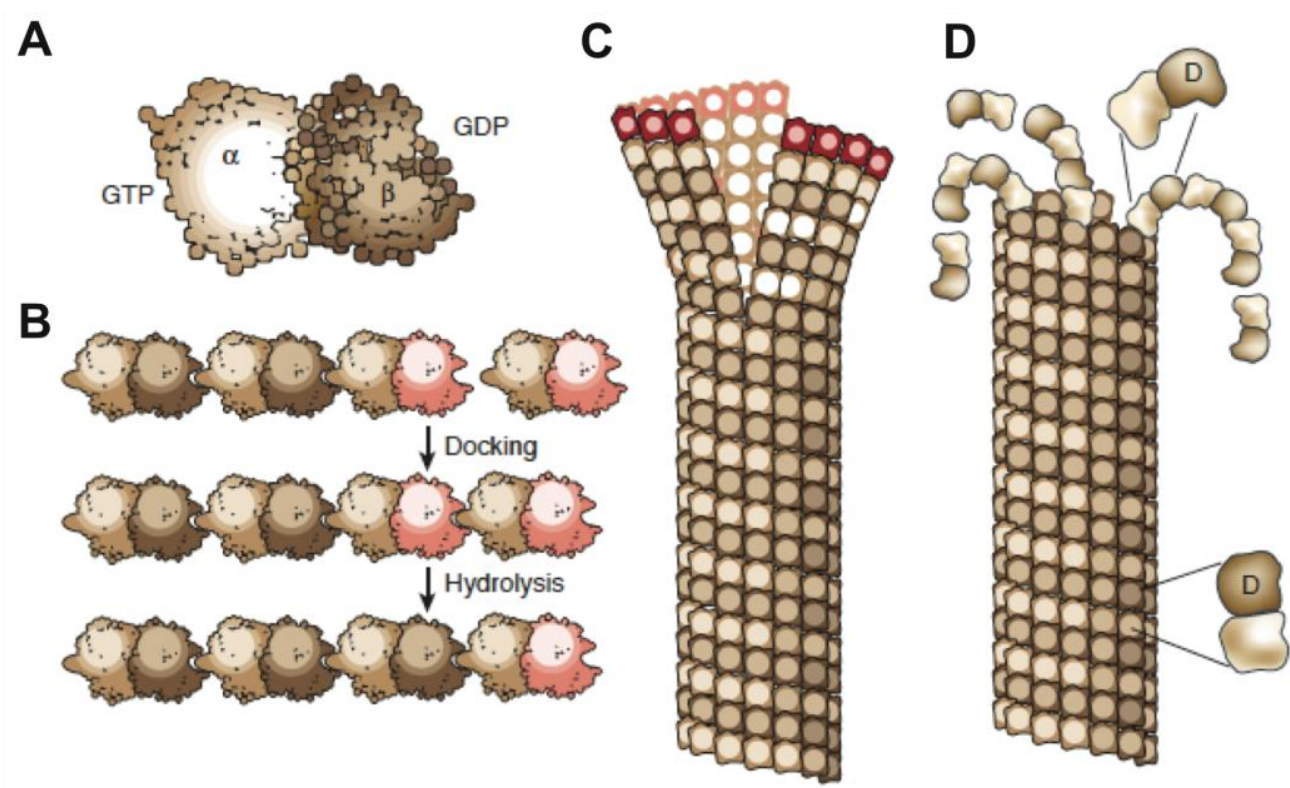

Figure 1-2 
In vivo, microtubule dynamics are not only affected by these parameters but also by the multitude of microtubule regulatory proteins that exist in a cell. The presence of these factors is thought to account for differences in measured microtubule dynamics in vivo compared to what is observed in vitro (Andersen and Wittmann, 2002; Kinoshita et al., 2001).

\section{The kinetochore}

The kinetochore is a macromolecular machine assembled on centromeric chromatin during mitosis. It serves as the link connecting replicated chromosomes to the spindle's segregation machinery. However, kinetochores are not simply microtubule binders. They also regulate the dynamics of bound microtubules, detect and correct improper attachments, and signal to the cell to halt mitotic progression until all chromosomes are properly attached to the spindle. Below, I discuss the composition of this unique cellular machine.

\section{Kinetochore structure}

The kinetochore, or "movement place", was identified and named by Lester Sharp and Franz Schrader in the 1930's (Sharp, LW 1934; Schrader, F 1939; Schrader, F 1944). Recognized as a central constriction on a chromosome where spindle microtubules attached, it was quickly appreciated that the kinetochore was able to facilitate chromosome movements in a cell. With the advent of electron microscopy (EM), scientists were able to glimpse the tri-laminar nature of the kinetochore structure (Brinkley and Stubblefield, 1966; Roos, 1973). The kinetochore sits atop centromeric chromatin (the inner centromere region) and contains a pair of electron dense layers 
(often referred to as the inner and outer plates), which are both $\sim 400 \AA$ thick. These two plates are separated by an electron-opaque middle layer which is $\sim 270 \AA$ thick. In the absence of microtubules, a region of fibrous material (named the fibrous corona) can be seen emanating from the outer plate (Mitchison and Kirschner, 1985). Following microtubule attachment, this region is no longer visible. The first molecular identification of kinetochore components came in the 1980's, with the identification of CENP-A, CENP-B and CENP-C in autoimmune serum from patients with a variant of the disease scleroderma (Earnshaw and Rothfield, 1985; Moroi et al., 1980). Around the turn of the $21^{\text {st }}$ century discovery of new kinetochore proteins came rapid-fire, and today the kinetochore parts list is thought to be nearly complete (Cheeseman and Desai, 2008). In vertebrates more than 80 kinetochore proteins have been identified. Furthermore, numerous cell biological and biochemical studies have placed many of these proteins into distinct regions of the kinetochore architecture (Welburn and Cheeseman, 2008).

\section{The inner centromere}

The inner centromere is defined as a heterochromatin-containing region residing between the two sister kinetochores. This region is responsible for maintaining cohesion between sister chromosomes during mitosis. The regulatory kinase Aurora B also resides in this region as part of a four-protein complex known as the Chromosome Passenger Complex (CPC). In addition to Aurora B, this complex also contains INCENP, Survivin and Borealin. The CPC localizes to the inner centromere from the onset on mitosis until the metaphase-to-anaphase transition (Carmena and Earnshaw, 2003; Vader et al., 2006). The spatial localization of the CPC - and its physical separation from many of its target substrates - is thought to control the timing of Aurora B regulation during mitosis (Liu et 
al., 2009). The inner centromere is also home to kinetochore proteins involved in microtubule destabilization (MCAK), Aurora B activation (TD-60) and regulatory kinases and phosphatases (Haspin, Plk1, PP2A) (Dai et al., 2005; Goto et al., 2006; Huang et al., 2007; Lan et al., 2004; Martineau-Thuillier et al., 1998).

\section{The inner kinetochore}

The inner kinetochore contacts the inner centromere and serves as the platform on which the remainder of the kinetochore assembles. Proteins in the inner kinetochore remain centromere-associated for nearly all stages of the cell cycle. The mitotic histone $\mathrm{H} 3$ variant CENP-A is thought to serve as the base of the inner kinetochore, and it has been shown to interact with centromeric chromatin as well as kinetochore proteins such as CENP-C and CENP-N (Carroll et al., 2010). Though discrepancies exist in the literature, a growing body of evidence also points to CENP-C having a role as a direct bridge between centromeric chromatin and the kinetochore (Hori et al., 2008). CENP-A and CENP-C belong to an extensive family of CENP proteins that are required for proper kinetochore assembly (Foltz et al., 2006; Hori et al., 2008; Okada et al., 2006). Due to their constitutive centromere localization, members of the Mis12 complex (Mis12, Dsn-1 Nnf-1 and Nsl-1) were initially designated as inner kinetochore proteins (Kline et al., 2006). However, its conserved interaction with outer kinetochore components such as KNL-1 and Ndc80 has now led to the Mis12 complex being considered an outer kinetochore component (Cheeseman et al., 2006). Recent studies linking Mis12 complex members with the CENP-C protein may reveal a connection point between the inner and outer kinetochore and explain this earlier confusion (Przewloka et al., 2011; Screpanti et 
al., 2011). CENP-T may also play a role in recruiting outer kinetochore components (Gascoigne et al., 2011).

\section{The outer kinetochore}

Following nuclear envelope breakdown, the outer kinetochore assembles on the inner kinetochore and is present through late anaphase. This region of the kinetochore is responsible for forming stable, end-on attachments to spindle microtubules, congressing chromosomes to form a metaphase plate and signaling the spindle checkpoint. The KMN network, a conserved group of proteins including $\underline{\mathrm{KNL}}-1$, the four-member $\underline{\text { Mis } 12}$

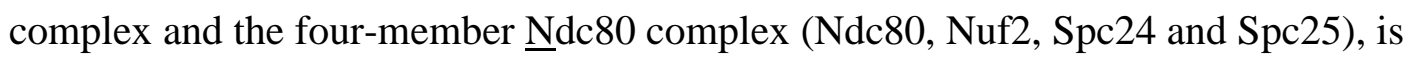
thought to serve as the primary attachment point for end-on microtubule attachments (Cheeseman et al., 2006). Ndc80 complex function will be discussed in greater detail in Chapters 3-5. Zwint, a vertebrate protein that localizes to the kinetochore throughout mitosis and interacts with KNL-1/Blinkin (Emanuele et al., 2005; Kiyomitsu et al., 2010; Petrovic et al., 2010), could be included as the tenth member of the vertebrate KMN complex. The outer kinetochore also contains CENP-F, a 367-kDa protein that recruits dynein complexes to kinetochores and facilitates proper kinetochore-microtubule attachments (Feng et al., 2006; Vergnolle and Taylor, 2007).

\section{The fibrous corona}

The fibrous corona represents the most centromere-distal region of the kinetochore and is lost from the kinetochore following microtubule attachment (McEwen et al., 1998). Many of the proteins comprising this region are thought to be stripped via the poleward movement of the minus-end tracking motor protein dynein (Howell et al., 
2001). The amount of fibrous corona proteins present at the kinetochore is exquisitely sensitive to microtubule attachment status. In addition to being rapidly lost following microtubule attachment, an excess of corona proteins can be driven to the kinetochore by treating cells with nocodazole, which removes nearly all microtubules from the cell (Hoffman et al., 2001). Fibrous corona proteins can be divided into two classes. The first class includes factors involved in capturing microtubules and forming initial attachments to kinetochores. These initial attachments are transient connections and are referred to as lateral attachments. Lateral attachments are mediated by proteins such as dynein and CENP-E, and are not stable enough to facilitate depolymerization-coupled movement (Kapoor et al., 2006; Vorozhko et al., 2008). Therefore, lateral attachments must be converted to more stable bi-polar attachments in which microtubules embed end-on into the outer kinetochore plate. This poorly understood conversion process is referred to as attachment maturation.

A second group of mitotic checkpoint proteins resides in the corona region to monitor microtubule attachment status. This group includes Mad1, Mad2, Bub1, Bub3, BubR1 (Mad3), and MPS1 (Hoyt et al., 1991; Li and Murray, 1991; Weiss and Winey, 1996). Vertebrate kinetochores also contain three additional checkpoint proteins - Rod, Zwilch and ZW10 (Chan et al., 2000; Williams et al., 2003). These three proteins comprise the RZZ complex, which (along with the newly identified Spindly protein) serve to regulate both checkpoint signaling and the timing of attachment maturation (Barisic et al., 2010; Gassmann et al., 2008; Karess, 2005). 


\section{Properties of kinetochores}

\section{Kinetochores exist in different sizes}

All kinetochores are assembled on centromeric chromatin, but assembly is achieved in distinct ways across species. Organisms such as budding yeast (Saccharomyces cerevisiae) assemble kinetochores on point centromeres. S. cerevisiae kinetochores are assembled on a 125-bp stretch of DNA. In contrast, fission yeast (Saccharomyces pombe) and metazoan kinetochores are assembled on regional centromeres. Human kinetochores are assembled on centromeric DNA that is more than $10 \mathrm{Mb}$ in length and contains numerous $\alpha$-satellite repeats (Cleveland et al., 2003). Caenorhabditis elegans kinetochores are assembled on regional centromeres but their kinetochores are holocentric, or spread across the length of the chromosome arms.

There also is variability in the number of microtubules a kinetochore binds. Budding yeast kinetochores bind a single microtubule (Peterson and Ris, 1976) while fission yeast kinetochores bind 2-4 (Ding et al., 1993). By contrast, human kinetochores bind approximately 15-30 microtubules (collectively referred to as a K-fiber) (Rieder, 1982). Therefore, kinetochores must be built to meet species-specific requirements for generating and regulating microtubule attachments.

\section{Kinetochore proteins have evolved}

While many of the most well studied kinetochore proteins (such as Ndc80, KNL1 and CENP-A) are conserved across species, the sequences of these proteins have often evolved quite rapidly. A 2006 study by Patrick Meraldi and Andrew McAinsh concluded that while many proteins used to assemble the kinetochore retain their structural domains throughout evolution, other parts of these proteins (as well as other kinetochore proteins) 
have rapidly evolved (Meraldi et al., 2006). At times, this sequence evolution translates into functional divergence of a kinetochore protein (Guimaraes et al., 2008; Kemmler et al., 2009; Miller et al., 2008; Rahmani et al., 2009). The authors speculate that this divergence might have arisen to meet species-specific needs.

Many kinetochore proteins that exist in metazoans are absent in S. cerevisiae, and vice versa. The RZZ complex, the Ska complex, HURP, CENP-E and CENP-F are some of the vertebrate kinetochore proteins not found in budding yeast. Conversely, budding yeast kinetochores contain Dam1, CBF3 and (some) $\mathrm{Ctf} 19$ complex proteins which are absent in vertebrates (Santaguida and Musacchio, 2009). Discrepancies also exist between metazoan species. At D. melanogaster and C. elegans kinetochores, some members of the CCAN have not been identified. However, this structural complex is essential for both vertebrate and yeast kinetochore function (Cheeseman and Desai, 2008). So in addition to affecting individual protein function, these species-specific differences can also alter kinetochore assembly.

\section{Kinetochore assembly}

The conserved histone H3 variant CENP-A marks the site of kinetochore assembly and is critical for proper kinetochore function (Howman et al., 2000). In vertebrates, CENP-A is in turn responsible for recruiting the Constitutive Centromere Associated Network (CCAN) to the kinetochore (Foltz et al., 2006; Howman et al., 2000; Izuta et al., 2006; Okada et al., 2006). Members of the CCAN serve as the platform for assembly of more centromere-distal layers of the kinetochore. One of the best-studied members of the CCAN is CENP-C, a protein that directly interacts with CENP-A 
containing chromatin. The N-terminus of CENP-C directly binds to the Nnf-1 subunit of the Mis12 complex in both humans and D. melanogaster, providing a direct link between the inner and outer kinetochore layers (Przewloka et al., 2011; Screpanti et al., 2011). Within the human Mis12 complex, a second subunit (Nsl1) directly contacts both KNL1/Blinkin and the C-terminal region of the Ndc80 complex - thereby allowing kinetochore recruitment of the KMN super-complex (Petrovic et al., 2010). The KMN network in turn serves as an outer kinetochore platform that recruits additional microtubule binding and spindle checkpoint factors (Musacchio and Salmon, 2007). As these proteins are conserved across species, the CENP-A to CENP-C to KMN assembly pathway can be considered a general assembly framework for kinetochores (Cheeseman and Desai, 2008). However, extensive studies of protein sequence, assembly dependencies and physical interactions have demonstrated that variations on this theme exist throughout evolution (Cheeseman et al., 2006; Cheeseman et al., 2008; Cheeseman et al., 2004; Meraldi et al., 2006; Petrovic et al., 2010; Schueler et al., 2010).

Despite slightly divergent assembly pathways, kinetochores have largely maintained the same number of KMN proteins present at each microtubule attachment site. Immunofluorescence studies from the Salmon laboratory have demonstrated that budding yeast, fission yeast and chicken (Gallus gallus) kinetochores all largely contain the same number of KMN proteins at each microtubule attachment site - despite being responsible for binding a wide range of microtubules (Joglekar et al., 2008; Joglekar et al., 2006; Johnston et al., 2010). These data argue for a repeat subunit model for kinetochores, which postulates that kinetochores have scaled up from the single microtubule binding "subunit" employed by budding yeast (Zinkowski et al., 1991). One 
notable exception to this rule is observed with the yeast Dam1 protein. In budding yeast, where this protein is essential, there are 16 Dam1 molecules per microtubule. In fission yeast - where Dam1 aids in microtubule attachment but is not essential - there is one Dam1 molecule per MT (Joglekar et al., 2008).

\section{The dynamics of kinetochore-microtubule interactions}

A dynamic series of changes are set into motion as kinetochores come into contact with microtubules. These changes are bi-directional - kinetochores perform work on bound microtubules, and microtubules in turn trigger a series of changes to kinetochore composition. The mechanistic nature of kinetochore-microtubule interactions remains a subject of much debate. A number of models exist to describe attachment formation, but central to each model is the recognition that the interface between kinetochores and microtubules constitutes a highly dynamic micro-environment - full of attachment points, conformational changes and signaling modules all working together to ensure faithful chromosome segregation.

\section{Kinetochores perform work on microtubules}

It has long been recognized that the dynamics of a kinetochore-bound microtubule differ from an unbound microtubule (both in vitro and in vivo). Brinkley and Cartwright first observed that when mitotic cells were treated with cold temperatures, chromosomal microtubules were refractory to this treatment while inter-polar and astral microtubules were rapidly depolymerized (Brinkley and Cartwright, 1975). Borisy and colleagues later demonstrated that the turnover rate for kinetochore-microtubules (kMTs) in metaphase was twice as slow as non-kinetochore bound microtubules (Zhai et al., 1995). More 
recently, McEwen and colleagues demonstrated that while K-fibers elongate and shorten in a coordinated manner, individual microtubules within the $\mathrm{K}$-fiber are both growing and shrinking (VandenBeldt et al., 2006). These studies and others point to a highly complex regulatory environment at the kinetochore.

With the discovery of individual kinetochore proteins, we now can assign subsets of proteins to specific microtubule functions. Proteins such as MCAK and Kif2a cause microtubules to depolymerize by triggering catastrophe (Desai et al., 1999b; Kline-Smith and Walczak, 2002; Knowlton et al., 2009), while other proteins such as EB1, CLASP and chTOG/XMAP215 promote the polymerization and stability of kinetochore microtubules (Al-Bassam et al., 2010; Brouhard et al., 2008; Hayashi et al., 2005). Additionally, proteins such as Hec1/Ndc80 and Dam1 allow kinetochores to couple to a microtubule and hence prevent detachment. Therefore, while kinetochore-bound microtubules remain dynamic, those dynamics are altered following interaction with the regulatory machinery of the kinetochore.

\section{Microtubules perform work on kinetochores}

Following contact with a microtubule, the structural composition of a kinetochore is also greatly altered. There are two reasons for this. First, the presence of a microtubule provides a conduit for the minus-end tracking motor protein dynein. Dynein levels are greatly elevated at unattached kinetochores but quickly drop following microtubule attachment. Dynein is thought to transport a number of transient attachment and checkpoint factors from kinetochores to spindle poles - a process referred to as "stripping" and likely the cause of the disappearance of the fibrous corona following attachment (Howell et al., 2001). Proteins removed from the kinetochore by dynein 
include the RZZ complex, Mad2, Spindly and CENP-E (Famulski et al., 2011; Gassmann et al., 2010; Howell et al., 2001; Wojcik et al., 2001).

Recent work has illuminated a second consequence of microtubule attachment by the kinetochore. Many of the stable kinetochore components (those that remain through anaphase and are not stripped away by dynein) are instead stretched relative to one another following microtubule attachment. This molecular re-arrangement is thought to be due to the pulling forces exerted on the kinetochore by dynamic microtubules. Salmon and colleagues first demonstrated this re-arrangement using a high-resolution imaging technique called K-SHREC (Wan et al., 2009). Uchida and Maresca later published a pair of studies correlating these intra-kinetochore stretching forces to checkpoint signaling status (Maresca and Salmon, 2009; Uchida et al., 2009). (This subject will be further discussed in Chapter 3). These changes in kinetochore conformation and the way they integrate with spindle checkpoint signaling represent an exciting area of future research.

\section{Models for kinetochore-microtubule attachment}

A kinetochore must bind microtubules strongly enough to move a chromosome through the cell. However, kinetochore-bound microtubules remain dynamic - both adding and subtracting subunits from their plus ends (Hunt and McIntosh, 1998). This presents a paradoxical question, one that has spurned numerous models for kinetochoremicrotubule attachment. As dynein and the kinesin-like CENP-E were two of the first kinetochore proteins identified, early models posited that motor proteins controlled chromosome movement by harnessing the power stroke generated by ATP hydrolysis (Pfarr et al., 1990; Rosenfeld et al., 2009; Shima et al., 2006; Yen et al., 1992). However, in 1988 Doug Koshland demonstrated that it was microtubule depolymerization that 
drove chromosome movements in vitro. These depolymerization-coupled movements occurred in the absence of ATP, arguing that kinetochores did not require motor protein activity to facilitate chromosome movement (Koshland et al., 1988). While motor proteins do enhance the fidelity of chromosome alignment, they are dispensable for anaphase movements in vivo (Howell et al., 2001; Vorozhko et al., 2008). Therefore, kinetochores must use an alternate mechanism to couple microtubule dynamics to chromosome movement. In recent years the most prominent theories put forth have been the Hill Sleeve and Sliding Ring models. EM-based models invoking kinetochore meshworks or fibrils have also been proposed. These models remain the subject of much debate, and importantly need not be mutually exclusive or used to the same extent throughout evolution.

\section{The Hill Sleeve Model}

In 1985 Terrell Hill put forth a mathematical model to explain the observed properties of kinetochores and microtubules (Hill, 1985). Hill envisioned a kinetochore sleeve containing numerous low-affinity attachment points capable of binding along the side of a microtubule. Individual attachments would be weak enough that they could easily be broken and re-formed, allowing the kinetochore to diffuse along the lateral surface of the tubulin polymer. However, the overall energy requirements of breaking all these interactions would be prohibitively high - allowing the kinetochore to maintain attachment during periods of microtubule growth or shrinkage. Importantly, by binding along the side of the microtubule the sleeve would allow for gain or loss of tubulin subunits from the plus end of the microtubule. During anaphase, when microtubules 
rapidly depolymerize, tubulin attachment points would be rapidly lost from the plus end of the microtubule - biasing the kinetochore to move toward the spindle pole. This type of movement was therefore named "biased diffusion". A number of kinetochore proteins have been shown to bind microtubules with low affinity and to track depolymerizing microtubules in vitro - lending credence to this model for kinetochore function (Cheeseman et al., 2006; Ciferri et al., 2008; Welburn et al., 2009).

\section{The Sliding Ring Model}

The Sliding Ring Model envisions the kinetochore forming a ring-like structure that would encircle a captured microtubule. In this model, the ring would topologically grip the microtubule (preventing detachment) while allowing the kinetochore to slide forward and backward along the surface of the dynamic polymer. During periods of microtubule depolymerization, this model envisions the kinetochore moving toward the spindle pole in anaphase by a "forced walk" mechanism - with the kinetochore coupler being pushed toward the microtubule minus end by the conformational splaying occurring at the plus end (Davis and Wordeman, 2007; Joglekar et al., 2010). In recent years work on the budding yeast Dam1 complex has generated much excitement over this model. In vitro work has demonstrated that members of the Dam1 complex possess many attributes of a ring-based coupler that are posited by this model (Asbury et al., 2006; Grishchuk et al., 2008a; Miranda et al., 2005; Nogales and Ramey, 2009; Westermann et al., 2005; Westermann et al., 2006). However, the Dam1 complex is not conserved outside of yeast and is only essential in budding yeast. Therefore, it remains an open question whether vertebrate kinetochores utilize a similar mechanism to couple to 
microtubules. As Dam1 patches also are capable of serving as kinetochore couplers (Gao et al., 2010; Gestaut et al., 2008; Grishchuk et al., 2008b), it is also possible that a ringlike structure would not be required to generate this type of depolymerization-coupled movement.

\section{Meshworks and fibrils}

Recent advances in EM technology have allowed for greater resolution of kinetochore structure following microtubule attachment. In 2007, McEwen and colleagues proposed a meshwork model for the kinetochore. Briefly, based on EM data the authors proposed that unattached kinetochore proteins exist as a fibrous meshwork (Dong et al., 2007). Following microtubule attachment, this meshwork becomes rearranged into a structure with two distinct microtubule attachment points. The molecular re-arrangements observed by Salmon lab using K-SHREC further support this model for attachment (Wan et al., 2009). In 2008, McIntosh and colleagues used EM of Ptk1 kinetochores to observe kinetochore fibrils binding along the insides of depolymerizing microtubules (McIntosh et al., 2008). These fibrils were seen contacting microtubules near the tip and restraining the pronounced splaying normally seen as a microtubule depolymerizes. Based on these data, the authors proposed a coupling mechanism whereby kinetochore fibrils attached to the luminal side of a depolymerizing microtubule would constrain the splaying triggered by depolymerization (and hence stabilize kinetochore-microtubule attachments) but would also harness the residual energy generated by protofilament curvature to drive chromosome movement. Such a mechanism would also eliminate the need for a ring in vertebrate kinetochores (Bloom, 
2008). One critical caveat of both EM-derived models is the inability of this technique to assign a molecular identity to any of the shapes observed. While both the meshwork and the fibrils are presumed to represent microtubule-binding factors such as $\mathrm{Hec} 1 / \mathrm{Ndc} 80$, KNL-1 and CENP-F, this element of both models remains unproven. 
Figure 1-3: Models for kinetochore-microtubule coupling

(A) The Hill sleeve model envisions a kinetochore sleeve containing numerous lowaffinity attachment points capable of binding along the side of a microtubule and allowing the kinetochore to undergo biased diffusion during periods of microtubule depolymerization. (B) The fibrillar coupling model envisions kinetochore fibrils attached to the luminal side of a depolymerizing microtubule that would constrain the splaying triggered by depolymerization (and hence stabilize kinetochore-microtubule attachments) but would also harness the residual energy generated by protofilament curvature to drive chromosome movement. (C) The Sliding Ring model envisions a kinetochore-localized ring that would topologically grip a microtubule (preventing detachment) while allowing the kinetochore to slide forward and backward along the microtubule surface. During periods of microtubule depolymerization, this model envisions the kinetochore moving toward the spindle pole in anaphase by a "forced walk" mechanism - with the ring coupler being pushed toward the microtubule minus end by the conformational splaying occurring at the microtubule plus end. 
(A)

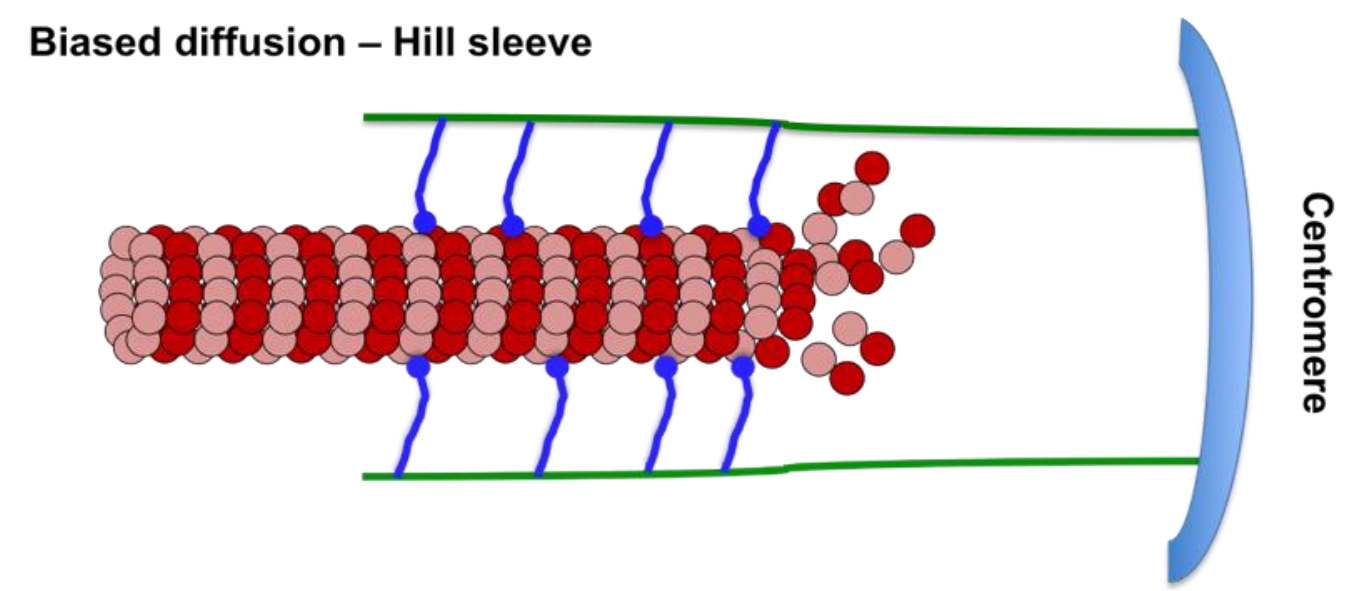

(B)

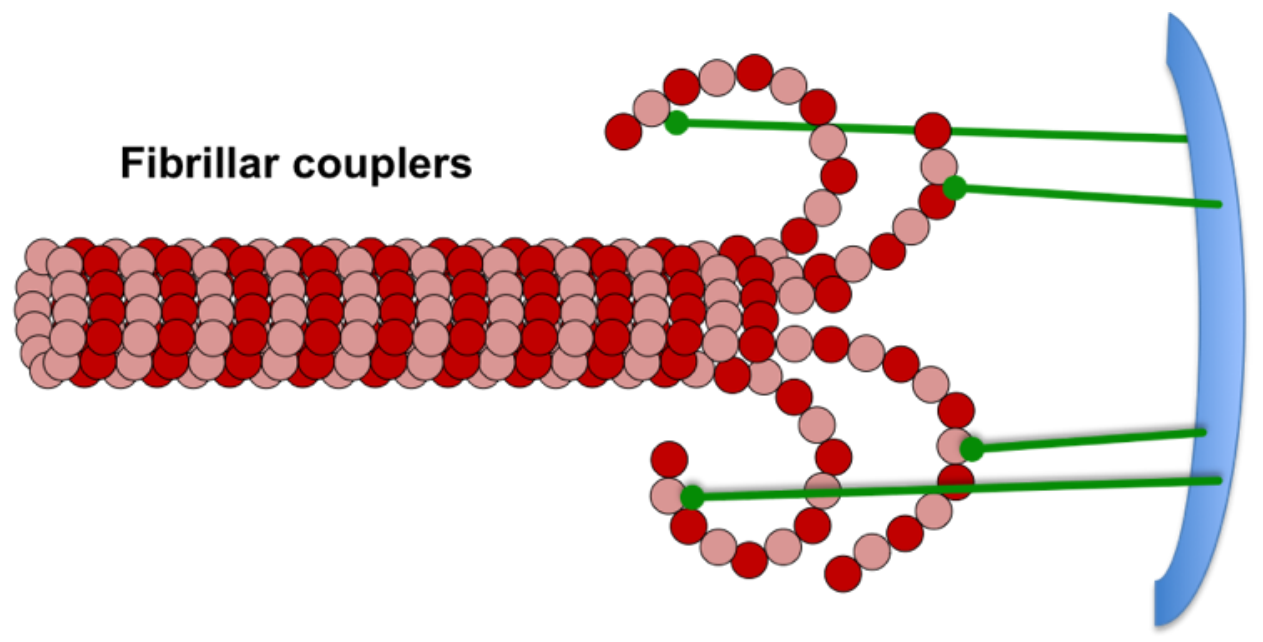

(C)

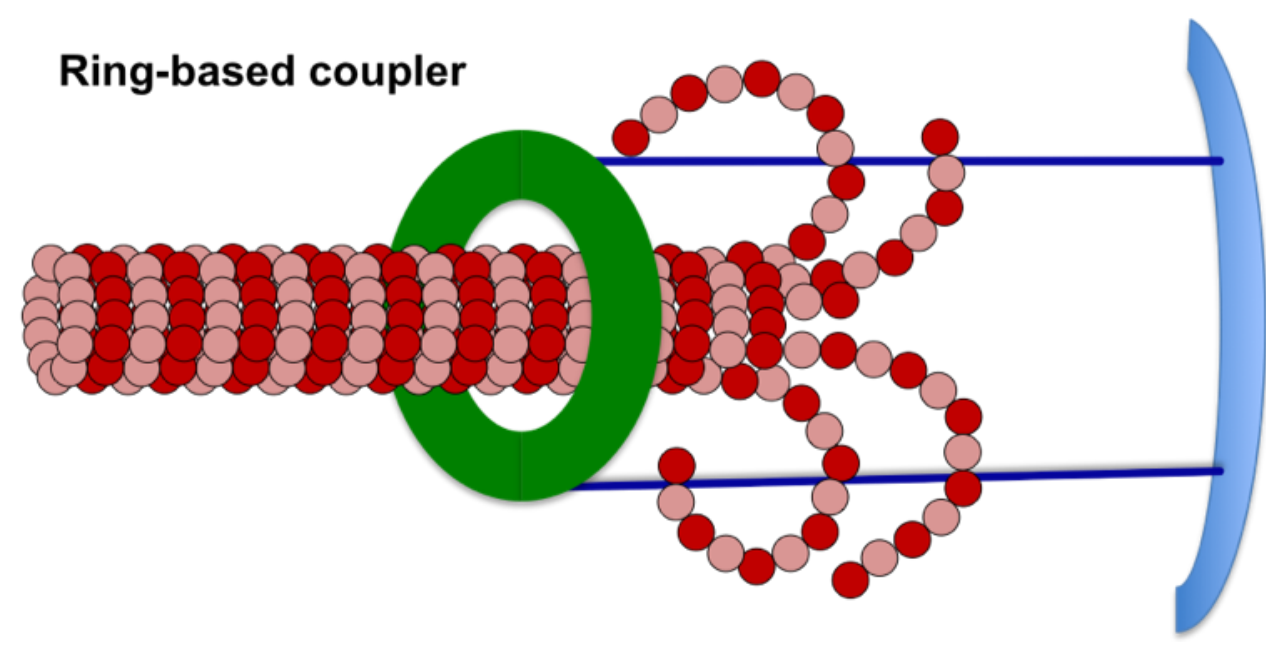

Figure 1-3 


\section{Preventing improper mitosis - and the consequences of failure}

\section{Detecting and repairing improper kinetochore-microtubule attachments}

Proper chromosome segregation requires kinetochores to form bi-polar, amphitelic attachments to spindle microtubules. In this binding geometry, sister kinetochores are bound exclusively to microtubules emanating from opposite spindle poles. However, microtubule capture is a stochastic procedure and errors often occur (Cimini et al., 2003). Improper attachments include syntelic attachments, where both sister kinetochores bind microtubules from a single pole, and merotelic attachments, where a single sister kinetochore binds microtubules from both poles. Failure to correct these attachment errors can lead to improper chromosome segregation (Cimini et al., 2001). To safeguard this process, the kinetochore monitors its attachment status and utilizes mechanisms to both halt cell cycle progression and to correct attachment errors. The Spindle Assembly Checkpoint (SAC) is a cell cycle checkpoint that blocks cell cycle progression (more specifically the metaphase-to-anaphase transition) in response to the presence of even a single unattached kinetochore (Burke and Stukenberg, 2008; Musacchio and Salmon, 2007). The SAC functions by restraining the activity of the Anaphase Promoting Complex/Cyclosome (APC/C), an E3-ubiquitin ligase that drives a cell out of mitosis by degrading numerous mitotic proteins, including securin and cyclin B1.

In the presence of an unattached or improperly attached kinetochore, a checkpoint signal is sent which inactivates APC/C and allows the cell additional time to correct attachment errors. The Aurora B kinase is one of the key proteins involved in correcting improper attachments (Biggins and Murray, 2001; Hauf et al., 2003; Tanaka et al., 2002). 
By phosphorylating a number of microtubule-binding proteins, Aurora B has been shown to reduce the ability of its targets to bind microtubules. The detection and reversal of erroneous kinetochore-microtubule interactions represents a key step in ensuring the fidelity of chromosome segregation, but the precise mechanism by which this is achieved remains quite controversial (Khodjakov and Pines, 2010; Khodjakov and Rieder, 2009; Maresca and Salmon, 2010; Santaguida and Musacchio, 2009; Santaguida et al., 2011).

\section{Kinetochores and cancer}

Failure to properly segregate chromosomes leads to chromosome instability (CIN), which causes aneuploidy, a hallmark of many types of cancers. Indeed, numerous kinetochores protein have been implicated in cancer - including Aurora A, TPX2, Aurora B, Survivin, Hec1/Ndc80, CENP-H, Bub1, and BubR1 (Asteriti et al., 2011; DiazRodriguez et al., 2008; Grabsch et al., 2003; Nguyen and Ravid, 2006; Tomonaga et al., 2005). Misregulation of these proteins has generated interest in studying kinetochore proteins as possible cancer targets. Because many of these proteins are only required during mitosis, their disruption would theoretically be less toxic to patients than tubulin poisons such as taxanes or vinca alkyloids, which also disrupt interphase cellular processes. However, it remains uncertain whether or not CIN due to alteration of the kinetochore can cause cancer in humans (Holland and Cleveland, 2009; Thompson and Compton, 2008). Kinetochore proteins also have not been successfully targeted as treatments for cancer. One reason could be the limited efficacy these treatments would be hypothesized to have on slow-growing tumors where the majority of the cell population is senescent. 


\section{ChAPTER 2: StruCture-FunCtion AND PHENOTyPIC ANALYSIS OF THE CENTROSOME PROTEINS CEP57R AND CEP57}

This chapter is based on unpublished work. 


\section{Abstract}

In anaphase, kinetochores stably bind spindle microtubules to facilitate depolymerization-coupled movement (Koshland et al., 1988). Additionally, spindle poles must anchor the minus ends of spindle microtubules in order to exert the opposing forces required for directional movement of chromosomes (Gordon et al., 2001). The mechanisms of how kinetochores and spindle poles bind microtubules remain critical unanswered questions.

One proposed model for kinetochore-microtubule interactions invokes a sliding ring coupler in which a kinetochore-bound ring attaches to spindle microtubules. However, strong evidence for such a model only exists in budding yeast (Nogales and Ramey, 2009). To ask whether vertebrate kinetochores employ a similar ring coupling mechanism, our lab attempted to identify a functional vertebrate homolog of the $S$. cerevisiae ring protein Dam1. We characterized a novel mitotic regulator (now named Cep57R) that co-localizes with both kinetochores and spindle poles on spindles assembled in Xenopus laevis egg extracts. Both kinetochore and centrosome-specific phenotypes were observed following immunodepletion or functional inhibition of $\mathrm{xCep57R}$ using a polyclonal antibody generated against this protein. This places $\mathrm{xCep57R}$ in the unique position of potentially regulating microtubule attachment at both ends of the mitotic spindle (Emanuele and Stukenberg, 2007). However, little else is known about the mechanism of $\mathrm{xCep57R}$ function and whether this function is conserved in humans.

Cep57R also has a closely related family member, Cep57. Both proteins bind microtubules, localize to centrosomes, and are phosphorylated by Aurora kinases. 
Interestingly human Cep57 and Cep57R physically interact, though the presence of one is not required for the stability of the other. Knockdown of either protein in HeLa cells does not generate robust phenotypes, and neither protein can be detected at human kinetochores. I discuss possible reasons for the discrepancies between human and Xenopus laevis Cep57R and propose future experiments to explore a role for Cep57R and Cep57 in mitosis. 


\section{Background}

The focus of our lab has been on Cep57R due to its sequence similarity to Dam1, but Cep57 is the better studied of the two proteins. Also known as Translokin or TestisSpecific Protein 57 (Tsp57), Cep57 (Centrosomal protein 57-kDa) was first discovered in 2003 as both a mediator of FGF-2 trafficking to the nucleus (Bossard et al., 2003) and as a salt-insensitive component of the human centrosome (Andersen et al., 2003). While the protein is expressed in most tissue types (Bossard et al., 2003), Cep57 is most highly expressed in the developing testis (Kim et al., 2004).

During interphase, Cep57 co-localizes with the microtubule network and MTOCs. Overexpression of Cep57 in cells causes the formation of thick microtubule cables that are resistant to nocodazole treatment (Momotani et al., 2008). Knockdown of Cep57 results in defects in the nuclear accumulation of both FGF-2 and Cyclin D1 during interphase, although effects on overall cell viability are minimal (Bossard et al., 2003; Meunier et al., 2009; Ruiz-Miro et al., 2011). Cep57 interacts with four proteins implicated in the bi-directional trafficking of FGF-2 during interphase. Sorting nexin 6 (SNX6) and Ran-Binding Protein M (RanBPM) are involved in internalization and retrograde transport of proteins, while KIF3A and KIF3B are involved in anterograde transport of proteins along microtubules. A recent study identified an additional 24 proteins as hCep57-interactors but their identities were not revealed (Meunier et al., 2009).

In mitosis, Cep57 localizes to microtubules and spindle poles. Knockdown of mCep57 protein results in reduced pole-to-pole distance of mitotic spindles but has no appreciable affect on cell viability. Overexpression of mCep57, by contrast, ultimately 
leads to cell death - presumably due to the thick cables of microtubules generated by this treatment (Momotani et al., 2008). Mutations in human Cep57 were recently identified in patients with mosaic variegated aneuploidy syndrome (MVA), a rare autosomal recessive disorder. Another subpopulation of MVA patients harbored mutations in the spindle checkpoint kinase Bub1 (Snape et al., 2011). Structurally, Cep57 is characterized by two coiled-coil domains separated by three small regions of unstructured sequence. The Nterminal domain of Cep57 is required for protein multimerization and centrosomal localization, while the C-terminal domain of Cep57 is required for interaction with microtubules (Momotani et al., 2008). The amino acid sequence of Cep57 (500 amino acids long in humans) is well conserved across species, especially in the two predicted coiled-coil regions (Figure 2-1B).

A second Cep57 family member exists in vertebrates. Like Cep57, Cep57R is 500 amino acids long and contains two predicted coiled-coil domains separated by three small regions of unstructured sequence (Figure 2-1A). At the amino acid level, there is $30 \%$ sequence similarity between Cep57 and Cep57R (Figures 2-1D and 2-1E), with the majority of the conservation again occurring in the two predicted coiled-coil regions. Interestingly, Cep57R localizes to microtubules and spindle poles (Emanuele and Stukenberg, 2007). A polyclonal antibody generated against the full-length xCep57R protein also localizes to kinetochores assembled on sperm chromatin (in Xenopus egg extracts) and on chromosomes isolated from mitotically arrested Xenopus tissue culture cells (Emanuele and Stukenberg, 2007). 
Figure 2-1: Domains of Cep57R and sequence alignments for Cep57R and Cep57

(A) Cep57R is characterized by two predicted coiled-coil domains separated by three smaller regions of unstructured sequence. In Xenopus laevis, Cep57R is 488 amino acids long. (B) Alignment of mouse, rat, human and Xenopus laevis Cep57 amino acid sequences. The greatest amino acid sequence conservation occurs in the two predicted coiled-coil domains. Green coded residues are conserved across all species examined; yellow coded residues are conserved across most of the species examined. Blue coded residues share common properties (hydrophobic, acidic, etc.) (C) Alignment of mouse, human, rat, Xenopus tropicalis, and Xenopus laevis Cep57R amino acid sequences. The greatest amino acid sequence conservation occurs in the two predicted coiled-coil domains. (D) Amino acid sequence alignment of Xenopus laevis Cep57 and Cep57R. There is $\sim 32 \%$ conservation between the two proteins, mostly in the coiled-coil regions. (E) Amino acid sequence alignment of human Cep57 and Cep57R. There is $\sim 30 \%$ conservation between the two proteins, mostly in the coiled-coil regions. 
A

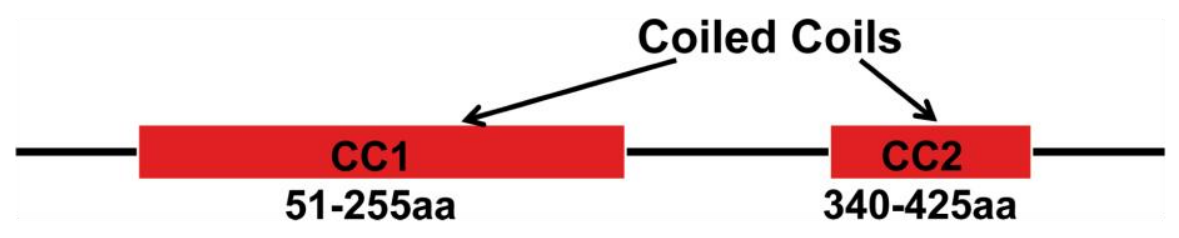

xCep57R

B

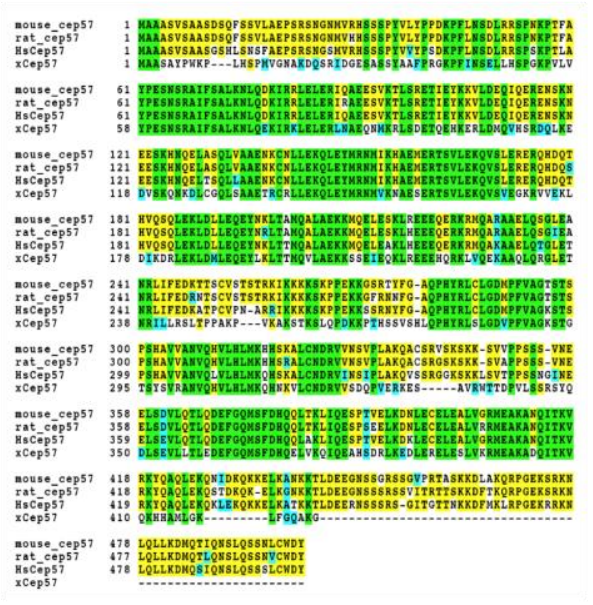

C

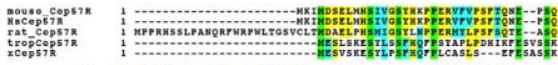

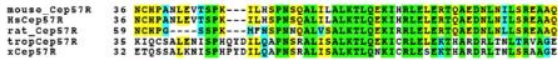

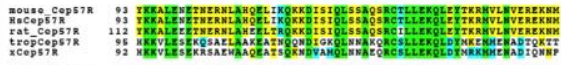

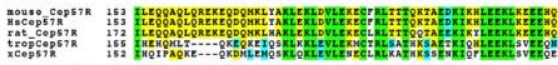

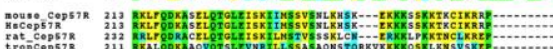

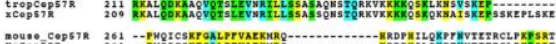

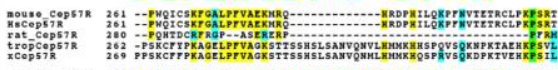

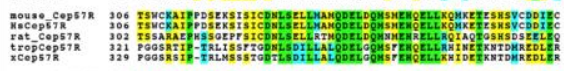

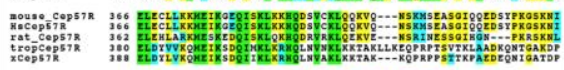

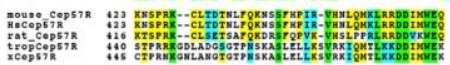

D

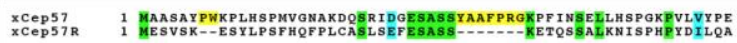

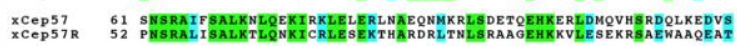

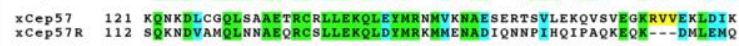

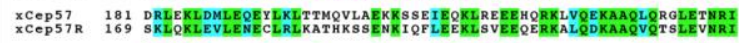

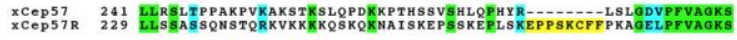

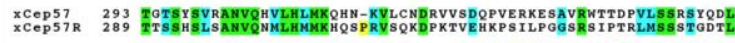

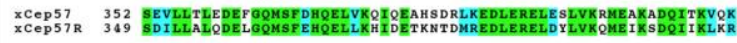

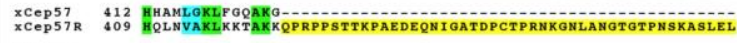

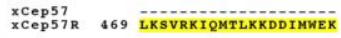

E

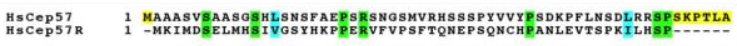

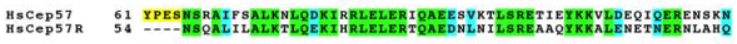

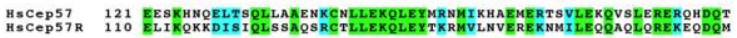
HSCCP 57
HSCP

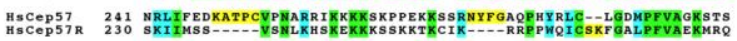

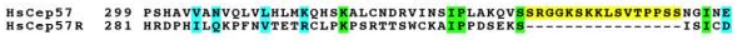

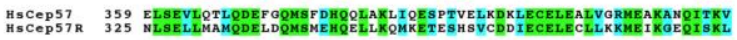

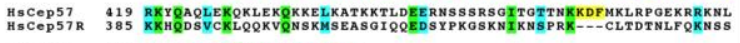
Hscep57
HsCoP57R

Figure 2-1 
Our lab became interested in $\mathrm{xCep} 57 \mathrm{R}$ because this protein displayed weak sequence similarity to the $\mathrm{N}$-terminal 90 amino acids of the budding yeast Dam1 protein. In budding yeast Dam1 is one of 10 proteins required to form the Dam1 ring complex, which can bind and processively track along depolymerizing microtubules (discussed in Chapter 1). Therefore, we asked if $\mathrm{xCep57R}$ acted as a functional vertebrate homolog to Dam1. Immunodepletion of $\mathrm{xCep57R}$ from mitotic Xenopus egg extracts generated strong phenotypes, including elongated bipolar spindles, an inability to congress chromosomes into a metaphase plate, and a diminished incorporation of microtubules into the mitotic spindle. Addition of antibodies against xCep57R blocked kinetochoremicrotubule interactions in vitro, and also impaired the anchorage of microtubules nucleated from purified centrosomes (Emanuele and Stukenberg, 2007). These data suggest that $\mathrm{xCep57R}$ functions at both ends of the mitotic spindle.

Imunnoprecipitations of xCep57R from mitotic Xenopus egg extracts identified interactions with the kinetochore proteins Mis12, Zwint, and CLIP-170. Interestingly, mCep57 protein is also able to pull down numerous kinetochore and centrosome proteins from Xenopus egg extracts. Cep57-interacting proteins include all members of the HURP complex, a five-protein complex implicated in facilitating spindle bipolarity in Xenopus egg extracts (Koffa et al., 2006). Notably, this complex contains proteins that localize to the kinetochore and others that localize to spindle poles. We hypothesized that Cep57, like Cep57R, might function at both ends of the mitotic spindle.

Based on these data, we set out to better understand the mechanism of Cep57R and Cep57 function. We also asked whether a direct interaction exists between Cep57R and Cep57, and whether Cep57R function was conserved from Xenopus to humans. 


\section{Results}

\section{$x$ Cep57R directly binds microtubules}

xCep57R co-sediments with taxol-stabilized MTs spun out of Xenopus egg extracts (Emanuele and Stukenberg, 2007), but whether the binding reaction is direct or indirect remains unclear. To ask if $\mathrm{xCep57R}$ directly binds MTs, we generated recombinant full-length $\mathrm{xCep57R}$ protein tagged at its N-terminus with GST. We also generated a series of GST-tagged fragments of the xCep57R protein and a GST-only control (Figure 2-2A). Proteins were incubated with purified taxol-stabilized microtubules for 10 minutes at room temperature and then spun through a glycerol cushion. Proteins were recovered from the supernatant and pellet fractions and subjected to SDS-PAGE gel electrophoresis followed by Coomassie staining. No proteins sediment in the absence of microtubules, arguing that these proteins are not aggregating. At $5 \mu \mathrm{M}$ tubulin subunit concentration, full-length $\mathrm{xCep57R}$ efficiently pellets with microtubules while the control GST-only protein does not. The extreme $\mathrm{N}$ - and C-terminal

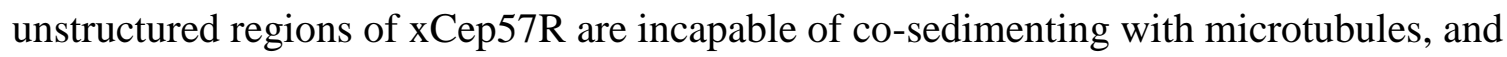
both the $\mathrm{N}$ - and $\mathrm{C}$-terminal halves of the protein bind microtubules poorly. This suggests that either multiple regions of $\mathrm{xCep57R}$ are required for coordinating MT binding or that xCep57R must dimerize to efficiently bind MTs. 
Figure 2-2: Full-length $x$ Cep57R directly binds microtubules

(A) Cartoon schematic of recombinant N-terminally GST-tagged xCep57R proteins used for microtubule binding studies. (B) Full length xCep57R co-sediments with microtubules, but individual domains of the protein cannot efficiently bind microtubules. $150 \mathrm{nM}$ of recombinant $\mathrm{xCep57R}$ protein was incubated with the indicated amounts of taxol-stabilized microtubules (concentration represents tubulin monomer) for 10 minutes at room temperature. The reaction mixture was sedimented through a glycerol cushion and both supernatant (Supe) and the pellet (Pellet) samples were collected. Samples were subjected to SDS-PAGE and proteins were identified by Western blotting for GST. 
A

$$
\text { }
$$
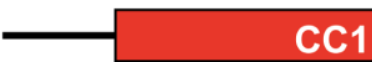

$\mathrm{CC} 2$

FL: 1-488 aa
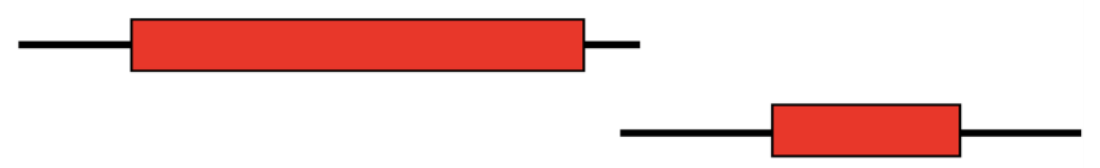

NH: 1-280 aa

CH: $280-488$ aa

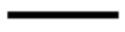

NT: 1-50 aa

- CT: $425-488$ aa

B

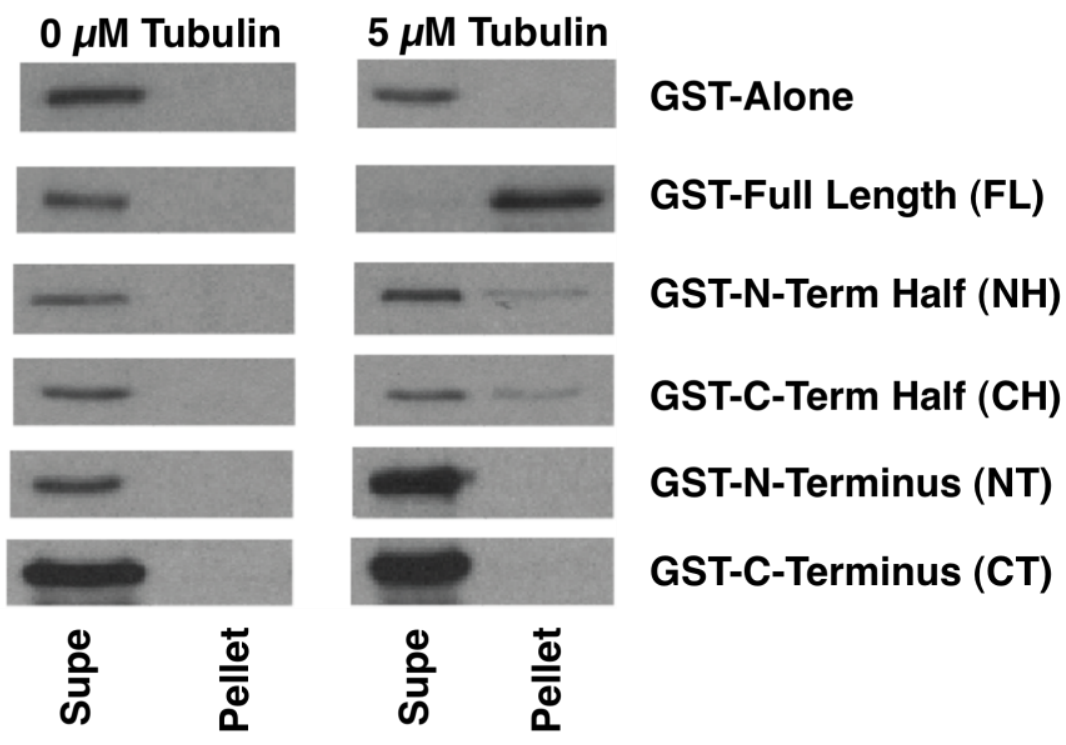

Figure 2-2 
$x$ Cep57R does not form rings around microtubules

As the recombinant Dam1 complex forms rings around microtubules in vitro (Westermann et al., 2005), we next used EM staining to ask what structures xCep57R formed when bound to microtubules. We incubated $150 \mathrm{nM}$ xCep57R protein with $5 \mu \mathrm{M}$ taxol-stabilized microtubules for 10 minutes at room temperature before placing samples on EM grids. Uranyl acetate stain was added to the sample and grids were further processed for visualization. $\mathrm{xCep57R}$ was seen oligomerizing and occasionally contacting microtubules (Figure 2-3). While a variety of shapes were observed in our EM

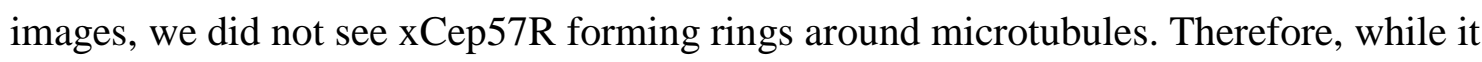
remains possible that $\mathrm{xCep57R}$ is one part of a multi-protein complex capable of forming a ring, we conclude that $\mathrm{xCep57R}$ on its own cannot form rings around a microtubule. 
Figure 2-3: EM images of $x$ Cep57R bound to microtubules

(A) Negative-stained EM image of $5 \mu \mathrm{M}$ taxol-stabilized microtubules. No recombinant $\mathrm{xCep57R} \mathrm{protein} \mathrm{is} \mathrm{present.} \mathrm{Inset} \mathrm{shown} \mathrm{below} \mathrm{is} \mathrm{delineated} \mathrm{by} \mathrm{box} \mathrm{in} \mathrm{top} \mathrm{image.} \mathrm{Scale}$ bar $=100 \mathrm{~nm}$. (B) Negative-stained EM image of $5 \mu \mathrm{M}$ taxol-stabilized microtubules that have been incubated with $150 \mathrm{nM}$ recombinant $\mathrm{xCep} 57 \mathrm{R}$ protein for 10 minutes at room temperature. Inset shown below is delineated by box in top image. Scale bar $=100 \mathrm{~nm}$. 

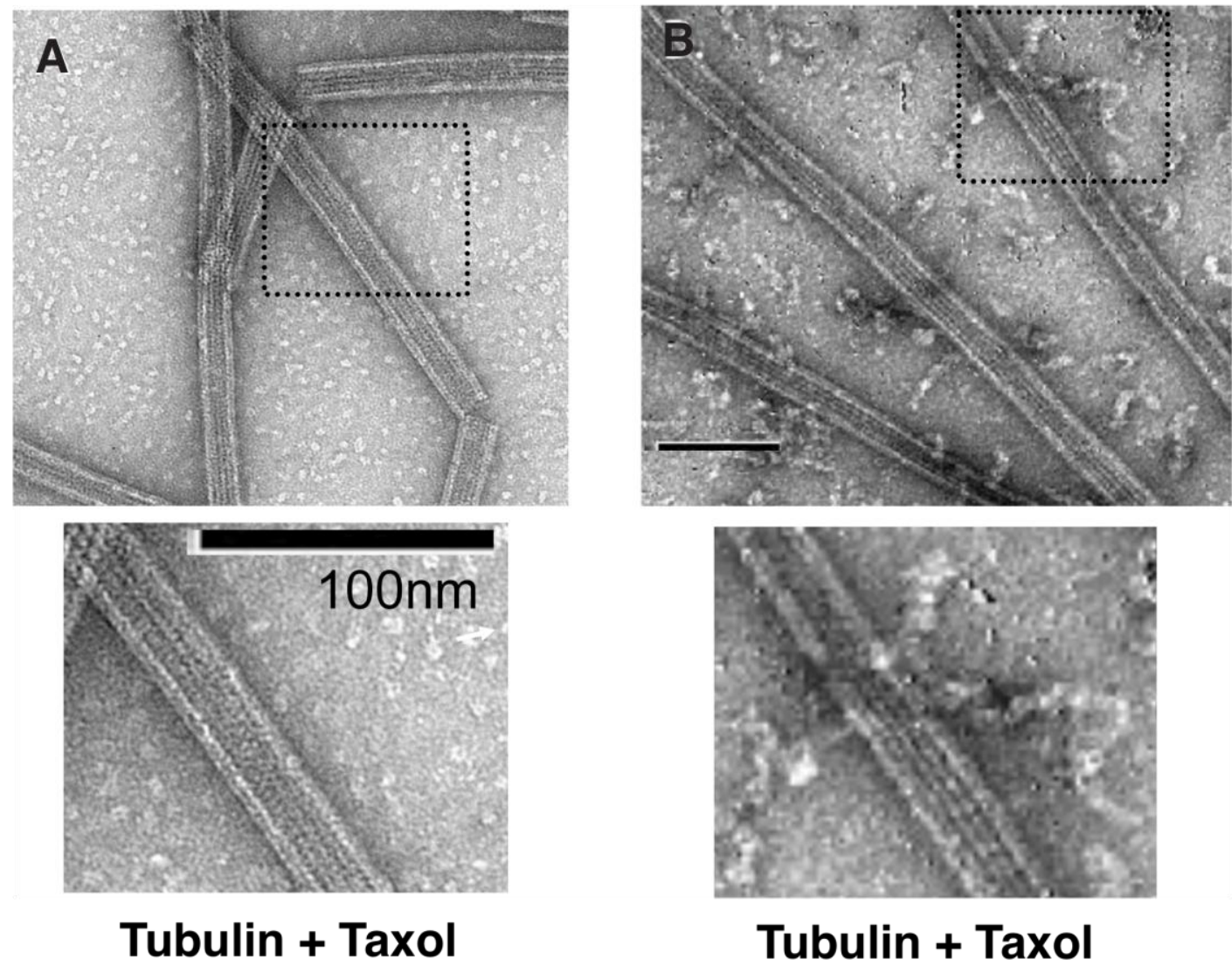

Tubulin + Taxol

+ xCep57R

Figure 2-3 


\section{Cep57R oligomerizes and binds to Cep57}

The Cep57 protein contains a multimerization domain that allows it to oligomerize both in vitro and in vivo (Bossard et al., 2003; Momotani et al., 2008). Given the apparent oligomerization we observed in our EM images of $\mathrm{xCep57R,} \mathrm{we} \mathrm{wanted} \mathrm{to}$ further examine the oligomerization properties of $\mathrm{xCep57R}$ in conditions that did not contain MTs. To do this, we expressed and purified recombinant 6-His tagged $\mathrm{xCep57R}$ protein. Eluted protein was run over a Superose 6 gel filtration column and the elution profile of the protein was measured. A protein doublet was seen in all xCep57Rcontaining fractions, presumably corresponding to a degradation product of the fulllength $\mathrm{xCep57R}$ protein. We found that the majority of $\mathrm{xCep57R}$ protein eluted from the column in a peak corresponding to the $158-\mathrm{kDa}$ column standard $\gamma$-globulin (Figure 24A). While such an elution profile could suggest a highly elongated shape for the $\mathrm{xCep57R}$ protein, it also suggests that the protein is capable of oligomerization.

Cep57R and Cep57 share regions of conserved sequence and common structural features, including predicted coiled-coil domains that can mediate protein-protein interactions (Derewenda et al., 2007). Therefore, we asked whether these two family members were capable of not only oligomerizing but also of binding to each other. To test this, we used the in vitro rabbit reticulocyte translation system to transcribe/translate radioactively labeled hCep57R and hCep57 proteins. Following translation of our two proteins (both alone and in combination), we incubated Protein-A beads bound with hCep57 family antibodies in our lysates (Antibody characterization is discussed later in this chapter - see Figure 2-7). Following incubation, beads were removed from the lysates and washed before being boiled in SDS-PAGE buffer and subjected to gel 
electrophoresis. Antibodies against hCep57 immunoprecipitated a majority of both hCep57 and hCep57R protein from the lysate (Figure 2-4B). Anti-hCep57R antibodies also pulled out appreciable amounts of both hCep57R and hCep57 from the lysate. Similar results were seen when hCep57R and hCep57 were co-translated in the same lysate. Therefore, while we cannot rule out the remote possibility of an intermediate binding protein existing in the reticulocyte lysate, these data suggest that Cep57R and Cep57 are capable of directly interacting with each other. In light of this finding, we asked if previous immunodepletions of $\mathrm{xCep57R}$ from Xenopus egg extracts had also removed the $\mathrm{xCep} 57$ protein. We attempted to generate polyclonal antibodies against recombinant $\mathrm{xCep57}$ protein to ask this question; however, the antibodies produced were of poor quality and could not be used to test this hypothesis. 
Figure 2-4: Cep57R oligomerizes and directly binds Cep57 in vitro

(A) Elution profile of $100 \mu \mathrm{g}$ recombinant full-length $\mathrm{xCep} 57 \mathrm{R}$ protein run over a

Superose 6 gel filtration column. One $\mathrm{mL}$ fractions were collected, TCA-precipitated and re-suspended in 2X SDS-PAGE sample buffer. Samples were then subjected to SDS-

PAGE followed by Coomassie staining to identify xCep57R protein. The peak of $\mathrm{xCep57R}$ protein elutes from the column in the same fractions as the $158-\mathrm{kDa} \gamma$-globulin control. None of the protein elutes from the column in the void volume. Load corresponds to $3 \mu \mathrm{g}$ of protein. (B) hCep57 and hCep57R were transcribed and translated in vitro using rabbit reticulocyte lysate. Radioactive methionine was added to the IVT lysate to label newly translated protein. Control IgG, hCep57, or hCep57R antibodies were then coupled to Protein A beads and used to immunoprecipitate the labeled proteins from the lysate. Translation of luciferase protein is a control to demonstrate efficacy of reticulocyte IVT system. 
A

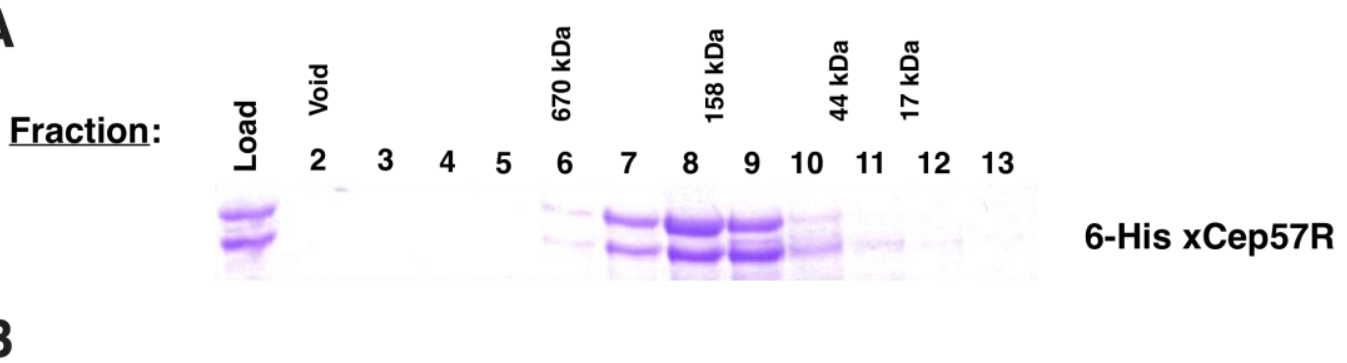

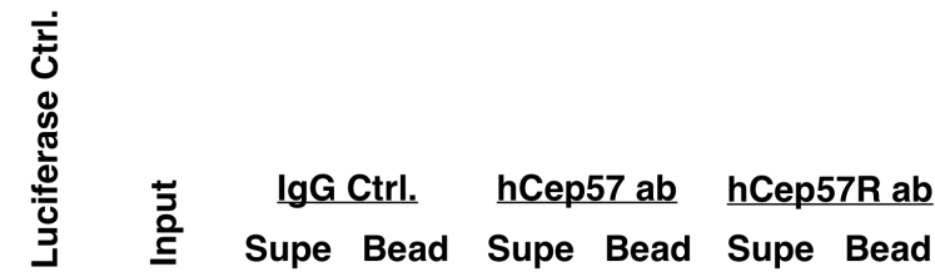
hCep57 IVT

hCep57R

IVT

hCep57R + hCep57

IVT

Figure 2-4 


\section{Cep57R and Cep57 interact with distinct sets of mitotic proteins}

During mitosis, mCep57 localizes to microtubules and spindle poles (Momotani et al., 2008). We therefore asked if mCep57 interacts with other mitotic proteins, including kinetochore components. We generated two GST-tagged recombinant mCep57 proteins, GST N-mCep57 and GST C-mCep57. GST N-mCep57 corresponds to amino acids 1-331 of the protein; GST C-mCep57 corresponds to amino acids 332-500. Proteins were bound to GST sepharose beads and incubated in clarified mitotic Xenopus egg extracts. Incubations were performed at $4^{\circ} \mathrm{C}$, where microtubules are depolymerized and no microtubule-based interactions can occur. Beads were washed and re-suspended in SDS-PAGE buffer. Proteins were boiled off the beads and supernatants were run out on an SDS-PAGE gel for Western blot analysis. We found that the C-terminus of the mCep57 protein pulled down numerous kinetochore/centromere components, including Zwint, CLIP-170, and Aurora B (Figure 2-5A). Both halves of the protein also weakly interacted with $\gamma$-tubulin. Interestingly, the C-terminus of mCep57 interacted with all five members of the HURP complex. This complex includes two MAPs (TPX2 and xMAP215), a centrosomal mitotic kinase (Aurora A), a microtubule plus-end directed motor protein (Eg5) and a microtubule-associated protein (HURP) that localizes close to kinetochores in response to high levels of Ran-GTP (Koffa et al., 2006). These interactions suggest that Cep57 could also function at both ends on the mitotic spindle, either by itself or in conjunction with Cep57R.

We next asked if Cep57R also interacted with HURP complex members. Fulllength GST-xCep57R protein was generated, in addition to numerous GST-tagged fragments of the protein. Proteins were bound to GST sepharose beads and incubated in 
clarified mitotic Xenopus egg extract at $4^{\circ} \mathrm{C}$ for 90 minutes. We were able to detect interactions between full-length xCep57R and Aurora A, HURP, CLIP-170 and $\gamma$-tubulin (Figure 2-5B). However, we were unable to detect interactions between xCep57R and either xMAP215 or Ndc80. While the majority of the interactions with Cep57 are mediated by its C-terminal half of the protein, only Aurora A and TPX2 show a strong interaction with the C-terminus of Cep57R. We were able to identify amino acids 425 488 of the protein as the primary interaction domain for Aurora A and TPX2. While the C-terminal half of Cep57 interacts with HURP and CLIP-170, it is the N-terminal half of Cep57R that facilitates interactions with these two proteins. Interestingly, full length Cep57R interacts with different splice variants of both HURP and CLIP-170 proteins compared with the N-terminal half of Cep57R alone. While splice variants of these two Xenopus proteins have been previously reported (Emanuele et al., 2005; Koffa et al., 2006), the significance of this alternate binding pattern remains unclear. 
Figure 2-5: Cep57 and Cep57R interact with numerous mitotic proteins

(A) N-terminally tagged GST-N mCep57 (corresponding to amino acids 1-331 of the protein) and GST-C mCep57 (corresponding to amino acids 332-500 of the protein) proteins bound to GST-sepharose beads were incubated in clarified mitotic Xenopus egg extracts at $4^{\circ} \mathrm{C}$ for 90 minutes. Beads were then removed from the extract, washed and re-suspended in 2X SDS-PAGE sample buffer. Samples were run out by SDS-PAGE and probed for the indicated proteins. GST Alone is a control for non-specific binding to GST-sepharose beads. For (A), Ko Momotani generated the recombinant proteins; Mike Emanuele performed the GST pull downs and Western blots. (B) N-terminally GSTtagged fragments of the $\mathrm{xCep57R}$ protein were bound to GST-sepharose beads and incubated in clarified mitotic Xenopus egg extracts for 90 minutes at $4^{\circ} \mathrm{C}$. Beads were then removed from the extract, washed and re-suspended in 2X SDS-PAGE sample buffer. Samples were run out by SDS-PAGE and probed for the indicated proteins. GST Alone is a control for non-specific binding to GST-sepharose beads. (C) Crude mitotic Xenopus egg extracts were supplemented with rhodamine-labeled tubulin, constitutively active Ran protein (RanQ69L) and either control anti-IgG or anti-xCep57R antibodies and incubated at room temperature for 60 minutes. Following incubation extracts were squash-fixed onto cover slips and processed for visualization of tubulin-based structures. The image contains two bipolar Ran-induced spindles and one Ran-induced aster. One hour following xCep57R antibody addition, extracts contained 45\% Ran-induced spindles and 55\% Ran-induced asters. 

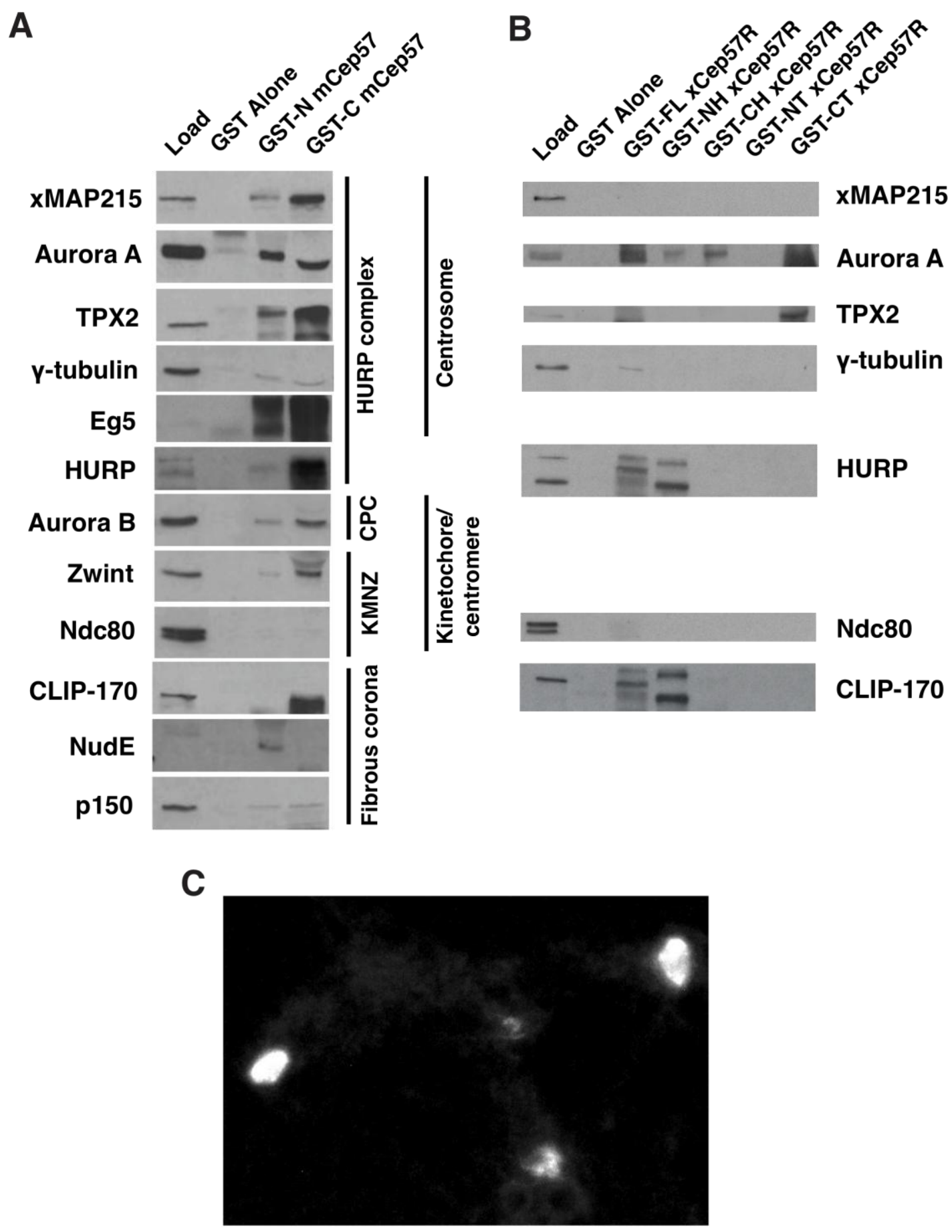

Figure 2-5 


\section{Cep57R and Cep57 are in vitro kinase substrates}

As xCep57R interacted strongly with Aurora A, we next asked whether Cep57R was an Aurora A substrate. Recombinant xCep57R proteins were incubated with one of three mitotic kinases (recombinant Aurora A, recombinant Aurora B, or MPS1 immunoprecipitated from Xenopus extract), ATP and radioactively labeled phosphate for 10 minutes at room temperature. The kinase reaction was stopped by addition of $2 \mathrm{X}$ SDS-PAGE buffer, proteins were boiled and subjected to SDS-PAGE gel electrophoresis. Radioactive gels were then exposed on a phospho-imager. We find that both Aurora A and MPS1, but not Aurora B, are able to phosphorylate xCep57R in vitro (Figure 2-6A). MPS1 is capable of phosphorylating both the N- and C-terminal halves of xCep57R, suggesting the presence of multiple MPS1 sites on the protein. Aurora A primarily phosphorylates the N-terminal half of the protein, preferentially on an unstructured region of the protein corresponding to amino acids 1-50. Aurora B failed to phosphorylate xCep57R despite strongly phosphorylating the control substrate Histone H3. Therefore, we conclude that two mitotic kinases known to localize to centrosomes can phosphorylate $\mathrm{xCep57R}$ in vitro but a predominantly centromeric kinase cannot. Interestingly, mCep57 is a substrate for Aurora B kinase in vitro (Figure 2-6B), suggesting that regulation of these proteins may differ. 
Figure 2-6: Cep57 and Cep57R are phosphorylated by mitotic kinases

(A) Cartoon schematic of recombinant GST-tagged xCep57R proteins used for in vitro phosphorylation studies. (B) Indicated recombinant xCep57R proteins were incubated with ATP, radioactively labeled phosphate and one of three active kinases: MPS1 immunoprecipitated from mitotic Xenopus egg extract, recombinant Aurora A or recombinant Aurora B. Proteins were incubated with kinase for 10 minutes at room temperature before the reaction was stopped by addition of $2 \mathrm{X}$ SDS-PAGE sample buffer. Samples were run out on an SDS-PAGE gel before being exposed on a phosphoimager. (C) Cep57 protein was incubated with ATP, radioactively labeled phosphate and active Aurora B kinase for indicated amounts of time. Reactions were stopped by addition of $2 \mathrm{X}$ SDS-PAGE buffer to sample. Samples were then run out on an SDSPAGE gel. mCep57 protein was identified by Coomassie staining; Aurora B phosphorylation of mCep57 was identified by exposing gel on a phospho-imager. For (C), Ko Momotani produced the recombinant mCep57 protein and Mike Emanuele performed the in vitro kinase reaction. 
A
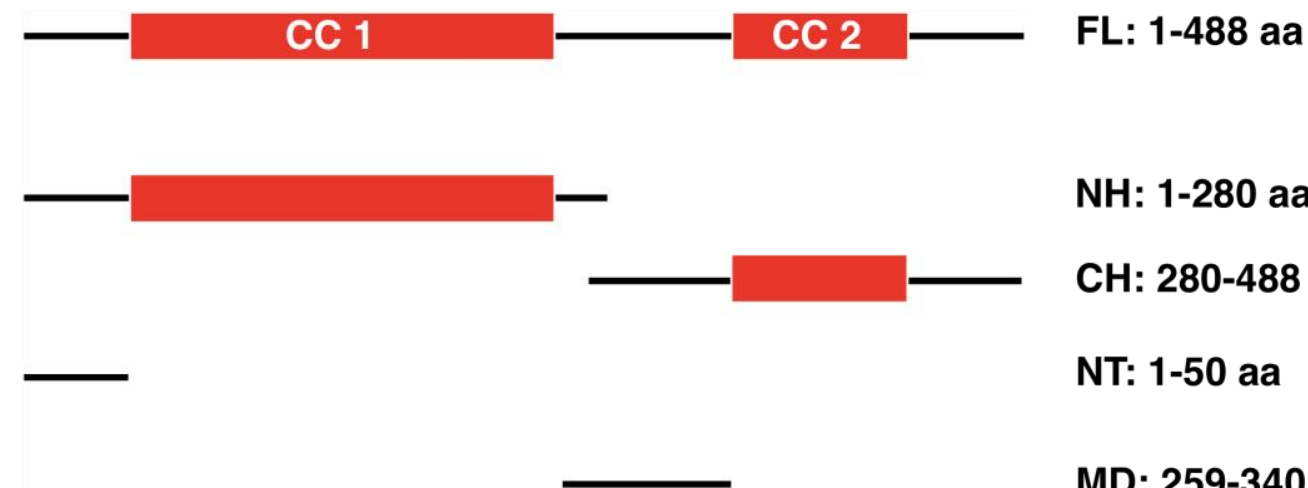

NH: 1-280 aa

CH: $280-488$ aa

NT: 1-50 aa

MD: 259-340 aa

— CT: $425-488$ aа

B

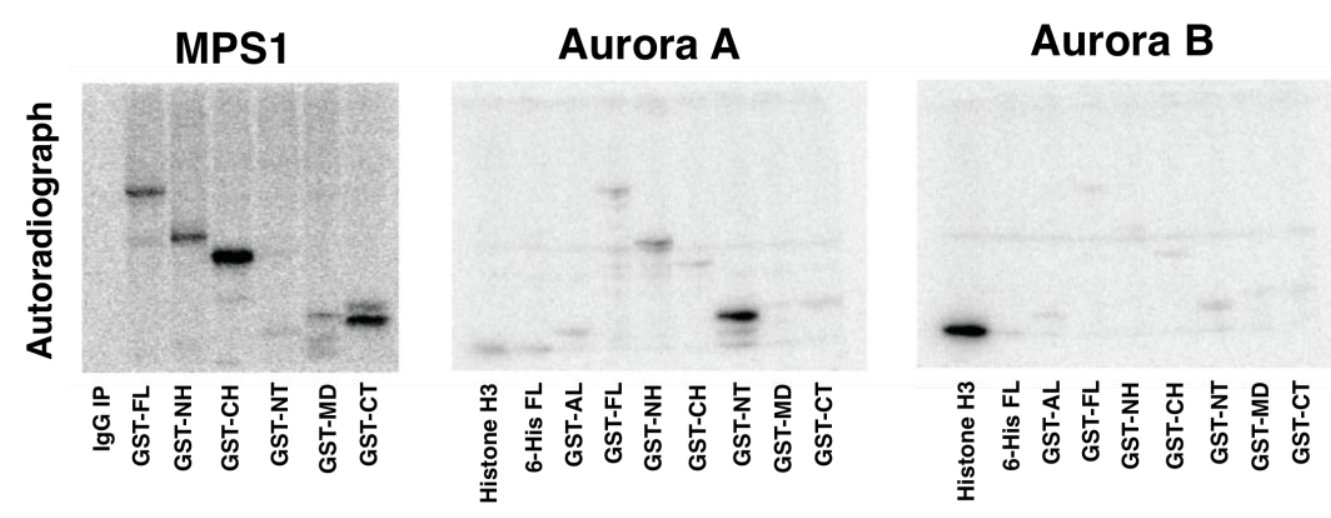

C

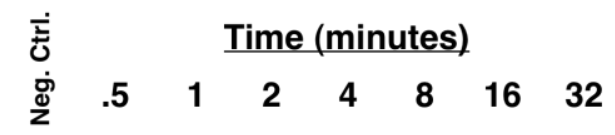

Aurora B activity

mCep57 protein

Figure 2-6 
xCep57R and HURP are functionally separable

The Xenopus HURP complex is formed in the presence of microtubules, Aurora A activity and high levels of RanGTP (Casanova et al., 2008; Koffa et al., 2006; Wong et al., 2008). Like TPX2, HURP has been identified as a target of both Ran and Aurora A (Gruss et al., 2001; Sillje et al., 2006; Wong et al., 2008). While HURP depletion from Xenopus egg extracts does not affect microtubule growth from purified centrosomes, depletion does abolish microtubule assembly that is induced by either constitutively active Ran (high RanGTP) or chromatin-containing beads (Casanova et al., 2008). During spindle bipolarization, addition of anti-HURP antibodies to egg extracts abolishes both Ran-mediated and chromatin/centrosome-mediated spindle formation (Koffa et al., 2006). In contrast, $x$ Cep57R inhibition abolishes anchorage of microtubules from sperm centrosomes but has no affect on Ran-induced microtubule asters (Emanuele and Stukenberg, 2007). Additionally, depletion of xCep57R from extracts abolishes chromatin/centrosome-mediated spindle formation. We therefore asked whether xCep57R inhibition affected Ran-induced spindle formation.

Constitutively active Ran (Ran Q69L) protein was added to mitotic Xenopus egg extracts to induce spindle formation (Carazo-Salas et al., 1999). Control IgG or antixCep57R antibodies were also added to the extracts upon introduction of Ran. After 60 minutes, extracts were fixed, spun onto cover slips and processed for indirect immunofluorescence. In a similar published experiment, addition of anti-HURP antibodies resulted in weakened Ran-aster formation and a failure of asters to form a bipolar spindle (90\% Ran asters, 10\% Ran-spindles) (Koffa et al., 2006). However, we found that spindle bipolarization was less impacted by addition of anti-xCep57R 
antibodies to Ran extracts. After 60 minutes, $45 \%$ of the tubulin structures identified were Ran-asters; 55\% were Ran-spindles (Figure 2-5C). We conclude that although xCep57R and HURP physically interact they are functionally separable. Notably, xCep57R does not require microtubules to interact with HURP complex members even though HURP complex formation requires microtubules. Additionally, in HeLa cells and in Xenopus egg extracts lacking microtubules, HURP has been shown to act independent of complex formation (Casanova et al., 2008; Wong et al., 2008). Therefore, if xCep57R functions in parallel with proteins such as HURP, TPX2 or Aurora-A it does not do so in the context of a HURP complex.

\section{Characterization of hCep57R and hCep57 antibodies}

We next asked whether Cep57 family function was conserved across vertebrate species. We began by raising polyclonal antibodies against the full-length hCep57R and hCep57 proteins. Affinity-purified antibodies against both proteins recognized bands at the predicted molecular weights $-55 \mathrm{kDa}$ for hCep57R and $57 \mathrm{kDa}$ for hCep57 (Figure 2-7A and 2-7B). However, both antibodies also recognize numerous cross-reacting bands. To ask if the 55-kDa and 57-kDa bands indeed corresponded to hCep57R and hCep57, we knocked down both endogenous proteins with siRNA oligo treatment. Western blots of HeLa cell lysates taken 48 hours post-transfection demonstrate efficient knockdown of both bands with the cross-reacting bands remaining unaffected (Figure 27A). Notably, the removal of one protein does not affect the stability of the other suggesting that these two proteins do not form a strong interaction in vivo. 
The relative non-specificity of our Cep57 antibodies made indirect immunofluorescence experiments more challenging. For example, staining patterns were sensitive to fixation method. When cells were fixed with paraformaldehyde followed by a short methanol treatment, hCep57R antibodies localized to spindle microtubules but also exhibited a high level of background cytoplasmic signal. In contrast, fixation of HeLa cells with ice-cold methanol resulted in prominent centrosome staining for both hCep57R and hCep57 (Figure 2-7D).

We asked if the centrosome-specific signal was a result of our antibody reacting with a Cep57 family member or with a cross-reacting band. To test this, we knocked down endogenous hCep57R and hCep57 with siRNA oligos. While we were unable to see a definitive loss of centrosome signal for hCep57R following siRNA-mediated knockdown (possibly due to another cross-reacting centrosome protein), we were able to demonstrate loss of hCep57 signal from centrosomes. In contrast, levels of p150, a member of the dynactin complex that also localizes to the centrosome, were unaffected (Figure 2-7E). 


\section{Figure 2-7: Characterization of hCep57 and hCep57R}

(A) Knockdown of hCep57 and hCep57R in HeLa cells. HeLa cells were transiently transfected with $75 \mathrm{nM}$ GAPDH (Mock), hCep57 or hCep57R siRNA oligos to knock down indicated proteins. An additional $100 \mathrm{nM}$ hCep57R condition was included in the experiment. Forty-eight hours following transfection, HeLa cell lysates were collected and overall protein concentrations were measured. $30 \mu \mathrm{g}$ of lysate for each condition was run out on an SDS-PAGE gel. Western blotting was performed to detect hCep57, hCep57R or tubulin protein levels in the indicated samples. (B) Uncropped gel images from the experiment described in (A). Note the presence of numerous cross-reacting bands. (C) HeLa cells were treated with $75 \mathrm{nM} \mathrm{GAPDH,} \mathrm{hCep57,} \mathrm{or} \mathrm{hCep57R} \mathrm{oligos} \mathrm{for}$ 48 hours to knock down the indicated proteins. Cells were then co-fixed with $2 \%$ paraformaldehyde and $0.5 \%$ Triton- $\mathrm{X}$ detergent and processed for indirect immunofluorescence. Samples were then scored for the percentage of mitotic cells containing multi-polar spindles. (D) Asynchronous HeLa cells were fixed using either ice cold $\mathrm{MeOH}(\mathrm{MeOH})$ or $2 \%$ paraformaldehyde followed by a post-fix with ice cold $\mathrm{MeOH}(\mathrm{Para}+\mathrm{MeOH})$ and processed for indirect immunofluorescence. Cells were immunostained for tubulin, hCep57R and the centromeric marker ACA. (E) Asynchronous HeLa cells were transiently transfected with siRNA oligos targeting GAPDH (Mock) or hCep57 to knock down the indicated proteins. Cells were fixed 48 hours later and immunostained for hCep57 and p150. 
A

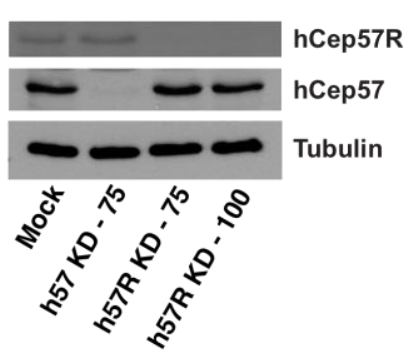

C

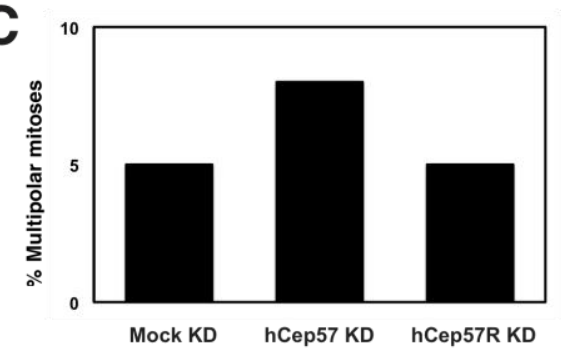

D
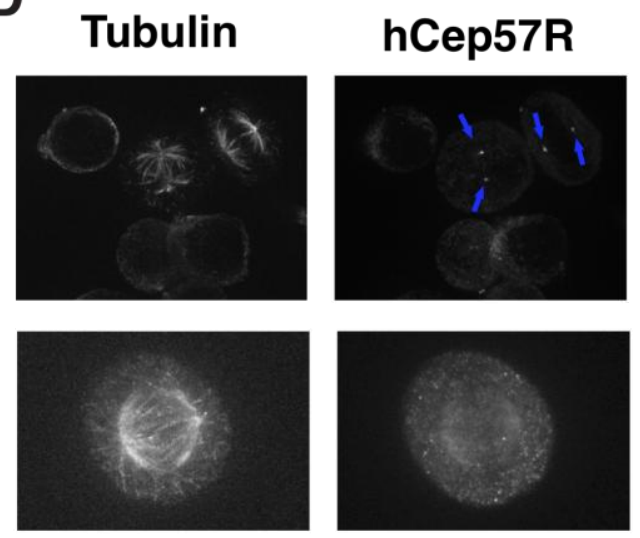

E

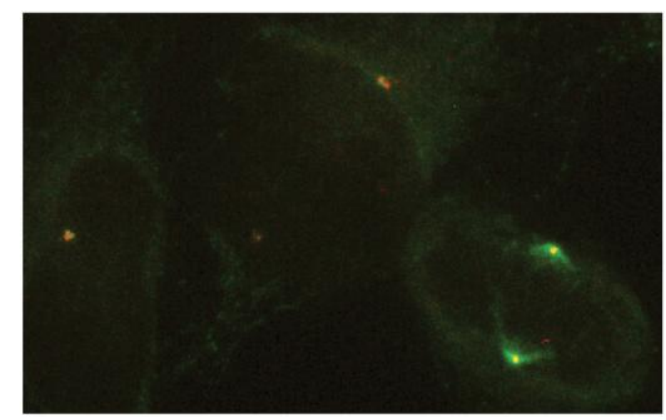

p150 hCep57

B
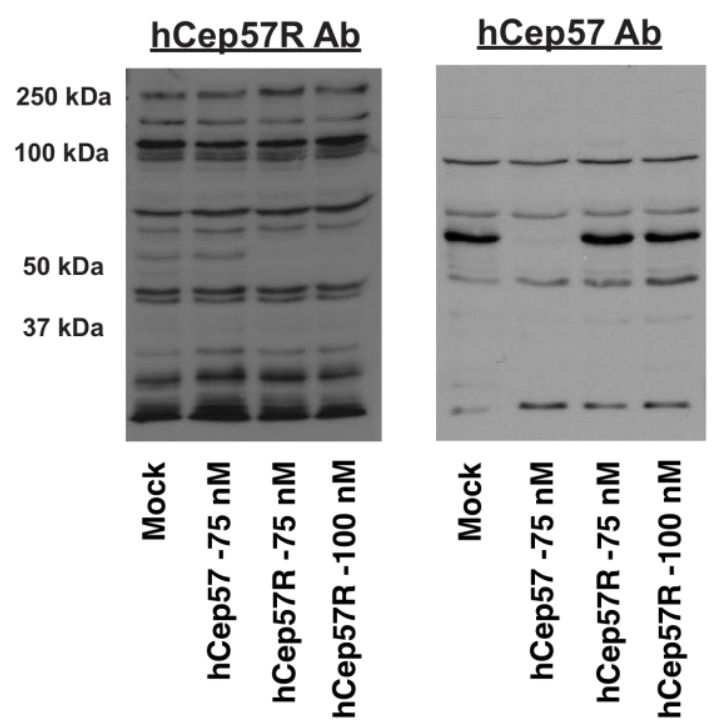

ACA
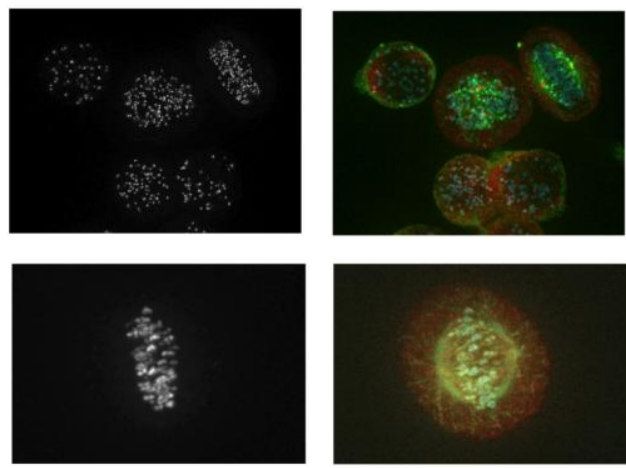

Para + $\mathrm{MeOH}$

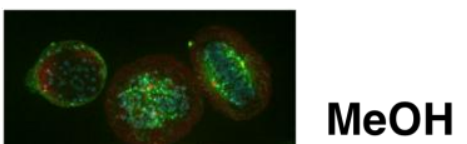

hCep57 siRNA - 48 hours

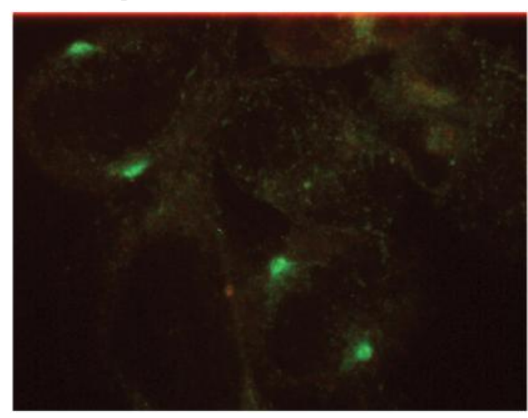

p150

Figure 2-7 


\section{Localization of hCep57R and hCep57 in HeLa cells}

As neither one of our hCep57 family antibodies was specific, we next employed an alternative strategy to examine the localization patterns of both proteins. GFP-tagged hCep57 and hCep57R were transiently transfected into HeLa cells, fixed 24 hours later and processed for indirect immunofluorescence. During interphase, we were able to detect both hCep57 and hCep57R at MTOCs, further confirming that both proteins are MTOC/centrosome components (Figure 2-8). We were unable to visualize any cells in mitosis, and also noticed an increased amount of cell death in cells transfected with GFPCep57 family constructs. Given Cep57's role in binding and bundling MTs when overexpressed (Momotani et al., 2008), we conclude that our inability to find a viable mitotic cell likely results from this bundling phenotype.

The primary motivation of our immunofluorescence and GFP-tagging experiments was to ask whether hCep57R, like xCep57R, was capable of localizing to kinetochores. Having failed thus far to answer to this question, we turned to examining Cep57R antibody staining on chromosome spreads. This method has previously been employed to visualize kinetochore proteins that are refractory to traditional immunofluorescence treatments (Porter et al., 2007). Chromosome spreads are generated by arresting tissue culture cells in mitosis, treating them with hypotonic media to induce swelling, and subjecting cells to a high-speed spin that lyses the cell and adheres the chromosomes to a cover slip. Indirect immunofluorescence can them be performed on these chromosomes. We were unable to detect kinetochore staining of hCep57R in our chromosome spreads (data not shown). Therefore, while both human and Xenopus 
Cep57R can bind microtubules and localize to centrosomes it remains an open question whether Cep57R is a conserved kinetochore protein.

\section{Phenotypes for hCep57R and hCep57}

We were able to take advantage of the robust knockdown of our hCep57R and hCep57 proteins to study the phenotypes generated upon removal of these two proteins from HeLa cells. Synchronized cells were transiently transfected with 75nM of GADPH (mock control), hCep57 or hCep57R siRNA oligos and subsequently released from their thymidine block. Twelve hours later cells were again placed in thymidine-supplemented media to generate a G1/S arrest, and 12 hours later cells were released into thymidinefree media. Cells were fixed 9 hours post-release (as the majority of the population was traversing mitosis) and processed for immunofluorescence. While we noticed a modest increase in multipolar spindles in cells with reduced levels of hCep57 protein, we did not observe any appreciable phenotypes in our hCep57R knockdown cells (Figure 2-7C). Similarly, co-knockdown of both Cep57R and Cep57 also failed to generate robust phenotypes (data not shown). While the modest phenotypes generated by hep57 knockdown are consistent with published reports of Cep57 function (Bossard et al., 2003; Meunier et al., 2009; Momotani et al., 2008; Ruiz-Miro et al., 2011), the lack of a robust Cep57R phenotype in human cells stands in contrast to what has been observed in Xenopus extracts following xCep57R inhibition (Emanuele and Stukenberg, 2007). 
Figure 2-8: Testing kinetochore localization of hCep57R and hCep57

(A) N-terminally GFP-tagged hCep57 and hCep57R proteins localize to MTOCs of interphase cells. Asynchronous cycling HeLa cells were fixed in ice-cold MeOH was processed for indirect immunofluorescence. Cells were immunostained for tubulin; hCep57 or hCep57R were identified by expressed GFP tag. Note that GFP signal has been false-colored to appear red. 
A
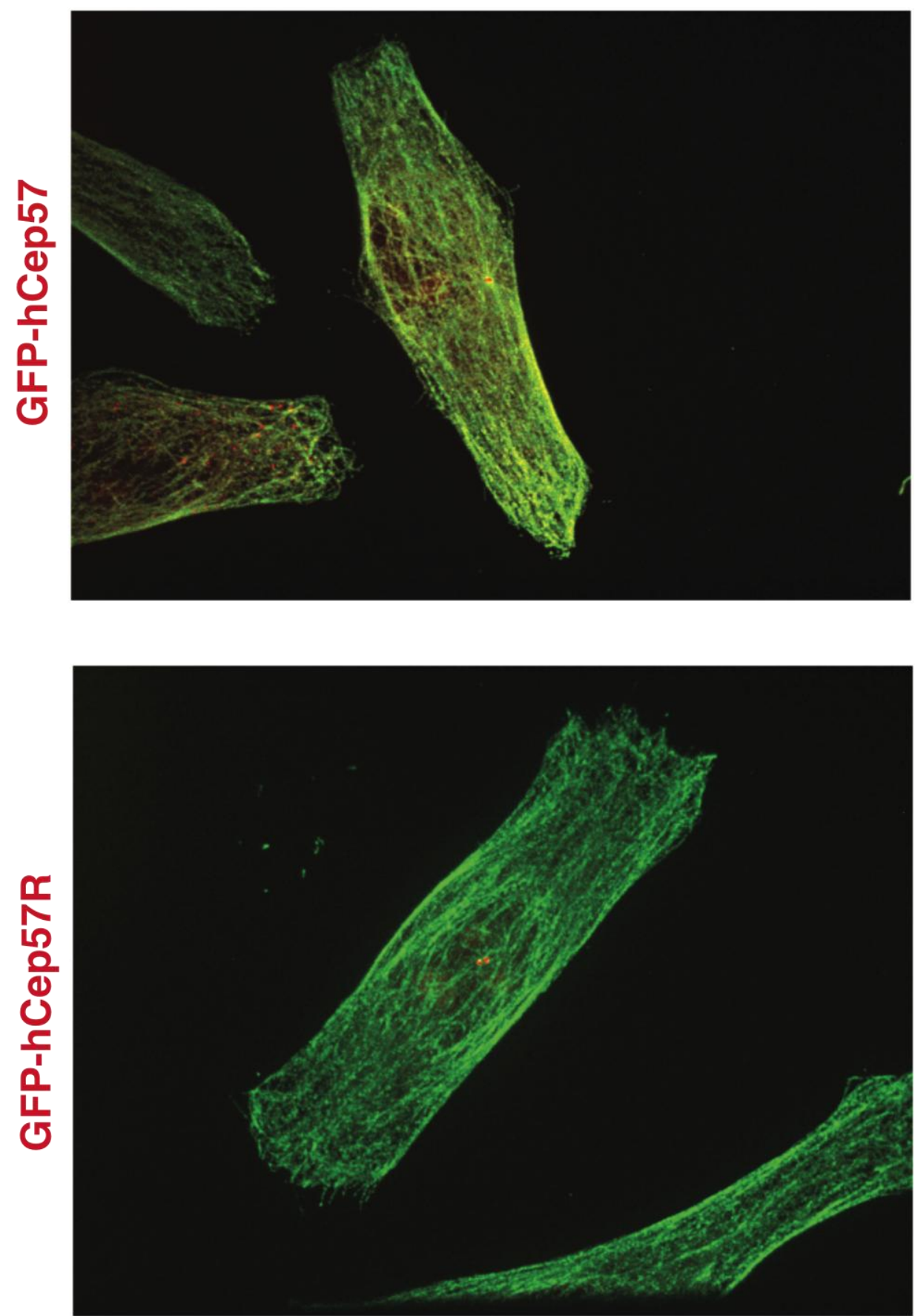

Figure 2-8 
$x$ Cep57R is a developmentally regulated protein

We next asked whether Cep57R might play a more prominent role in Xenopus egg extracts than in somatic human cells. There is precedent for this, as other mitotic proteins such as Ran, HURP and TD-60 have a more pronounced phenotype in meiotic systems (Breuer et al., 2010; Kalab et al., 2006; Mollinari et al., 2003; Rosasco-Nitcher et al., 2008). To address this question, we first examined $\mathrm{xCep57R}$ protein expression as a developing Xenopus embryo passed through a series of developmental stages. Briefly, equal amounts of protein harvested from Xenopus eggs or embryos at a series of developmental stages were run out on an SDS-PAGE gel and subjected to Western blot analysis. While kinetochore/centromere proteins such as Ndc80 and Dasra A are expressed at similar levels as development proceeds, expression of $\mathrm{xCep57R}$ is only elevated from the egg stage through Stage 9.5, which occurs slightly after the midblastula transition (Figure 2-9A). Between Stage 9.5 and Stage 10.5, levels of xCep57R are greatly reduced and do not recover. Therefore, it is possible that the requirements for Cep57R are greatest during the early stages of development.

In addition to being present in the cytoplasm of the egg, we also discovered a second source of $\mathrm{xCep57R}$ protein in our extract system. When we examined demembrenated sperm before they were incubated in an egg extract, we found by indirect immunofluorescence that $\mathrm{xCep57R}$ was already present on the sperm (Figure 2-9B). Other centrosome proteins such as pericentrin and $\alpha$-tubulin were also present while $\gamma$ tubulin was not, consistent with previous results (Stearns and Kirschner, 1994). Therefore, it appears that there are two sources of $\mathrm{xCep57R}$ protein in our extract-based experiments. This finding may explain the stronger phenotypes often observed when anti- 
$\mathrm{xCep57R}$ antibodies were added to extracts as opposed to when $\mathrm{xCep57R}$ was

immunodepleted (Emanuele and Stukenberg, 2007). However, the relative contribution made by these two sources of $\mathrm{xCep57R}$ remains undetermined. 
Figure 2-9: Characteristics of $x$ Cep57R during development

(A) Extracts from either an unfertilized egg or embryos in the indicated developmental stages were run out on an SDS-PAGE gel and Western blots were performed to probe for the indicated proteins. A cross-reacting band serves as an internal loading control. (B) Demembrenated sperm were diluted ten-fold in permeabilization buffer containing 1 $\mu \mathrm{g} / \mathrm{mL}$ DAPI as previously described (Stearns and Kirschner, 1994) before being spun onto cover slips, fixed for 5 minutes in ice-cold $\mathrm{MeOH}$, and stained for the indicated antigens. 
A

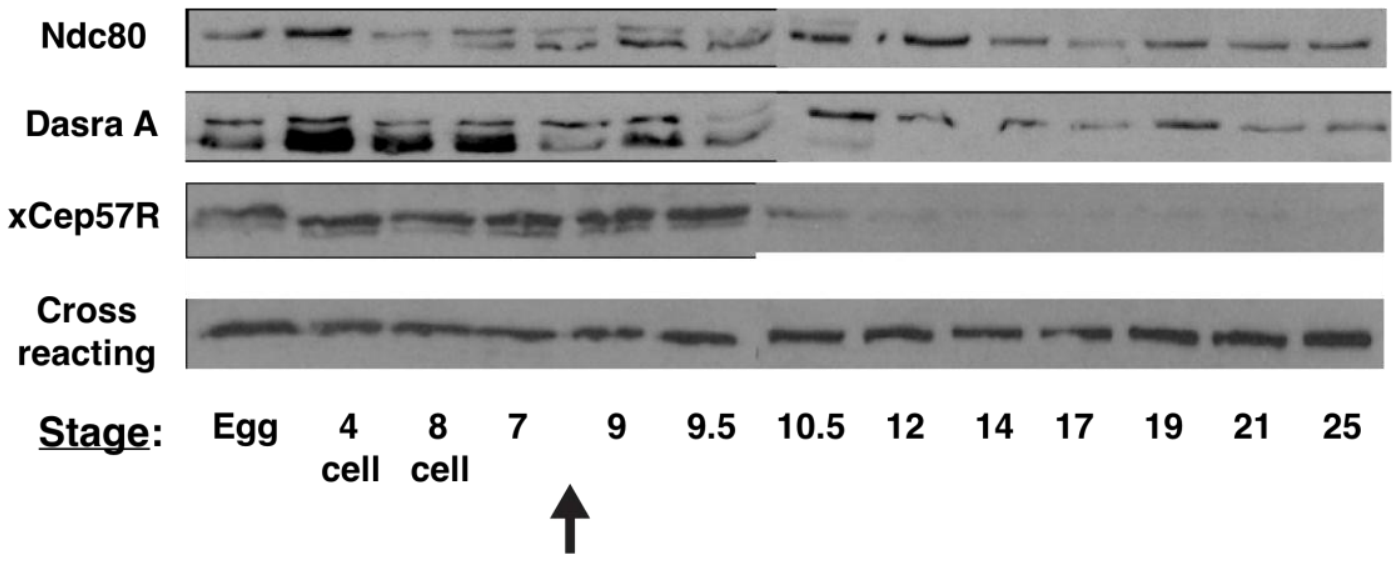

Mid-blastula transition

B
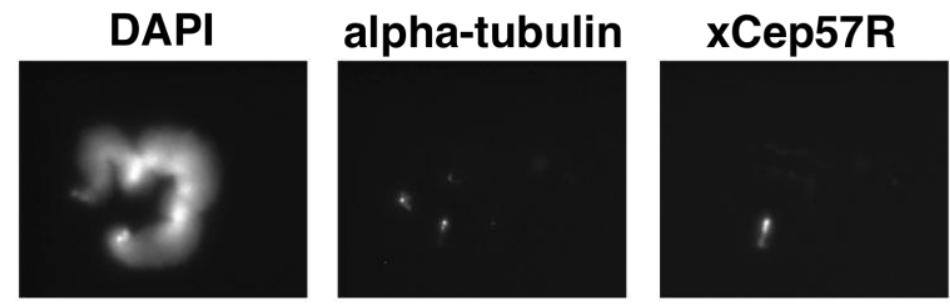

Merge
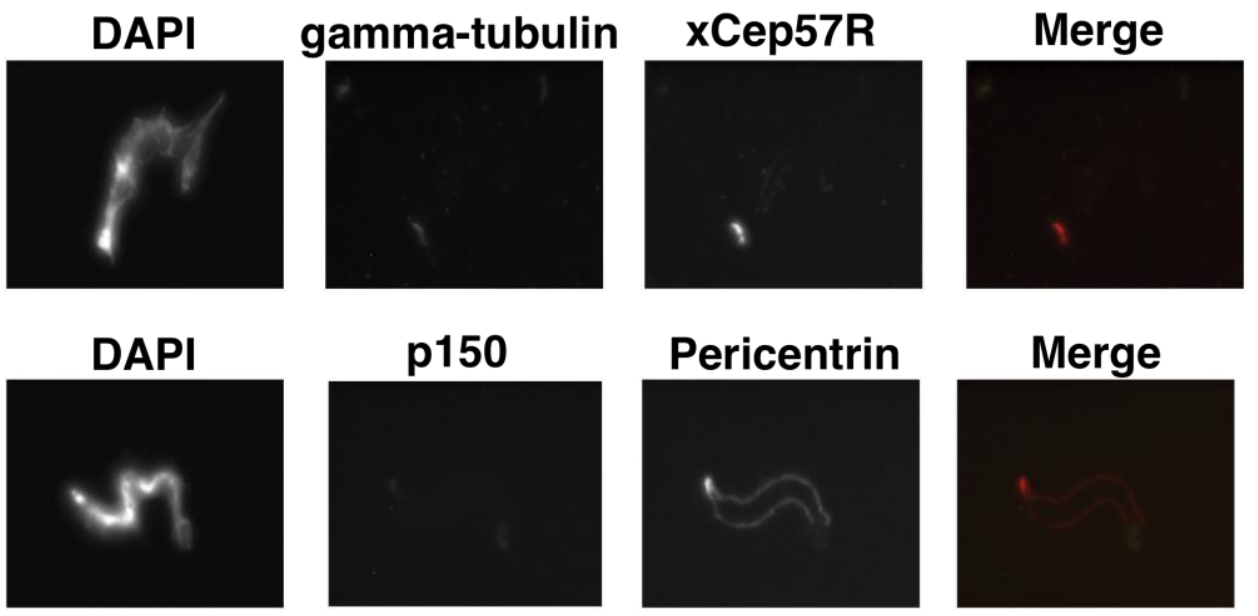

Figure 2-9 


\section{Discussion}

We have built on our earlier studies of Cep57R by demonstrating that it is capable of directly binding microtubules in vitro but incapable of forming rings around microtubules. In the absence of microtubules, Cep57R can both oligomerize and bind the closely related family member Cep57. Cep57R interacts with a number of additional mitotic proteins, including most members of the HURP complex. Like its binding partner HURP, Cep57R interacts with and is phosphorylated by the Aurora A kinase. However, unlike HURP we find that Cep57R is not regulated by Ran. Cep57R protein levels are sharply reduced in frog embryos at a developmental stage when other key mitotic factors continue to be expressed. While depletion of $\mathrm{xCep57R}$ in Xenopus egg extracts results in a robust phenotype, knockdown of hCep57R in HeLa cells does not. Therefore, while $\mathrm{xCep57R}$ is important during early development, many questions remain regarding overall Cep57R function.

The protein encoding $\mathrm{xCep57R}$ was initially identified as a candidate Dam1 vertebrate due to weak sequence similarity with 90 amino acids of the Dam1 protein. Originally called xCep57, additional sequence analysis discovered a second family member that was more closely related to hCep57 and mCep57. Therefore, the candidate Dam1 homolog was later re-classified as xCep57R (for xCep57 Related). Strong sequence conservation exists between Cep57R and Cep57 in the two predicted coiled-coil regions but there is divergence in the three small unstructured regions that comprise the remainder of both proteins. While Cep57R and Cep57 are capable of directly binding to each other, siRNA mediated knockdown of one family member does not affect the 
stability of the other. This suggests that Cep57R and Cep57 do not exist in a tightly associated complex in vivo.

Although Cep57R and Cep57 are both capable of directly binding microtubules and localizing to both centrosomes and spindle microtubules, the two proteins appear to have different functions. While Cep57 strongly interacts with all five members of the HURP complex, Cep57R does not. Cep57 is an in vitro Aurora B target, but Cep57R is phosphorylated by Aurora A and MPS1. A slight multipolar phenotype is observed following knockdown of Cep57 in HeLa cells, but no multipolar phenotype is seen for Cep57R. Therefore, these family members seem unlikely to be functionally redundant. Numerous interactions with members of the HURP complex originally led us to ask whether either Cep57 or Cep57R functioned as part of this complex. Several lines of evidence now argue against this. First, Cep57 can interact with HURP complex members independent of MTs, despite evidence demonstrating that MTs are required to facilitate HURP complex formation (Casanova et al., 2008). Cep57R is also capable of interacting with some but not all members of the complex. Additionally, while HURP complex function is subject to Ran regulation we have been unable to demonstrate a similar regulation of Cep57R function. (The effect of Ran on Cep57 function remains untested). In both HeLa cells and in Xenopus egg extracts, HURP has been shown to function outside the context of the HURP complex. Therefore, interactions of Cep57R or Cep57 with individual HURP complex members may still be important even if an entire HURP complex is not formed.

Knockdown of hCep57 in HeLa cells generates subtle phenotypes, consistent with what other groups have reported for Cep57 function. However, the discrepancy between 
Cep57R phenotypes in Xenopus egg extracts and HeLa cells is striking. There are many potential explanations for the discrepancy. As previously mentioned Cep57R may simply be a protein relied on during early development. Additionally the length of spindles in Xenopus egg extracts is far greater than in a HeLa cell, and mitosis occurs rapidly in early Xenopus embryo development (Dumont et al., 2007; Wuhr et al., 2008). Therefore, it is possible that $\mathrm{xCep57R}$ is required in this context to address additional geometric or temporal considerations.

A second possibility is that immunodepletions of xCep57R are pulling down not only xCep57R from extracts but also xCep57 (or other unknown proteins), and this combined loss was responsible for generating the observed phenotypes. We attempted to test this hypothesis by generating polyclonal antibodies to xCep57, but our antibodies were not of sufficient quality to address this question. In the future, another way to test this idea would be to pull down recombinant tagged xCep57 protein from a Xenopus egg extract and use our $\mathrm{xCep57R}$ antibody to blot for $\mathrm{xCep57R}$ in the material pulled out by the beads. Currently in our lab we have the $\mathrm{N}$-terminal half of the xCep57 protein with a 6-His tag at its $\mathrm{N}$-terminus. While this protein may prove useful, it seems a better idea would be to first generate a full-length xCep57 protein for this experiment. Therefore, if a potential xCep57R interacting domain were to interact with the C-terminal half of xCep57 this interaction would be captured.

A third possibility is that the antibody used to study $\mathrm{xCep} 57 \mathrm{R}$ function is recognizing a cross-reacting protein (or proteins) that is responsible for generating the observed phenotypes. Characterization of the xCep57R antibody showed a prominent cross-reacting band in egg lysates, though this band was not lost following 
immunodepletion of the extract (Emanuele and Stukenberg, 2007). The identity of this band remains unknown. It is important to note that all phenotypes ascribed to $\mathrm{xCep57R}$ were generated following the use of this antibody. Therefore, before proceeding with additional investigation of Cep57R function it seems important to first re-capitulate the observed phenotypes without use of that antibody. To this end, our lab has begun using morpholino oligos to knock down xCep57R in live Xenopus embryos. Only modest phenotypes have been generated to date, but it should be noted that we have not been able to reliably ascertain knockdown levels (P. Amman, unpublished data). Until we can verify efficient knockdown we cannot make any strong conclusions about these experiments.

It remains uncertain whether Cep57R is a conserved kinetochore protein. We and others have been unable to identify a kinetochore-specific population of Cep57R in HeLa cells (Welburn et al., 2009). In chicken DT-40 cells ggCep57R has been identified as a kinetochore-associated protein, but no functional analysis of ggCep57R has been performed to date (Ohta et al., 2010). It may be the case that Cep57R only acts as a kinetochore protein in Xenopus, but given the concerns over antibody specificity another approach may be to attempt to localize GFP-tagged Cep57R to kinetochores in a live frog embryo. However, before such an experiment is performed it would be beneficial to consider the level of GFP-Cep57R being expressed, given the microtubule bundling phenotype observed upon Cep57 overexpression.

At present there are little data implicating Cep57R misregulation in the development of disease. Since Cep57R (and Cep57) function have not been extensively studied, it is possible that additional implications for one or both of these proteins in 
disease may be forthcoming (Snape et al., 2011). However, given the fact that somatic cells are largely unperturbed by Cep57R/Cep57 knockdown, it also remains possible that these proteins are not critical during a vertebrate mitosis or that a functionally redundant protein exists. Finally, both proteins are expressed not only in mitosis but also in interphase. Given the trafficking defects that have been identified in Cep57 knockdown cells, future studies must determine whether any identified disease state was caused by protein disruption during interphase or mitotis. 


\section{Acknowledgements}

We thank Eliza Zylkiewicz and Dan Matson for critical reading of this chapter. Ko Momotani from the Somlyo laboratory generated the recombinant mCep57 proteins used in this chapter; Mike Emanuele used these proteins to perform GST pull downs and in vitro kinase reactions. Weijie Lan generated sequence alignments of Cep57 family members. Albina Orlova from the Egelman laboratory performed the negative EM staining of xCep57R. 


\section{Chapter 3: THE NDC80 COMPLEX: INTEGRATING THE KINETOCHORE'S MANY MOVEMENTS}

This chapter is based on the following publication:

Tooley, J and Stukenberg, PT. "The Ndc80 complex: integrating the kinetochore's many movements" Chromosome Research. (2011) 19, 377-391. 


\section{Abstract}

The Ndc80 complex lies at the heart of the kinetochore, a large protein machine that accurately segregates chromosomes during cell division. The Ndc80 complex has structural roles in assembling the kinetochore, but also functions to congress chromosomes and to signal the spindle checkpoint. It directly binds to microtubules and is currently the best candidate for the long-sought protein that couples microtubule depolymerization to chromosome movement. A combination of structural and genetic data have recently converged to generate the first models for this fascinating motor activity. Additionally, recent data point to an increasingly dynamic role for Ndc80 in the kinetochore - one which involves not only simple binding to microtubules but also shifts in complex shape and its location within the overall kinetochore structure. In this review we discuss recent advances in our understanding of the Ndc80 complex and address future areas of research. 


\section{Mitosis and Kinetochores}

A mitotic spindle segregates replicated genomes into two daughter cells.

Microtubules form the mitotic spindle and make bipolar attachments to each chromosome through complex protein machines known as kinetochores. Mammalian kinetochores are comprised of more than 100 proteins which coordinate numerous activities including nucleation and capture of microtubules, regulation of microtubule dynamics, powering and coordinating chromosome movements and integrating cell cycle progression with kinetochore microtubule attachments (Cheeseman and Desai, 2008; Musacchio and Salmon, 2007; Stukenberg and Foltz, 2010; Tanaka and Desai, 2008). At the center of all of these activities is a kinetochore's ability to correctly link spindle microtubules to mitotic chromosomes.

Classic in vitro experiments by Koshland, Mitchison and Kirschner demonstrated that kinetochores could move isolated chromosomes on microtubules in the absence of ATP (Koshland et al., 1988). These data suggested that chromosomes are not powered by traditional ATP-driven motors, but rather by the energy stored in the dynamically unstable microtubule. This model was rigorously debated since some of the first kinetochore proteins identified were kinesins and dynein (Wordeman et al., 1991; Yen et al., 1992). Current models now suggest that ATP-driven motors play important roles in generating bipolar kinetochore attachments and coordinating spindle checkpoint signaling. However, ATP-driven motors are not required to align most chromosomes or to segregate chromatids in anaphase - reinvigorating the concept of depolymerizationcoupled movement (Howell et al., 2001; Putkey et al., 2002). Characterization of the Ndc80 complex in numerous model organisms has shown that this four-protein complex 
is essential for chromosome segregation in all eukaryotes. More importantly, it has a structure and activities consistent with a coupler that can harness the energy from a depolymerizing microtubule to perform the work of moving chromosomes.

\section{The early years - Discovery and Phenotypes}

Lee and colleagues first made the connection that proteins of the Ndc80 complex were critical for kinetochore movements. They isolated the Hec1/Ndc80 subunit in a twohybrid screen with the Retinoblastoma tumor suppressor and named the human protein Hec1 (for $\underline{H i g h l y}$ Expressed in $\underline{\text { Cancer }} \underline{1}$ ) (Chen et al., 1997). Using a monoclonal antibody against Hec1 they demonstrated that the protein localized to kinetochores. Injection of this antibody blocked mitotic chromosome movements in human cells. The budding yeast homolog was later identified as a contaminant in a spindle pole body preparation (Wigge et al., 1998). Since the phenotypes of temperature sensitive mutants were similar to those of $\mathrm{Ndc10}$, an established kinetochore protein, the budding yeast protein was named Ndc80. The budding yeast complex containing Ndc80 and three other tightly associated proteins was later purified. Two co-purifying proteins were believed to be members of the Spindle Pole Complex and were named Spc25 and Spc24 (Wigge and Kilmartin, 2001). The fourth protein, Nuf2, had previously been identified in a separate spindle pole body preparation (Osborne et al., 1994). While a role for these proteins at the spindle pole/centrosome is dubious, it is clear that all of the components of the $\mathrm{Ndc} 80$ complex are essential for kinetochore function in eukaryotes. All four proteins are able to ChIP to centromeres and are critical for chromosome segregation. The yeast Ndc80 complex also is required to generate the spindle checkpoint signal (Janke et al., 2001). 
Discovery of the vertebrate complex soon followed in a flurry of papers. These papers established that the four-protein Ndc80 kinetochore complex is conserved from yeast to humans and that the complex is highly elongated, consistent with its predicted coiled-coil structure (Bharadwaj et al., 2004; DeLuca et al., 2005; DeLuca et al., 2003; DeLuca et al., 2002; Desai et al., 2003; Hori et al., 2003; Martin-Lluesma et al., 2002; McCleland et al., 2003; McCleland et al., 2004). It is required for chromosome movements and kinetochore microtubule attachments in all species. The vertebrate complex also is required to generate spindle checkpoint signals, although the requirement is highly sensitive to the degree of knockdown and shows some species variability. Early experiments further demonstrated that the complex plays a structural role in the kinetochore, as a number of outer kinetochore proteins could not properly assemble on kinetochores following Ndc80 knockdown (Ciferri et al., 2007; Hanisch et al., 2006; McCleland et al., 2003; McCleland et al., 2004; Ohta et al., 2010). This made it impossible to assign a direct role for the complex in the strong phenotypes generated by its knockdown. Later rescue experiments using separation of function mutants of the Hec1/Ndc80 subunit established that the complex does indeed play a direct role in facilitating chromosome movements (Guimaraes et al., 2008; Miller et al., 2008).

\section{Structure and function studies}

Hydrodynamic analysis and biochemical reconstitution of the $S$. cerevisiae, $C$. elegans, Xenopus laevis and human Ndc80 complexes opened the door to advances in our understanding of complex structure. The Ndc80 complex exists in a 1:1:1:1 stoichiometry with a calculated molecular weight of $\sim 170-190 \mathrm{kDa}$ (Cheeseman et al., 2006; Ciferri et al., 2005; Ciferri et al., 2008; McCleland et al., 2004; Wei et al., 2007; Wei et al., 2005). 
All four complex members co-purify with high affinity in vitro, suggesting a tight interaction (Cheeseman et al., 2004; McCleland et al., 2004; Wigge and Kilmartin, 2001). Rotary shadow EM and AFM demonstrated that the Ndc80 complex contains two globular domains at either end of a $\sim 57 \mathrm{~nm}$ highly elongated coiled-coil (Ciferri et al., 2005; Ciferri et al., 2008; Wei et al., 2006; Wei et al., 2005). The extended shape of the complex leaves it capable of spanning the distance between the electron-dense inner and outer regions of the kinetochore (Cleveland et al., 2003). At one end of the complex, the globular domains of Spc24 and Spc25 dimerize to form a "receptor" connecting the Ndc80 complex to more centromere-proximal elements of the kinetochore (Petrovic et al., 2010; Wei et al., 2006). At the other end of the complex, the Hec1/Ndc80 and Nuf2 globular domains dimerize to form a dual calponin homology domain (CHD) (Ciferri et al., 2008; Wei et al., 2007), a protein fold found in other microtubule and actin-binding proteins (Sjoblom et al., 2008). The Hec1/Ndc80 protein also contains an unstructured tail at its $\mathrm{N}$-terminus that ranges from 80-112 amino acids, depending on the species. The two globular domains are connected by the coiled-coil regions contributed by each protein. These regions allow the two dimers to tetramerize and to form the dumb-bell shape that characterizes this complex (Ciferri et al., 2008). The coiled region of the Hec1/Ndc80 protein is interrupted by an unstructured "hinge" region which allows the central shaft to bend in vitro (Wang et al., 2008) and is postulated to also permit complex flexibility in vivo (Wan et al., 2009) (Figure 3-1).

The MT binding activity of the Ndc80 complex resides in the Ndc80/Nuf2 globular region. There is a bipartite MT binding motif, as both the N-terminal tail and the $\mathrm{CHD}$ of Hec1/Ndc80 can bind microtubules in vitro and each contributes to the overall 
binding affinity (Cheeseman et al., 2006; Ciferri et al., 2008; Miller et al., 2008; Wei et al., 2007). It is currently unclear if the Nuf2 CHD contacts microtubules or simply regulates the Hec1/Ndc80 CHD (Alushin et al., 2010; Wilson-Kubalek et al., 2008). One possible role of the Nuf 2 CHD could be to form a dual CHD within the Ndc80 complex. A single Hec1 CHD cannot bind microtubules in vitro while the unstructured tail can (Miller et al., 2008). It is only when the Hec1/Ndc80 CHD is paired with the Nuf2 CHD that microtubule binding occurs. A similar effect is seen with the plus-end MT binding protein EB1, which cannot bind microtubules effectively unless dimerized (Zimniak et al., 2009). Dual CHD requirements also exist for actin-binding proteins such as $\alpha$-actinin (Sjoblom et al. 2008). 
Figure 3-1: Structure of the Ndc80 complex

The four-member Ndc80 complex consists of Hec1/Ndc80, Nuf2, Spc25 and Spc25. The highly elongated complex has a calculated molecular weight of 170-190 kDa and a length of $57 \mathrm{~nm}$. The globular N-terminal domains of Hec1/Ndc80 (blue) and Nuf2 (yellow) form a dual calponin homology domain (CHD), and the Hec1/Ndc80 subunit also contains an unstructured tail at its $\mathrm{N}$-terminus (dark blue). The globular C-terminal domains of Spc25 (green) and Spc24 (red) dimerize to form a "receptor" connecting to the Nsl-1 subunit of the Mis12 complex. The long coiled-coils contributed by each of the four proteins join together in the tetramerization domain (black box). The Hec1/Ndc80 subunit also contains an unstructured "hinge" region (dark blue) that permits complex flexibility in vitro. 


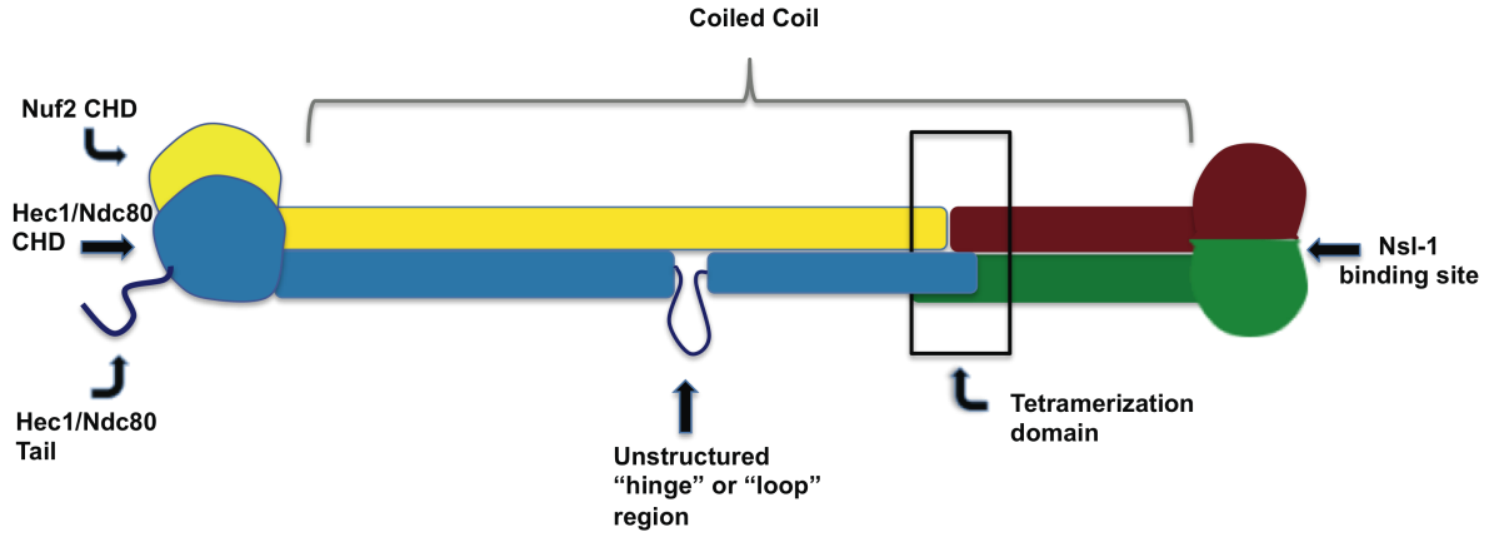

Figure 3-1 


\section{The Ndc80 complex as a kinetochore coupler}

A depolymerization-based coupler must use microtubule shortening to both power cargo movement and to provide directionality. A key to understanding how kinetochores act as couplers will be a careful description of how they engage microtubules. The Ndc80 complex is currently the best candidate for this elusive coupling activity. The Ndc80 complex directly binds microtubules in vitro with $\sim 0.5-3 \mu \mathrm{M}$ affinity (Cheeseman et al. 2006; Ciferri et al. 2008). When recombinant Ndc80 complex is attached to polystyrene beads, the coated beads are capable of binding the lateral sides of stabilized microtubules, remaining attached to the plus end of a microtubule in response to tension, and tracking with a depolymerizing microtubule (McIntosh et al., 2008; Powers et al., 2009). These data argue that $\mathrm{Ndc80}$ is a kinetochore coupler capable of moving a large cargo in vitro. In vivo, mutants of the Hec1/Ndc80 subunit generate kinetochores that are unable to productively bind microtubules, congress chromosomes to the metaphase plate or segregate them during anaphase (Guimaraes et al. 2008; Miller et al. 2008). Injection of a monoclonal antibody targeting Hec1/Ndc80 results in greater stretch between sister kinetochores, loss of K-fiber flux and the inability of kinetochores to release microtubules (DeLuca et al., 2006). Thus the in vivo data corroborate what is seen in vitro and point toward a direct role for the Ndc80 complex in depolymerization-coupled movement.

The unstructured tails of tubulin contribute significantly to the binding affinity of the Hec1/Ndc80 subunit (Ciferri et al. 2008; Miller et al. 2008). These negatively charged C-terminal tails (often referred to as E-hooks) interact with the Hec1/Ndc80 tail, which contains numerous positively charged residues. Electrostatic interactions between the two 
tails are thought to be a primary mode of Ndc80 complex binding. Cryo-EM images reveal that the Hec1/Ndc80 CHD contacts the microtubule directly, and mutations in residues which contact the microtubule greatly reduce binding affinity in vitro (Cheeseman et al. 2006; Ciferri et al. 2008; Wilson-Kubalek et al. 2008; Alushin et al. 2010). This suggests that the interaction between the Ndc80 complex and a microtubule is bipartite, and is generated by a combination of binding affinity from the CHD and the unstructured tail. The CHD also requires E-hooks for maximal binding (our unpublished data), suggesting a dual role for these unstructured tails of tubulin.

The cryo-EM structure of a chimeric version of the human complex has been solved to a resolution of $8.6 \AA$. This higher-resolution structure allowed the visualization of Ndc80 secondary structure and facilitated the unambiguous docking of the "Bonsai" crystal structure within the cryo-EM density (Alushin et al. 2010). An important finding from this study was the identification of a binding interface between the Ndc80 complex and a microtubule. The structure shows a small set of surface residues on the Hec1 CHD (referred to as the "toe") that bind a conserved surface spanning the $\alpha-\beta$ or $\beta-\alpha$ subunit interface on the lateral surface of a stabilized microtubule. Interestingly, we have characterized a point mutant in this "toe" region of the Hecl CHD, which renders kinetochores highly defective in assays for depolymerization-coupled movement and generation of productive microtubule attachments (Tooley et. al, 2011). Thus, the biochemistry and the genetics of the Ndc80 complex have converged to argue very strongly that the Ndc80 complex is a direct mediator of force production in human cells. It is difficult to identify the density of the E-hooks in the cryo-EM structures, but we have identified additional lysine residues in the Hec1 CHD that lie close to the exit point of the 
E-hooks (Alushin et al. 2010). These lysines also are essential for metaphase congression of chromosomes (Tooley et al., 2011), strongly arguing that E-hooks also interact with the Hec1 CHD to generate additional coupling forces.

In vitro binding experiments using recombinant $\mathrm{Ndc} 80$ complex have demonstrated a cooperative aspect to Ndc80 binding (Cheeseman et al. 2006; Ciferri et al. 2008; Alushin et al. 2010). Metazoan Ndc80 complex has a much higher affinity for another $\mathrm{Ndc} 80$ on a microtubule than a free microtubule, but such cooperativity is not seen in the budding yeast complex. The tail of $\mathrm{Hec} 1 / \mathrm{Ndc} 80$ is thought to be responsible for driving cooperativity, since in its absence cooperative binding is lost. Cooperativity could come from tail-tail interactions (since the Hec1 tail alone shows cooperative binding characteristics) (Miller et al. 2008) and/or from interactions with the negatively charged regions on adjacent $\mathrm{Ndc80}$ complexes (such as those that reside in the Ndc80/Hec1 or Nuf2 CHDs) (Ciferri et al. 2008). These interactions between dual CHDs of adjacent complexes may also be coordinated by the unstructured tail (Alushin et al. 2010). In vitro cross-linking and MS/MS studies have identified interactions between residues in the Hec1 tail and residues in both the Hec1 and Nuf2 CHDs (Maiolica et al., 2007). Furthermore, mutating seven serines in the Hec1 tail to aspartic acid (mimicking phosphorylation) diminishes Ndc80 complex cluster formation (Alushin et al. 2010). Thus the unstructured tail of metazoan Hec1/Ndc80 appears to have two functions, first to increase the affinity for tubulin and second to modulate cooperativity. These data argue for a model of depolymerization-based coupling where groups of Ndc80 work on a single tubulin protofilament. Current models suggest that rather than slide ahead of a depolymerizing end, the "toe" of the most proximal Ndc 80 complex releases in response 
to protofilament curvature. Following its release, the most attractive binding site for that Ndc80 complex would be behind the last subunit on the straight protofilament (Figure 32).

Despite convincing in vitro data, the in vivo contributions of cooperativity remain in question. The Hec1/Ndc80 tail is critical for kinetochore function in vertebrates but dispensable in budding yeast (Guimaraes et al., 2008; Kemmler et al., 2009; Miller et al., 2008). Moreover, diverse organisms build kinetochores containing roughly the same number of Ndc80 complexes per MT (6-9) (Joglekar et al., 2008; Joglekar et al., 2006; Johnston et al., 2010). Current published data make it unclear whether the essential vertebrate activity of the tail stems from its microtubule binding activities or the regulation of cooperative binding. We are currently characterizing a separation of function mutant that suggests that cooperativity is not absolutely required for congression of chromosomes to the metaphase plate (our unpublished data). We cannot rule out more subtle roles for cooperativity, such as enhancing rates of chromosome movement. Nevertheless, our data argue that the cooperativity model shown in Figure 3-2 is not an absolute requirement for chromosomes congression. 
Figure 3-2: Models for depolymerization-coupled movement involving Ndc80

A vertebrate kinetochore uses the Ndc80 complex to couple to depolymerizing microtubule through biased diffusion. The "toe" region of the $\mathrm{Hec} 1 / \mathrm{Ndc} 80 \mathrm{CHD}$ reacts to the conformational state of the microtubule lattice, binding to straight tubulin protofilaments between two subunits but becomes dislodged from depolymerizing tubulin subunits (adapted from Alushin, GM et al., 2010). Following displacement of the CHD toe, the Hec1/Ndc80 tail acts as a sliding tether allowing the kinetochore to retain partial connection to the microtubule through electrostatic interactions with the charged tubulin E-hooks (first arrow). The displaced Ndc80 complex then undergoes biased diffusion and rebinds at the next available tubulin subunit (second arrow) - a process that may be aided by cooperative binding of Ndc80 complexes. Budding yeast, in contrast, make use of a dual coupler involving both the Ndc80 complex and also the Dam1 ring complex to slide behind a depolymerizing microtubule. Here, the Dam1 complex would be able to ride the conformational wave created at the microtubule plus end during depolymerization. 


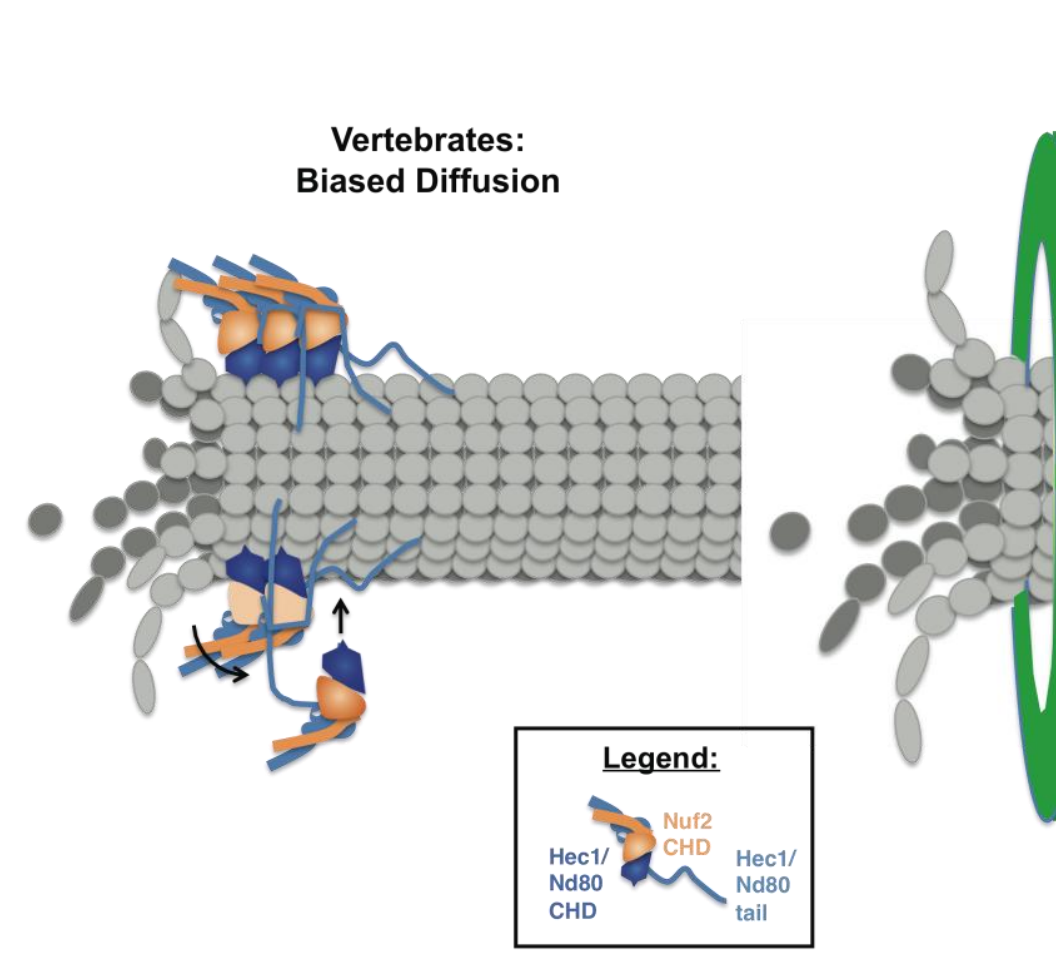

Budding yeast:

Sliding Clamp

Figure 3-2 


\section{Regulation of the Ndc80 complex}

The interaction between the Ndc80 complex and microtubules is highly regulated. The best-studied mode of Ndc80 regulation remains phosphorylation by the mitotic kinase Aurora B/Ipl1. The catalytic member of the four-member chromosomal passenger complex (CPC) (Ruchaud et al., 2007), Aurora B/Ipl1 phosphorylates Hec1/Ndc80 in vitro and in vivo on a number of sites contained in its $\mathrm{N}$-terminal unstructured tail (Akiyoshi et al., 2009; Cheeseman et al., 2002; Cheeseman et al., 2006; DeLuca et al., 2006; Guimaraes et al., 2008; Welburn et al., 2010). According to current models, phosphorylation of Hec1/Ndc80 occurs in response to the presence of an aberrant microtubule connection, such as a syntelic or merotelic attachment. As kinetochore interactions with a microtubule strengthen, kinetochores come under tension and the Ndc80 complex is stretched away from the inner centromere, where Aurora B resides (Liu et al. 2009; Welburn et al. 2010). In support of this, artificially tethering Aurora B kinase to the outer kinetochore causes kinetochores to be displaced from microtubules (Liu et al., 2009). As the interaction between the Hec1/Ndc80 tail and tubulin E-hooks is driven by charge (Miller et al. 2008), the addition of negatively charged phosphates to the highly basic region of $\mathrm{Hec} 1 / \mathrm{Ndc} 80$ (net charge of +10 in humans) is thought to disrupt electrostatic interactions and loosen microtubule attachments. Indeed, phosphorylation of the $\mathrm{Ndc} 80$ complex or the $\mathrm{N}$-terminal regions of $\mathrm{Hec} 1 / \mathrm{Ndc} 80$ causes a marked decrease in microtubule binding ability in vitro (Cheeseman et al. 2006; Ciferri et al. 2008; Welburn et al. 2010). In vivo, mutations to Aurora sites in the Hec1/Ndc80 tail cause either displacement from microtubules and loss of cell viability (serine to aspartic acid mutations) (Guimaraes et al. 2008; Welburn et al. 2010) or errors in chromosome 
congression (serine to alanine mutations) (Deluca et al. 2006; Welburn et al. 2010). Most studies examining tail regulation by Aurora B have largely utilized an all-or-none approach, whereby all tail sites are either altered or left alone. However, it remains possible that certain sites along the $\mathrm{Hec} 1 / \mathrm{Ndc} 80$ tail are more critical than others, or that sites are phosphorylated in a spatial or temporal fashion. One speculative model would be for the tail to exist in various states of phosphorylation: a fully phosphorylated tail would completely disengage from microtubules (error correction), while a partially phosphorylated tail would be capable of acting as a sliding tether but not a cooperativity factor (see below and Figure 3-3). It will be important for future studies to test models such as this by taking a more nuanced approach to examining Aurora regulation.

A second mechanism of Ndc80 regulation involves the mitotic kinase Nek2A. Nek2A phosphorylates Hec1/Ndc80 in vitro on a serine located in the CHD region (Chen et al., 2002; Du et al., 2008). Replacing this serine with an alanine leads to decreased MT binding in vitro and defects in chromosome congression in vivo. Conversely, mutating this serine to glutamic acid increases the affinity of Hec1 for microtubules in vitro (Du et al. 2008). This serine is located adjacent to a CHD "toe" lysine (K166) which in humans is highly important for microtubule binding both in vitro (Ciferri et al. 2008; Alushin et al. 2010) and in vivo (Tooley et al., 2011). Replacing that lysine with a glutamic acid severely reduces binding. It remains puzzling why the addition of a negative charge on neighboring amino acids yields such disparate effects on microtubule binding.

Regulation of the Ndc80 complex may be more elaborate than first envisioned. In the future, it will be important for studies to delineate the timing of individual regulatory steps. Such information will also need to be integrated with the physical shifts that the 
Ndc80 complex undergoes in the kinetochore in response to various stages of microtubule attachment (see below).

\section{A model for Ndc80 function}

Kinetochores need to bind microtubules tight enough to move chromosomes, but these attachments must be highly dynamic to allow flux when K-fiber microtubules treadmill towards the spindle poles (Joglekar et al., 2010). This requirement suggests that the microtubule attachment might act as a slip-clutch, which alternates between a tight binding and a slipping mode. Tight and weak binding could be generated by the regulation of cooperativity, although there is an apparent conflict for how a microtubule might flux if the Ndc80 complex only releases microtubules in response to curvature changes that accompany depolymerization. A classic theoretical model suggested that the attachment was generated through numerous low affinity interactions. The "Hill Sleeve" model envisioned a number of low-affinity microtubule binding activities residing in the kinetochore sleeve (Hill, 1985). These interactions could easily be broken and remade as the kinetochore moved along the surface of a microtubule. When a microtubule depolymerizes (and a set of attachment points are lost) there are still numerous other potential attachment sites. As long as the equilibrium favors rebinding along the surface of the microtubule over complete detachment, enough attachments could be maintained on a depolymerizing microtubule to generate force. The binding characteristics of the Ndc80 complex reflect aspects of both these models. In higher eukaryotes, we propose a model whereby the Hec1/Ndc80 tail converts between a sliding tether and a cooperativity factor. During initial stages of attachment, the tail functions as a tether (thereby generating "Hill sleeve binding" through numerous low-affinity interactions) allowing 
the kinetochore to work by biased diffusion and maintain contact with a microtubule during periods of depolymerization. As attachments mature, the tail may facilitate tighter binding by linking adjacent complexes along a microtubule protofilament. This conversion between low and high affinity would likely be regulated by the Aurora B kinase. As outlined above, cooperativity in unphosphorylated Hec1/Ndc80 would allow the CHD to bind a microtubule tightly but then release in response to microtubule depolymerization. In an Ndc80 complex where the tail is phosphorylated, the CHD may bind less tightly and act more like a slip-clutch.

It is important to note that in vitro the yeast Dam1 and vertebrate Ska1 complexes bind microtubules with a higher affinity than the Ndc80 complex and are also more processive than the Ndc80 complex in depolymerization-coupled movement assays (Asbury et al., 2006; Welburn et al., 2009). This has led to the assertion that these complexes, and not Ndc80, are the key couplers at the kinetochore. While the in vitro data are compelling (and it is likely that both complexes contribute to depolymerizationcoupled movement), we must emphasize that the Ndc80 complex is the only proposed coupler which is absolutely required for chromosome movements in vivo in all eukaryotes. We currently favor a model whereby budding yeast and vertebrate kinetochores use distinct mechanisms to couple to microtubules. This subject will be addressed later in this review. 
Figure 3-3: Model for differential Hec1/Ndc80 tail phosphorylation

Proposed model whereby differential phosphorylation states confer distinct functions to the $\mathrm{Hec} 1 / \mathrm{Ndc} 80$ unstructured tail. Left, during initial binding reactions the $\mathrm{Hec} 1 / \mathrm{Ndc} 80$ tail is partially phosphorylated by the Aurora B kinase - creating a situation where the tail is competent to interact with tubulin E-hooks but is deficient in cooperative binding (Tail Tethering Mode). Following initial interactions with microtubules and an increase in tension applied to kinetochores (below right), the tail is moved away from Aurora B. This, along with recruitment of PP1 to kinetochores, results in complete tail dephosphorylation and allows formation of cooperative interactions between $\mathrm{Ndc} 80$ complexes. During error correction (top right), the tail becomes highly phosphorylated and can neither tether $\mathrm{Ndc} 80$ to microtubules nor drive cooperativity. 


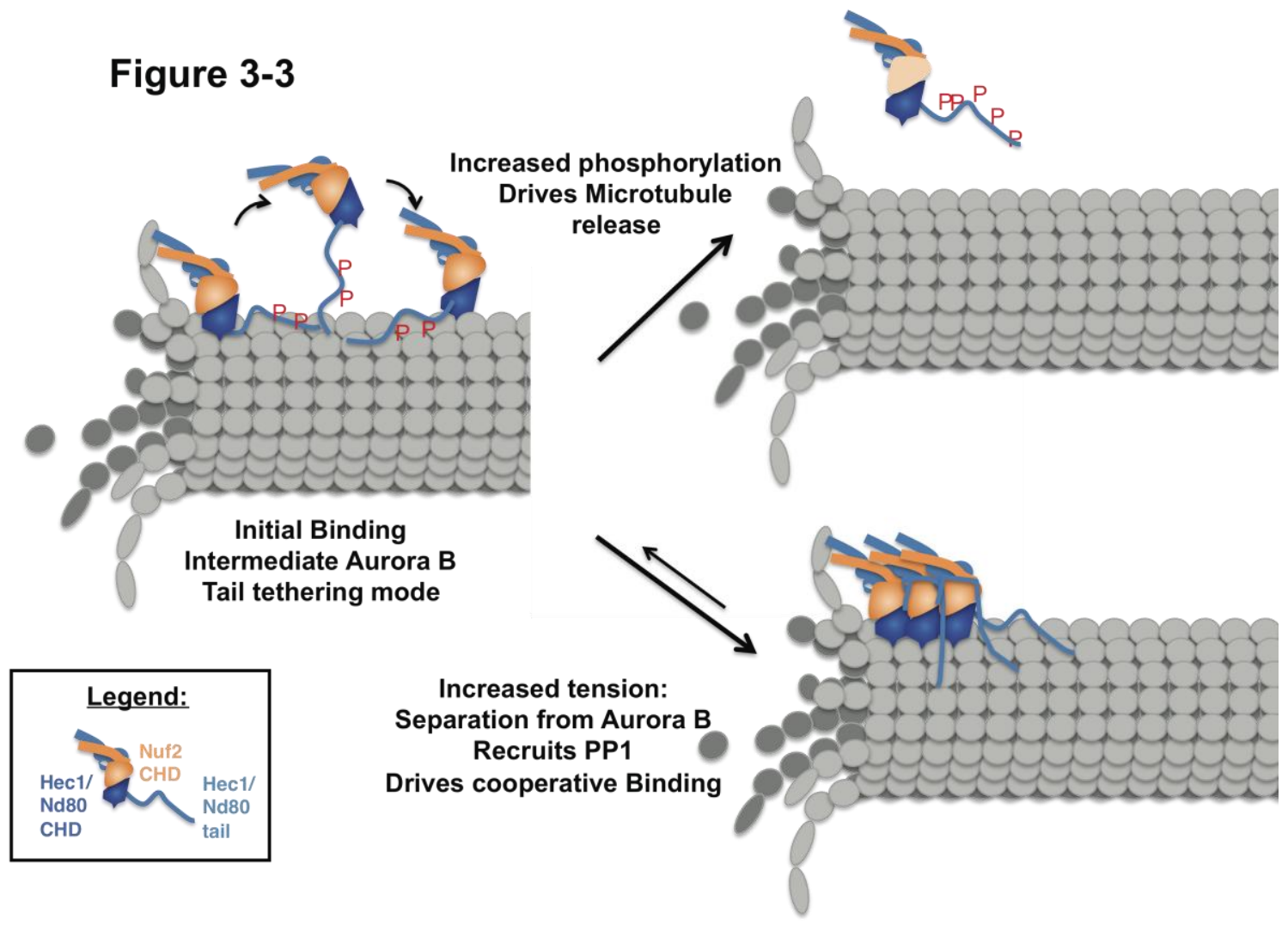




\section{Ndc80 complex as an integrator of kinetochore function}

The conserved kinetochore parts list is likely nearing completion, and tremendous progress has been made to define the physical connections and dependencies that exist during kinetochore assembly (Cheeseman and Desai, 2008; Gascoigne and Cheeseman, 2011). However, we are only beginning to fully appreciate how dynamic this large kinetochore structure can be. A great challenge moving forward will be to tease apart why the vertebrate kinetochore relies on more than 100 proteins to facilitate accurate chromosome segregation, and how microtubule binding is linked to error correction and checkpoint signaling. The Ndc80 complex has been implicated in all three aspects of kinetochore function, but it clearly does not act alone. Therefore, we must better understand how this microtubule coupler operates within the ever-changing kinetochore microenvironment.

Within the kinetochore the Ndc80 complex exists as part of a larger supercomplex, often referred to as the KMN network (Cheeseman et al., 2006; Kline-Smith et al., 2005). Consisting of the four-member Ndc80 complex, the four-member Mis12 complex, KNL-1 and Zwint (in most species), this conserved network serves as the core microtubule attachment site at the kinetochore. $\mathrm{KMN}$ is brought to centromeres via links with CENP-A and CENP-C (Santaguida and Musacchio, 2009). The Ndc80 complex is integrated into KMN by binding the Nsl-1 subunit of the Mis12 complex (Petrovic et al. 2010). It has also been shown to depend on KNL-1 and the CENP/H///K complex for kinetochore recruitment, though differences exist between species (Cheeseman et al., 2008; Desai et al., 2003; Okada et al., 2006). Members of the KMN complex, in turn, serve as a platform for recruiting additional outer kinetochore components. The Ndc80 
complex is required for kinetochore localization of the Ska and RanGAP/RanBP2 complexes, both of which are implicated in chromosome congression and checkpoint signaling (Hanisch et al., 2006; Joseph et al., 2004; Ohta et al., 2010). It is also required to bring the checkpoint proteins Mad1, Mad2 and MPS1 to the kinetochore (DeLuca et al., 2003; Hori et al., 2003; Martin-Lluesma et al., 2002; McCleland et al., 2003; Meraldi et al., 2004). (Notably, no physical interactions have been detected between any of these proteins and the Ndc80 complex). KNL-1 is responsible for bringing a separate set of proteins to the kinetochore. These proteins include Zwint, RZZ, Spindly, Dynein, Bub1, Bub3, BubR1 and PP1 (Desai et al., 2003; Gassmann et al., 2008; Kiyomitsu et al., 2007; Liu et al., 2010). The Mis12 complex, the final member of KMN, serves as the bridge linking Ndc80 and KNL-1 (Petrovic et al. 2010). Therefore, the outer kinetochore which emanates from the KMN network can be thought of as containing two "branches" - one assembled by KNL-1 and the other by the Ndc80 complex (Wan et al. 2009; Maresca and Salmon 2010) (Figure 3-4).

Recent work has demonstrated that the two branches are not static. A compelling study by the Salmon lab and colleagues utilized high-resolution single molecule imaging to generate a map that pinpoints the location of many proteins within the kinetochore. Importantly, this map also captures positional changes that occur in response to the loss of tension applied on kinetochores by microtubules (Wan et al., 2009). Following loss of tension, the Ndc80 complex moves backward in the kinetochore (i.e. closer to CENP-A). KNL-1 does not move nearly as far back as Ndc80 - resulting in Ndc80's position relative to KNL-1 being altered (Figure 3-4). This shift may be a consequence of rearrangements that occur between Mis12 complex members following loss of tension. 
Alternatively, it may indicate more centromere-proximal shifts involving the CENP-C and CENP-I proteins. Regardless, such changes demonstrate a dynamic rearrangement of the kinetochore architecture following MT engagement, an observation first made by electron tomography (Dong et al., 2007). It seems plausible to suggest that in addition to KNL-1, the N-terminus of Hec1/Ndc80 also is being shifted in relation to RZZ and PP1, two proposed regulators of the $\mathrm{Ndc} 80$ complex that are recruited to this region of the kinetochore (Gassmann et al. 2008; Gassmann et al. 2010; Liu et al. 2010). Further experiments could test this prediction, and could also overlay additional proteins (such as the Ska complex) onto this map.

Another pair of elegant studies extend this concept by linking shifts in kinetochore architecture to SAC signaling (Maresca and Salmon, 2009; Uchida et al., 2009). The authors here uncover evidence of intra-kinetochore stretching forces within a single kinetochore, and they present a strong argument that intra-kinetochore alterations rather than the separation of sister kinetochores - is the primary readout driving SAC signaling. The authors correlate intra-kinetochore stretch with SAC signaling (low stretch, high SAC signal and high stretch, loss of SAC signal). Remarkably, they can produce the low SAC signal even when inter-KT stretch (the separation of sister centromeres) was reduced but intra-KT stretch remained elevated.

We are now beginning to better understand how the Ndc80 complex operates within the kinetochore. Based on these studies, the rough outlines can now be drawn for a model integrating microtubule engagement with checkpoint signaling. Within the outer kinetochore, there are two branches emanating from the Mis12 complex (Wan et al. 2009). The branch built by Ndc80 contains a number of factors implicated in microtubule 
binding, chromosome congression and SAC signaling. The KNL-1 branch contains numerous checkpoint proteins but also factors which can influence the binding of Ndc80 to microtubules. Following kinetochore engagement with a microtubule, a shift occurs between the two branches. This shift has the effect of moving MT binders, binding regulators and signaling modules relative to one another (Figure 3-4). Such dynamic movements may also hint at multivalent docking sites for proteins such as Mad1/Mad2 and the Ska complex at the kinetochore - and could explain the inability to detect a direct interaction between the Ndc80 complex and any of the proteins it helps recruit (Maresca and Salmon, 2010). Much of the details remain to be worked out, but in this dynamic view of the kinetochore the Ndc80 complex emerges not only as a microtubule coupler but also as a key integrator of kinetochore signaling. 
Figure 3-4: The vertebrate Ndc80 complex integrated into the overall kinetochore architecture

(A) Prior to microtubule attachment, the overall vertebrate kinetochore architecture is less structured and possesses a number of factors involved in the initial capture of microtubules. Here, Ndc80 complex binding is initially inhibited by the RZZ complex, and the $\mathrm{N}$-terminus of KNL-1 lies in close proximity to the N-terminus of the Ndc80 complex. (B) Following microtubule capture the kinetochore becomes templated by the microtubule and a number of tension-dependent shifts occur among kinetochore proteins. (C) During periods of microtubule depolymerization CENP $\mathrm{H} / \mathrm{I} / \mathrm{K}$ binding is diminished and Ndc80 now tracks near the end of the shortening microtubule. 

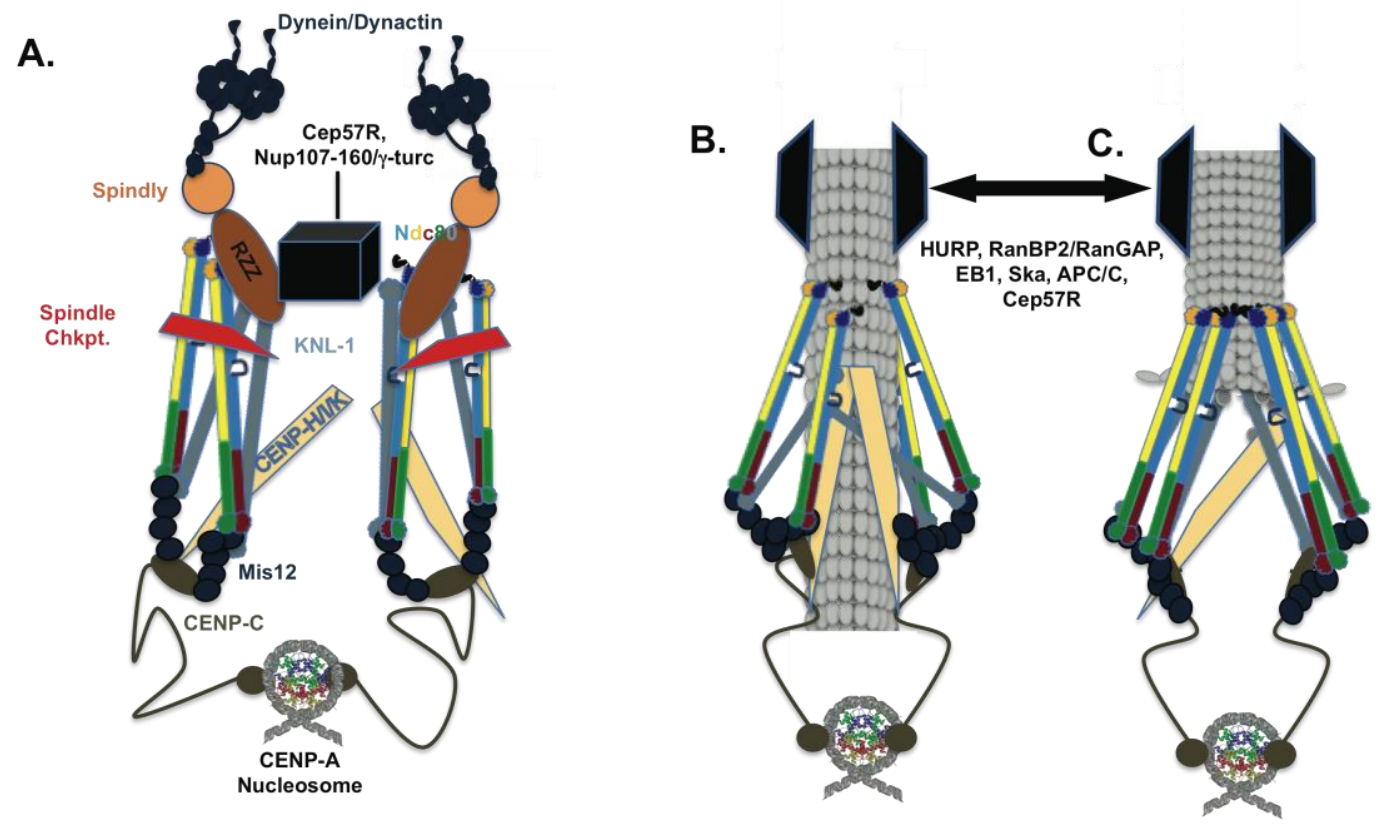

Figure 3-4 


\section{Ndc80 in budding yeast vs. Hec1 in humans}

Despite its conserved presence in eukaryotic kinetochores, it remains unclear whether the Ndc80 complex has been used in the same way as species evolve. Data used to build models for Ndc80 function are often pooled from a number of divergent organisms, namely budding yeast, worms and humans. However, kinetochore proteins are known to have evolved quite rapidly (Meraldi et al., 2006), and contradictions about Ndc80 function persist. Therefore, it is important to more carefully discern which functional elements of the Ndc80 complex have been conserved and which have evolved to meet the needs of a particular kinetochore structure. The most striking example of functional divergence involves the unstructured tail of Hec1/Ndc80. In vitro, the tail is required to enhance the MT binding of both the yeast and human complexes (although only the metazoan complexes have been shown to exhibit cooperative binding).

Additionally, recombinant yeast and human Ndc80 complex can both couple microtubule depolymerization to bead movement (Wei et al. 2007; Ciferri et al. 2008; Powers et al. 2009). However, the story is much different in vivo. While the tail is absolutely critical for kinetochore function in humans (Guimaraes et al. 2008; Miller et al. 2008), it appears dispensable in budding yeast (Kemmler et al. 2009, D. Burke unpublished data). A likely explanation for such a discrepancy is the presence of the Dam1 complex in budding yeast. The Dam1 complex can form rings around MTs in vitro and can slide along a MT in response to force (Asbury et al., 2006; Westermann et al., 2005; Westermann et al., 2006). When bound to beads it is capable of moving cargo along a depolymerizing MT in a processive manner (Asbury et al., 2006; Grishchuk et al., 2008a). Furthermore, free Dam1 complex flowed into an Ndc80 bead-based experiment can confer increased MT 
binding and processivity to the yeast Ndc80 complex (Lampert et al.; Tien et al., 2010). Therefore, in budding yeast the tail of Ndc80 may be less important because Dam1 also functions as a key kinetochore coupler. Here, the kinetochore works by a sliding mechanism, riding the conformational wave caused by a depolymerizing MT (Asbury et al., 2011). In contrast, the metazoan complex acts as a biased diffuser built upon low affinity charge-based interactions, using the tail and cooperative CHD binding together with conformation-dependent microtubule release (Figures 3-2 and 3-3). An intriguing evolutionary intermediate may exist in fission yeast, where the Dam1 complex is present at kinetochores in lower amounts (Joglekar et al. 2008) and is not essential for viability (Liu et al., 2005; Sanchez-Perez et al., 2005). In this organism, however, the Dam1 complex is required to retrieve unclustered kinetochores (Franco et al., 2007). It would be interesting to know the requirements of the Ndc80 tail in this organism.

The question of whether a Dam1-like complex exists in vertebrates has been the subject of much study and debate. In humans the Ska complex has emerged as a leading candidate for the functional counterpart of the Dam1 complex, but its case is not airtight. While capable of binding MTs and moving a bead cargo (Welburn et al. 2009), it has not been demonstrated that the Ska complex can enhance Ndc80 tracking abilities in a beadbased coupling assay. Furthermore, an emerging body of data points to a prominent role for the Ska complex in kinetochore recruitment of the APC/C and checkpoint signaling, a characteristic that has not been ascribed to Dam1 (Daum et al., 2009; Hanisch et al., 2006; Ohta et al., 2010; Theis et al., 2009).

The discrepancy over Hec1/Ndc80 tail function raises the question of whether other functional elements of Ndc80 may have evolved. There are potential points of 
divergence. For example, the mechanism of Aurora B/Ipl1-mediated error correction may also differ between species. In humans, the unstructured tail of Hec1 contains numerous Aurora B target sites and mutation of these sites creates significant errors in microtubule binding both in vitro and in vivo. In budding yeast, mutation of Ipl1 sites - while creating binding defects in vitro (Cheeseman et al. 2006) - does not appear to be as consequential in vivo (Akiyoshi et al. 2009). Ipl1 also targets numerous sites on the Dam1 complex, including a set of sites on Dam1 that disrupt the interaction between the Dam1 and Ndc80 complexes (and presumably Dam1 complex KT recruitment) (Shang et al. 2003; Tien et al. 2010). As mutation of these Dam1 sites creates a strong phenotype in vivo (Shang et al., 2003), it is possible that this is a more prominent means of attachment turnover in budding yeast. (Notably, Aurora B phosphorylation of the Ska complex has not been reported). Furthermore, while MPS1 phosphorylation of the Ndc80 tail also is important in budding yeast, it is unclear whether this event is conserved across species (Kemmler et al. 2009).

A number of proteins localize to kinetochores and spindles in vertebrates but not in budding yeast (Figures 3-4 and 3-5). These include members of the RanBP2/RanGAP complex, the Nup107-160 complex (Mishra et al., 2010; Zuccolo et al., 2007), RZZ, Spindly, Dynein, HURP (Koffa et al., 2006; Sillje et al., 2006; Wong and Fang, 2006) and Cep57R (Emanuele and Stukenberg 2007). While analogous proteins may yet to be identified in budding yeast (Pagliuca et al., 2009) for now these proteins constitute a black box at the vertebrate kinetochore where relatively little is known about the precise contributions made by these factors. As many of these proteins have been implicated in 
aspects of chromosome congression, there is still much work to be done to understand how kinetochores couple microtubule depolymerization to chromosome movement. 
Figure 3-5: The budding yeast Ndc80 complex integrated into the overall kinetochore architecture

(A, B, C) In budding yeast many of the conserved factors remain; however, factors involved in lateral attachment are distinct and the Dam1 complex plays a primary role (through interactions with the Ndc80 complex) in coupling to a depolymerizing microtubule. 
Figure 3-5
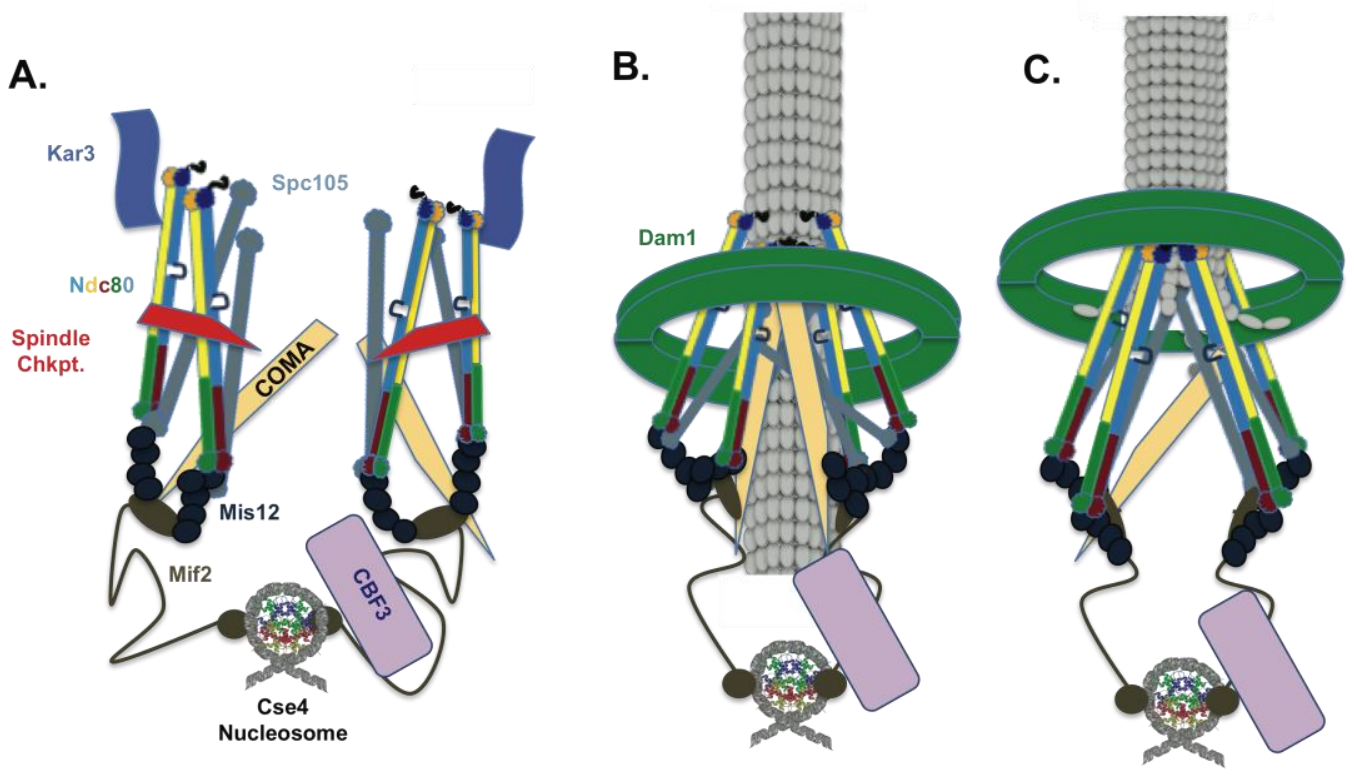


\section{Chapter 4: \\ THE NDC80 COMPLEX USES A TRIPARTITE ATTACHMENT POINT TO COUPLE MICROTUBULE DEPOLYMERIZATION TO CHROMOSOME MOVEMENT}

This chapter is based on the following publication:

Tooley, JG, Miller, SA and Stukenberg, PT. "The Ndc80 complex uses a tripartite attachment point to couple microtubule depolymerization to chromosome movement" Molecular Biology of the Cell. (2011) 22, 1217-1226. 
In kinetochores, the $\mathrm{Ndc} 80$ complex couples the energy in a depolymerizing microtubule to perform the work of moving chromosomes. The complex directly binds microtubules using an unstructured positively charged $\mathrm{N}$-terminal tail located on Hec1/Ndc80. Hec1/Ndc80 also contains a calponin homology domain (CHD) that increases its affinity for microtubules in vitro, yet whether it is required in cells and how the tail and CHD work together are critical unanswered questions. Human kinetochores containing Hec1/Ndc80 with point mutations in the CHD fail to align chromosomes or form productive microtubule attachments. Kinetochore architecture and spindle checkpoint protein recruitment are unaffected in these mutants, and the loss of CHD function cannot be rescued by removing Aurora B sites from the tail. The interaction between the Hec1/Ndc $80 \mathrm{CHD}$ and a microtubule is facilitated by positively charged amino acids on two separate regions of the CHD and both are required for kinetochores to make stable attachments to microtubules. Chromosome congression in cells also requires positive charge on the Hec1 tail to facilitate microtubule contact. In vitro binding data suggest that charge on the tail regulates attachment by directly increasing microtubule affinity as well as driving cooperative binding of the CHD. These data argue that in vertebrates there is a tripartite attachment point facilitating the interaction between Hec1/Ndc80 and microtubules. We discuss how such a complex microtubule-binding interface may facilitate the coupling of depolymerization to chromosome movement. 


\section{Introduction}

The kinetochore is a macro-molecular machine assembled on centromeric chromatin during mitosis. Its predominant role is to anchor replicated DNA to spindle microtubules and to couple the energy of microtubule depolymerization to segregate sister chromatids. The kinetochore also regulates the dynamics of captured microtubules, corrects errors in microtubule attachment, and generates the spindle checkpoint, a failsafe mechanism used by the cell to ensure that all chromosomes are properly attached to the mitotic spindle (Cleveland et al., 2003; Santaguida and Musacchio, 2009).

The mammalian kinetochore is comprised of more than 80 proteins, many of which are evolutionarily conserved (Cheeseman and Desai, 2008). The four-member Ndc80 complex, consisting of Ndc80 (Hec1 in humans), Nuf2, Spc24 and Spc25, is critical for kinetochore function (Wigge and Kilmartin, 2001; Deluca et al., 2002; Martin-Lluesma et al., 2002; Deluca et al., 2003; McCleland et al., 2003; McCleland et al., 2004). At the kinetochore, the Ndc 80 complex is closely associated with KNL-1 and the four-member Mis12 complex to form the "KMN network." This KMN super-complex serves as the major attachment site for captured spindle microtubules (Cheeseman et al., 2006). In all tested eukaryotic systems the Ndc80 complex directly binds microtubules and loss of its activity results in failure by the kinetochore to bind microtubules and to congress chromosomes to form a metaphase plate (Cheeseman and Desai, 2008). In addition, mutant kinetochores cannot align chromosomes when they lack the unstructured tail of Hec1/Ndc80, which is critical for the complex to bind microtubules (Wei et al., 2007; Ciferri et al., 2008; Guimaraes et al., 2008; Miller et al., 2008). The Ndc80 complex can track on the plus end of a depolymerizing microtubule in vitro and can 
produce force while doing so (Powers et al., 2009). Together these data argue that the Ndc80 complex directly binds microtubules to allow kinetochores to move chromosomes.

The $\mathrm{N}$-terminus of the $\mathrm{Hec} 1 / \mathrm{Ndc} 80$ protein binds microtubules and contains a calponin homology domain (CHD) in addition to the 80 amino acid unstructured tail (Wei et al., 2007). The remainder of Hec1/Ndc80 forms a 500A coiled coil that dimerizes with Nuf2. Like Hec1/Ndc80, Nuf2 possesses a CHD at its N-terminus (Ciferri et al., 2008). The C-termini of Hec1/Ndc80 and Nuf2 are joined to the $\mathrm{N}$-terminal coils of Spc24 and Spc25 through a tetramerization domain (Maiolica et al., 2007). In contrast to the more distal Hec1/Ndc80 and Nuf2, which point outward from the DNA, the globular C-terminal regions of Spc24 and Spc25 are oriented closer to the centromere (Wei et al., 2005; Deluca et al., 2006; Wei et al., 2006; Wan et al., 2009).

To appreciate how kinetochores couple microtubule depolymerization energy to perform the work of moving chromosomes, it is critical to understand how the Ndc80 complex interacts with microtubules. Proteins often use a dual CHD to attach to actin filaments (Sjoblom et al., 2008) and a dual CHD is essential for microtubule interactions by the plus-end tracking protein EB1/Bim1 (Hayashi and Ikura, 2003; Slep and Vale, 2007; Zimniak et al., 2009). Similarly, the Hec1/Ndc80 CHD is incapable of binding microtubules on its own (Miller et al., 2008) and must exist in the context of the Ndc80 complex for binding to occur. Both Hec1/Ndc80 and Nuf2 CHD mutants decrease the affinity of the complex for microtubules in vitro, but this binding defect is less severe than in mutants lacking the unstructured tail (Ciferri et al., 2008). Cryo-EM images of recombinant $\mathrm{Ndc} 80$ complex bound to a microtubule reveal that a region of the Hec1/Ndc80 CHD - termed the "toe" - binds the surface of a microtubule at a discrete 
point between adjacent tubulin subunits (Alushin et al., 2010). In addition the Ndc80 complex has lower affinity for curved protofilaments compared to the lateral surface of microtubules. This suggests that the toe acts as a tubulin conformation sensor - allowing the Ndc80 complex to bind straight microtubules but causing release in response to protofilament curvature (Alushin et al., 2010). Importantly, the role of the Hec1/Ndc80 CHD in vivo remains untested.

The unstructured tail of $\mathrm{Hec} 1 / \mathrm{Ndc} 80$ is essential for kinetochore function in vertebrates (Guimaraes et al., 2008; Miller et al., 2008) and it has been proposed to have two functions. It can bind microtubules on its own in vitro and it increases the affinity of the dual CHD binding (Ciferri et al., 2008; Miller et al., 2008). The tail interaction requires the unstructured and negatively charged E-hooks on tubulin (Miller et al., 2008). The Hec1/Ndc80 tail is highly positively charged and phosphorylation of this motif by the Aurora kinases can decrease microtubule affinity (Cheeseman et al., 2006; Ciferri et al., 2008; Guimaraes et al., 2008). In addition, the tail of Hec1/Ndc80 has been shown to facilitate cooperative microtubule binding of the Ndc80 complex in vitro (Cheeseman et al., 2006; Ciferri et al., 2008; Alushin et al., 2010), and electron density that is presumed to be the tail residues facilitates tight packing of adjacent $\mathrm{Ndc} 80$ subunits along the surface of a microtubule. This tight packing along protofilaments suggests a model whereby tight affinity of the Ndc80 complex to microtubules would be generated by cooperative binding and that this could be released through the conformational changes that accompany depolymerization (Alushin et al., 2010).

In this study, we utilize a knockdown and rescue system in human cells to examine the in vivo contribution of the Hec1/Ndc80 CHD and the unstructured tail to 
binding microtubules and congressing chromosomes. We demonstrate that mutants lacking positive charge in either the CHD or the unstructured tail severely compromise kinetochore function. Our data suggest the Ndc80 complex couples microtubule depolymerization to chromosome movement using two microtubule-binding surfaces on the CHD which accurately positions $\mathrm{Ndc} 80$ on a microtubule. Furthermore, the vertebrate Ndc80 complex relies on an unstructured tail to strengthen binding through charge-based interactions. Our data demonstrate the critical requirement of the toe region of the Hec1/Ndc80 CHD, consistent with this being a conformational sensor. Our data also suggest that the E-hooks of tubulin interact with both the CHD and the Hec1/Ndc80 tails to increase affinity for a microtubule through electrostatic interactions. 


\section{Materials and Methods}

\section{Cloning of Hec1 constructs}

To facilitate cloning of mutants, the pGEX6P-2rbs WT human Ndc80 ${ }^{\text {bonsai }}$ construct (a gift from A. Musacchio) was modified by placing an Fse1 restriction site prior to the Nterminus of the $\mathrm{Hec} 1 / \mathrm{Spc} 25$ fusion. Ndc80 ${ }^{\text {bonsai }} \mathrm{CHD}$ mutants were generated using twostep PCR mutagenesis. The charge neutral tail mutant was generated by removing the WT tail and inserting a duplexed oligo coding for the Hec1/Ndc80 tail that contained the following 10 residues mutated to alanine: K2, R3, R13, K35, K42, K47, K53, K59, R60, and R67. For cell culture experiments, the first 84 amino acids of WT-Hec1 were cut from the Ndc80 ${ }^{\text {bonsai }}$ using Fse1 and PpuM1 and sub-cloned into the FLAG-Hec1 rescue vector (Miller et al., 2008) at these restriction sites. For CHD rescue experiments, WTHec1 containing the Fse1 restriction site was moved from the FLAG rescue vector into the pEGFP-N1 vector by using the Fse1 and Pst1 restriction sites. Mutants were cloned into EGFP rescue vectors either by moving them from the $\mathrm{Ndc} 80^{\text {bonsai }}$ construct through FLAG-Hec1 into pEGFP-N1 using the restriction sites listed above or by mutating the WT Hec1-EGFP construct using two-step PCR mutagenesis. For the Hec1 ${ }^{9 \mathrm{~A}}$ and Hec $1^{9 \mathrm{~A} / \mathrm{CHD} \text { mutant }}$ constructs, the following residues in the Hec1/Ndc80 tail were mutated to alanine: S4, S5, S8, S15, S44, T49, S55, S62, and S69. All constructs generated were verified by sequencing.

\section{Recombinant Protein Expression and Purification}

To create the Hec $1^{\mathrm{NEU}}$ and Hec1 ${ }^{\mathrm{K} 89 \mathrm{E} / \mathrm{K} 115 \mathrm{E}}$ mutants, the bi-cistronic plasmid that expresses human $\mathrm{Ndc} 80^{\text {bonsai }}$ was modified to fuse a 6-His tag to the N-terminus of Hec1. 6-His Ndc $80^{\text {bonsai }}$ was expressed, GST-purified, and cleaved with Precission Protease as 
described (Ciferri et al., 2008). Cleaved recombinant protein was further purified using Ni-NTA agarose (Qiagen). Hec ${ }^{\mathrm{WT}}, \mathrm{Hec}^{\Delta \mathrm{N}}$ and $\mathrm{Hec} 1^{\mathrm{K} 166 \mathrm{E}}$ lack the 6-His tag and were purified as described (Ciferri et al., 2008). Purified protein was dialyzed using a Slide-aLyzer Dialysis Unit (Thermo Fisher Scientific) into dilution buffer (100mM KCl, 10mM Na-HEPES, 1mM EDTA, 1mM DTT, $1 \mathrm{mM} \mathrm{MgCl} 2,0.1 \mathrm{M} \mathrm{CaCl}_{2}, 10 \%$ glycerol). Gel filtration was performed on a Superdex 200 column (GE Healthsciences).

\section{Microtubule Polymerization}

Phosphocellulose (PC)-purified bovine tubulin was polymerized to microtubules using taxol as previously described (Desai et al., 1999a). Microtubules were resuspended in dilution buffer supplemented with an equimolar concentration of taxol (20-40 $\mu \mathrm{M})$.

\section{Western Blotting and microtubule cosedimentation assays}

For determining microtubule binding and Hec1/Ndc80 knockdown levels, western blotting was performed as previously described (McCleland et al., 2004). For Hec1/Ndc80 knockdown, protein levels were determined by using anti-Hec1/Ndc80 9G3 antibody (GeneTex) at 1:2000. Cosedimentation assays were performed using taxolstabilized microtubules in dilution buffer and $20 \mu \mathrm{M}$ taxol as previously described (Cheeseman et al., 2006). Protein was detected using anti-hSpc25 (1:5000) (McCleland et al., 2004) and anti-tubulin (1:2000; DM1A, Sigma). The hSpc25 antibody was chosen for its intensity and linearity. HRP signal was detected with a FluoroChem FC2 imager (Alpha Innotech) and the signal intensity of each lane was measured using densitometry with FC2 software. Percent Ndc $80^{\text {bonsai }}$ bound was determined by dividing the signal in the pellet sample by the $100 \%$ input control. GraphPad Prism software was used to 
determine if the binding data was best represented by a fitted curve with or without a Hill coefficient (h) by performing an extra sum-of-squares F test.

\section{Circular dichroism measurements}

CD spectra of Hec1 $1^{\mathrm{WT}}$, $\mathrm{Hec}^{\Delta \mathrm{N}}$ and $\mathrm{Hec} 1^{\mathrm{K} 89 \mathrm{E} / \mathrm{K} 115 \mathrm{E}} \mathrm{Ndc} 80^{\text {bonsai }}$ proteins were collected in $10 \mathrm{mM}$ Tris buffer, $100 \mathrm{mM} \mathrm{NaCl}, 1 \mathrm{mM}$ EDTA, 5\% glycerol, pH 7.0 as previously described (Derewenda et al., 2007).

\section{Knockdown and Rescue of Hec1/Ndc80 in HeLa cells}

HeLa cells (ATCC) were maintained in Dulbecco's modified Eagle's medium (Invitrogen) supplemented with $10 \%$ fetal bovine serum (Invitrogen) in a humidified incubator at $37^{\circ} \mathrm{C}$ with $5 \% \mathrm{CO}_{2}$. For synchronization, cells were seeded in media containing $2 \mathrm{mM}$ thymidine for $24 \mathrm{hr}$, released into fresh media for $12 \mathrm{hr}$, arrested again in $2 \mathrm{mM}$ thymidine for $12 \mathrm{hr}$, released for 8-12 hr, and fixed for immunofluorescence. Hec1/Ndc80 siRNA sequence and rescue sequence modifications were used as previously described (Miller et al., 2008); however, CHD rescue constructs were expressed in the pEGFP-N1 vector. For knockdown and replacement experiments, cells were cotransfected at the first thymidine release with siRNA oligos and rescue plasmid using Lipofectamine 2000 (Invitrogen). Cells were transfected a second time with siRNA oligos at the second thymidine block using RNAiMax (Invitrogen). For mock and siRNA only controls, an empty pEGFP vector was used as the rescue plasmid. For CHD experiments, GAPD siRNA oligos (Thermo Scientific) were included as mock controls.

\section{Immunofluorescence}


Coverslips were co-fixed and extracted in PHEM buffer containing 2\% paraformaldehyde and $0.5 \%$ Triton X-100 for $20 \mathrm{~min}$ at room temperature. For cold lysis, cells were incubated on ice for 10-15 min in ice-cold media before fixation with paraformaldehyde. Antibodies used were anti-Hec1/Ndc80 9G3 (1:500), anti-Spc25 (1:500) (McCleland et al., 2004), anti-CENP-E (1:1000, a gift from Timothy Yen), anti-KNL-1 (1:1000, a gift from Arshad Desai), anti-Mad2 (1:100, a gift from Gary Gorbsky), anti-BubR1 (1:500, BD Biosciences), anti-ACA (1:500, Antibodies Incorporated), anti-tubulin (1:2000, DM1A, NeoMarkers) and FITC conjugated anti-tubulin (1:500; DM1A, Sigma). DAPI staining (1:5000 of a $5 \mathrm{mg} / \mathrm{ml}$ stock) was used to visualize DNA. Images in Supplemental Figures 3, 5 and 6 were captured by spinning disk confocal microscopy using either a $63 \mathrm{x}$ 1.4 NA or a 100x 1.4 NA Plan-Apochromatic Zeiss objective lens. A Zeiss Axiovert 200 inverted microscope was used with a Perkin Elmer confocal attachment and a krypton/argon laser with AOTF control for detecting at 488, 568, and $647 \mathrm{~nm}$. Digital images were obtained with a Hamamatsu digital CCD camera. Image acquisition, shutters, and z slices were all controlled with UltraView RS imaging software (Perkin Elmer). For Figures 1, 2, 3 and 4, images were collected using a 100x lens on a Deltavision microscope (Applied Precision) and deconvolved z-projections are shown. Inter-kinetochore distance measurements and relative Hec1/Ndc80 intensity levels were quantified using softWoRX imaging software.

\section{Hec1/Ndc80 Intensity Levels}

Hec1/Ndc80 9G3 antibody was used to stain cells in the $568 \mathrm{~nm}$ channel to allow comparison across mock, Hec1 ${ }^{\mathrm{WT}}$ and $\mathrm{Hec} 1^{\mathrm{K} 89 \mathrm{E} / \mathrm{K} 166 \mathrm{E}}$ conditions. Hec1/Ndc80 intensity was quantified by calculating kinetochore fluorescence in a square 9x9 pixel area and 
subtracting out background from surrounding $13 \times 13$ pixel area as previously described

(Hoffman et al., 2001). 


\section{Results}

\section{Cells expressing Hec1/Ndc80 CHD mutants assemble kinetochores properly}

We measured the functions of three lysines, (K89, K115, K166), which reside in the highly basic region of the Hec1/Ndc 80 CHD. Lysine 166 is part of the "toe" region of $\mathrm{Hec} 1 / \mathrm{Ndc} 80$ that docks near the tubulin dimer interface, and is proposed to be part of a microtubule conformation sensor (Alushin et al., 2010). Lysines 89 and 115 reside close together but away from the Hec1/Ndc 80 "toe". These amino acids are located close to the predicted exit point of negatively charged tubulin E-hooks (Figure 4-1B) (Alushin et al., 2010). Each lysine was first mutated to negatively charged glutamic acid, which are mutations that have been previously shown to create microtubule binding defects in vitro (Ciferri et al., 2008). We examined the function of each of these lysines individually as well as mutated in pairs (K89/K115 and K89/K166) (Figure 4-1C). Before testing mutants in vivo we confirmed that these point mutants do not grossly misfold the CHD in vitro. The study of Ndc80 function in vitro has been greatly enhanced by the generation of Ndc $80^{\text {bonsai }}$ constructs, which are engineered to lack the coiled coil domains (Ciferri $e t$ al., 2008). A subset of the mutations were engineered into $\mathrm{Ndc} 80^{\text {bonsai }}$ constructs, purified from E. coli and their circular dichroism (CD) spectrums and gel filtration profiles were compared to wild type Ndc $80^{\text {bonsai }}$. CHD mutants yielded CD spectra or fractionation patterns similar to control $\mathrm{Ndc} 80^{\text {bonsai }}$ complexes arguing that the mutations do not greatly change the overall structure of the proteins (Supplemental Figure 4-1A and 4-1B).

To ask if Hec1/Ndc80 requires a functional CHD in cells, we utilized a knockdown and replacement protocol in order to measure the function of kinetochores during the first mitosis where cells contain mutated proteins (Miller et al., 2008). HeLa 
cells were synchronized using a double thymidine block. Endogenous Hec1/Ndc80 protein was removed using transfected siRNA oligos and replaced by co-transfection of a rescue plasmid encoding a siRNA-resistant Hec1/Ndc80 gene (Figure 4-1A). Cells were fixed 8-12 hours after thymidine release and rescued cells were identified by an EGFP tag engineered onto the C-terminus of $\mathrm{Hec} 1 / \mathrm{Ndc} 80$. Immunoblots demonstrated efficient knockdown of the endogenous protein concomitant with the expression of GFP-tagged Hec1 (Supplemental Figure 4-2A). Nearly all wild type rescued metaphase cells (as judged by DAPI staining) also expressed EGFP, further demonstrating the efficiency of our system (Supplemental Figure 4-2B). Immunofluorescence with anti-Hec1/Ndc80 antibodies confirmed that $\mathrm{Hec} 1 / \mathrm{Ndc} 80$ intensity levels were similar to endogenous concentrations in our rescued cells (Supplemental Figure 4-2C). We also used immunofluorescence to confirm that kinetochores are properly assembled (Supplemental Figure 4-3). In Hec1/Ndc80 knockdown cells, Hec1/Ndc80, Spc25 and CENP-E are largely absent from kinetochores, and BubR1 is present but at reduced levels. The more centromere-proximal KNL-1 and ACA signals are present in knockdown cells. In $\mathrm{Hec} 1^{\mathrm{WT}}, \mathrm{Hec}^{\mathrm{K} 89 \mathrm{E} / \mathrm{K} 1115 \mathrm{E}}$ and Hec1 ${ }^{\mathrm{K} 89 \mathrm{E} / \mathrm{K} 166 \mathrm{E}}$ cells, all proteins localized to kinetochores. We conclude that kinetochores assemble properly after knockdown of the endogenous Hec1/Ndc80 and replacement with our CHD mutants. 
Figure 4-1: The CHD of Hec1/Ndc80 is essential for congression of chromosomes to the metaphase plate

(A) Scheme used to visualize the first mitotic division after endogenous Hec1/Ndc80 is replaced by exogenous Hec1/Ndc80 in synchronized HeLa cells. (B) Ribbon diagram depicting the N-terminal CHD regions of Hec1/Ndc80 (light blue, amino acids 80-285) and Nuf2 (yellow, amino acids 4-169). Lysine residues altered in mutants are shown in dark blue. The predicted exit point of a tubulin E-hook is indicated in red. Diagram was constructed based on the crystal structure of $\mathrm{Ndc} 80^{\text {bonsai }}$ (Cifferi, 2008) bound to a microtubule (Alushin et al., 2010). (C) Representative images of the predominant mitotic figures from siHec1 knockdown and rescued cells that have been stained for Hec1/Ndc80 (green), tubulin (red) and DNA (blue). WT and CHD mutant cells are identified by an expressed EGFP, and are co-stained for tubulin and DNA. For mock treated, Hec $1^{\text {WT }}$, $\mathrm{Hec} 1^{\mathrm{K} 89 \mathrm{E}}$ and $\mathrm{Hec} 1^{\mathrm{K} 115 \mathrm{E}}$ cells, representative metaphase images are shown. For siHec1, $\mathrm{Hec} 1^{\mathrm{K} 166 \mathrm{E}}, \mathrm{Hec} 1^{\mathrm{K} 89 \mathrm{E} / \mathrm{K} 115 \mathrm{E}}$ and Hec1 ${ }^{\mathrm{K} 89 \mathrm{E} / \mathrm{K} 166 \mathrm{E}}$, prometaphase cells are shown. (D) Mitotic cells were scored for chromosome alignment into a metaphase plate and the percentage of metaphase cells was plotted. Metaphase cells were further subdivided to indicate cells with all chromosomes aligned (black), cells with 1-2 unaligned chromsomes (gray) and cells with 3-5 unaligned chromosomes (white). N=3, 100 cells per experiment. Error bars $=\mathrm{SD}$ of total metaphase cell population. $(\mathrm{E})$ Cells were incubated in ice-cold media prior to fixation and immunostaining. Ten sister kinetochores from 5-10 cells $(\mathrm{N}=3)$ were scored for kinetochore-embedded microtubules and the mean percentage was plotted. Error bars $=\mathrm{SD}$. 
A

First thymidine block

$\downarrow 24 \mathrm{hr}$.

Release block, siRNA and DNA transfection

$\downarrow 12 \mathrm{hr}$.

Second block, siRNA transfection

$\downarrow 12 \mathrm{hr}$.

Release block

$\downarrow$ 8-12 hr.

Fix for immunofluorescence

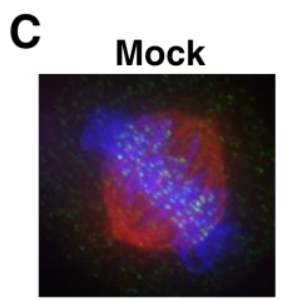

$\mathrm{Hec}^{\mathrm{K} 115 \mathrm{E}}$
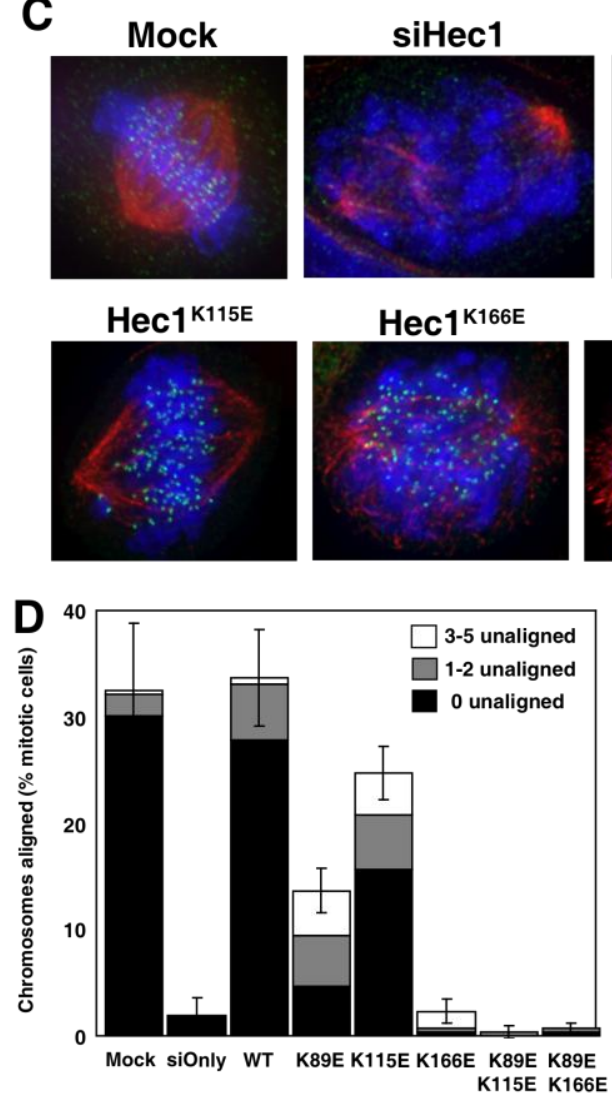

B

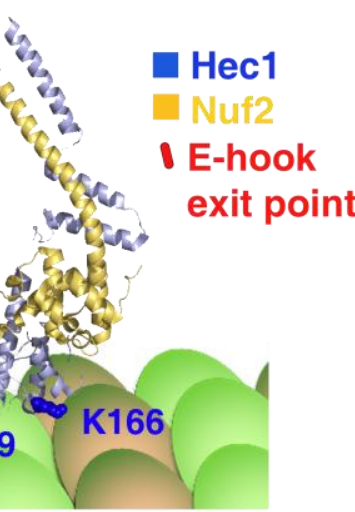

K115
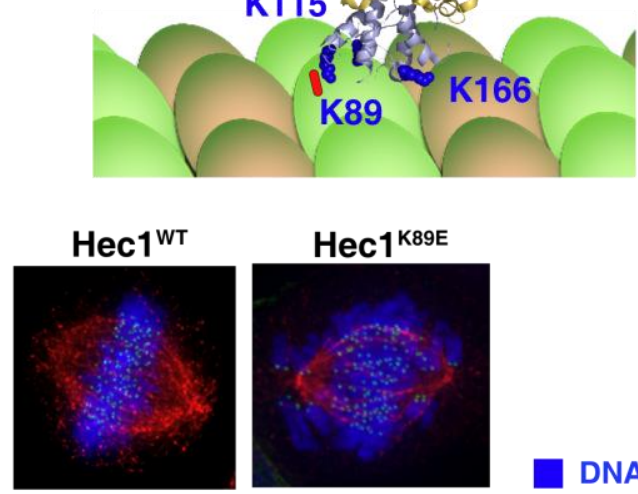

Hec1 $1^{\text {K89EK115E }}$

Hec1 $1^{\text {K89EK166E }}$
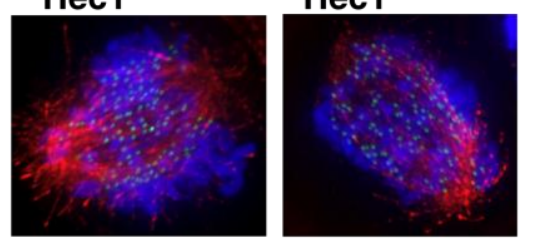

DNA

Hec1/GFP

Tubulin

E

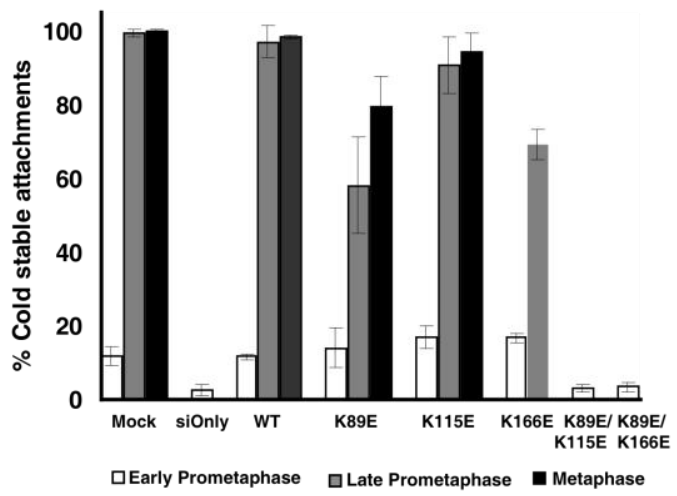

Figure 4-1 


\section{Kinetochores require the Hec1/Ndc80 CHD to bind microtubules}

We tested if kinetochores containing $\mathrm{Hec} 1 / \mathrm{Ndc} 80 \mathrm{CHD}$ mutants could properly align chromosomes in vivo. Cells were fixed and stained for tubulin and DNA. Mock transfected and Hec1/Ndc80 knockdown cells were also stained with anti-Hec1/Ndc80 antibodies, while Hec1/Ndc80 in rescued cells was identified with anti-GFP antibodies. Cells were scored for their ability to congress chromosomes to form a metaphase plate. Metaphase was defined as the majority of chromosomes in the center of the cell (more than five unaligned were scored as late prometaphase), although the pole-to-pole width of the plate varied greatly. In mock transfected and Hec $1^{\mathrm{WT}}$ cells, more than $30 \%$ of mitotic cells were able to achieve proper chromosome alignment at the time of fixation (Figure 41C and 4-1D). Only $2 \%$ of the mitotic cells were able to form a metaphase plate after Hec1/Ndc80 siRNA-treatment and rescue with an empty EGFP-expressing plasmid, consistent with published results (Guimaraes et al., 2008; Miller et al., 2008).

Replacement of endogenous $\mathrm{Hec} 1 / \mathrm{Ndc} 80$ with either double mutant resulted in an alignment defect identical to removing the entire protein. Similarly, only $2.3 \pm 1.2 \%$ of $\mathrm{Hec} 1^{\mathrm{K} 166 \mathrm{E}}$ cells contained aligned chromosomes, demonstrating that lysine 166 is a critical residue for $\mathrm{Hec} 1 / \mathrm{Ndc} 80$ function in vivo.

We identified intermediate phenotypes in the K115E and K89E mutants, which bind microtubules near the predicted exit point of tubulin E-hooks (Alushin et al., 2010). $\mathrm{Hec} 1^{\mathrm{K} 115 \mathrm{E}}$ mutant cells were able to largely restore metaphase alignment, with $24.7 \pm$ $2.5 \%$ of mitotic cells scored as aligned (Figure 4-1C and 4-1D). Cells expressing the $\mathrm{Hec} 1^{\mathrm{K} 89 \mathrm{E}}$ mutant had a more severe phenotype, with $13.7 \pm 2.1 \%$ of cells scored as aligned. In addition to lower percentages of metaphase cells compared to controls we saw 
additional spindle defects in these cells. First, although $5.3 \%$ of $\mathrm{Hec}^{\mathrm{WT}}$ expressing cells are found in anaphase we could not identify any CHD mutant cells in anaphase. This suggests that cells containing CHD mutants either cannot bind chromosomes well enough to fulfill the spindle checkpoint or cannot segregate chromosomes in anaphase. We found that an informative way to measure more subtle alignment defects was to identify cells with metaphase plates and quantify the number of chromosomes that remain at or near the poles (Figure 4-1D). In the mock-treated conditions or cells rescued with $\mathrm{Hec}^{\mathrm{WT}}$, more than $82 \%$ of metaphase cells had all their chromosomes aligned at the center. In contrast, $67 \%$ of $\mathrm{Hec} 1^{\mathrm{K} 89 \mathrm{E}}$ or $35 \%$ of $\mathrm{Hec} 1^{\mathrm{K} 115 \mathrm{E}}$ metaphase cells had unaligned chromosomes. We conclude that the K115E and K89E mutants have similar intermediate phenotypes and note that the double mutant is more severe. This suggests that these two residues share a common function.

After kinetochores generate bipolar attachments, they use the bound microtubules to generate force and pull sisters apart (Maddox et al., 2003). The distance between sisters (inter-kinetochore distance) can be used as a readout of the forces generated. In early prometaphase cells, which presumably have few kinetochore-microtubule attachments, all of the cell populations were similar to nocodazole-treated cells $(0.68 \pm$ $0.01 \mu \mathrm{m})$ (Supplemental Figure 4-4A). CHD double point mutant cells remained in early prometaphase and could not separate sister chromatids. Hec $1^{\mathrm{K} 89 \mathrm{E}}(1.11 \pm 0.04 \mu \mathrm{m})$ and $\mathrm{Hec}^{\mathrm{K} 115 \mathrm{E}}(1.21 \pm 0.02 \mu \mathrm{m})$ cells in metaphase generated intermediate inter-kinetochore distances that were reduced compared to mock or Hec $1^{\mathrm{WT}}$ metaphase cells $(1.40 \pm 0.08$ $\mu \mathrm{m}$ and $1.36 \pm 0.04 \mu \mathrm{m}$, respectively). A percentage of $\mathrm{Hec} 1^{\mathrm{K} 166 \mathrm{E}}$ cells were also able to progress to late prometaphase, and these cells had an average inter-kinetochore distance 
of $1.01 \pm 0.04 \mu \mathrm{M}$. Therefore, kinetochores from CHD mutant cells cannot effectively utilize microtubules to generate maximal pulling forces on sister kinetochores. Like the alignment defects, there is a gradation in these phenotypes, with $\mathrm{Hec} 1^{\mathrm{K} 166 \mathrm{E}}$ cells able to generate the least amount of force and $\mathrm{Hec} 1^{\mathrm{K} 115 \mathrm{E}}$ cells showing the smallest diminishment of force production.

Kinetochore-microtubule attachments are resistant to cold temperature, a treatment that depolymerizes most microtubules in a mitotic cell (Brinkley and Cartwright, 1975). Control and CHD mutant cells were incubated in ice-cold media prior to fixation and stained for tubulin, $\mathrm{Hec} 1 / \mathrm{Ndc} 80$ (or GFP), and ACA (see images in Supplemental Figure 4-5). The percentage of kinetochores with embedded cold stable microtubule bundles (K-fibers) was scored. The double mutants acted almost identically to a Hec1/Ndc80 knockdown cell, and the single mutants generated a gradation of phenotypes. Hec $1^{\mathrm{K} 115 \mathrm{E}}$ and $\mathrm{Hec} 1^{\mathrm{K} 89 \mathrm{E}}$ mutant cells generated cold stable attachments at $94.0 \pm 5.3 \%$ and $79.3 \pm 8.1 \%$ of kinetochores during metaphase, demonstrating that most kinetochores containing these mutants are able to bind microtubules. Interestingly $69.3 \pm$ $4.2 \%$ of $\mathrm{Hec}^{\mathrm{K} 166 \mathrm{E}}$ cells had cold stable attachments in metaphase. This demonstrates that these kinetochores were able to bind microtubules at reduced levels but can't use the attachments to align chromosomes.

In conclusion all three CHD residues that we tested play important roles in generating tension and aligning chromosomes, although kinetochores containing the single mutants appear able to attach microtubules with some efficiency. All three residues make independent contributions to kinetochore function, as double mutants are more severe and cannot stabilize microtubules against cold-induced depolymerization. 
CHD mutant phenotypes cannot be rescued by inhibiting Aurora B phosphorylation of the Hec1/Ndc80 tail

Phosphorylation of the Hec1/Ndc80 N-terminal tail by Aurora B lowers its affinity for microtubules in vitro and is believed to be a critical step for kinetochores to release improper attachments in vivo (Cheeseman et al., 2006; Ciferri et al., 2008; Guimaraes et al., 2008; Welburn et al., 2010; Alushin et al., 2010). Therefore, we wondered if the reason our CHD mutants were not aligning chromosomes is because they trigger phosphorylation of the $\mathrm{Hec} 1 / \mathrm{Ndc} 80$ tail. This would presumably weaken kinetochore interactions with microtubules and indirectly cause the phenotypes we observe. To test this, we generated a trio of Hec1/Ndc80 CHD mutants that also has nine consensus Aurora B phosphorylation sites on the tail mutated to alanine ( $\mathrm{Hec} 1^{9 \mathrm{~A} / \mathrm{K} 89 \mathrm{E}}$, Hec $1^{9 \mathrm{~A} / \mathrm{K} 166 \mathrm{E}}, \mathrm{Hec} 1^{\text {9A/K89EK166E }}$ ) (sites highlighted in Figure 4-2A). As a control for the role of phosphorylation, we created a phospho-null mutant $\left(\mathrm{Hec} 1^{9 \mathrm{~A}}\right)$ that contains the nine mutated phosphorylation sites but lacks mutations in the CHD.

HeLa cells can generate productive microtubule attachments, generate interkinetochore tension and align chromosomes after knockdown and replacement with $\mathrm{Hec}^{9 \mathrm{~A}}$ mutants (Figure 4-2B and 4-2C). We did notice a slight increase in unaligned chromosomes in our $\mathrm{Hec}^{9 \mathrm{~A}}$ cells as well as an increase in inter-kinetochore distance (Supplemental Figure 4-4B), in agreement with published data (Deluca et al., 2006). However the Hec1 ${ }^{9 \mathrm{~A} / \mathrm{K} 89 \mathrm{E} / \mathrm{K} 166 \mathrm{E}}$ mutant cells produced a phenotype that recapitulated the $\mathrm{Hec} 1^{\mathrm{K} 89 \mathrm{E} / \mathrm{K} 166 \mathrm{E}}$ mutant. Only $0.3 \pm 0.6 \%$ of these mutant cells had metaphase-aligned chromosomes (Figure 4-2B and 4-2C). Furthermore, they could not generate stretch between sister kinetochores (Supplemental Figure 4-4B) and could not stabilize microtubules against cold-induced depolymerization (Figure 4-2D). We conclude that 
kinetochores cannot generate K-fibers using tail interactions alone, but they require a functional CHD.

In contrast, the phenotypes generated in our Hec $1^{\mathrm{K} 89 \mathrm{E}}$ and $\mathrm{Hec} 1^{\mathrm{K} 166 \mathrm{E}}$ cells were improved by removing Aurora sites from the Hec1/Ndc80 tail. Approximately twice as many Hec $1^{9 \mathrm{~A} / \mathrm{K} 89 \mathrm{E}}$ mitotic cells formed a metaphase plate than $\mathrm{Hec} 1^{\mathrm{K} 89 \mathrm{E}}$ cells (compare Figure 4-1D with Figure 4-2C). In addition Hec1 ${ }^{9 \mathrm{~A} / \mathrm{K} 89 \mathrm{E}}$ metaphase cells had a similar number of stabilized microtubules compared to $\mathrm{Hec} 1^{\mathrm{WT}}$ and $\mathrm{Hec} 1^{9 \mathrm{~A}}$ controls (Figure 42D), although there was less rescue of their ability to generate forces to separate sister kinetochores (Supplemental Figure 4-4B). Creating an unphosphorylated tail also partially rescued the $\mathrm{Hec} 1^{\mathrm{K} 166 \mathrm{E}}$ mutation (Figure 4-2, Supplementary Figure 4-4B); but here again the 9A/K166E mutation was more severe than the 9A/K89E. We conclude that Aurora B phosphorylation of the $\mathrm{Hec} 1 / \mathrm{Ndc} 80$ tail does contribute to the intermediate phenotypes we see in our single mutants, but the main reason for the phenotype is impaired interaction between the Hec1/Ndc80 CHD and a microtubule. 
Figure 4-2: Hec1/Ndc80 CHD mutant phenotypes cannot be rescued by preventing Aurora phosphorylation of the Hec1/Ndc80 tail

(A) Sequence of the 80 amino acid Hec1/Ndc80 tail region. Nine wild type resdiues were mutated to alanine $\left(\mathrm{Hec}^{9 \mathrm{~A}}\right)$ and are indicated in red. (B) Representative images of predominant mitotic figures from siHec1 knockdown and rescued cells that have been stained for Hec1/Ndc80, tubulin and DNA. For Hec1 $1^{\mathrm{WT}}, \mathrm{Hec} 1^{9 \mathrm{~A}}, \mathrm{Hec} 1^{9 \mathrm{~A} / \mathrm{K} 89 \mathrm{E}}$ and Hec1 $1^{9 \mathrm{~A} / \mathrm{K} 166 \mathrm{E}}$, metaphase images are shown. For Hec1 $1^{9 \mathrm{~A} / \mathrm{K} 89 \mathrm{E} / \mathrm{K} 166 \mathrm{E}}$, only prometaphase cells are observed. (C) Rescued mitotic cells were stained for tubulin and DNA, identified by an EGFP kinetochore signal and scored for chromosome alignment. The percentage of metaphase aligned cells was plotted in comparison to mock-transfected cells. $\mathrm{N}=3 ; \geq 100$ cells per experiment. Metaphase cells were further subdivided to indicate cells with all chromosomes aligned (black), cells with 1-2 unaligned chromsomes (gray) and cells with 3-5 unaligned chromosomes (white). Error bars = SD of total metaphase cell population. (D) Cells were incubated in ice-cold media prior to fixation and immunostaining with tubulin and ACA antibodies. Ten kinetochores in 5-10 cells $(\mathrm{N}=3)$ were scored for percentage of kinetochores containing embedded microtubules and the mean percentage was plotted. Error bars $=\mathrm{SD}$. 
A WT 1-MKRSSVSSGG AGRLSMQELR SQDVNKQGLY TPQTKEKPTF- 40 41- GKLSINKPTS ERKVSLFGKR TSGHGSRNSQ LGIFSSSEKI-80

B

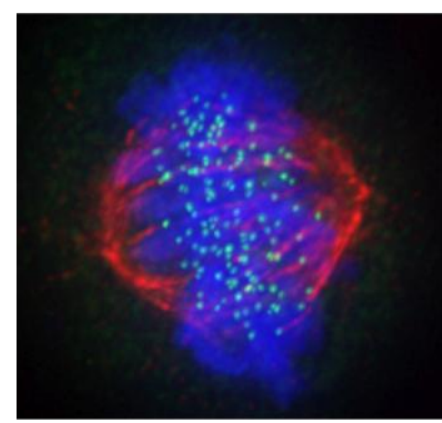

$\mathrm{Hec}^{\mathrm{WT}}$

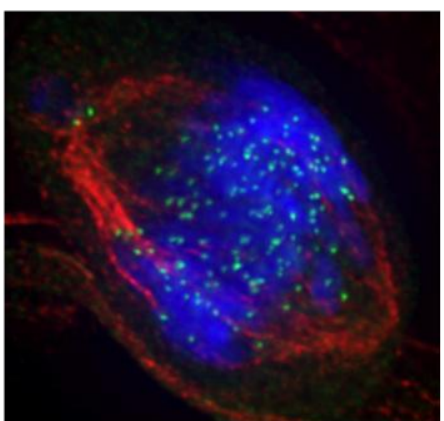

Hec1 $1^{9 A / K 89 E}$

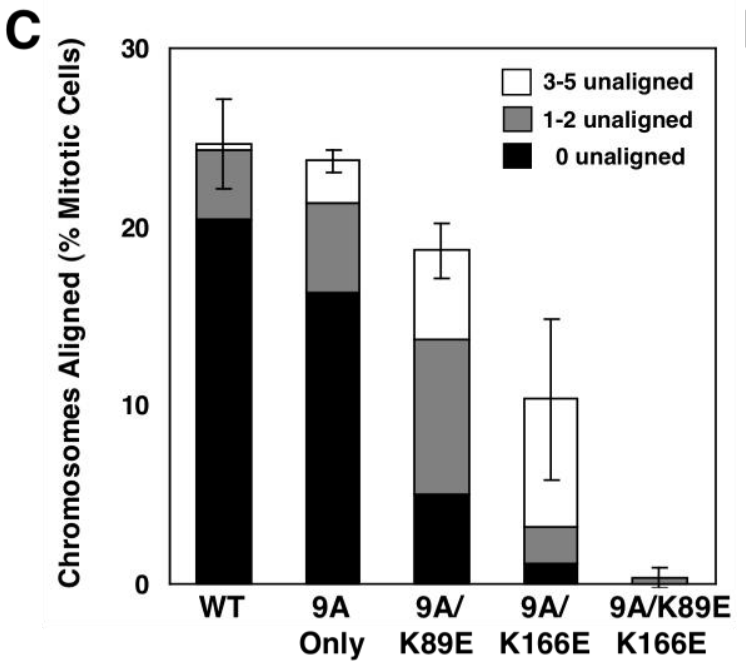

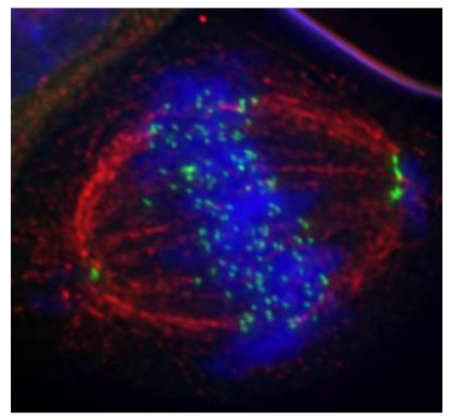

$\operatorname{Hec}^{9 \mathrm{~A}}$

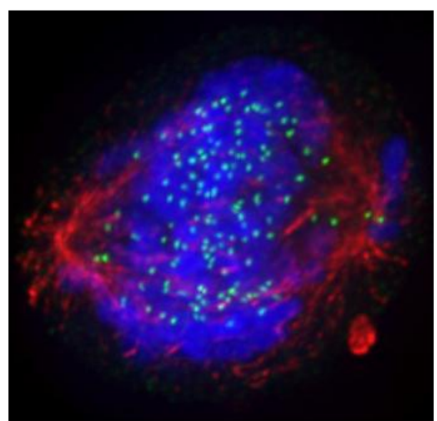

Hec19A/K166E

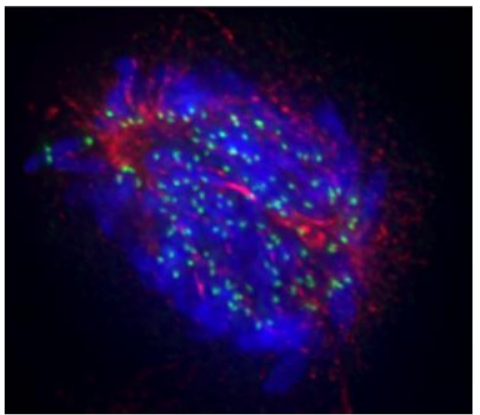

Hec19A/K89E/K166E
DNA

Hec1-GFP

Tubulin

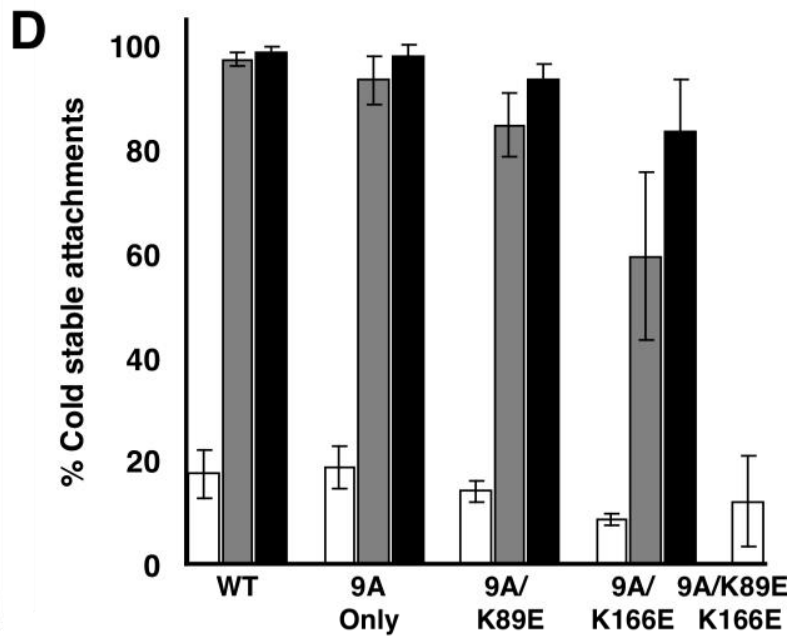

口Early Prometaphase $\square$ Late Prometaphase Metaphase

Figure 4-2 


\section{Both the Hec1/Ndc80 CHD and the N-terminal tail utilize charge to interact with microtubules}

Phenotypes resulting from lysine mutation could be due to loss of positive charge or loss post-translational modifications on the lysine. To distinguish between these models we tested the contribution of charge. Mutants were generated that replaced a pair of lysines in the CHD with either glutamic acid ( $\left.\mathrm{Hec} 1^{\mathrm{K} 89 \mathrm{E} / \mathrm{K} 166 \mathrm{E}}\right)$, alanine $\left(\mathrm{Hec} 1^{\mathrm{K} 89 \mathrm{~A} / \mathrm{K} 166 \mathrm{~A}}\right)$ or arginine $\left(\mathrm{Hec} 1^{\mathrm{K} 89 \mathrm{R} / \mathrm{K} 166 \mathrm{R}}\right)$. Whereas Hec1 ${ }^{\mathrm{K} 89 \mathrm{R} / \mathrm{K} 166 \mathrm{R}}$ mutant cells (which retained the charge state) were able to restore metaphase alignment neither Hec $1^{\mathrm{K} 89 \mathrm{~A} / \mathrm{K} 166 \mathrm{~A}}$ nor $\mathrm{Hec} 1^{\mathrm{K} 89 \mathrm{E} / \mathrm{K} 166 \mathrm{E}}$ expressing cells could align chromosomes to form a metaphase plate (Figures 4-3A and 4-3B). In contrast, $\mathrm{Hec} 1^{\mathrm{K} 89 \mathrm{R} / \mathrm{K} 166 \mathrm{R}}$ cells could progress into anaphase (data not shown), generate sufficient inter-kinetochore tension (Supplemental Figure 44C), and stabilize microtubules against cold-induced depolymerization (Figure 4-3C). We conclude that positive charge in the side chains of these amino acids is critical for kinetochore function.

While Hec $1^{\mathrm{K} 89 \mathrm{~A} / \mathrm{K} 166 \mathrm{~A}}$ mutant cells could not align chromosomes efficiently enough to form a metaphase plate, some chromosomes formed cold stable attachments and generated inter-kinetochore tension at reduced levels. No such attachments were seen in the $\mathrm{Hec} 1^{\mathrm{K} 89 \mathrm{E} / \mathrm{K} 166 \mathrm{E}}$ expressing cells (Figure 4-3C and Supplemental Figure 4-4C). These data demonstrate a gradation of phenotypes as the positively charged lysines in the CHD are replaced with positive, neutral and negatively charged residues. 
Figure 4-3: The interaction between the Hec1/Ndc80 CHD and a microtubule requires charge

(A) Representative images of the predominant phenotype of Hec1/Ndc80 CHD mutant cells. For Hec $1^{\mathrm{K} 89 \mathrm{E} / \mathrm{K} 166 \mathrm{E}}$ mutant cells, only early prometaphase figures were found. Both early prometaphase and late prometaphase (shown) figures were found in $\mathrm{Hec} 1^{\mathrm{K} 89 \mathrm{~A} / \mathrm{K} 166 \mathrm{~A}}$ mutant cells. For Hec1 $1^{\mathrm{K} 89 \mathrm{R} / \mathrm{K} 166 \mathrm{R}}$ mutant cells, both prometaphase and metaphase (shown) figures were observed. (B) Mitotic cells were scored for chromosome alignment and the percentage of metaphase aligned cells was plotted. $\mathrm{N}=3 ; \geq 100$ cells counted each experiment. (C) Cells were incubated in ice-cold media prior to fixation and immunostaining with tubulin and ACA antibodies. Mock treated and siHec1 knockdown cells also were stained for Hec1/Ndc80 (9G3). Ten kinetochores in 5-10 cells ( $\mathrm{N}=3$ ) were scored for the percentage of kinetochores containing embedded microtubules and the mean percentage was plotted. Error bars $=\mathrm{SD}$. 


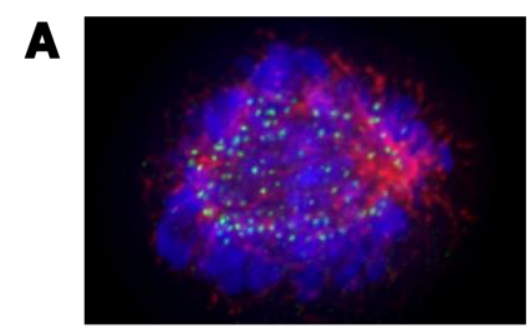

Hec1 ${ }^{\text {K89EK166E }}$

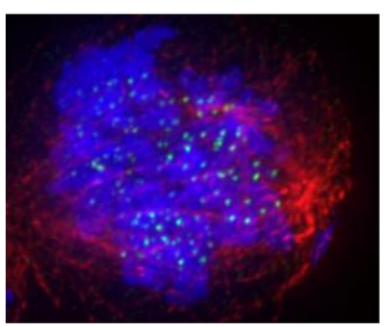

Hec1 ${ }^{\mathrm{K} 89 \mathrm{AK} 166 \mathrm{~A}}$

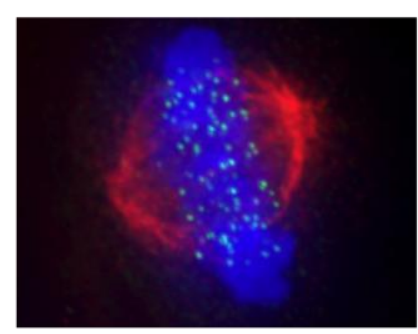

Hec1 K89RK166R

DNA Hec1/GFP Tubulin
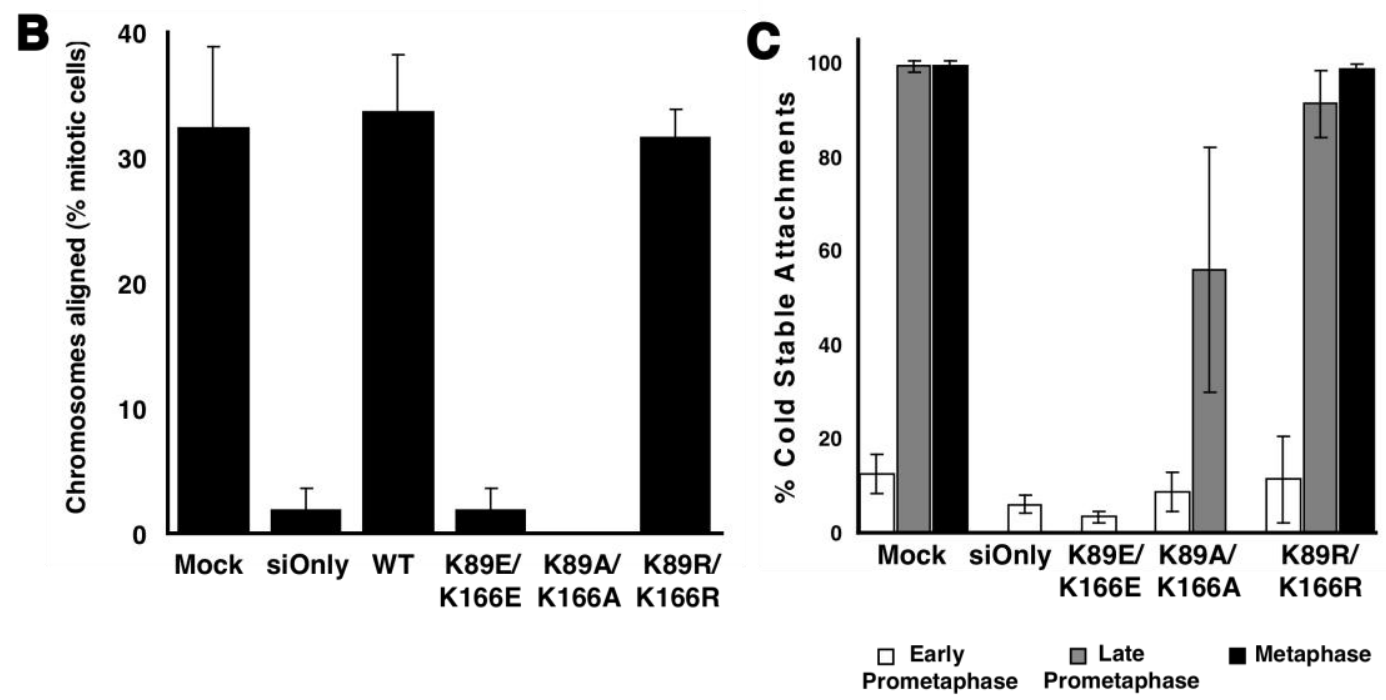

Figure 4-3 
A Hec1/Ndc80 mutant lacking the $\mathrm{N}$-terminal tail region $\left(\mathrm{Hec}^{\Delta \mathrm{N}}\right)$ is unable align chromosomes in vivo (Guimaraes et al., 2008; Miller et al., 2008). This 80 amino acid region contains 15 positively charged and five negatively charged residues spread across its length. To test the contribution of charge to Hec1/Ndc80 tail binding to microtubules, we tested a charge-neutral protein $\left(\mathrm{Hec}^{\mathrm{NEU}}\right)$ where ten positively charged residues were mutated to alanine (Figure 4-4A). This reduces the net charge of the tail from +10 to 0 . Recombinant Ndc80 $0^{\text {bonsai }}$ containing a charge-neutral tail lost much of its ability to bind microtubules in vitro (Figure 4-4B). While charge-neutral $\mathrm{Ndc}^{\text {bonsai }}$ pelleted better than wild type at lower microtubule concentrations, the binding curve reached a plateau and never exceeded $34.9 \%$ bound - demonstrating that charge on the N-terminal tail of Hec1/Ndc80 stabilizes the interaction between the Ndc80 complex and microtubules. The increased binding of the complex at lower tubulin concentrations also suggests that cooperative binding is lost in this mutant.

The Hec1 ${ }^{\mathrm{NEU}}$ mutant was tested in vivo using our knockdown and replacement protocol. Hec $1^{\mathrm{NEU}}$ mutant cells were unable to align chromosomes and form a metaphase plate (Figure 4-4C and 4-4D). Hec1 ${ }^{\mathrm{NEU}}$ mutant cells remained in early prometaphase with few $(14.9 \pm 1.2 \%)$ cold-stable microtubules (Figure 4-4E) and kinetochores that were unable to generate forces to stretch sisters apart (average inter-kinetochore distance of $0.79 \pm 0.07 \mu \mathrm{m})($ Figure $4-4 \mathrm{~F})$. Kinetochore assembly was unperturbed in $\mathrm{Hec} 1^{\mathrm{NEU}}$ mutant cells (Supplemental Figure 4-6). Therefore, the charge on the N-terminal unstructured region of $\mathrm{Hec} 1 / \mathrm{Ndc} 80$ is critical for kinetohores to bind microtubules and align chromosomes. 
Figure 4-4: Positive charge in unstructured Hec1/Ndc80 tail is required for microtubule binding and chromosome congression

(A) Amino acid sequence of charge neutral tail Hec1 mutant with mutated residues indicated in red. (B) WT and charge neutral Hec1 tail Ndc80 ${ }^{\text {bonsai }}$ were sedimented with the indicated concentrations of microtubules and pellet samples $(\mathrm{N}=3)$ were subjected to western blotting with anti-hSpc25 antibody. Signal intensities were quantified and the mean percentage of $\mathrm{Ndc} 80^{\text {bonsai }}$ bound at each microtubule concentration was plotted on a $\log$ and linear scale (inset). Error bars $=$ SD. (C) Representative image of a knocked down cell that has been rescued with $\mathrm{Hec} 1^{\mathrm{NEU}}$. The cell is stained for Hec1/Ndc80 (green), tubulin (red) and DNA (blue). In this condition only early prometaphase figures are found. (D) Mitotic cells were scored for kinetochore alignment $(n \geq 100)$ and the percentage of mitotic cells with aligned chromosomes was plotted. (E) Ten kinetochores from five or more cells $(n>50)$ were scored for kinetochore associated microtubules $(\mathrm{N}=2)$, and the mean percentage in early prometaphase, late prometaphase and metaphase was plotted. (F) Ten sister kinetochores in at least five cells $(n>50)$ were identified by ACA staining between $\mathrm{Hec} 1 / \mathrm{Ndc} 80$ signals and the distance between those sister kinetochores was measured $(\mathrm{N}=3)$. The mean distance is plotted for early prometaphase and metaphase cells. Error bars $=\mathrm{SD}$. 


\section{A WT 1-MKRSSVSSGG AGRLSMQELR SQDVNKQGLY TPQTKEKPTF- 40 41-GKLSINKPTS ERKVSLFGKR TSGHGSRNSQ LGIFSSSEKI-80}
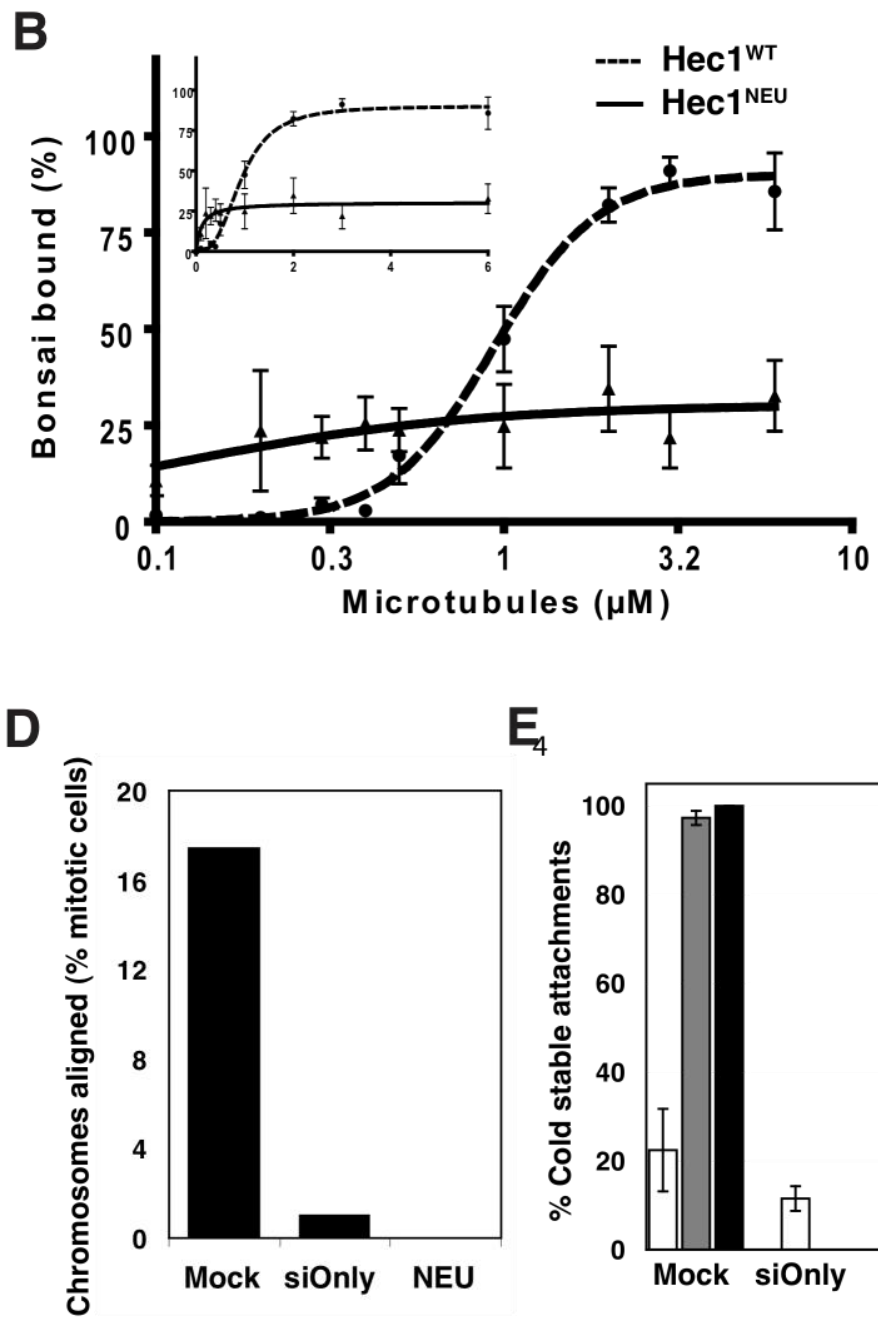

$\mathbf{E}_{4}$

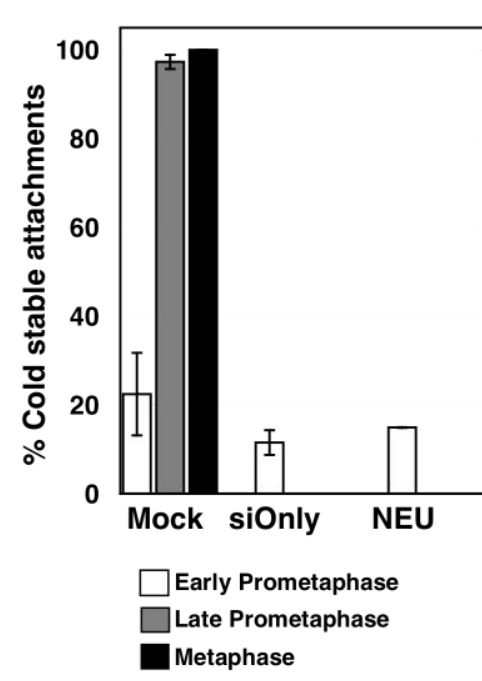

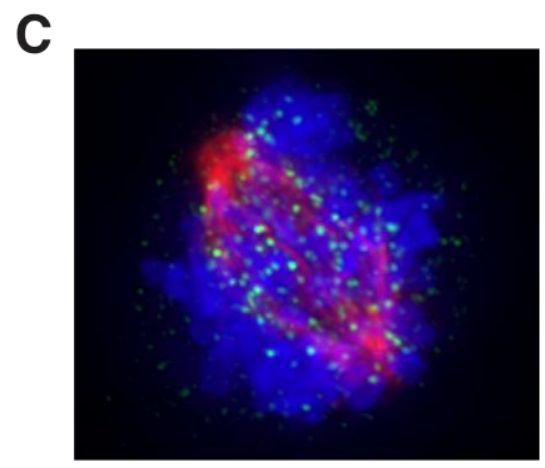

$\mathrm{Hec}^{\text {NEU }}$

DNA Hec1/GFP

Tubulin

$\mathbf{F}$

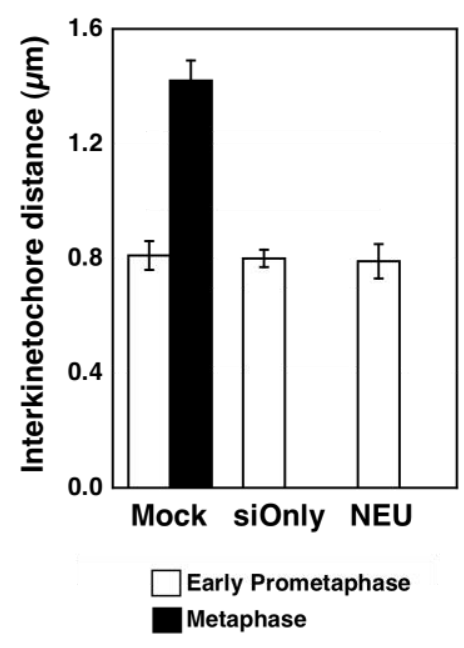

Figure 4-4 


\section{Discussion}

Our data provide significant insight into the mechanism by which vertebrate kinetochores couple conformational changes generated by microtubule depolymerization into the energy to move chromosomes. Like the unstructured N-terminal tail, the CHD of $\mathrm{Hec} 1 / \mathrm{Ndc} 80$ is essential for binding microtubules and congressing chromosomes in vivo. Hec1/Ndc80 knockdown cells rescued with CHD point mutants fail to align chromosomes, generate tension across sister kinetochores or produce stable microtubule attachments. Interactions with microtubules are mediated by charge, since lysines can be functionally replaced by arginines, but not by alanines or glutatmic acid. We also extend our studies of the Hec1/Ndc80 tail by demonstrating that the large amounts of positive charge residing in this unstructured region drive proper kinetochore function. A formal possibility was that the CHD phenotypes we observed were indirectly caused by activation of the microtubule release system, which includes Aurora B phosphorylation of the tail of Hec1/Ndc80 and additional KMN components (Welburn et al., 2010). We tested this by combining mutations in both the CHD and regulatory sites in the tail, and although there may be some triggering of the release system, our data suggest a direct role for the CHD binding to the microtubule in chromosome congression. Taken together, we conclude that electrostatic contacts dictate the interaction between Hecl and a microtubule in vivo. This interaction is dependent on the two positively charged regions of the Hec1/Ndc80 CHD and is facilitated by additional electrostatic interactions on the tail. 


\section{The Hec1/Ndc80 CHD has two distinct microtubule-binding motifs in vitro and both are required in vivo}

We have demonstrated that three lysine residues implicated in microtubule binding in vitro (Ciferri et al., 2008) are critical for kinetochore function in vivo. The recent publication of a high-resolution cryo-EM structure of the $\mathrm{Ndc} 80^{\text {bonsai }}$ on microtubules provided significant insight into Ndc80 function (Alushin et al., 2010) and allowed us to greatly refine the interpretation of our work. The residues that generated phenotypes are located in two distinct regions of the Hec1/Ndc80 CHD. In the crystal structure of $\mathrm{Ndc} 80^{\text {bonsai }}$, lysine 166 resides in the "toe" portion of the CHD that positions the $\mathrm{Ndc80}$ complex at the interface between tubulin dimers and is proposed to work as a microtubule conformation sensor (Alushin et al., 2010). It is also located adjacent to the serine 165 residue, which is proposed to be a site of Nek2A regulation (Du et al., 2008). In contrast, lysines 89 and 115 reside away from the toe region of $\mathrm{Hec} 1 / \mathrm{Ndc} 80$ but close to the predicted exit point for negatively charged E-hooks (Alushin et al., 2010). As tubulin tails were too unstructured to be captured in the structural data, we cannot definitively say that lysines directly contact E-hooks. However, we note that the closest negatively charged group emanating from the folded portion of a tubulin subunit is more than $10 \AA$ from either lysine 89 or 115 . This strongly suggests that these CHD residues are in contact with E-hooks, and not the structured tubulin subunit. Thus, our in vivo data strongly support the findings and many of the interpretations of the recent cryo-EM structure.

When we mutate each of these three residues alone we find that mutation of lysine 166 generates the most severe phenotype, demonstrating that the toe region of $\mathrm{Hec} 1 / \mathrm{Ndc} 80$ is critical for proper kinetochore function in vivo. Mutation of lysine 89 or 
lysine 115 alone generates more intermediate phenotypes, while mutating these two residues in tandem is far more severe. These data could be interpreted in two ways. One interpretation is that lysines 89 and 115 are both implicated in binding E-hooks, and in the absence of one contact point the other is able to maintain a certain level of contact with this region of a microtubule. However, when both contact points are lost the $\mathrm{Hec} 1 / \mathrm{Ndc} 80 \mathrm{CHD}$ is now unable to interact with E-hooks and all microtubule binding is lost. Alternatively, a genetic argument could be made that each of these residues has an independent function. We note that E-hooks have 10-12 acidic residues (Hiser et al., 2006), and both lysine 89 and 115 lie close to the predicted E-hook emergence point. As there are no other nearby amino acids from the structured tubulin subunit to generate saltbridges, we propose a model where both lysine 89 and 115 simultaneously interact with different glutamic acids on a single E-hook (Figure 4-5).

\section{The role of the unstructured Hec1/Ndc80 tail}

In vertebrates the $\mathrm{Hec} 1 / \mathrm{Ndc} 80$ tail is required for kinetochore function in vivo (Guimaraes et al., 2008; Miller et al., 2008). However, the in vivo alignment phenotype we see in our paired CHD point mutants (where the tail is intact) is just as dramatic as removing the entire tail or eliminating its positive charge. Thus both the CHD and the tail are absolutely essential. However, the role for the tail remains somewhat unclear. The Hec1 tail binds microtubules better than the CHD in vitro, and it greatly increases the affinity of the CHD for microtubules. Our chargeless tail mutant reduces binding to microtubules in vitro and in vivo, strongly suggesting that additional microtubule binding by the tail facilitates binding. However, there is an alternative model suggested by the recent cryo-EM data - that the tail controls cooperative binding. This model is also 
supported by our data and we will discuss this below. Finally, the tail is a point of regulation by the Aurora B kinase - suggesting an additional role for this 80 amino acid region.

The Ndc80 complex binds microtubules in patches in vitro (Ciferri et al., 2008; Alushin et al., 2010), suggesting that there is a preference for an Ndc80 complex to bind to a tubulin subunit adjacent to another bound $\mathrm{Ndc} 80$ complex. The cryo-EM data show that $\mathrm{Ndc80}$ complexes tightly pack along a protofilament, and there is a significant binding interface between adjacent $\mathrm{Ndc} 80$ complexes. These data suggest a treadmill model for Ndc80 function, where binding of multiple Ndc80 subunits along a tubulin protofilament generates a tighter binding surface. As an Ndc80 complex is released from a tubulin subunit following depolymerization-induced protofilament curvature, rebinding of that released subunit behind the last bound $\mathrm{Ndc} 80$ could allow a treadmilling mechanism. This would provide the kinetochore with a tight binding interface that can also move with a depolymerizing microtubule. While most of the density in the cryoEM structure could be accounted for by the current crystal structure of a monomeric Ndc $80^{\text {bonsai }}$ complex which lacks the unstructured tail, there was extra density between adjacent Ndc80 subunits when the wild type complex was used that could represent the tail region of $\mathrm{Hec} 1 / \mathrm{Ndc} 80$. This suggests a role for the tail in driving tight packing of adjacent Ndc80 proteins along a protofilament, in support of the model discussed above (Figure 4-5A).

However, we also postulate an alternative role for the vertebrate $\mathrm{Hec} 1 / \mathrm{Ndc} 80$ tail based on our previous in vitro finding that the tail binds microtubules on it own and even shows significant cooperative binding in the absence of a Hec1/Ndc80 CHD (Miller et 
al., 2008). We propose that the tail may act as a tether to provide additional binding affinity following transient loss of CHD binding during periods of microtubule depolymerization. This would allow an Ndc80 complex to move from one tubulin subunit to another (Figure 4-5B). Because our Hec $1^{\mathrm{NEU}}$ both loses overall binding and also cooperative binding (which is presumably reflective of the tight packing of subunits), our data currently support either model. We note that the two models are not mutually exclusive, and we currently favor a model where both are correct. The Hec1/Ndc80 tail is 80 amino acids long, and assuming the tight packing model is correct we would estimate that less than 30 amino acids could be fit into the extra density identified by the cryo-EM structure. This would allow most of the tail to still act as a tether where it could both enhance microtubule binding affinity and facilitate the poleward transfer of an Ndc80 complex from a curvature-enacted release point (Figure 4-5B). It remains unclear how an exclusive role for the tail in packing Hec1/Ndc80 complexes would allow for the observed oscillations of chromosomes at the metaphase plate, where kinetochores must reposition themselves on polymerizing microtubules. Additionally, we speculate that intermediate amounts of Aurora B phosphorylation of the Hec1/Ndc80 tail could trigger a switch between a packing function and a tethering function. It is likely that an intermediate phosphorylation state would exist in prometaphase before kinetochore forces pull sister kinetochores apart. Finally, we note that despite implications for a role in binding to microtubules in vitro, the role of Nuf2 remains largely unanswered. Clearly, these are all important areas for future research. 
Figure 4-5: Model for vertebrate Hec1/Ndc80's interaction with a microtubule

(A) The unstructured tail of Hec1/Ndc80 (dark blue line) binds tubulin tails (red lines) and also acts to pack adjacent Ndc80 complexes on the surface of a microtubule. Lysine 166 of the $\mathrm{Hec} 1 / \mathrm{Ndc} 80 \mathrm{CHD}$ is a critical residue in the "toe" region of the protein, while tubulin E-hooks also interact with lysines 89 and 115 of the Hec1/Ndc80 CHD (Alushin et al., 2010). (B) Alternatively, the tail may function primarily to tether the Ndc80 complex to microtubules during periods when CHD binding is lost. The models depicted in (A) and (B) need not be mutually exclusive, and could be regulated by Aurora phosphorylation of the $\mathrm{Hec} 1 / \mathrm{Ndc} 80$ tail. For simplicity, only tubulin tails involved in binding are depicted. 

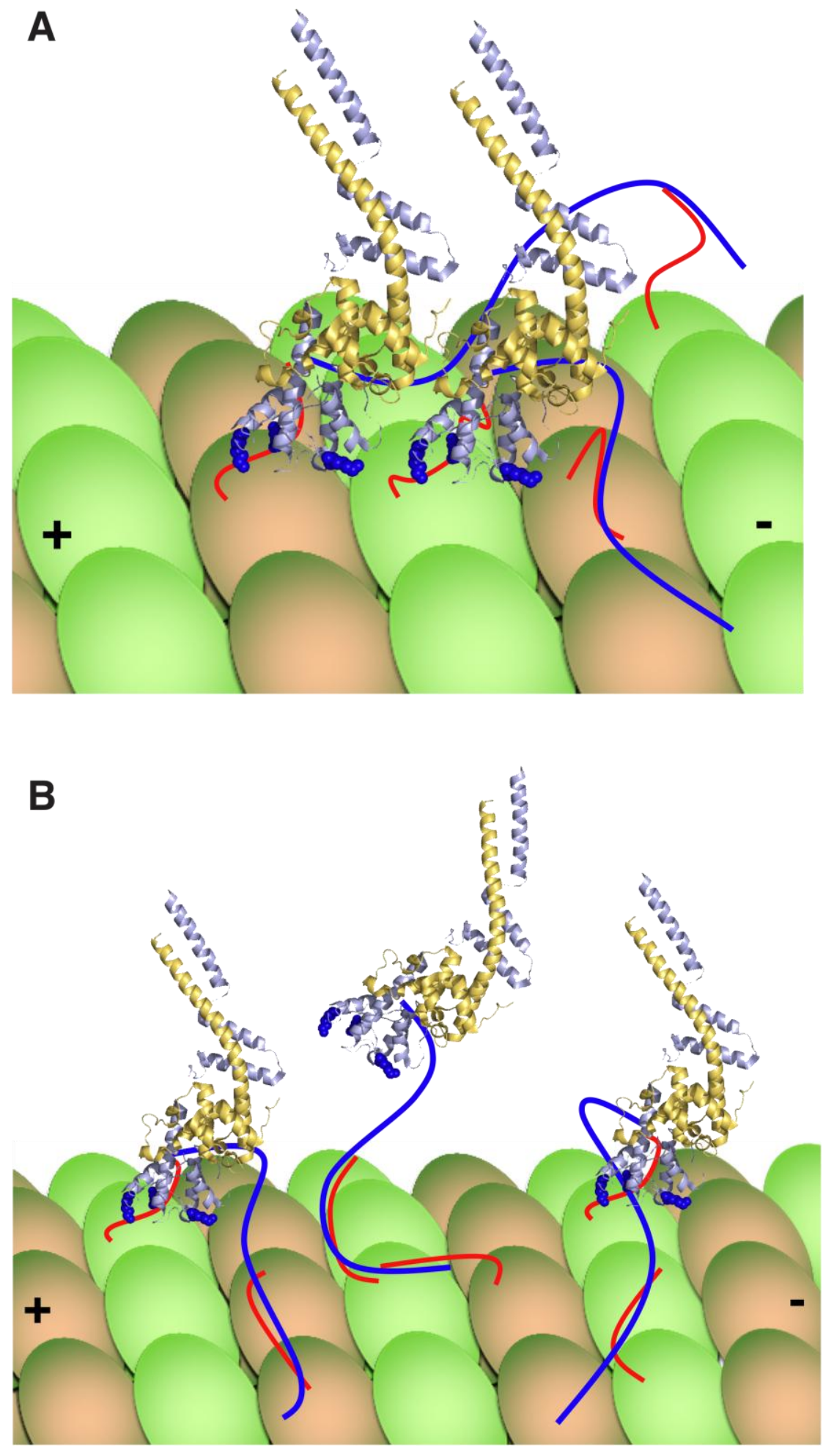

Figure 4-5 


\section{Acknowledgements}

We thank Dan Burke and Dan Foltz for critical reading of the manuscript. We also thank members of the Stukenberg lab, especially Pawel Janczyk, for helpful comments and discussions, and we thank members of the Foltz lab for their ongoing generosity. Finally, we are grateful to Andrea Musacchio (Ndc80 ${ }^{\text {bonsai }}$ constructs), Gary Gorbsky (Mad2 antibody), Arshad Desai (KNL-1 antibody), and Tim Yen (CENP-E antibody) for providing gift reagents. J.G.T. was supported by the Cancer Training Grant from the University of Virginia; S.A.M. was supported by the Cell and Molecular Biology Training Grant from the University of Virginia. This work was funded by the National Institutes of Health (1RO1GM081576). 
Supplemental Figure 4-1: Circular dichroism and gel filtration of Hec1/Ndc80 CHD mutants. (A) CD spectra of $100 \mathrm{~nm}$ of the indicated $\mathrm{Ndc} 80^{\text {bonsai }}$ proteins were measured. Measurements were collected as described previously (Derewenda et al., 2007). (B) $\mathrm{Hec} 1^{\mathrm{WT}}, \mathrm{Hec}^{\Delta \mathrm{N}}, \mathrm{Hec}^{\mathrm{NEU}}$ and $\mathrm{Hec} 1^{\mathrm{K} 89 \mathrm{E} / \mathrm{K} 115 \mathrm{E}} \mathrm{Ndc} 80^{\text {bonsai }}$ proteins were fractionated over a Superdex 200 gel filtration column. Fractions spanning the length of the column run were separated on SDS-PAGE gels and viewed either by Coomassie stain $\left(\mathrm{Hec} 1^{\mathrm{WT}}\right)$ or Western blot $\left(\mathrm{Hec}^{\mathrm{K} 89 \mathrm{E} / \mathrm{K} 115 \mathrm{E}}, \mathrm{Hec}^{\Delta \mathrm{N}}\right.$ and $\left.\mathrm{Hec}^{\mathrm{NEU}}\right)$. 

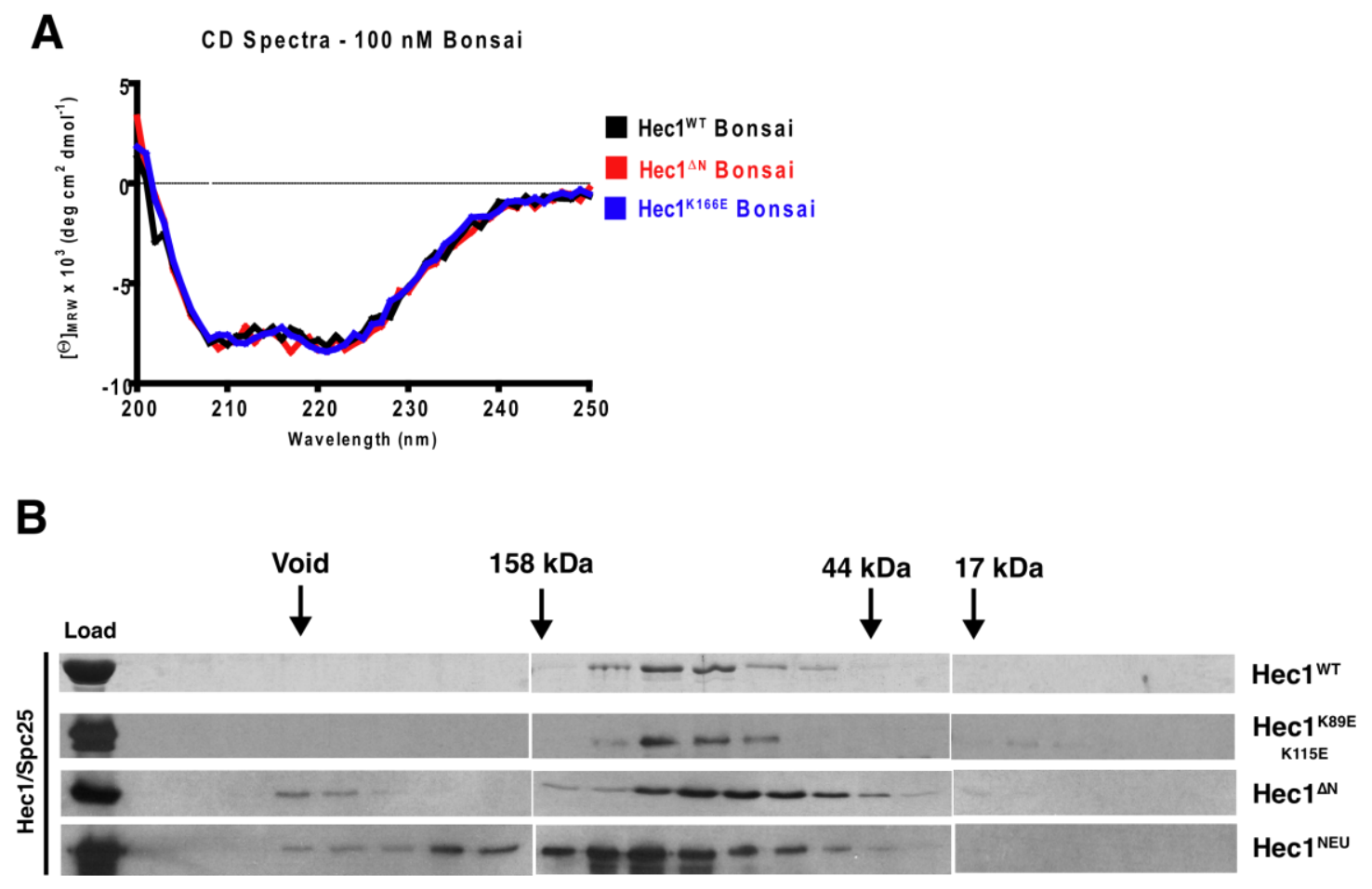

Supplemental Figure 4-1 
Supplemental Figure 4-2: Controls demonstrating effectiveness of Hec1/Ndc80 siRNA and replacement protocol

(A) Western blot for Hec1/Ndc80 (9G3 antibody) showing Hec1/Ndc80 protein levels for mock treated (GAPDH siRNA/ EGFP plasmid rescue), siHec1 (Hec1 siRNA/EGFP plasmid rescue), $\mathrm{Hec} 1^{\mathrm{K} 89 \mathrm{E} / \mathrm{K} 166 \mathrm{E}}$ and $\mathrm{Hec} 1^{\mathrm{NEU}}$ conditions at various time points in knockdown and replacement protocol. Exogenously expressed $\mathrm{Hec} 1 / \mathrm{Ndc} 80$ contains a EGFP tag and results in a slower-migrating form of the protein. (B) Metaphase aligned

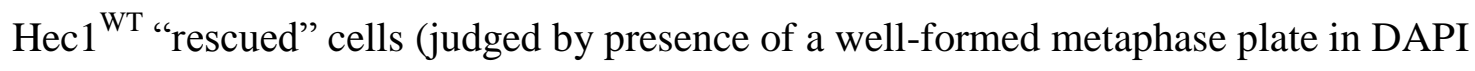
channel) were scored for expression or absence of GFP kinetochore dots in corresponding FITC channel. (C) Relative intensity levels of Hec1/Ndc80 immunostaining were quantified for mock treated, $\mathrm{Hec} 1^{\mathrm{WT}}$ and $\mathrm{Hec} 1^{\mathrm{K} 89 \mathrm{E} / \mathrm{K} 166 \mathrm{E}}$ cells. Ten kinetochores from ten cells $(\mathrm{N}=100)$ were quantified for each condition, and the mean intensity value was plotted. The mock condition was assigned an arbitrary value of 1 . 

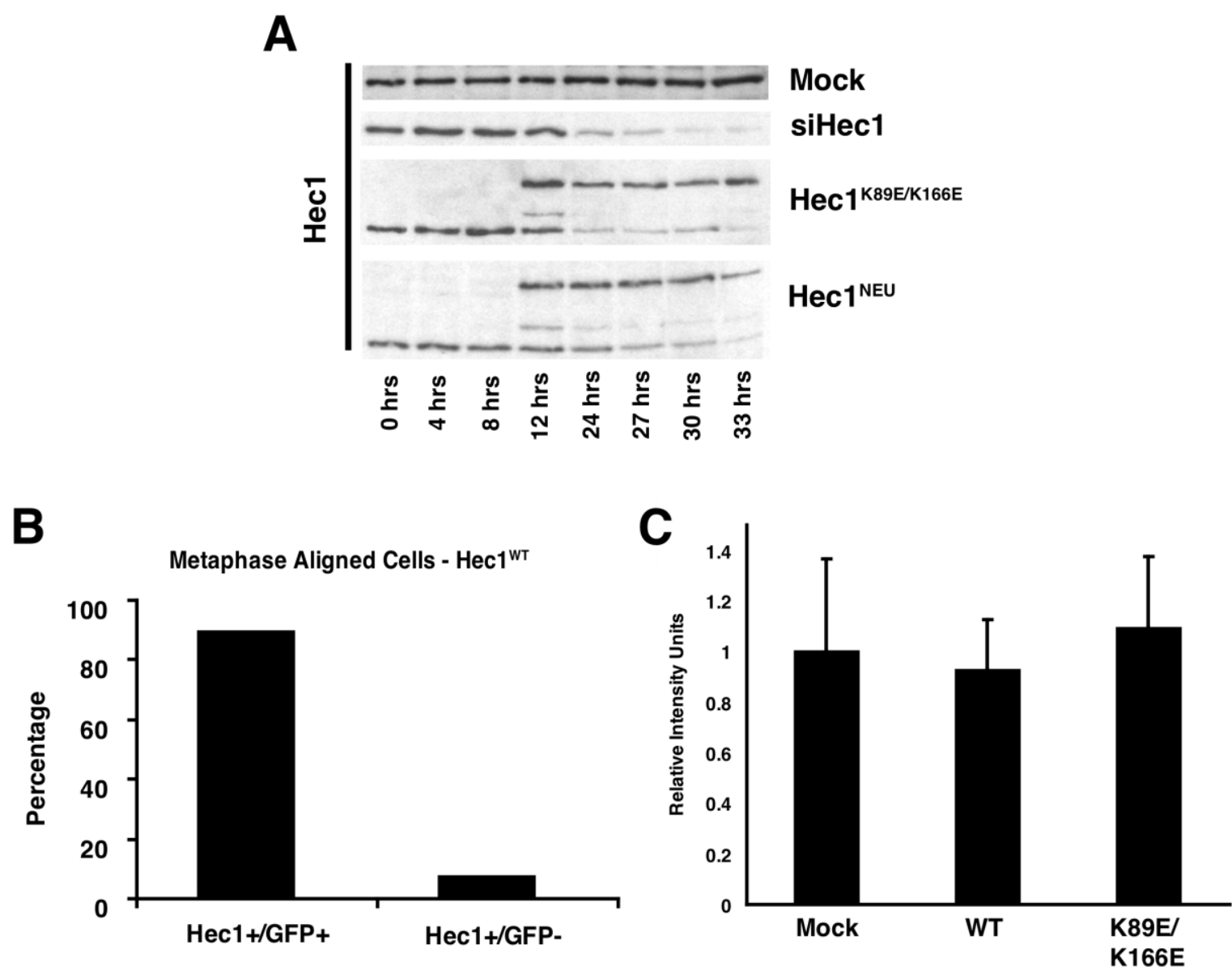

Supplemental Figure 4-2 
Supplemental Figure 4-3: Kinetochores assemble properly in Hec1/Ndc80 CHD mutant cells

After siRNA knockdown and replacement with the indicated Hec1/Ndc80 constructs (top labels) cells were fixed and immunostained for individual kinetochore proteins indicated on the left. Antibodies were chosen to select different regions of the kinetochore. ACA antibody reacts with centromeric antigens; KNL-1 (inner kinetochore), Hec1/Ndc80 and Spc25 (outer kinetochore) and CENP-E (fibrous corona) span the length of the prometaphase kinetochore. BubR1 is a spindle checkpoint protein. Representative cells are shown for each condition. 


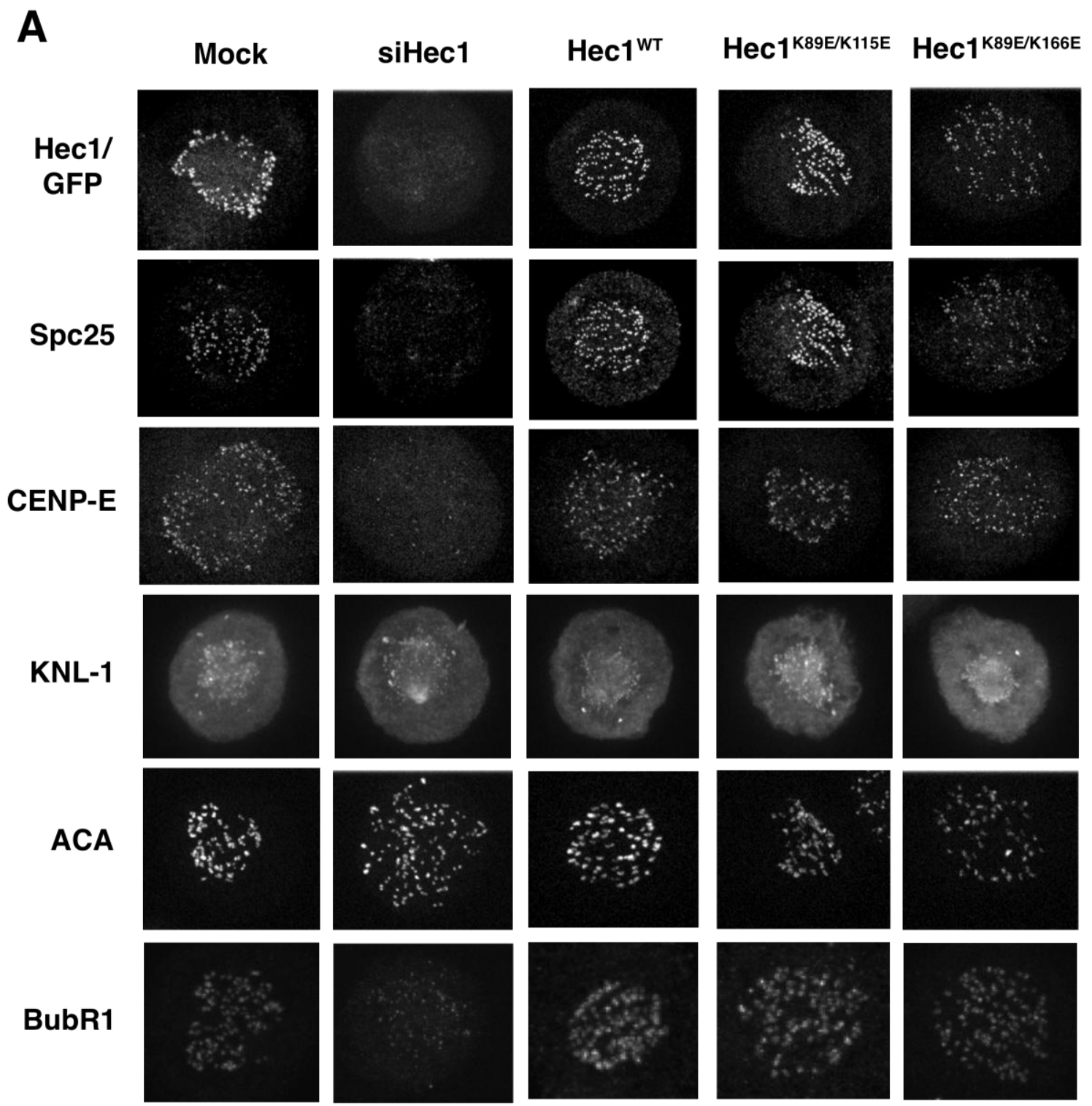

Supplemental Figure 4-3 
Supplemental Figure 4-4: Inter-kinetochore distance measurements

(A, B, C) Residues in the Hec1/Ndc80 CHD are critical for generating tension across sister kinetochores. Ten sister kinetochores in 10 cells $(\mathrm{N}=100)$ were identified by ACA staining and the distance between sisters was measured. Measurements for nocodazoletreated cells, $\mathrm{N}=1$; all other conditions $\mathrm{N}=3$. Error bars $=\mathrm{SD} .($ Noc $=$ Nocodazole $)$. 
A

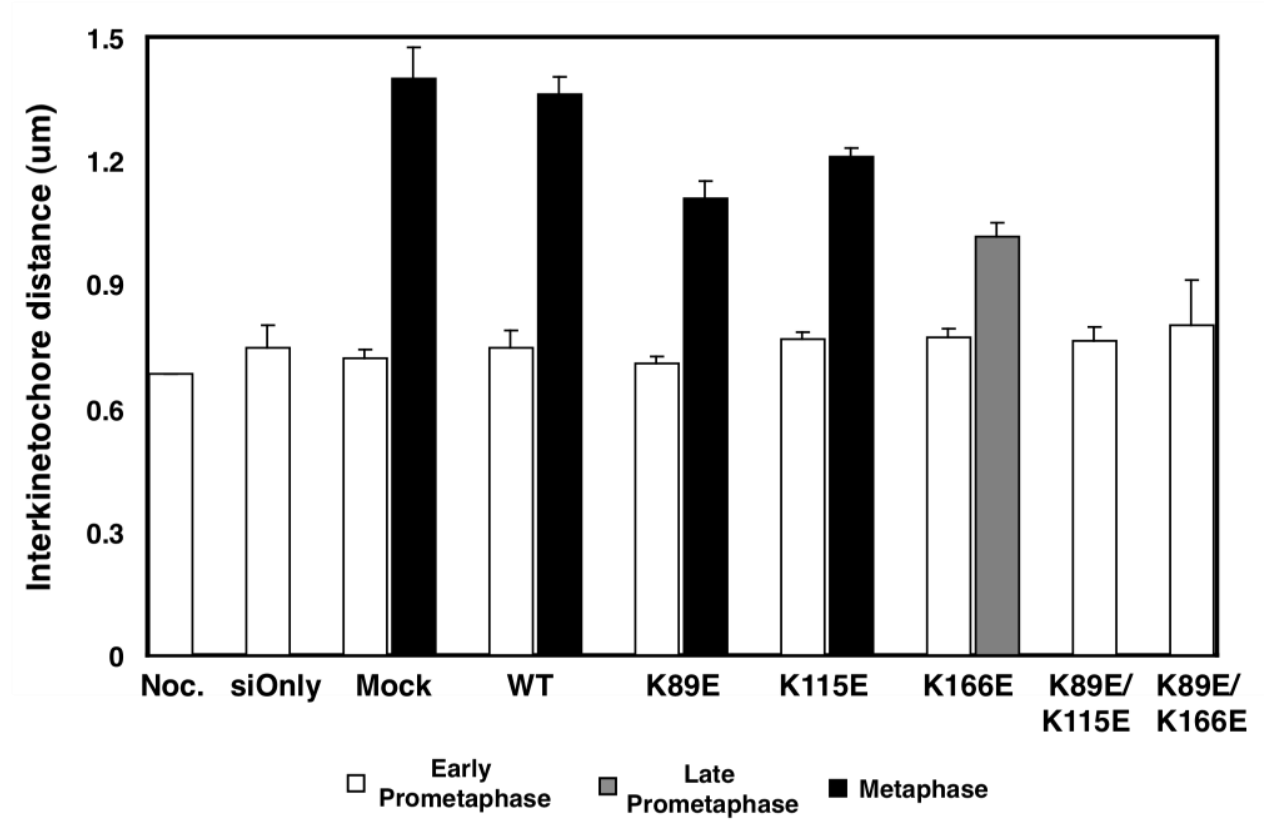

B

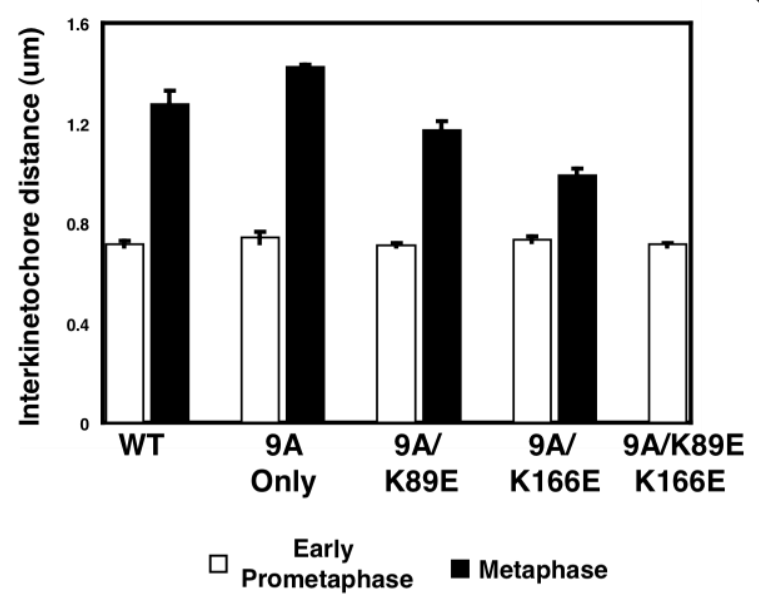

C

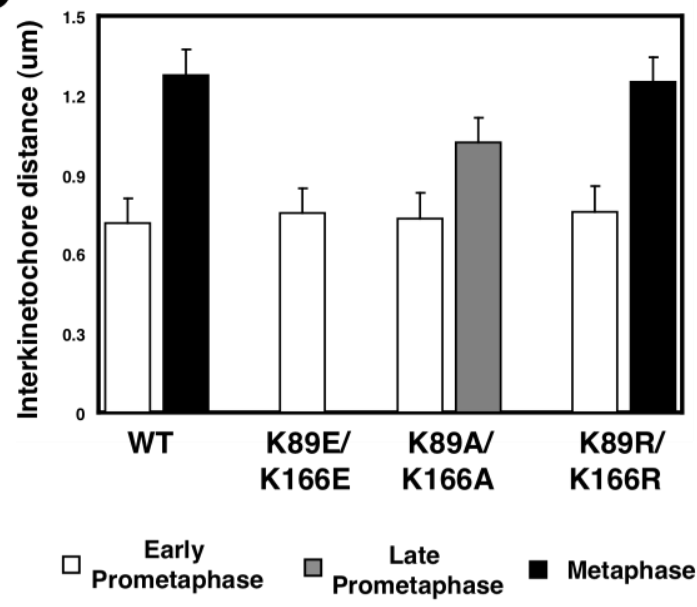

Supplemental Figure 4-4 
Supplemental Figure 4-5: Representative images of microtubules following cold treatment

(A) Cells were incubated in ice-cold media prior to fixation and immunostaining. Shown are representative images for cells scored as early prometaphase $\left(\mathrm{Hec} 1^{\mathrm{K} 89 \mathrm{E} / \mathrm{K} 166 \mathrm{E}}\right)$, late prometaphase $\left(\mathrm{Hec} 1^{\mathrm{K} 166 \mathrm{E}}\right)$ and metaphase $\left(\mathrm{Hec} 1^{\mathrm{WT}}\right.$, Hec $1^{\mathrm{K} 89 \mathrm{E}}$ and $\left.\mathrm{Hec} 1^{\mathrm{K} 115 \mathrm{E}}\right)$. For simplicity, only microtubules (red) and GFP-Hec1 (green) are shown. 
A

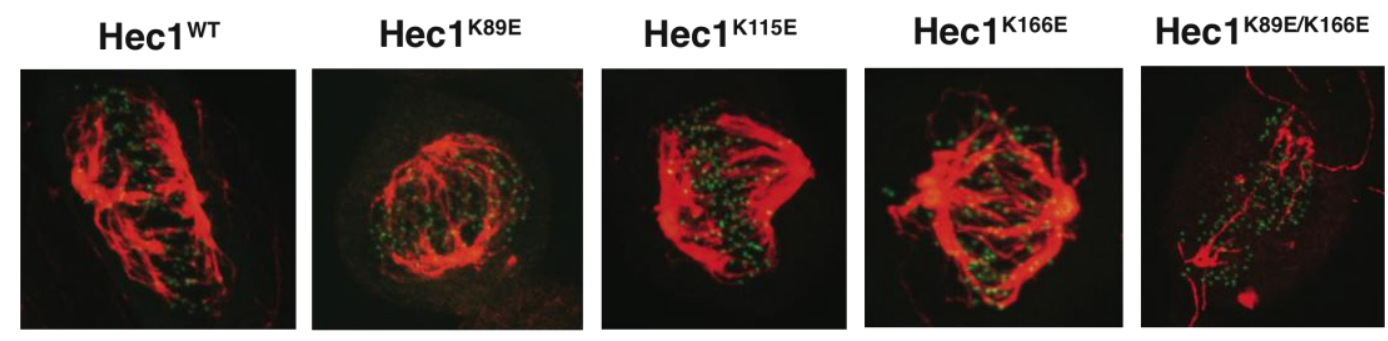

Supplemental Figure 4-5 
Supplemental Figure 4-6: Kinetochores assemble properly in Hec1 ${ }^{\text {NEU }}$ mutant cells

After siRNA knockdown and replacement with the indicated constructs (top labels) cells were fixed and immunostained for individual kinetochore proteins indicated on the left. Antibodies were chosen to select different regions of the kinetochore. ACA antibody reacts with centromeric antigens; Hec1/Ndc80 and Spc25 (outer kinetochore) and CENPE (fibrous corona) span the length of the prometaphase kinetochore. Mad2 is a spindle checkpoint protein. Representative cells are shown for each condition. 


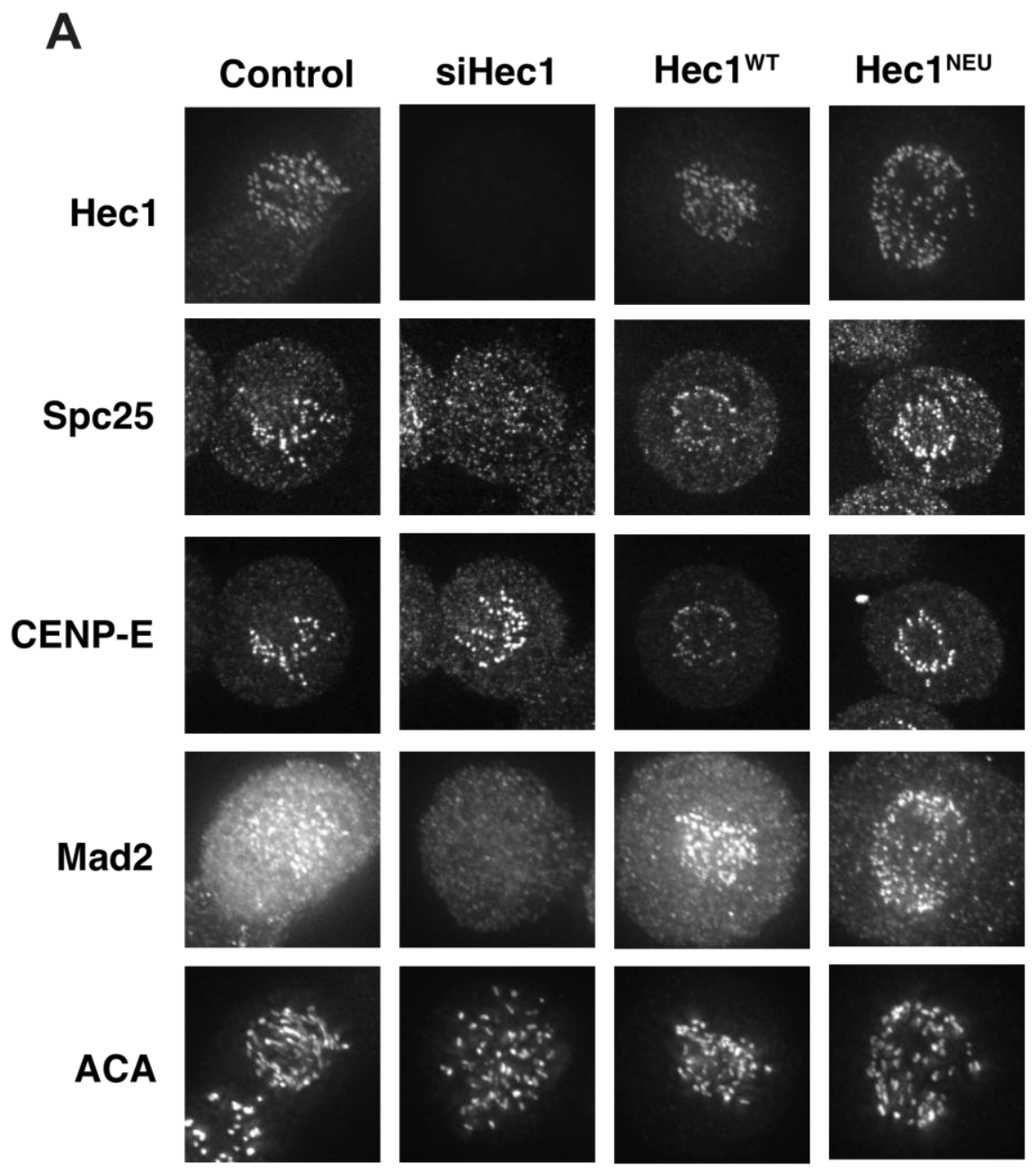

Supplemental Figure 4-6 


\section{CHAPTER 5: ANALYSIS OF HEC1/NDC80 TAIL FUNCTION - DISTINCT REGIONS IMPART UNIQUE IN VIVO FUNCTIONS}

This chapter is based on unpublished work. 


\section{Abstract}

Though the kinetochore contains more than 80 proteins, few are as critical for kinetochore function as the four-member Ndc80 complex. More specifically, the $\mathrm{Hec} 1 / \mathrm{Ndc} 80$ subunit is absolutely required for chromosome alignment in all eukaryotic systems studied. As the Ndc80 complex also plays critical roles in kinetochore assembly and spindle checkpoint signaling, it has proven necessary to generate separation-offunction mutants that allow more precise interpretation of Ndc80 phenotypes. We have previously demonstrated that both the $\mathrm{Hec} 1 / \mathrm{Ndc} 80 \mathrm{CHD}$ and the $\mathrm{N}$-terminal tail are critical for binding microtubules and congressing chromosomes in vivo. The CHD contacts microtubules at the tubulin dimer interface and is postulated to serve as a tubulin conformation sensor for the kinetochore. The function of the tail, in contrast, remains uncertain.

Here, we extend our studies of the Hec1/Ndc80 tail by examining two discrete sub-regions that exist within the tail. We find that impairment of the C-terminal region, which shows high affinity for microtubules in vitro, does not severely compromise chromosome alignment in vivo. Rather, a region at the N-terminus of the tail is required to align chromosomes into a metaphase plate. This region does not have a high affinity for microtubules but may be required for cooperative Ndc80 complex patch formation. This suggests that tight packing of Ndc80 complexes on a microtubule is critical for in vivo function. We propose a model whereby there are two interaction points in the vertebrate $\mathrm{Hec} 1 / \mathrm{Ndc} 80$ tail, with the $\mathrm{N}$-terminal region being the most critical for chromosome alignment. In the absence of either interaction point, the remaining region allows kinetochores to partially align chromosomes but not to satisfy the spindle 
checkpoint. We conclude this chapter by discussing new models for Ndc80 complex function in both humans and budding yeast and finish with a section detailing future directions to further define the mechanism of Ndc80 complex function. 


\section{Background}

Extensive study of the Ndc80 complex has begun to allow more precise functions to be assigned to discrete structural elements. The Hec1/Ndc80 CHD is required for conformation-dependant microtubule binding and may also play a role in checkpoint signaling (Alushin et al., 2010; Ciferri et al., 2008; Guimaraes et al., 2008; Sundin et al., 2011; Tooley et al., 2011), while the unstructured loop in the Hec1/Ndc80 coiled-coil region recruits other MAPs to the kinetochore and may permit complex bending (Hsu and Toda, 2011; Maure et al., 2011; Wang et al., 2008). Additionally, removal of either the Hec1 CHD or the unstructured loop is believed to create instability in the overall structure of the Ndc80 complex, thereby affecting kinetochore assembly.

Determining a precise role for the unstructured $\mathrm{Hec} 1 / \mathrm{Ndc} 80$ tail has proven more challenging. The tail is not required for kinetochore assembly (Miller et al., 2008) or SAC signaling (Guimaraes et al., 2008) but is absolutely required for in vitro microtubule binding in all model systems studied. In budding yeast a dimer of Ndc80 and Nuf 2 binds microtubules with a 10-fold lower affinity when the Ndc80 tail is removed, but wild type Ndc80 but cannot facilitate cooperative binding (Wei et al., 2007). Notably, the budding yeast Ndc80 tail is dispensable for viability (Kemmler et al., 2009; Lampert et al., 2010). In vertebrates, the tail is required both for microtubule binding in vitro and for chromosome alignment in vivo (Guimaraes et al., 2008; Miller et al., 2008; Tooley et al., 2011). The tail forms electrostatic interactions with the negatively charged tails of tubulin but also is required to generate patches of recombinant Ndc80 complexes on taxolstabilized microtubules (Alushin et al., 2010; Miller et al., 2008). Additionally, the tail contains nine predicted Aurora B phosphorylation sites and mutation of these sites 
impairs microtubule binding and patch formation in vitro as well as chromosome alignment in vivo (Alushin et al., 2010; Cheeseman et al., 2006; DeLuca et al., 2006; Guimaraes et al., 2008; Tooley et al., 2011; Welburn et al., 2010). Therefore, to determine why the Hec1/Ndc80 tail is required by vertebrate kinetochores, it is critical to discern the mechanism by which the tail generates attachments to microtubules and to determine how this process differs from budding yeast.

The human Hec1/Ndc80 tail contains 15 positively charged residues and five negatively charged residues for a net charge of +10 . Reducing the net charge of the tail to zero impairs microtubule binding in vitro and eliminates chromosome congression in vivo, demonstrating that large amounts of the charge on the tail are critical for kinetochore function (Tooley et al., 2011). Assuming that all nine predicted Aurora sites are bona fide in vivo targets, full phosphorylation of the tail could create a similar situation in a cell (net charge of +1 ) (Figure 5-1A). However, it remains uncertain whether full tail phosphorylation is required to correct attachment errors.

A closer examination of the tail reveals that the nine putative Aurora sites fall into two distinct regions where positive charge is clustered (Figure 5-1B). Serines 4, 5, 8 and 15 reside in the N-terminal "tip" region of the tail (N-Tip). Despite being positively charged, the N-Tip is unable to bind microtubules on its own (G. Alushin, unpublished data). Closer to the C-terminus of the tail, Threonine 49 and Serines 44, 55, 62 and 69 reside in a 25 -amino acid stretch of sequence. This $\mathrm{C}$-terminal region has recently been mapped as the microtubule-binding region for the Hec1/Ndc80 tail (G. Alushin, unpublished data) and we will therefore refer to this region as the microtubule-binding domain (MTBD). 
Preliminary in vitro data suggests that both the N-Tip and the MTBD can facilitate cooperative binding or patch formation (Alushin et al., 2010; Maiolica et al., 2007). However, it remains possible that cooperativity is simply an in vitro phenomenon and the Hec1/Ndc80 tail relies primarily on electrostatic interactions in vivo. Knowing that the MTBD has an affinity for microtubules but the N-Tip does not, we set out to determine which regions of the tail are most critical for creating tension-generating microtubule attachments and aligning chromosomes in vivo. We find that both tail regions contribute to full chromosome alignment but that the N-Tip is more critical. We demonstrate a requirement for the $\mathrm{N}$-Tip region in two ways, first by deleting this region from the tail and then by eliminating positive charge specifically in the N-Tip. These data extend our understanding of vertebrate $\mathrm{Hec} 1$ tail function and suggest that cooperative binding of Ndc80 complexes is a critical component of in vivo function. 
Figure 5-1: The Hec1 N-terminal tail is comprised of two basic regions that contain Aurora B regulatory sites

(A) $\mathrm{WT}=$ Schematic of full-length wild type Hec1 tail (+10 net charge). Positively charged residues are indicated by blue rectangles; negatively charged residues are indicated by red rectangles. Aurora B sites = nine putative Aurora B phosphorylation sites are indicated by purple rectangles. $\mathrm{P}-\mathrm{WT}=\mathrm{a}$ Hec1 tail phosphorylated on all nine putative Aurora B sites would contain a net charge of +1 . Note that Aurora sites reside in regions containing clusters of positive charge. (B) A closer examination of the tail reveals two distinct regions of high positive charge. The N-Tip region spans amino acids 1-35; the microtubule binding domain (MTDB) spans amino acids 40-69. Positively charged residues are indicated by blue rectangles; negatively charged residues are indicated by red rectangles. 
A

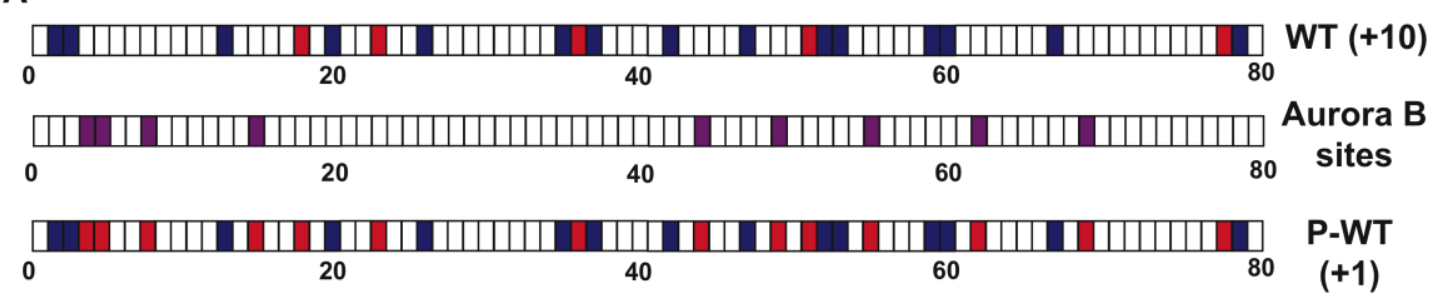

B

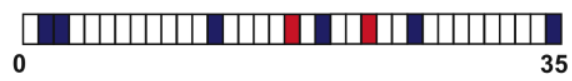
$\underset{40}{\mathrm{~W}}$

Positive Charge $\mathbf{n}$ Negative Charge $\mathbf{a u r o r a}$ B site

Figure 5-1 


\section{Materials and Methods}

\section{Recombinant protein cloning, expression, and purification}

The bicistronic plasmid that expresses human $\mathrm{Ndc} 80^{\text {Bonsai }}$ (a gift from A. Musacchio) was mutated to generate a series of N-terminal truncation mutants: $(\Delta 10, \Delta 20, \Delta 30, \Delta 40, \Delta 50$, $\Delta 60, \Delta 70$, and $\Delta 80(\Delta N))$. Wild type and mutant proteins were expressed and purified as previously described (Ciferri et al., 2008). Purified protein was dialyzed using a Slide-ALyzer Dialysis Unit (Thermo Fisher Scientific) into dilution buffer (100 mM KCl, $10 \mathrm{mM}$ Na-HEPES, $1 \mathrm{mM}$ EDTA, $1 \mathrm{mM}$ DTT, $1 \mathrm{mM} \mathrm{MgCl} 2,0.1 \mathrm{M} \mathrm{CaCl}_{2}, 10 \%$ glycerol (pH 7.5)).

\section{Microtubule polymerization and co-sedimentation assays}

Phospho-cellulose purified tubulin was polymerized as described ((Desai et al., 1999a). For all $\mathrm{Ndc} 80^{\text {Bonsai }}$ proteins, co-sedimentation assays were performed as described (Cheeseman et al., 2006) using a final concentration of $100 \mathrm{mM} \mathrm{Ndc80} 0^{\text {Bonsai }}$ and microtubule concentrations (tubulin monomer) as indicated. Western blotting and quantification of $\mathrm{Ndc} 80^{\text {Bonsai }}$ binding was performed as described (Tooley et al., 2011).

\section{Cloning of Hec1 constructs}

$\mathrm{Hec}^{+4 \mathrm{NT}}$ and Hec $1^{+4 \mathrm{CT}}$ mutants were generated by two-step PCR mutagenesis using the Hec $1^{\mathrm{NEU}}$ construct (Tooley et al., 2011) as a template. Hec $1^{+4 \mathrm{NT}}$ contains the following 6 residues mutated to alanine: $\mathrm{K} 2, \mathrm{R} 3, \mathrm{R} 13, \mathrm{R} 20, \mathrm{~K} 26$ and $\mathrm{K} 35$. $\mathrm{Hec} 1^{+4 \mathrm{CT}}$ contains the following 6 residues mutated to alanine: K42, K47, R52, K53, K59 and R60. The $\mathrm{Hec}^{\Delta 20-40}, \mathrm{Hec}^{\Delta 40-60}, \mathrm{Hec}^{\Delta 60-80}$, and $\mathrm{Hec}^{\Delta 40-80}$ mutants were generated by Splice 
Overlap Extension (SOE) PCR mutagenesis using the Hec1 ${ }^{\text {WT }}$ construct (Tooley et al., 2011) as a template. The $\mathrm{Hec} 1^{\Delta 20}$ and $\mathrm{Hec}^{\Delta 40}$ mutants were first generated in human $\mathrm{Ndc} 80^{\text {Bonsai }}$ and then sub-cloned into the wild type Hec1 rescue construct (Miller et al., 2008). All constructs generated were verified by sequencing.

\section{Cell culture and transfections}

HeLa cell culture, synchronization, and transfection conditions were performed essentially as described (Tooley et al., 2011). The Hec1 ${ }^{\Delta 20}$ and Hec1 ${ }^{\Delta 40}$ mutants were expressed in the FLAG-Hec1 rescue vector (Miller et al., 2008); all other rescued cells were expressed in the p-EGFP-N1 vector (Tooley et al., 2011). siRNA oligos targeted against Mad2 (Dharmacon) were transfected at a final concentration of $20 \mu \mathrm{M}$.

\section{Immunofluorescence}

Cell fixation conditions and immunofluorescence were performed as described (Tooley et al., 2011). Primary antibodies used were anti-Hec1 (1:500 [vol/vol]; GTX70268, GeneTex), anti-ACA (1:100 [vol/vol]; Antibodies Incorporated), anti-tubulin (1:500 [vol/vol]; NeoMarkers), FITC conjugated anti-tubulin (1:500 [vol/vol]; DM1 $\alpha$, Sigma) and anti-GFP (1:500 [vol/vol]). 


\section{Results}

\section{Binding properties of the Ndc80 complex are sensitive to changes in tail charge and length}

Removal of the entire Hec1/Ndc80 tail or elimination of its positive charge abolishes stable kinetochore-microtubule interactions and chromosome alignment in vivo (Guimaraes et al., 2008; Miller et al., 2008; Tooley et al., 2011). However, it is not known whether a more specific region of the tail is responsible for chromosome congression. To address this question we previously asked if there was a minimal tail region required for forming stable kinetochore-microtubule interactions. Our initial attempts to define a critical domain on the Hec1/Ndc80 tail involved truncating the tail from its $\mathrm{N}$-terminus in 10 amino acid increments. We created the following deletion set: $\Delta 10, \Delta 20, \Delta 30, \Delta 40, \Delta 50, \Delta 60, \Delta 70$, and $\Delta 80(\Delta \mathrm{N})$ Hec1 (Figure 5-2A). Microtubule pelleting experiments were performed using this deletion set expressed in the $\mathrm{Ndc} 80^{\text {Bonsai }}$ vector. We were unable to generate sufficient amounts of $\Delta 10, \Delta 20$ and $\Delta 30$ mutant protein for these pelleting assays, but we found that at high tubulin concentrations $(3 \mu \mathrm{M})$ $100 \mathrm{nM}$ of the $\Delta 40$ and $\Delta 50 \mathrm{Ndc} 80^{\text {Bonsai }}$ constructs bound microtubules as efficiently as wild type $\mathrm{Ndc} 80^{\text {Bonsai }}$ (S. Miller, unpublished data). These two mutant constructs were also capable of cooperative microtubule binding, suggesting that the first 50 amino acids of the tail are dispensable for generating wild type binding kinetics and cooperative microtubule binding. We also found that $\Delta 60 \mathrm{Ndc} 80^{\text {Bonsai }}$ bound microtubules poorly and lost cooperative binding. The $\Delta 70 \mathrm{Ndc} 80^{\text {Bonsai }}$ complex bound microtubules better than the $\Delta 60 \mathrm{Ndc} 80^{\text {Bonsai }}$ mutant, and at high tubulin concentrations ( $3 \mu \mathrm{M}$ tubulin) $\Delta 80$ Ndc $80^{\text {Bonsai }}$ bound microtubules at near wild-type levels (S. Miller, unpublished data). 
However, neither the $\Delta 70$ or $\Delta 80 \mathrm{Ndc} 80^{\text {Bonsai }}$ mutants bound microtubules cooperatively. These data suggested that a region located between amino acids 50-60 was required for cooperative microtubule binding. Indeed, generation of an $\mathrm{Ndc} 80^{\text {Bonsai }}$ mutant lacking this region $(\Delta 50-59)$ bound microtubules with wild-type binding kinetics but lost cooperative binding (S. Miller, unpublished data). The binding curves generated by the $\Delta 60, \Delta 70$, and $\Delta 80 \mathrm{Ndc} 80^{\text {Bonsai }}$ constructs also suggested that absent the first 59 amino acids of the tail, the 60-80 amino acid region was capable of inhibiting Ndc80 complex binding in vitro. Finally, the high levels of binding generated by $\Delta 80 \mathrm{Ndc} 80^{\text {Bonsai }}$ suggested that the tail was not required for microtubule binding when the dual CHDs were present and tubulin concentration was high. This final result contrasted with published MT binding data for Ndc $80^{\text {Bonsai }}$ or a dimer of the Hec1/Ndc80 and Nuf2 subunits, both of which showed a critical requirement for the tail in binding microtubules (Alushin et al., 2010; Ciferri et al., 2008; Wei et al., 2007).

Our binding experiments differed from published work in three key areas: the buffer composition, concentration of $\mathrm{Ndc} 80^{\text {Bonsai }}$ protein and the affinity tags used for protein purification. We were especially troubled by the inclusion of an extra 25 amino acid stretch of sequence (which included a 6-His tag) on the N-terminus of Hec1 in our $\mathrm{Ndc} 80^{\text {Bonsai }}$ constructs. There is precedent in the literature for placement of a tag on the N-terminus of Hec1 impairing Ndc80 complex function in vivo (Mattiuzzo et al., 2011). Therefore, we reasoned that our binding results might differ if the extraneous sequence no longer preceded the Hec1 tail in our Ndc80 ${ }^{\text {Bonsai }}$ constructs. We generated a new 10 amino acid tail deletion series in our $\mathrm{Ndc} 80^{\text {Bonsai }}$ constructs that lacked the 6-His tag and the extra 25 amino acids of sequence (Figure 5-2A). Proteins were expressed, purified 
(Figure 5-2B) and used in microtubule pelleting reactions that contained our previous buffer conditions and ligand concentration. We were unable to generate enough protein from the $\Delta 10 \mathrm{Ndc} 80^{\text {Bonsai }}$ construct to include this mutant in our analysis. Wild type Ndc $80^{\text {Bonsai }}$ achieved an average maximal binding of $58.2 \%$ bound at $2 \mu \mathrm{M}$ MTs. $\Delta 20$, $\Delta 30, \Delta 40$, and $\Delta 50$ achieved an average maximal binding of $63.9 \%, 69.8 \%, 43.1 \%$ and $54.7 \%$ bound, respectively (Figure 5-3A). The $\Delta 60$ complex was once again unable to effectively bind microtubules at all tubulin concentrations tested. The $\Delta 70$ complex also bound microtubules poorly in this set of experiments, only achieving a maximal binding of $5.6 \%$. The $\Delta 80$ complex - while unable to effectively bind microtubules at lower tubulin concentrations - reached a maximal binding of $52.5 \%$ bound at $4 \mu \mathrm{M}$ tubulin (Figure 5-3B). The variability of the data points for all conditions tested led to binding values with large standard deviations, and made it difficult to obtain accurate $\mathrm{K}_{\mathrm{d}}$ or Hill coefficient calculations. However, these experiments do confirm two previous findings: (1) the $\Delta 60 \mathrm{Ndc} 80^{\text {Bonsai }}$ complex is a poor microtubule binder and (2) the $\Delta 80 \mathrm{Ndc} 80^{\text {Bonsai }}$ complex can binding microtubules at high tubulin concentrations.

The charge neutral $(+0) \mathrm{Ndc} 80^{\text {Bonsai }}$ complex does not pellet in the absence of tubulin, but oligomerizes in the absence of microtubules (see Figure 4-4B and Supplemental Figure 4-1B). Additionally, other mutant $\mathrm{Ndc} 80^{\text {Bonsai }}$ complexes have been found to oligomerize/aggregate following protein purification (G. Alushin, personal communication). So although the $\Delta 60$ complex does not sediment in the absence of tubulin $(0 \mu \mathrm{M}$ tubulin condition, Figure 5-3B), we asked if this complex oligomerized in the absence of microtubules. To test this, we run purified $\Delta 60$ complex over a Superdex 200 gel filtration column and measured the elution profile of the protein complex as it 
exited from the column. While the majority of the complex exited the column at its predicted molecular weight, a sizable percentage of the protein also eluted from the column near the void volume (Figure 5-3C). This suggests that while the complex cannot sediment in the absence of microtubules, the $\Delta 60 \mathrm{Ndc} 80^{\text {Bonsai }}$ protein used in our MT pelleting assays contains Ndc80 complexes in varied states of oligomerization. The significance of this discrepancy in oligomerization states remains unclear. 
Figure 5-2: Purification of Hec1 tail truncation mutants in Ndc80 ${ }^{\text {Bonsai }}$

(A) Cartoon showing the Hec1 tail truncation mutants that were created for in vitro microtubule pelleting assays. $\mathrm{CHD}=$ Calponin Homology Domain. (B) Hec1 tail mutants that had been cloned into $\mathrm{Ndc} 80^{\text {Bonsai }}$ construct were expressed and purified. Coomassiestained gel shows the Hec1/Spc25 fusion protein (top band) and the Nuf2/Spc24 fusion protein (lower band) for the indicated $\mathrm{Ndc} 80^{\text {Bonsai }}$ constructs. $\mathrm{M}=$ marker. 
A

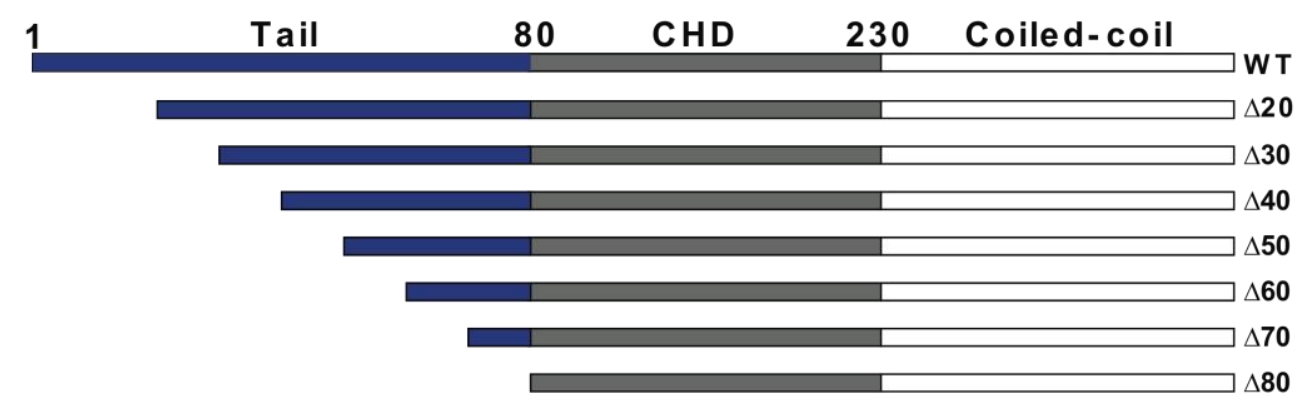

B

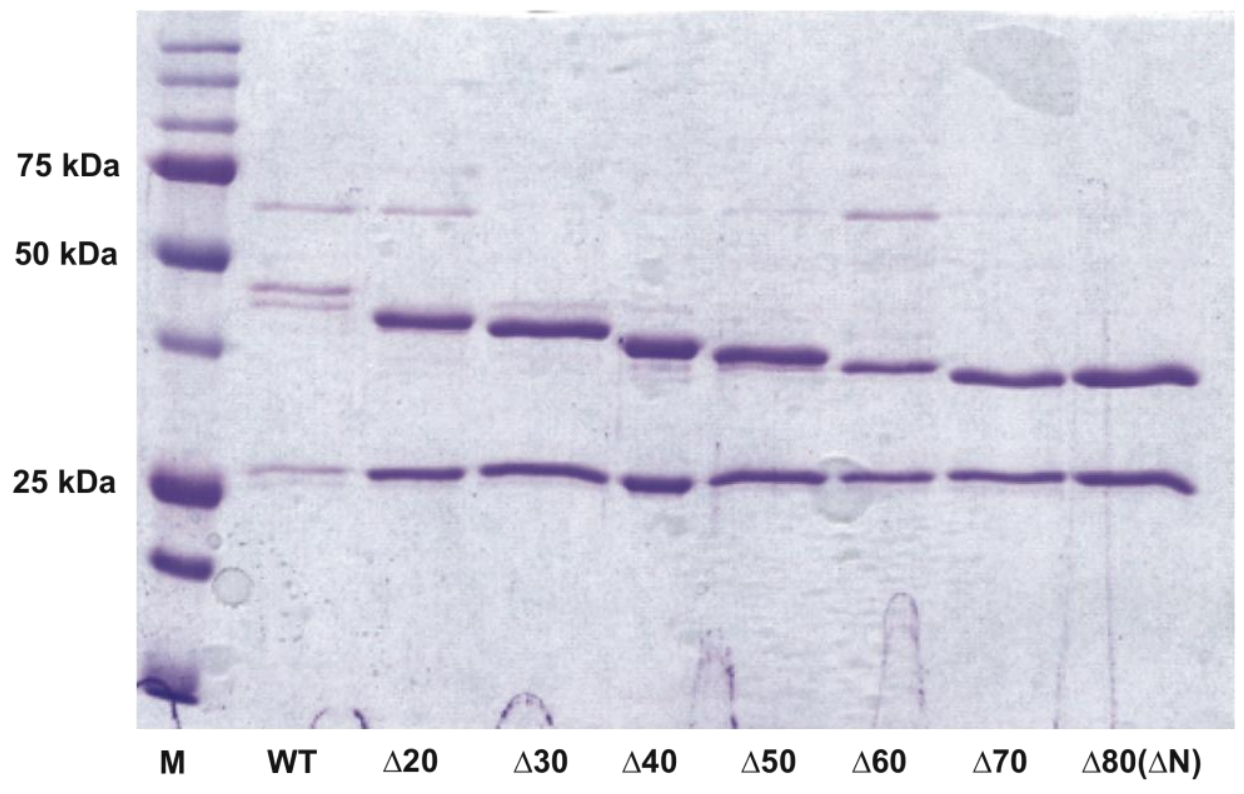

Figure 5-2 
Figure 5-3: Microtubule binding curves for $N d c 80^{\text {Bonsai }}$ tail mutants

(A) Microtubule binding curves for the wild type (WT), $\Delta 20, \Delta 30, \Delta 40$, and $\Delta 50$ Ndc $80^{\text {Bonsai }}$ constructs. $100 \mathrm{nM}$ of $\mathrm{Ndc} 80^{\text {Bonsai }}$ protein was incubated with the indicated amounts of taxol-stabilized microtubules for 10 minutes at room temperature before being spun through a glycerol cushion. Pellet samples $(\mathrm{N}=3)$ were subjected to Western blotting with an anti-Spc25 antibody. Signal intensities were quantified, and the mean intensity was plotted. (B) Binding curves for the WT, $\Delta 50, \Delta 60, \Delta 70$, and $\Delta 80(\Delta \mathrm{N})$ $\mathrm{Ndc} 80^{\text {Bonsai }}$ constructs. $100 \mathrm{nM}$ of $\mathrm{Ndc} 80^{\text {Bonsai }}$ protein was incubated with the indicated amounts of taxol-stabilized microtubules for 10 minutes at room temperature before being spun through a glycerol cushion. Pellet samples $(\mathrm{N}=3)$ were subjected to Western blotting with an anti-Spc25 antibody. Signal intensities were quantified, and the mean intensity was plotted. Error bars $=\mathrm{SD}$. (C) Purified $\Delta 60 \mathrm{Ndc} 80^{\text {Bonsai }}$ was run over a Superdex 200 column and $0.5 \mathrm{~mL}$ fractions were collected as the protein exited the column. Western blotting was performed on the indicated fractions using an anti-Spc25 antibody. $\operatorname{Load}=2 \mu \mathrm{g}$ recombinant protein. Void volume $=$ Fractions $4-7$. Ndc80 Bonsai M.W. = predicted molecular weight for Ndc80 ${ }^{\text {Bonsai }}$, Fractions 17-21. Vertical black line denotes break between Fractions 10 and 15 . 
A Bonsai deletion set

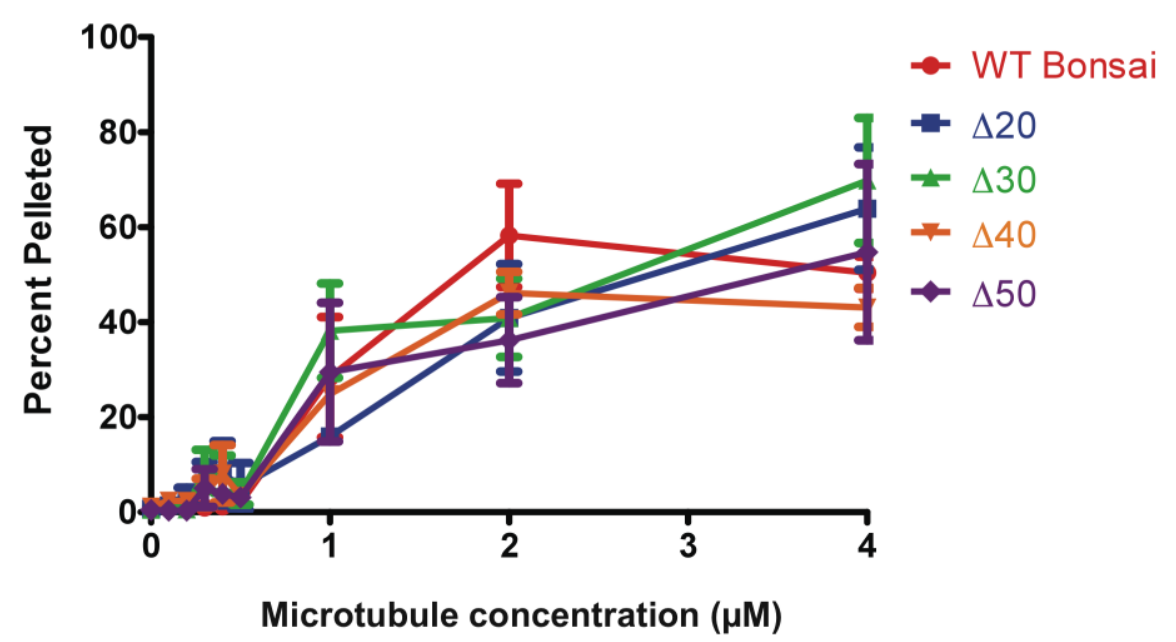

B

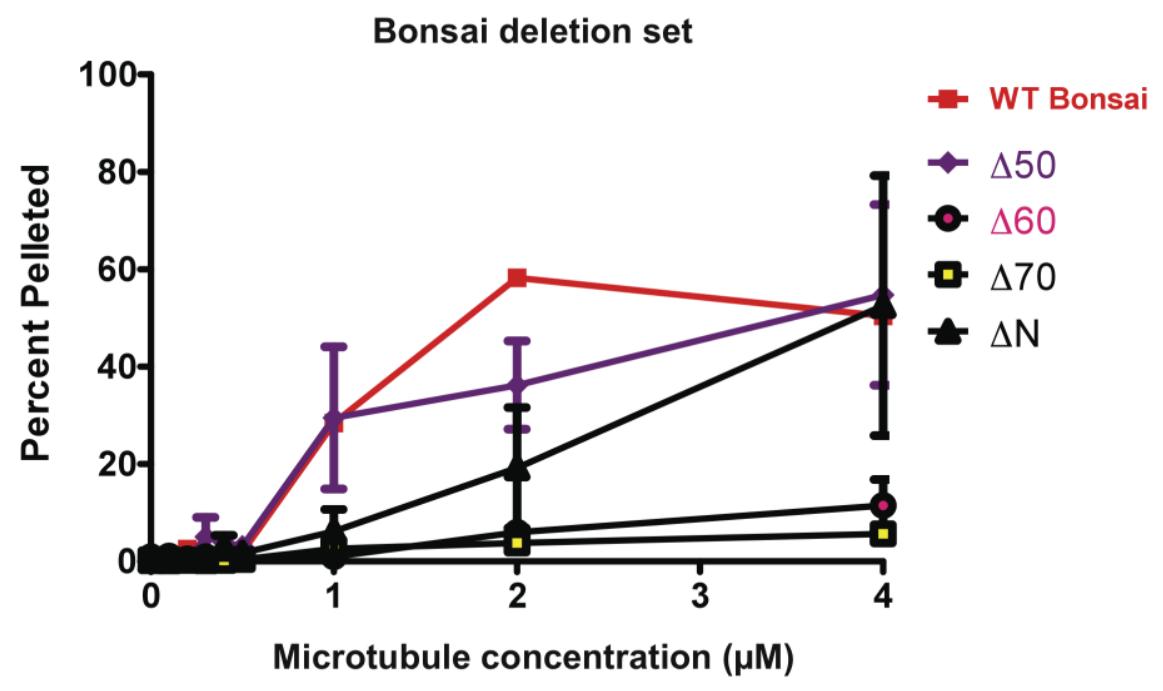

C

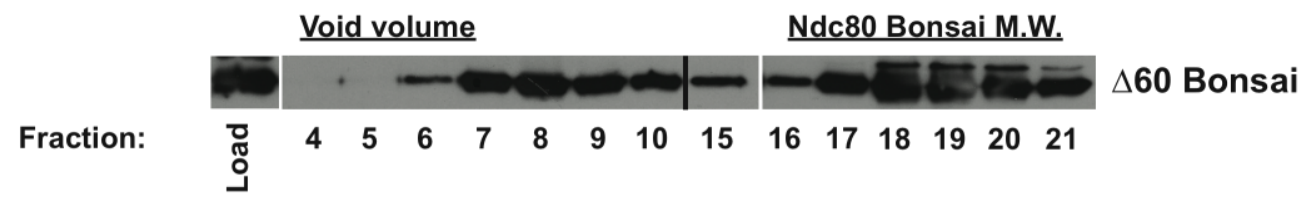

Figure 5-3 


\section{Minimal tail requirements for chromosome congression in vivo}

Given the conflicting data generated by our in vitro pelleting experiments, we next turned to our knockdown and rescue protocol in HeLa cells. We began by engineering the 10 amino acid tail deletion series into our rescue plasmids. Following knockdown of endogenous Hec1/Ndc80 and replacement with a plasmid encoding an siRNA-resistant Hec1 gene, we found that cells rescued with our Hec1 ${ }^{\Delta 10}, \mathrm{Hec}^{\Delta 20}$, and Hec $1^{\Delta 30}$ mutants were able to align chromosomes nearly as well as Hec1 ${ }^{\text {WT }}$ cells (S. Miller, unpublished data). This result was repeated in two independent experiments. Additionally, in both experiments the $\mathrm{Hec} 1^{\Delta 70}$ mutant was unable to align chromosomes into a metaphase plate, as had previously been seen for the $\mathrm{Hec}^{\Delta 80}$ mutant (Guimaraes et al., 2008; Miller et al., 2008).

Results for the Hec1 $1^{\Delta 40}$, Hec1 $1^{\Delta 50}$, and Hec $1^{\Delta 60}$ mutants differed between the two experiments. In one experiment, cells rescued with the Hec $1^{\Delta 40}$ mutant were capable of aligning chromosomes into a metaphase plate $50 \%$ less frequently control cells. The $\mathrm{Hec} 1^{\Delta 50}$ and Hec1 ${ }^{\Delta 60}$ mutant phenotypes largely mirrored the Hec $1^{\Delta 40}$ phenotype. In the second experiment the $\mathrm{Hec}^{\Delta 40}$ mutant aligned chromosomes in a greater percentage of cells (22\% of mitotic cells compared to $32 \%$ for control cells), and it was not until the tail was truncated back 60 amino acids from the $\mathrm{N}$-terminus that alignment ability began to sharply drop (S. Miller, unpublished data).

The data from these two preliminary experiments suggested that amino acids 4060 are critical for generating chromosome alignment in cells, in agreement with our in vitro data. However, an alternative interpretation of these data is that the $\mathrm{Hec}^{\Delta 40}$, $\mathrm{Hec}^{\Delta 50}$, and $\mathrm{Hec}^{\Delta 60}$ mutant kinetochores increasingly lose the ability to align 
chromosomes because - in addition to progressively losing tail length - the net positive charge on the tail has dropped below a threshold required for generating productive microtubule attachments. Indeed, the net charge of the tail in our Hec $1^{\Delta 40}$ mutant is +6 but the net charge drops to +4 in the $\mathrm{Hec}^{\Delta 50}$ mutant and +1 in the $\mathrm{Hec}^{\Delta 60}$ mutant. Therefore, we needed to generate a new set of tail mutants to distinguish between these two possibilities. The sharing of unpublished data regarding N-Tip and MTBD microtubule binding by the Nogales laboratory greatly aided the design of our new mutants.

We generated a deletion set that removed 20 amino acids from consecutive segments of the tail while leaving the other 60 amino acids intact to maintain a constant tail length (Figure 5-4A). This deletion set contains the Hec1 ${ }^{\Delta 40-60}$ mutant, which our previous data suggests is the critical region on the Hec1/Ndc80 tail and the Nogales lab has identified as the MTBD. We used our knockdown and rescue protocol to assay kinetochore function during the first mitosis during which rescued cells contain mutated Hec1/Ndc80 proteins. We first tested whether kinetochores containing these Hec1/Ndc80 tail mutants could properly align chromosomes into a metaphase plate. Metaphase was defined as the majority of cells in the center of the cell, and cells containing more than five unaligned chromosomes were scored as prometaphase. Cells were fixed 8-10 hours following release from thymidine block, when a majority of the cell population was traversing mitosis. Cells were stained for tubulin and DNA. Mock transfected and Hec1/Ndc80 knockdown cells were also stained with anti-Hec1/Ndc80 antibodies, while Hec1/Ndc80 in rescued cells was detected with anti-GFP antibodies. 
In mock transfected cells, $19.1 \pm 1.3 \%$ of mitotic cells were able to achieve proper chromosome alignment at the time of fixation (Figures 5-4B and 5-4C). Similarly, $16.7 \pm$ $2.5 \%$ of mitotic $\mathrm{Hec}^{\mathrm{WT}}$ cells had chromosomes aligned into a metaphase plate. $\mathrm{Hec} 1 / \mathrm{Ndc} 80$ knockdown, $\mathrm{Hec}^{\Delta \mathrm{N}}$ and $\mathrm{Hec}^{+0}$ conditions all contained less than $3 \%$ of mitotic cells with chromosomes aligned into a metaphase plate, consistent with published data (Guimaraes et al., 2008; Miller et al., 2008; Tooley et al., 2011). Surprisingly, replacement of endogenous $\mathrm{Hec} 1 / \mathrm{Ndc} 80$ with $\mathrm{Hec}^{\Delta 40-60}$ only resulted in a modest chromosome alignment phenotype, with $17.7 \pm 2.1 \%$ of $\mathrm{Hec}^{\triangle 40-60}$ cells aligning chromosomes into a metaphase plate. Similarly, between $13-17 \%$ of cells rescued with the Hec1 ${ }^{\Delta 20}$, Hec1 ${ }^{\Delta 20-40}$ or Hec1 ${ }^{\Delta 60-80}$ mutants aligned chromosomes into a metaphase plate. This indicates that while loss of any one of these regions renders kinetochores unable to align chromosomes as efficiently as wild type kinetochores, no one region of the tail is essential for aligning chromosomes.

A Hec1 tail deletion that removed half of the tail length generated a more severe alignment phenotype. However, while this $\operatorname{Hec}^{\Delta 40}$ mutant exhibits reduced alignment ability (11.0 $\pm 1.7 \%$ of mitotic cells contained metaphase aligned chromosomes) the $\mathrm{Hec}^{\Delta 40-80}$ mutant aligns chromosomes nearly as well as wild type cells $(17.0 \pm 3.5 \%$ of $\mathrm{Hec}^{\Delta 40-80}$ mitotic cells contained aligned chromosomes). These data suggest that the Nterminus of the tail is more critical for aligning chromosomes, though in its absence the C-terminal region is capable of providing some functional redundancy. 
Figure 5-4: The N-terminal region of the Hec1 tail is required for chromosome alignment

(A) Cartoon depicting the wild type Hec1 tail and the tail truncation mutants used for these studies. Positively charged residues are indicated by blue rectangles; negatively charged residues are indicated by red rectangles. (B) Representative images of the predominant mitotic figures from siHec1 knockdown and rescued cells that have been stained for Hec1/Ndc80 (green), tubulin (red) and ACA (blue). WT and most Hec1 tail mutant cells are identified by an expressed EGFP, and are co-stained for tubulin and DNA. Cells rescued with FLAG-Hec1 ${ }^{\Delta 20}$ and FLAG-Hec $1^{\Delta 40}$ are stained for Hec1/Ndc80 and contrast-enhanced to reduce background signal. (C) Mitotic cells were scored for chromosome alignment into a metaphase plate, and the percentage of metaphase cells was plotted. Metaphase cells were further subdivided to indicate cells with all chromosomes aligned (black), cells with 1-2 unaligned chromosomes (gray) and cells with 3-5 unaligned chromosomes (white). One hundred mitotic cells counted per experiment. 


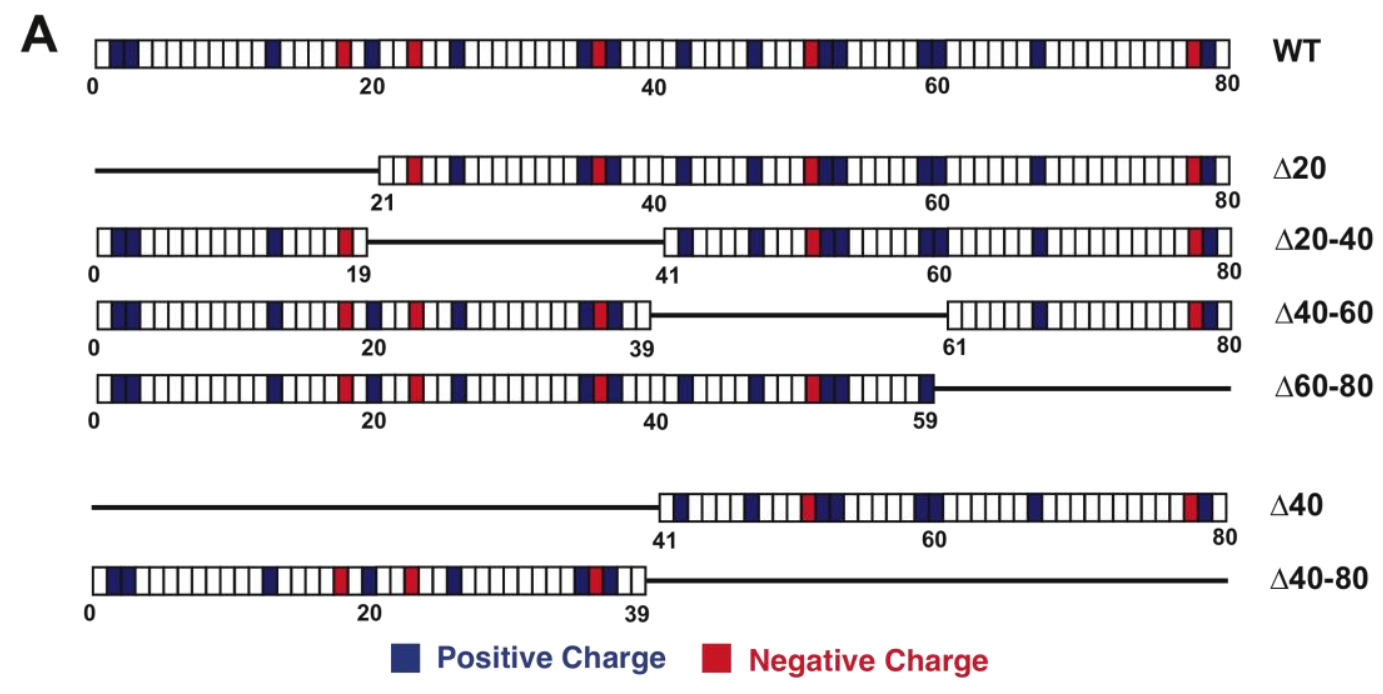

B
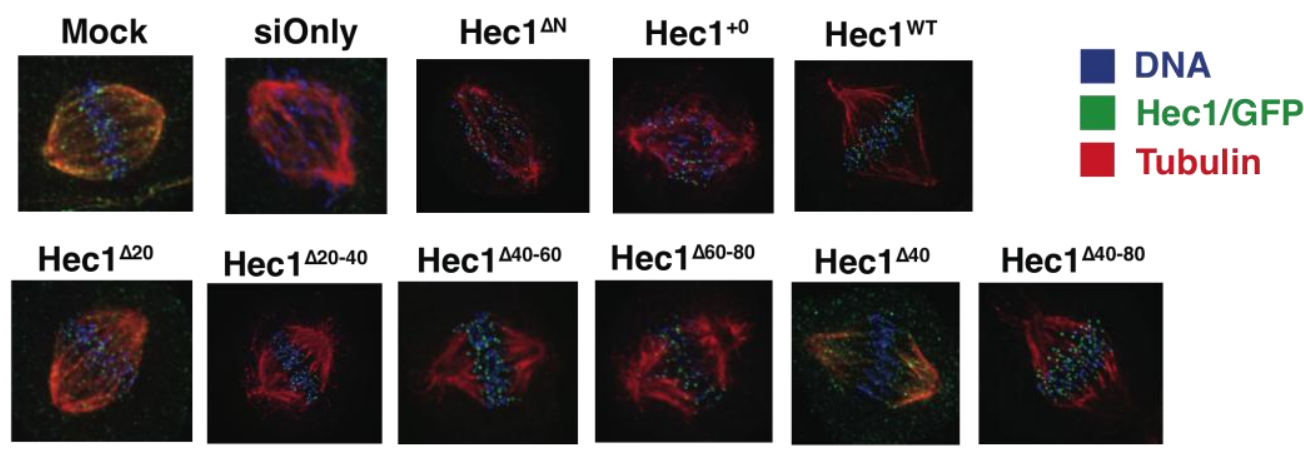

C

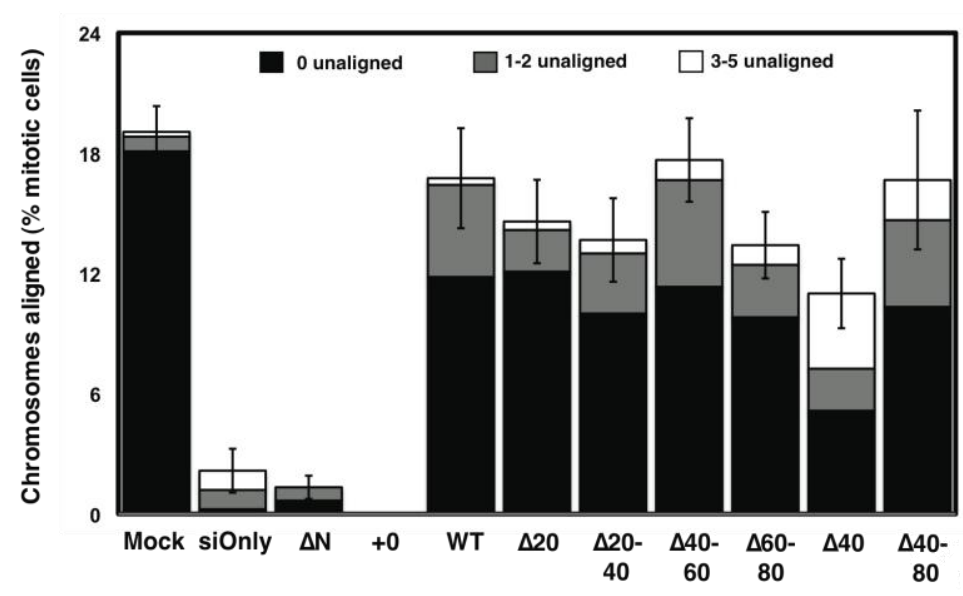

Figure 5-4 
To further distinguish between the $\mathrm{N}$ - and $\mathrm{C}$-terminal regions of the tail we generated a pair of mutants that maintained the 80 amino acid tail length but carried a reduced net charge of +4 . The lysine/arginine-to-alanine mutations that lowered the net charge were engineered into the two distinct tail regions. One mutant, $\mathrm{Hec} 1^{+4 \mathrm{NT}}$, targeted lysines and arginines located in the first 35 amino acids of the tail (the N-Tip). A second mutant, $\mathrm{Hec}^{+4 \mathrm{CT}}$, mutated residues in the 40-60 region (the MTBD) (Figure 5-5A). Replacement of endogenous $\mathrm{Hec} 1 / \mathrm{Ndc} 80$ with $\mathrm{Hec} 1^{+4 \mathrm{NT}}$ results in a chromosome alignment phenotype that is similar to what is seen for Hec1/Ndc80 knockdown, Hec1 ${ }^{\Delta \mathrm{N}}$ and $\mathrm{Hec}^{+0}$ cells (Figures 5-5B and 5-5C). Only $1.7 \pm 0.6 \%$ of $\mathrm{Hec}^{+4 \mathrm{NT}}$ mitotic cells align chromosomes into a metaphase plate, although some bipolar attachments are formed. In contrast, $12.0 \pm$ $5.0 \%$ of $\mathrm{Hec}^{+4 \mathrm{CT}}$ cells align chromosomes into a metaphase plate - though there is an increase in metaphase cells with some unaligned chromosomes. Therefore, despite carrying the same net charge, these two mutants further demonstrate that there are subdomains within the tail with different requirements for chromosome alignment. It remains uncertain why the $\mathrm{Hec}^{+4 \mathrm{NT}}$ phenotype is more severe than the $\mathrm{Hec}^{\Delta 40}$ phenotype, and potential reasons for this discrepancy will be discussed later in this chapter.

While the majority of our Hec1 tail mutants are capable of congressing chromosomes, almost all of these alignment-capable mutants are unable to progress into anaphase. This is not due to cell fixation occurring at too early a time point, as $5.0 \pm 0.7 \%$ of mitotic Hec $1^{\text {WT }}$ cells are observed in anaphase (mock treated cells also exhibit numerous anaphase figures) (Figure 5-6A). Notably, we observed $13.0 \pm 4.6 \%$ of $\mathrm{Hec}^{\Delta 60-80}$ cells and $2.0 \pm 1.7 \%$ of $\mathrm{Hec}^{\Delta 20-40}$ cells in anaphase, which argues that a fulllength tail is not required for satisfying the spindle checkpoint. 
We next asked why cells rescued with these Hec1 tail mutants - despite aligning chromosomes into a metaphase plate - are incapable of progressing into anaphase. We began by asking if cells could not enter anaphase due to SAC activation. To test this, we performed knockdown and rescue experiments where both Hecl and the spindle checkpoint protein Mad2 were knocked down concurrently. Following co-knockdown of endogenous Hec1 and $\mathrm{Mad} 2$ and rescue with Hec1 proteins containing mutations in both regions of the tail, we find that cells are now capable of progressing into anaphase despite being fixed at the same time point as our previous experiment (Figure 5-6B). These preliminary data suggest that our Hec1 tail mutants are arresting in a SAC-dependant manner.

We next asked what attachment defect was responsible for generating the spindle checkpoint arrest. One obvious explanation for SAC activation would a lack of tension normally generated on kinetochores by microtubule pulling forces. Tension on kinetochores can be measured by calculating the distance between sister kinetochores (inter-kinetochore distance). This distance measurement can be used as a readout of the forces exerted on kinetochores by microtubules. Cells treated with nocodazole lack spindle microtubules and hence pulling forces cannot by exerted on kinetochores. Sister kinetochores from nocodazole-treated cells have inter-kinetochore distance measurements of $0.74 \mu \mathrm{m}$. Similarly, cells in early prometaphase have not yet generated productive microtubule attachments and kinetochores in these cells do not come under appreciable tension (Figure 5-5D). Hec1 ${ }^{\mathrm{WT}}$ cells in metaphase have inter-kinetochore distance measurements of $1.23 \pm 0.02 \mu \mathrm{m}$, while Hec1/Ndc80 knockdown cells cannot form productive attachments to microtubules and have inter-kinetochore distance 
measurements of $0.77 \pm 0.01 \mu \mathrm{m}$. Hec $1^{+4 \mathrm{NT}}$ cells in early prometaphase have similarly low inter-kinetochore measurements, but a population of $\mathrm{Hec} 1^{+4 \mathrm{NT}}$ cells did progress into late prometaphase at the time of fixation. Average inter-kinetochore distance measurements from these late prometaphase cells are $1.00 \pm 0.02 \mu \mathrm{m}$, demonstrating that kinetochores containing this mutant are capable of separating sisters but at a reduced level that likely would activate the checkpoint. In contrast, $\mathrm{Hec} 1^{+4 \mathrm{CT}}$ cells in metaphase were able to separate sister kinetochores to near wild type levels $(1.19 \pm 0.04 \mu \mathrm{m})$, as were $\operatorname{Hec}^{\Delta 20-40}(1.15 \pm 0.05 \mu \mathrm{m})$ and Hec1 ${ }^{\Delta 40-60}(1.19 \pm 0.02 \mu \mathrm{m})$ metaphase cells. This suggests that the spindle checkpoint arrest triggered by most of our Hec1 tail mutants is not due to a gross reduction in pulling forces on kinetochores. However, as even a single improperly attached kinetochore is capable of triggering the spindle checkpoint ( $\mathrm{Li}$ and Nicklas, 1995), we cannot rule out the possibility of a more subtle tension defect that was not captured in our analysis. Furthermore, other potential reasons exist for checkpoint arrest in situations where chromosomes align and tension is generated on kinetochores. These include delays in aligning all chromosomes, as well as a failure to maintain the integrity of the metaphase plate once it has formed (Daum et al., 2011; Sundin et al., 2011). Testing these possibilities remains an area for future research (see below). 
Figure 5-5: Distinct regions of the Hec1 tail have different alignment phenotypes

(A) Cartoon depicting the wild type Hec1 tail and tail mutants used for knockdown and rescue studies. Also depicted are the N-Tip and MTDB regions of the tail to highlight areas where mutants were engineered. Positively charged residues are indicated by blue rectangles; negatively charged residues are indicated by red rectangles. (B)

Representative images of the predominant mitotic figures from siHec1 knockdown and rescued cells that have been stained for Hec1/Ndc80 (green), tubulin (red) and ACA (blue). WT and Hec1 tail mutant cells are identified by an expressed EGFP, and are costained for tubulin and ACA. (C) Mitotic cells were scored for chromosome alignment into a metaphase plate, and the percentage of metaphase cells was plotted. Metaphase cells were further subdivided to indicate cells with all chromosomes aligned (black), cells with 1-2 unaligned chromosomes (gray) and cells with 3-5 unaligned chromosomes (white). One hundred mitotic cells counted per experiment. (D) Ten sister kinetochores in at least five cells $(\mathrm{N}>50)$ were identified by ACA staining between Hec1/Ndc80 signals, and the distance between those sister kinetochores was measured $(\mathrm{N}=3)$. The mean distance is plotted for early prometaphase (gray) and metaphase (black) cells. For the $\mathrm{Hec}^{+4 \mathrm{NT}}$ mutant, measurements for late prometaphase cells are represented in black. Noc. $=$ nocodazole $($ white $)$. 


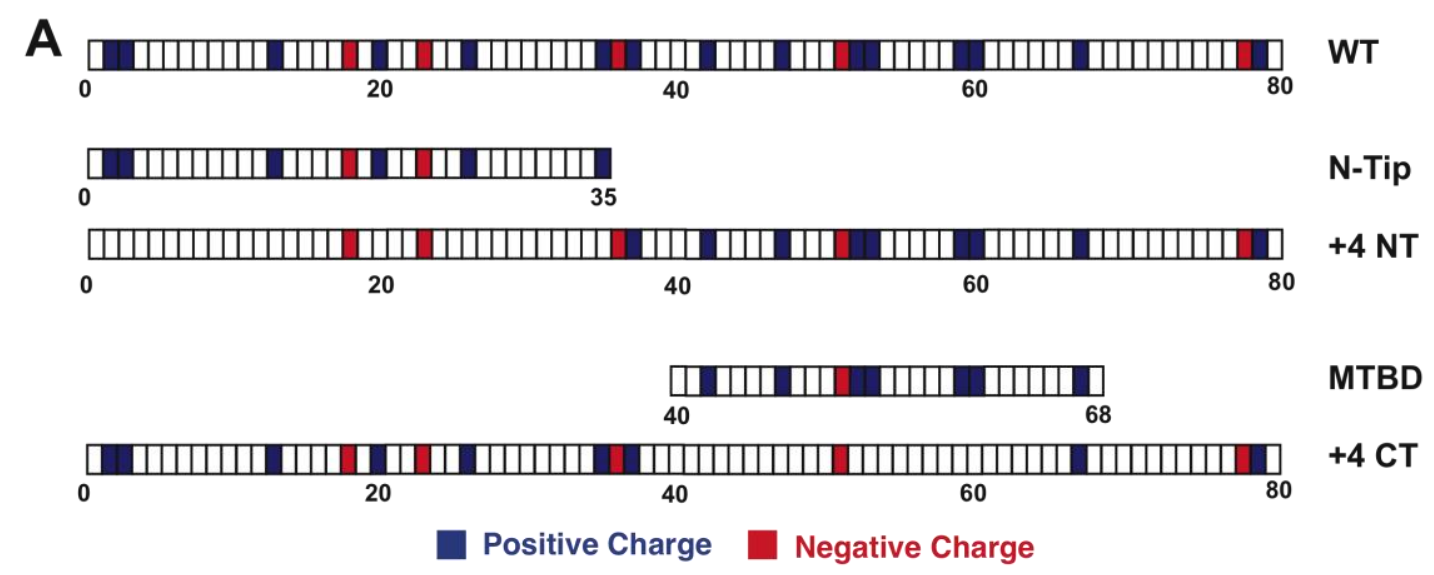

B
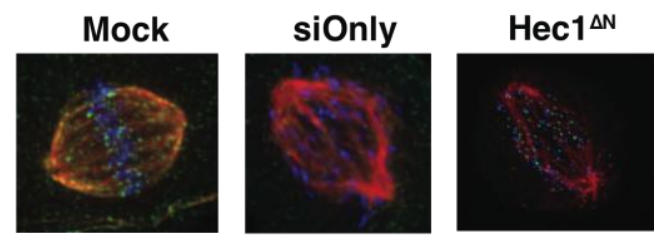

$\mathrm{Hec}^{+0}$

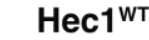

$\mathrm{Hec}^{+4 \mathrm{NT}}$

$\mathrm{Hec}^{+4 \mathrm{CT}}$

DNA
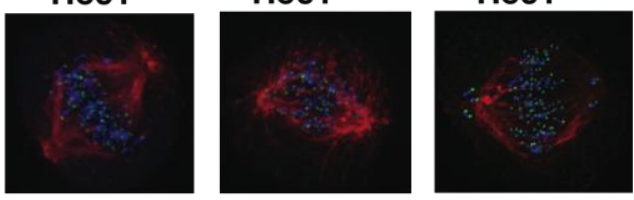

Hec1/GFP

Tubulin

C

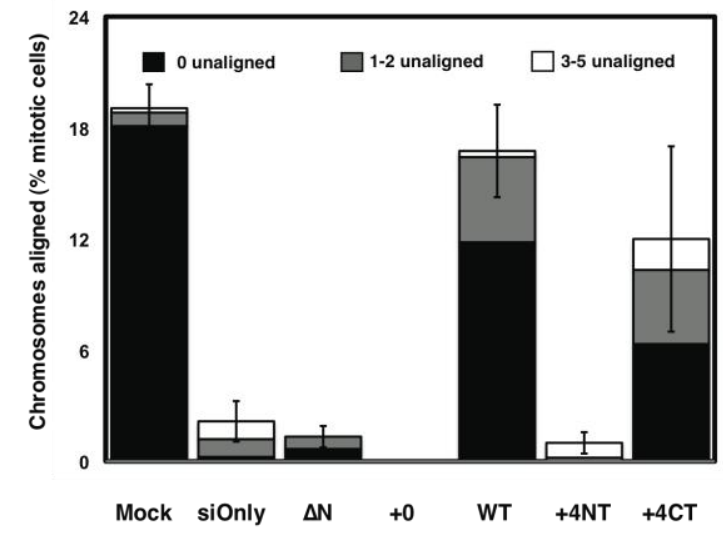

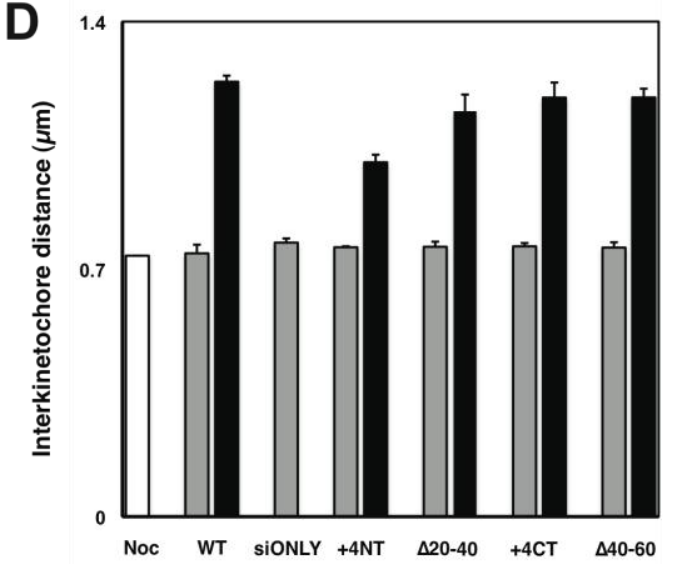

Figure 5-5 
Figure 5-6: Hec1 tail mutants arrest in metaphase in a SAC-dependant manner

(A and B) The percentage of mitotic cells in anaphase was plotted for mock treated, Hec1 knockdown, Hec $1^{\text {WT }}$ and Hec1 tail mutant cells. N=3, 100 mitotic cells counted per experiment. (B) Hec1 knockdown cells rescued with $\mathrm{Hec}^{+4 \mathrm{NT}}$ and $\mathrm{Hec}^{+4 \mathrm{CT}}$ were cotransfected with siRNA targeting Mad2. Fixation time was kept the same following release from thymidine arrest. 25 cells counted in each condition. 
A

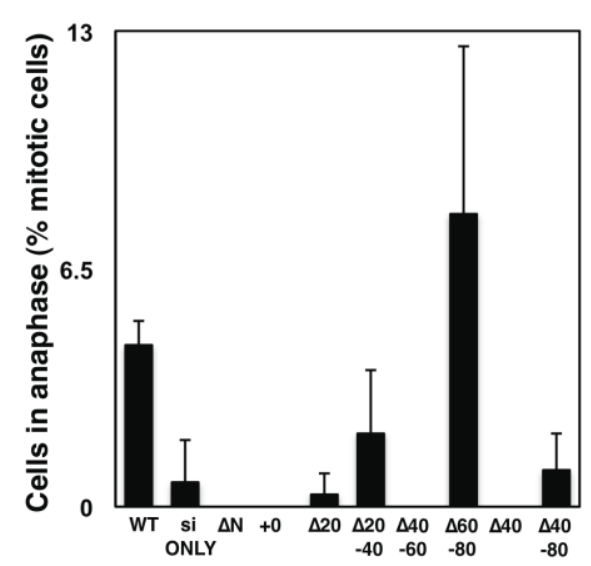

B

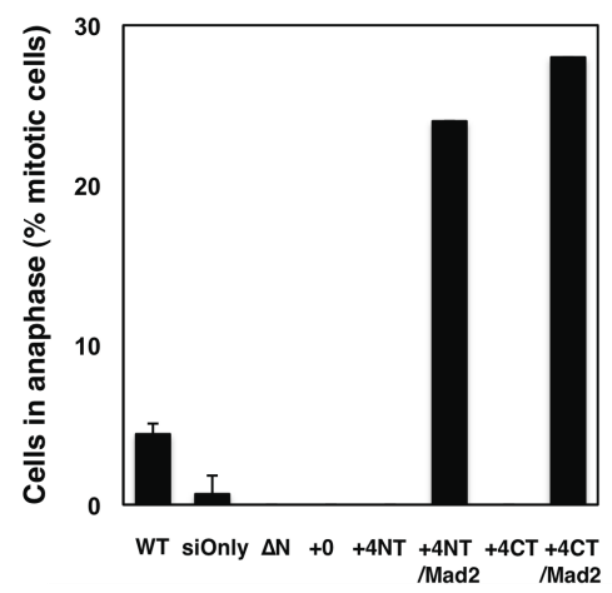

Figure 5-6 


\section{Discussion and Future Directions}

Here we extend our studies of the Ndc 80 complex by demonstrating that the $\mathrm{N}$ terminal region of the Hec1 tail, and not the MTBD, is most important for aligning chromosomes in vivo. These findings confront one of the central outstanding questions regarding vertebrate $\mathrm{Ndc} 80$ complex function - whether the $\mathrm{Hec} 1 / \mathrm{Ndc} 80$ tail is required in vivo because it provides electrostatic affinity for tubulin tails or because it generates cooperative packing of Ndc80 complexes. Our data suggest that cooperativity is the primary function of the tail, but this model requires additional testing.

\section{Affinity versus cooperativity}

The Hec1/Ndc80 tail is highly positively charged while the C-terminal tails of tubulin are highly negatively charged (Hiser et al., 2006). This simple observation has led to the hypothesis that the Hec1 tail primarily forms kinetochore-microtubule attachments through electrostatic contacts. Such an explanation fits well with Hill's theoretical model for kinetochore function, which envisions a kinetochore sleeve containing numerous lowaffinity interaction points (Hill, 1985). Additionally, a body of experimental data hints at such a mechanism for tail function. The Hec1 tail on its own binds microtubules in vitro, and binding is sensitive to both increased salt concentration and the removal of E-hooks from taxol-stabilized microtubules (Miller et al., 2008). Ndc80 complex binding to microtubules is similarly reduced following the cleavage of E-hooks from microtubules (Alushin et al., 2010; Cheeseman et al., 2006; Ciferri et al., 2008). Finally, phosphorylation of the Ndc80 complex by Aurora B kinase (which lowers the charge of the Hec1/Ndc80 tail) reduces microtubule binding affinity in vitro and compromises 
kinetochore function in vivo (Alushin et al., 2010; Cheeseman et al., 2006; Ciferri et al., 2008; DeLuca et al., 2006; Guimaraes et al., 2008; Welburn et al., 2010).

However, a model built primarily on electrostatics is not supported by other Ndc80 complex data. Most vexing is the repeated observation of cooperative binding by the Ndc80 complex to microtubules in vitro. Microtubule pelleting experiments using recombinant Ndc80 complex generate cooperative binding curves (Cheeseman et al., 2006; Tooley et al., 2011), and Ndc80 complexes can be visualized forming patches/clusters on taxol-stabilized microtubules while nearby microtubules remain undecorated (Alushin et al., 2010; Ciferri et al., 2008). Cluster formation can be disrupted not only by removing the entire tail from the Ndc80 complex but also by creating a phospho-mimetic tail mutant, inviting re-interpretations of previous phenotypes generated by Aurora B tail mutants.

Ndc80 complex function can also be altered by artificially generating complex clusters. Cross-linking Ndc80 complexes with antibodies allows Ndc80 to track a depolymerizing microtubule in conditions where single complexes cannot. Similar tiptracking is observed when Ndc80 complexes are clustered on beads (Powers et al., 2009). Additionally, injection of antibodies against the N-terminus of Hec1 leads to increased kinetochore affinity for microtubules in vivo (DeLuca et al., 2006). Therefore, an equally plausible model is that the Hec 1 tail acts as a cooperativity factor and packs Ndc80 complexes together on a microtubule to enhance processive tip tracking by the kinetochore.

The in vitro data suggest that there are two potential regions in the Hec1 tail that are capable of generating cooperative binding. In addition to possessing a high affinity 
for microtubules in vitro, a MTBD peptide is capable of forming patches on microtubules (G. Alushin, unpublished data). Cross-linking and MS/MS experiments performed on recombinant Ndc80 complex reveal that two residues from the MTBD (Lysines 53 and 59) are cross-linked to lysines in the Hec1 and Nuf2 CHDs as well as other lysines in the Hec1 tail - demonstrating that in the absence of microtubules the MTDB can contact other domains within the Ndc80 complex (Maiolica et al., 2007).

While our earlier tail truncation mutants did not reveal a second cooperative region in the Hec1/Ndc80 tail, this was likely due to the continued presence of the MTDB in our $\Delta 40$ and $\Delta 50 \mathrm{Ndc} 80^{\text {Bonsai }}$ mutants that masked the loss of the $\mathrm{N}$-Tip cooperativity region. Indeed, more recent published data provide additional evidence for the N-Tip region being involved in Ndc80 complex patch formation. First, cryo-EM tomographs of recombinant Ndc80 complex bound to a microtubule reveal regions of extra density that were not captured in the crystal structure of the Ndc80 complex, which lacked the unstructured $\mathrm{Hec} 1 / \mathrm{Ndc} 80$ tail. One region of extra density lies close to the predicted location of the N-Tip, and importantly this density contacts adjacent CHDs. Additionally, when three of the four predicted Aurora sites in the N-Tip are mutated from serine to aspartic acid to mimic phosphorylation, Ndc80 complex binding to microtubules is greatly reduced. By contrast, mutating four Aurora B sites in the MTBD generates less severe binding defects (G. Alushin, unpublished data). As the 1-40 region of the tail cannot bind microtubules on its own (G. Alushin, unpublished data), the in vitro data therefore suggest that the N-Tip is responsible for facilitating oligomerization.

The MTBD would seem to be the most likely candidate for a critical in vivo alignment factor, since it has an affinity for microtubules and can generate cooperative 
binding. However, we find just the opposite to be true. Mutants targeting this region of the tail $\left(\mathrm{Hec}^{+4 \mathrm{CT}}\right.$, Hec $1^{\Delta 40-60}$ and $\left.\mathrm{Hec}^{\Delta 40-80}\right)$ do not generate severe chromosome alignment phenotypes but fail to progress into anaphase because they are arrested by the spindle checkpoint. This suggests that while not generating severe alignment phenotypes, these mutants are nonetheless causing attachment defects that are detected by the SAC. We looked for reduced inter-kinetochore stretch in metaphase cells expressing a Hec1 MTDB mutant - one of the most likely causes of checkpoint activation - and found that wild-type levels of tension were generated on our mutant kinetochores. We also examined whether bi-oriented kinetochores in MTDB mutant cells were not parallel with the pole-to-pole spindle axis (Draviam et al., 2006) and again found no gross defect in kinetochore bi-orientation (data not shown). However, our preliminary data do indicate that cells expressing MTBD mutant proteins mis-segregate chromosomes when they are driven into anaphase by depletion of the checkpoint protein Mad2 (Figure 5-5C).

Therefore, we conclude that our MTBD mutants are generating an attachment defect that is not captured in our analysis of fixed cells (see Future Directions).

We find more severe chromosome alignment defects when we mutate the N-Tip region of the tail. $\mathrm{Hec}^{+4 \mathrm{NT}}$ cells cannot effectively align chromosomes into a metaphase plate, but they do differ from other Hec1 mutants that severely compromise chromosome alignment. Hec1 knockdown, $\mathrm{Hec}^{\Delta \mathrm{N}}$ and $\mathrm{Hec}^{+0}$ cells all fail to bi-orient chromosomes and generate tension between sister kinetochores. Cells expressing our Hec $1^{+4 \mathrm{NT}}$ mutant, in contrast, bi-orient chromosomes and generate reduced levels of tension across sister kinetochores. This indicates the C-terminal region of the tail does contribute to microtubule binding and chromosome congression (although in the presence of the N-Tip 
this contribution is not essential). Finally, we note that the $\mathrm{Hec} 1^{+4 \mathrm{NT}}$ mutant has a more severe chromosome alignment phenotype than the $\mathrm{Hec} 1^{\Delta 40}$ mutant. This may reflect an inhibitory function present in our $\mathrm{Hec} 1^{+4 \mathrm{NT}}$ mutant that is lost following removal of the entire half of the tail. Nevertheless, our data strongly suggest that the N-Tip is critical for Hec1 tail function in vivo because it brings adjacent Ndc 80 complexes close to one another on a microtubule. Such a conclusion can be significantly bolstered by additional in vitro experiments using these N-Tip mutants (see Future Directions).

\section{Regulation of the Hec1 tail}

We recently published a model for regulation of the Ndc80 complex by the Aurora B kinase (Chapter 3). In this model, we postulated that different levels of Hec1/Ndc80 tail phosphorylation could drive alternative states of Ndc80 complex interaction with microtubules. A fully phosphorylated tail would be released from a microtubule (error correction mode) while an unphosphorylated tail would allow tight packing of Ndc80 complexes along a microtubule protofilament (cooperative mode). We further speculated that the tail could also exist in a temporary state of intermediate phosphorylation (tail tethering mode), whereby low levels of phosphorylation would loosen but not dislodge the $\mathrm{Ndc} 80$ complex from a depolymerizing microtubule. This state would allow for the biased diffusion of the Ndc80 complex along a microtubule.

At the time our model was published, it was unknown whether individual Aurora B sites made differential contributions to $\mathrm{Hec} 1 / \mathrm{Ndc} 80$ tail function. However, a recent study by the DeLuca laboratory has begun to shed light on the temporal patterns on Hec1 tail phosphorylation as cells traverse mitosis (DeLuca et al., 2011). Three findings from 
their study are particularly intriguing in light of our recent findings regarding the N-Tip and MTBD. First, a key prediction of our tail regulation model is that all tail phosphorylation should be eliminated by the time the Ndc80 would need to tightly pack on a microtubule - namely in anaphase. However, the authors of this study use phosphospecific antibodies to demonstrate that phosphorylation of numerous Aurora B sites not only occurs in anaphase (albeit at reduced levels from prometaphase) but also is increased from the low levels seen in metaphase. This increase was most pronounced for Aurora B sites located in the N-Tip region (Serines 8 and 15). Second, the authors show a clear correlation between temporal phosphorylation patterns for these two Aurora B sites in the N-Tip and also for two residues in the MTBD (Serines 44 and 55). The patterns of the two pairs differ, however, suggesting differential regulation of the N-Tip and MTDB by Aurora B. Finally, the authors make an interesting observation. When mitotic chromosomes are first allowed to form bi-polar attachments to microtubules and progress into metaphase, and then are treated with nocodazole to depolymerize spindle microtubules, the authors find that Hec1 phosphorylation levels do not revert to the high levels seen in cells that had not yet bound spindle microtubules. This suggests that once the Hec1 tail contacts a microtubule a change occurs which now prevents the tail from being fully re-phosphorylated in response to a lack of microtubule tension. The authors speculate that this phenomenon could be caused by recruitment of a protein to the kinetochore that blocks tail phosphorylation. However, an alternative interpretation of this experiment is that following microtubule attachment regions of the tail containing these sites now are buried and become inaccessible to Aurora B phosphorylation - a situation that conceivably could arise in a cooperative binding arrangement. As serine 55 
is the site most refractory to re-phosphorylation in this experiment (less than $10 \%$ recovery to phosphorylation levels seen in early mitosis), it is tempting to speculate that the MTBD undergoes a conformational change following microtubule attachment. The reason for such a switch to occur remains uncertain. Nevertheless, the combination of our data and the data from the DeLuca laboratory strongly suggest that previous models for Aurora B tail regulation may be overly simplistic and in need of revision.

\section{Cooperativity factors in vertebrates, yeast}

For more than 25 years researchers have sought to understand how coupling is achieved at the kinetochore-microtubule interface. The Ndc80 complex is critical player required for depolymerization-coupled movement in all species tested, but a unifying mechanism for Ndc80-based coupling has remained elusive. In light of our data, we propose that in vertebrates the $\mathrm{Hec} 1 / \mathrm{Ndc} 80$ tail has evolved to serve as a cooperativity factor that increases the processivity of a kinetochore bound to a depolymerizing microtubule. In budding yeast, where the $\mathrm{Hec} 1 / \mathrm{Ndc} 80$ tail is dispensible, we propose that the Dam1 complex serves as a cooperativity factor that enhances Ndc80 binding to microtubules.

Several lines of evidence support this model. First, in vitro microtubule binding data involving the Ndc80 complex can increasingly be explained by loss of cooperative packing as opposed to loss of electrostatic affinity. It is important to remember that in vitro the budding yeast $\mathrm{Ndc} 80$ complex binds microtubules in a non-cooperative fashion but shows severely reduced binding following loss of its N-terminal tail (Wei et al., 2007). This experimental setup lacked the Dam1 complex (the cooperativity factor) but 
contained a highly basic unstructured tail (net charge of +9 in budding yeast). Therefore, budding yeast $\mathrm{Ndc} 80$ complex, while not requiring a $\mathrm{N}$-terminal tail for in vivo function, still relies on the tail for binding affinity in vitro. Interestingly, oligomerizing both budding yeast and human Ndc80 complexes on beads allows them to track microtubules in a similar manner (Powers et al., 2009), further arguing that artificially inducing oligomerization enhances the microtubule tracking ability for both species.

In vivo, the Dam1 complex localizes to an ideal region to act as a coupler for the Ndc80 complex. Immunofluorescence measurements made in budding yeast have localized Dam1 10 nm closer to the plus end of a microtubule than the N-terminal head of the Ndc80 complex, placing Dam1 complexes in a position to surround and cluster Ndc80 complexes on the microtubule lattice (Joglekar et al., 2009). Aurora B/Ipl1mediated error correction also targets different phosphorylation sites on human and budding yeast kinetochores. In humans, phosphorylation of the Hec1 tail severely compromises microtubule attachment but the same phenotype is not seen in budding yeast (Akiyoshi et al., 2009; DeLuca et al., 2006). Instead, a key target of Ipl1 (Aurora B) in budding yeast is a site on Dam1 that disrupts that interaction between the Ndc80 and Dam1 complexes (Lampert et al.; Shang et al., 2003). Therefore, it is tempting to speculate that a key target of the Aurora B-mediated error correction process is the site responsible for generating a processive microtubule binding site, though the targets differ across species.

As both the human and budding yeast Ndc80 tails are highly basic and contain numerous Aurora B target residues, it remains uncertain what exactly about the human Ndc80 complex facilitates cooperative binding. One idea is that residues in the human 
Hec1 or Nuf2 CHDs form interactions with the unstructured tail, but these CHD residues are not conserved in budding yeast. In support of this model, sequence comparisons between the two species reveal a CHD region conserved in metazoans (but not in yeast) that corresponds to an area of contact seen between adjacent $\mathrm{Ndc} 80^{\text {Bonsai }}$ complexes bound to a microtubule (Alushin et al., 2010). Microtubule binding of the vertebrate Ndc80 complex could be further enhanced by other kinetochore-associated factors not present in budding yeast. The Ska complex currently stands as the best candidate to functionally complement the Dam1 complex in vertebrates, but this argument remains controversial (see Chapter 3), (Daum et al., 2011; Daum et al., 2009). Future studies will be required to better test this model.

\section{Future directions}

Our data demonstrate that the N-terminal half of the Hec1 tail is required for chromosome alignment, and based on in vitro data we surmise that cooperative packing of $\mathrm{Ndc} 80$ complexes is required for in vivo kinetochore function. However, there are additional in vitro experiments that can be performed to more rigorously test this model. Perhaps the most obvious question to ask is whether our $\mathrm{Hec} 1^{+4 \mathrm{NT}}$ mutant can cosediment with microtubules. In such a binding reaction the Ndc80 complex would be predicted to bind microtubules at a reduced level, since the MTBD and dual CHD are still present. However, one would expect cooperative binding to be lost (or severely reduced). Similarly, it would be informative to test if the $\mathrm{Hec} 1^{+4 \mathrm{NT}}$ mutant can form patches on microtubules. It would also be helpful to distinguish if our $\mathrm{Hec} 1^{+4 \mathrm{NT}}$ mutant causes a severe alignment phenotype because we have lost positive charge in this region or 
because we have destroyed consensus Aurora B sites in this region. Creating a Hec1 tail mutant where serines 4, 5 and 15 are mutated to alanine (these are the sites with altered consensus sequences) and testing this mutant in HeLa cells would help to address this question.

A limitation of our fixed-cell studies is the inability to follow the fate of Hec1 tail mutants that can align chromosomes but fail to progress into anaphase at the time of fixation. It is possible that given time these cells would eventually align all chromosomes and proceed into anaphase. Additionally, if these cells did proceed into anaphase it would be informative to know if there was an increase in chromosome segregation errors. Cells expressing Nuf2 CHD mutants align chromosomes into a transient metaphase plate but chromosomes eventually leave the plate (Sundin et al., 2011), and chromosomes have also been shown to scatter from metaphase plates when cells are extensively delayed in metaphase (Daum et al., 2011; Daum et al., 2009). Therefore, there is precedent in the literature for additional phenotypes to arise following the congression of chromosomes into a metaphase plate. Filming cells as they progress through mitosis might allow us to assign later phenotypes to Hec1 tail mutants that do not exhibit strong alignment phenotypes.

Finally, one of the most technically difficult questions to answer remains how Hec1 tail interaction partners can change based on (1) the presence/absence of microtubules, (2) the presence/absence of the remainder of the Ndc80 complex and (3) the phosphorylation state of the tail. As the Hec1/Ndc80 tail has been shown to interact with tubulin tails, with other regions of the Ndc80 complex and even with other Hec1/Ndc80 tails, teasing apart the timeline and regulation of these interactions will 
remain an obstacle to fully understanding the mechanism of kinetochore coupling to microtubules. 


\section{Acknowledgements}

We thank Eva Nogales and Greg Alushin for sharing unpublished data and for many

helpful discussions. 
References

Akiyoshi, B., Nelson, C.R., Ranish, J.A., and Biggins, S. (2009). Analysis of Ipl1mediated phosphorylation of the Ndc80 kinetochore protein in Saccharomyces cerevisiae. Genetics 183, 1591-1595.

Al-Bassam, J., Kim, H., Brouhard, G., van Oijen, A., Harrison, S.C., and Chang, F. (2010). CLASP promotes microtubule rescue by recruiting tubulin dimers to the microtubule. Dev Cell 19, 245-258.

Alushin, G.M., Ramey, V.H., Pasqualato, S., Ball, D.A., Grigorieff, N., Musacchio, A., and Nogales, E. (2010). The Ndc80 kinetochore complex forms oligomeric arrays along microtubules. Nature 467, 805-810.

Andersen, J.S., Wilkinson, C.J., Mayor, T., Mortensen, P., Nigg, E.A., and Mann, M. (2003). Proteomic characterization of the human centrosome by protein correlation profiling. Nature 426, 570-574.

Andersen, S.S., and Wittmann, T. (2002). Toward reconstitution of in vivo microtubule dynamics in vitro. Bioessays 24, 305-307.

Asbury, C.L., Gestaut, D.R., Powers, A.F., Franck, A.D., and Davis, T.N. (2006). The Dam1 kinetochore complex harnesses microtubule dynamics to produce force and movement. Proc Natl Acad Sci U S A 103, 9873-9878.

Asbury, C.L., Tien, J.F., and Davis, T.N. (2011). Kinetochores' gripping feat: conformational wave or biased diffusion? Trends Cell Biol 21, 38-46.

Asteriti, I.A., Rensen, W.M., Lindon, C., Lavia, P., and Guarguaglini, G. (2010). The Aurora-A/TPX2 complex: a novel oncogenic holoenzyme? Biochim Biophys Acta 1806, 230-239.

Barisic, M., Sohm, B., Mikolcevic, P., Wandke, C., Rauch, V., Ringer, T., Hess, M., Bonn, G., and Geley, S. (2010). Spindly/CCDC99 is required for efficient chromosome congression and mitotic checkpoint regulation. Mol Biol Cell 21, 1968-1981.

Bharadwaj, R., Qi, W., and Yu, H. (2004). Identification of two novel components of the human NDC80 kinetochore complex. J Biol Chem 279, 13076-13085.

Biggins, S., and Murray, A.W. (2001). The budding yeast protein kinase Ipl1/Aurora allows the absence of tension to activate the spindle checkpoint. Genes Dev 15, 31183129.

Bloom, K. (2008). Kinetochores and microtubules wed without a ring. Cell 135, 211-213. 
Bossard,C., Laurell, H., Van den Berghe, L., Meunier, S., Zanibellato, C., and Prats, H. (2003). Translokin is an intracellular mediator of FGF-2 trafficking. Nat Cell Biol 5, 433439.

Breuer, M., Kolano, A., Kwon, M., Li, C.C., Tsai, T.F., Pellman, D., Brunet, S., and Verlhac, M.H. (2010). HURP permits MTOC sorting for robust meiotic spindle bipolarity, similar to extra centrosome clustering in cancer cells. J Cell Biol 191, 12511260.

Brinkley, B.R., and Cartwright, J., Jr. (1975). Cold-labile and cold-stable microtubules in the mitotic spindle of mammalian cells. Ann N Y Acad Sci 253, 428-439.

Brinkley, B.R., and Stubblefield, E. (1966). The fine structure of the kinetochore of a mammalian cell in vitro. Chromosoma 19, 28-43.

Brouhard, G.J., Stear, J.H., Noetzel, T.L., Al-Bassam, J., Kinoshita, K., Harrison, S.C., Howard, J., and Hyman, A.A. (2008). XMAP215 is a processive microtubule polymerase. Cell 132, 79-88.

Burke, D.J., and Stukenberg, P.T. (2008). Linking kinetochore-microtubule binding to the spindle checkpoint. Dev Cell 14, 474-479.

Carazo-Salas, R.E., Guarguaglini, G., Gruss, O.J., Segref, A., Karsenti, E., and Mattaj, I.W. (1999). Generation of GTP-bound Ran by RCC1 is required for chromatin-induced mitotic spindle formation. Nature 400, 178-181.

Carmena, M., and Earnshaw, W.C. (2003). The cellular geography of aurora kinases. Nat Rev Mol Cell Biol 4, 842-854.

Carroll, C.W., Milks, K.J., and Straight, A.F. (2010). Dual recognition of CENP-A nucleosomes is required for centromere assembly. J Cell Biol 189, 1143-1155.

Casanova, C.M., Rybina, S., Yokoyama, H., Karsenti, E., and Mattaj, I.W. (2008). Hepatoma up-regulated protein is required for chromatin-induced microtubule assembly independently of TPX2. Mol Biol Cell 19, 4900-4908.

Chan, G.K., Jablonski, S.A., Starr, D.A., Goldberg, M.L., and Yen, T.J. (2000). Human Zw10 and ROD are mitotic checkpoint proteins that bind to kinetochores. Nat Cell Biol 2, 944-947.

Cheeseman, I.M., Anderson, S., Jwa, M., Green, E.M., Kang, J., Yates, J.R., 3rd, Chan, C.S., Drubin, D.G., and Barnes, G. (2002). Phospho-regulation of kinetochoremicrotubule attachments by the Aurora kinase Ipl1p. Cell 111, 163-172. 
Cheeseman, I.M., Chappie, J.S., Wilson-Kubalek, E.M., and Desai, A. (2006). The conserved KMN network constitutes the core microtubule-binding site of the kinetochore. Cell 127, 983-997.

Cheeseman, I.M., and Desai, A. (2008). Molecular architecture of the kinetochoremicrotubule interface. Nat Rev Mol Cell Biol 9, 33-46.

Cheeseman, I.M., Niessen, S., Anderson, S., Hyndman, F., Yates, J.R., 3rd, Oegema, K., and Desai, A. (2004). A conserved protein network controls assembly of the outer kinetochore and its ability to sustain tension. Genes Dev 18, 2255-2268.

Chen, Y., Riley, D.J., Chen, P.L., and Lee, W.H. (1997). HEC, a novel nuclear protein rich in leucine heptad repeats specifically involved in mitosis. Mol Cell Biol 17, 60496056.

Chen, Y., Riley, D.J., Zheng, L., Chen, P.L., and Lee, W.H. (2002). Phosphorylation of the mitotic regulator protein Hec1 by Nek2 kinase is essential for faithful chromosome segregation. J Biol Chem 277, 49408-49416.

Ciferri, C., De Luca, J., Monzani, S., Ferrari, K.J., Ristic, D., Wyman, C., Stark, H., Kilmartin, J., Salmon, E.D., and Musacchio, A. (2005). Architecture of the human ndc80hec1 complex, a critical constituent of the outer kinetochore. J Biol Chem 280, 2908829095.

Ciferri, C., Musacchio, A., and Petrovic, A. (2007). The Ndc80 complex: hub of kinetochore activity. FEBS Lett 581, 2862-2869.

Ciferri, C., Pasqualato, S., Screpanti, E., Varetti, G., Santaguida, S., Dos Reis, G., Maiolica, A., Polka, J., De Luca, J.G., De Wulf, P., et al. (2008). Implications for kinetochore-microtubule attachment from the structure of an engineered Ndc80 complex. Cell 133, 427-439.

Cimini, D., Howell, B., Maddox, P., Khodjakov, A., Degrassi, F., and Salmon, E.D. (2001). Merotelic kinetochore orientation is a major mechanism of aneuploidy in mitotic mammalian tissue cells. J Cell Biol 153, 517-527.

Cimini, D., Moree, B., Canman, J.C., and Salmon, E.D. (2003). Merotelic kinetochore orientation occurs frequently during early mitosis in mammalian tissue cells and error correction is achieved by two different mechanisms. J Cell Sci 116, 4213-4225.

Cleveland, D.W., Mao, Y., and Sullivan, K.F. (2003). Centromeres and kinetochores: from epigenetics to mitotic checkpoint signaling. Cell 112, 407-421.

Dai, J., Sultan, S., Taylor, S.S., and Higgins, J.M. (2005). The kinase haspin is required for mitotic histone $\mathrm{H} 3 \mathrm{Thr} 3$ phosphorylation and normal metaphase chromosome alignment. Genes Dev 19, 472-488. 
Daum, J.R., Potapova, T.A., Sivakumar, S., Daniel, J.J., Flynn, J.N., Rankin, S., and Gorbsky, G.J. (2011). Cohesion fatigue induces chromatid separation in cells delayed at metaphase. Curr Biol 21, 1018-1024.

Daum, J.R., Wren, J.D., Daniel, J.J., Sivakumar, S., McAvoy, J.N., Potapova, T.A., and Gorbsky, G.J. (2009). Ska3 is required for spindle checkpoint silencing and the maintenance of chromosome cohesion in mitosis. Curr Biol 19, 1467-1472.

Davis, T.N., and Wordeman, L. (2007). Rings, bracelets, sleeves, and chevrons: new structures of kinetochore proteins. Trends Cell Biol 17, 377-382.

DeLuca, J.G., Dong, Y., Hergert, P., Strauss, J., Hickey, J.M., Salmon, E.D., and McEwen, B.F. (2005). Hec1 and nuf2 are core components of the kinetochore outer plate essential for organizing microtubule attachment sites. Mol Biol Cell 16, 519-531.

DeLuca, J.G., Gall, W.E., Ciferri, C., Cimini, D., Musacchio, A., and Salmon, E.D. (2006). Kinetochore microtubule dynamics and attachment stability are regulated by Hec1. Cell 127, 969-982.

DeLuca, J.G., Howell, B.J., Canman, J.C., Hickey, J.M., Fang, G., and Salmon, E.D. (2003). Nuf2 and Hec1 are required for retention of the checkpoint proteins Mad1 and Mad2 to kinetochores. Curr Biol 13, 2103-2109.

DeLuca, J.G., Moree, B., Hickey, J.M., Kilmartin, J.V., and Salmon, E.D. (2002). hNuf2 inhibition blocks stable kinetochore-microtubule attachment and induces mitotic cell death in HeLa cells. J Cell Biol 159, 549-555.

DeLuca, K.F., Lens, S.M., and DeLuca, J.G. (2011). Temporal changes in Hec1 phosphorylation control kinetochore-microtubule attachment stability during mitosis. J Cell Sci 124, 622-634.

Derewenda, U., Tarricone, C., Choi, W.C., Cooper, D.R., Lukasik, S., Perrina, F., Tripathy, A., Kim, M.H., Cafiso, D.S., Musacchio, A., et al. (2007). The structure of the coiled-coil domain of Ndel1 and the basis of its interaction with Lis1, the causal protein of Miller-Dieker lissencephaly. Structure 15, 1467-1481.

Desai, A., Murray, A., Mitchison, T.J., and Walczak, C.E. (1999a). The use of Xenopus egg extracts to study mitotic spindle assembly and function in vitro. Methods Cell Biol $61,385-412$.

Desai, A., Rybina, S., Muller-Reichert, T., Shevchenko, A., Hyman, A., and Oegema, K. (2003). KNL-1 directs assembly of the microtubule-binding interface of the kinetochore in C. elegans. Genes Dev 17, 2421-2435. 
Desai, A., Verma, S., Mitchison, T.J., and Walczak, C.E. (1999b). Kin I kinesins are microtubule-destabilizing enzymes. Cell 96, 69-78.

Diaz-Rodriguez, E., Sotillo, R., Schvartzman, J.M., and Benezra, R. (2008). Hec1 overexpression hyperactivates the mitotic checkpoint and induces tumor formation in vivo. Proc Natl Acad Sci U S A 105, 16719-16724.

Ding, R., McDonald, K.L., and McIntosh, J.R. (1993). Three-dimensional reconstruction and analysis of mitotic spindles from the yeast, Schizosaccharomyces pombe. J Cell Biol $120,141-151$.

Dong, Y., Vanden Beldt, K.J., Meng, X., Khodjakov, A., and McEwen, B.F. (2007). The outer plate in vertebrate kinetochores is a flexible network with multiple microtubule interactions. Nat Cell Biol 9, 516-522.

Draviam, V.M., Shapiro, I., Aldridge, B., and Sorger, P.K. (2006). Misorientation and reduced stretching of aligned sister kinetochores promote chromosome missegregation in EB1- or APC-depleted cells. EMBO J 25, 2814-2827.

Du, J., Cai, X., Yao, J., Ding, X., Wu, Q., Pei, S., Jiang, K., Zhang, Y., Wang, W., Shi, Y., et al. (2008). The mitotic checkpoint kinase NEK2A regulates kinetochore microtubule attachment stability. Oncogene 27, 4107-4114.

Dumont, J., Petri, S., Pellegrin, F., Terret, M.E., Bohnsack, M.T., Rassinier, P., Georget, V., Kalab, P., Gruss, O.J., and Verlhac, M.H. (2007). A centriole- and RanGTPindependent spindle assembly pathway in meiosis I of vertebrate oocytes. J Cell Biol 176, 295-305.

Earnshaw, W.C., and Rothfield, N. (1985). Identification of a family of human centromere proteins using autoimmune sera from patients with scleroderma. Chromosoma 91, 313-321.

Emanuele, M.J., McCleland, M.L., Satinover, D.L., and Stukenberg, P.T. (2005). Measuring the stoichiometry and physical interactions between components elucidates the architecture of the vertebrate kinetochore. Mol Biol Cell 16, 4882-4892.

Emanuele, M.J., and Stukenberg, P.T. (2007). Xenopus Cep57 is a novel kinetochore component involved in microtubule attachment. Cell 130, 893-905.

Famulski, J.K., Vos, L.J., Rattner, J.B., and Chan, G.K. (2011). Dynein/Dynactinmediated transport of kinetochore components off kinetochores and onto spindle poles induced by nordihydroguaiaretic acid. PLoS One 6, e16494.

Feng, J., Huang, H., and Yen, T.J. (2006). CENP-F is a novel microtubule-binding protein that is essential for kinetochore attachments and affects the duration of the mitotic checkpoint delay. Chromosoma 115, 320-329. 
Foltz, D.R., Jansen, L.E., Black, B.E., Bailey, A.O., Yates, J.R., 3rd, and Cleveland, D.W. (2006). The human CENP-A centromeric nucleosome-associated complex. Nat Cell Biol 8, 458-469.

Franco, A., Meadows, J.C., and Millar, J.B. (2007). The Dam1/DASH complex is required for the retrieval of unclustered kinetochores in fission yeast. J Cell Sci 120, 3345-3351.

Gao, Q., Courtheoux, T., Gachet, Y., Tournier, S., and He, X. (2010). A non-ring-like form of the Dam1 complex modulates microtubule dynamics in fission yeast. Proc Natl Acad Sci U S A 107, 13330-13335.

Gascoigne, K.E., and Cheeseman, I.M. (2011). Kinetochore assembly: if you build it, they will come. Curr Opin Cell Biol 23, 102-108.

Gascoigne, K.E., Takeuchi, K., Suzuki, A., Hori, T., Fukagawa, T., and Cheeseman, I.M. (2011). Induced ectopic kinetochore assembly bypasses the requirement for CENP-A nucleosomes. Cell 145, 410-422.

Gassmann, R., Essex, A., Hu, J.S., Maddox, P.S., Motegi, F., Sugimoto, A., O'Rourke, S.M., Bowerman, B., McLeod, I., Yates, J.R., 3rd, et al. (2008). A new mechanism controlling kinetochore-microtubule interactions revealed by comparison of two dyneintargeting components: SPDL-1 and the Rod/Zwilch/Zw10 complex. Genes Dev 22, 23852399.

Gassmann, R., Holland, A.J., Varma, D., Wan, X., Civril, F., Cleveland, D.W., Oegema, K., Salmon, E.D., and Desai, A. (2010). Removal of Spindly from microtubule-attached kinetochores controls spindle checkpoint silencing in human cells. Genes Dev 24, 957971.

Gestaut, D.R., Graczyk, B., Cooper, J., Widlund, P.O., Zelter, A., Wordeman, L., Asbury, C.L., and Davis, T.N. (2008). Phosphoregulation and depolymerization-driven movement of the Dam1 complex do not require ring formation. Nat Cell Biol 10, 407-414.

Gordon, M.B., Howard, L., and Compton, D.A. (2001). Chromosome movement in mitosis requires microtubule anchorage at spindle poles. J Cell Biol 152, 425-434.

Goto, H., Kiyono, T., Tomono, Y., Kawajiri, A., Urano, T., Furukawa, K., Nigg, E.A., and Inagaki, M. (2006). Complex formation of Plk1 and INCENP required for metaphase-anaphase transition. Nat Cell Biol 8, 180-187.

Grabsch, H., Takeno, S., Parsons, W.J., Pomjanski, N., Boecking, A., Gabbert, H.E., and Mueller, W. (2003). Overexpression of the mitotic checkpoint genes BUB1, BUBR1, and BUB3 in gastric cancer--association with tumour cell proliferation. J Pathol 200, 16-22. 
Grishchuk, E.L., Efremov, A.K., Volkov, V.A., Spiridonov, I.S., Gudimchuk, N.,

Westermann, S., Drubin, D., Barnes, G., McIntosh, J.R., and Ataullakhanov, F.I. (2008a).

The Dam1 ring binds microtubules strongly enough to be a processive as well as energyefficient coupler for chromosome motion. Proc Natl Acad Sci U S A 105, 15423-15428.

Grishchuk, E.L., Spiridonov, I.S., Volkov, V.A., Efremov, A., Westermann, S., Drubin, D., Barnes, G., Ataullakhanov, F.I., and McIntosh, J.R. (2008b). Different assemblies of the DAM1 complex follow shortening microtubules by distinct mechanisms. Proc Natl Acad Sci U S A 105, 6918-6923.

Gruss, O.J., Carazo-Salas, R.E., Schatz, C.A., Guarguaglini, G., Kast, J., Wilm, M., Le Bot, N., Vernos, I., Karsenti, E., and Mattaj, I.W. (2001). Ran induces spindle assembly by reversing the inhibitory effect of importin alpha on TPX2 activity. Cell 104, 83-93.

Guimaraes, G.J., Dong, Y., McEwen, B.F., and Deluca, J.G. (2008). Kinetochoremicrotubule attachment relies on the disordered N-terminal tail domain of Hec1. Curr Biol 18, 1778-1784.

Hanisch, A., Sillje, H.H., and Nigg, E.A. (2006). Timely anaphase onset requires a novel spindle and kinetochore complex comprising Ska1 and Ska2. EMBO J 25, 5504-5515.

Hauf, S., Cole, R.W., LaTerra, S., Zimmer, C., Schnapp, G., Walter, R., Heckel, A., van Meel, J., Rieder, C.L., and Peters, J.M. (2003). The small molecule Hesperadin reveals a role for Aurora B in correcting kinetochore-microtubule attachment and in maintaining the spindle assembly checkpoint. J Cell Biol 161, 281-294.

Hayashi, I., and Ikura, M. (2003). Crystal structure of the amino-terminal microtubulebinding domain of end-binding protein 1 (EB1). J Biol Chem 278, 36430-36434.

Hayashi, I., Wilde, A., Mal, T.K., and Ikura, M. (2005). Structural basis for the activation of microtubule assembly by the EB1 and p150Glued complex. Mol Cell 19, 449-460.

Hill, T.L. (1985). Theoretical problems related to the attachment of microtubules to kinetochores. Proc Natl Acad Sci U S A 82, 4404-4408.

Hiser, L., Aggarwal, A., Young, R., Frankfurter, A., Spano, A., Correia, J.J., and Lobert, S. (2006). Comparison of beta-tubulin mRNA and protein levels in 12 human cancer cell lines. Cell Motil Cytoskeleton 63, 41-52.

Hoffman, D.B., Pearson, C.G., Yen, T.J., Howell, B.J., and Salmon, E.D. (2001). Microtubule-dependent changes in assembly of microtubule motor proteins and mitotic spindle checkpoint proteins at PtK1 kinetochores. Mol Biol Cell 12, 1995-2009.

Holland, A.J., and Cleveland, D.W. (2009). Boveri revisited: chromosomal instability, aneuploidy and tumorigenesis. Nat Rev Mol Cell Biol 10, 478-487. 
Hori, T., Amano, M., Suzuki, A., Backer, C.B., Welburn, J.P., Dong, Y., McEwen, B.F., Shang, W.H., Suzuki, E., Okawa, K., et al. (2008). CCAN makes multiple contacts with centromeric DNA to provide distinct pathways to the outer kinetochore. Cell 135, 10391052.

Hori, T., Haraguchi, T., Hiraoka, Y., Kimura, H., and Fukagawa, T. (2003). Dynamic behavior of Nuf2-Hec1 complex that localizes to the centrosome and centromere and is essential for mitotic progression in vertebrate cells. J Cell Sci 116, 3347-3362.

Howard, J., and Hyman, A.A. (2003). Dynamics and mechanics of the microtubule plus end. Nature 422, 753-758.

Howell, B.J., McEwen, B.F., Canman, J.C., Hoffman, D.B., Farrar, E.M., Rieder, C.L., and Salmon, E.D. (2001). Cytoplasmic dynein/dynactin drives kinetochore protein transport to the spindle poles and has a role in mitotic spindle checkpoint inactivation. $\mathrm{J}$ Cell Biol 155, 1159-1172.

Howman, E.V., Fowler, K.J., Newson, A.J., Redward, S., MacDonald, A.C., Kalitsis, P., and Choo, K.H. (2000). Early disruption of centromeric chromatin organization in centromere protein A (Cenpa) null mice. Proc Natl Acad Sci U S A 97, 1148-1153.

Hoyt, M.A., Totis, L., and Roberts, B.T. (1991). S. cerevisiae genes required for cell cycle arrest in response to loss of microtubule function. Cell 66, 507-517.

Hsu, K.S., and Toda, T. Ndc80 internal loop interacts with Dis1/TOG to ensure proper kinetochore-spindle attachment in fission yeast. Curr Biol 21, 214-220.

Huang, H., Feng, J., Famulski, J., Rattner, J.B., Liu, S.T., Kao, G.D., Muschel, R., Chan, G.K., and Yen, T.J. (2007). Tripin/hSgo2 recruits MCAK to the inner centromere to correct defective kinetochore attachments. J Cell Biol 177, 413-424.

Hunt, A.J., and McIntosh, J.R. (1998). The dynamic behavior of individual microtubules associated with chromosomes in vitro. Mol Biol Cell 9, 2857-2871.

Izuta, H., Ikeno, M., Suzuki, N., Tomonaga, T., Nozaki, N., Obuse, C., Kisu, Y., Goshima, N., Nomura, F., Nomura, N., et al. (2006). Comprehensive analysis of the ICEN (Interphase Centromere Complex) components enriched in the CENP-A chromatin of human cells. Genes Cells 11, 673-684.

Janke, C., Ortiz, J., Lechner, J., Shevchenko, A., Magiera, M.M., Schramm, C., and Schiebel, E. (2001). The budding yeast proteins Spc24p and Spc25p interact with Ndc80p and Nuf $2 \mathrm{p}$ at the kinetochore and are important for kinetochore clustering and checkpoint control. EMBO J 20, 777-791.

Joglekar, A.P., Bloom, K., and Salmon, E.D. (2009). In vivo protein architecture of the eukaryotic kinetochore with nanometer scale accuracy. Curr Biol 19, 694-699. 
Joglekar, A.P., Bloom, K.S., and Salmon, E.D. Mechanisms of force generation by endon kinetochore-microtubule attachments. Curr Opin Cell Biol 22, 57-67.

Joglekar, A.P., Bouck, D., Finley, K., Liu, X., Wan, Y., Berman, J., He, X., Salmon, E.D., and Bloom, K.S. (2008). Molecular architecture of the kinetochore-microtubule attachment site is conserved between point and regional centromeres. J Cell Biol 181, 587-594.

Joglekar, A.P., Bouck, D.C., Molk, J.N., Bloom, K.S., and Salmon, E.D. (2006).

Molecular architecture of a kinetochore-microtubule attachment site. Nat Cell Biol 8, 581-585.

Johnston, K., Joglekar, A., Hori, T., Suzuki, A., Fukagawa, T., and Salmon, E.D. Vertebrate kinetochore protein architecture: protein copy number. J Cell Biol 189, 937943.

Joseph, J., Liu, S.T., Jablonski, S.A., Yen, T.J., and Dasso, M. (2004). The RanGAP1RanBP2 complex is essential for microtubule-kinetochore interactions in vivo. Curr Biol 14, 611-617.

Kalab, P., Pralle, A., Isacoff, E.Y., Heald, R., and Weis, K. (2006). Analysis of a RanGTP-regulated gradient in mitotic somatic cells. Nature 440, 697-701.

Kapoor, T.M., Lampson, M.A., Hergert, P., Cameron, L., Cimini, D., Salmon, E.D., McEwen, B.F., and Khodjakov, A. (2006). Chromosomes can congress to the metaphase plate before biorientation. Science 311, 388-391.

Karess, R. (2005). Rod-Zw10-Zwilch: a key player in the spindle checkpoint. Trends Cell Biol 15, 386-392.

Kemmler, S., Stach, M., Knapp, M., Ortiz, J., Pfannstiel, J., Ruppert, T., and Lechner, J. (2009). Mimicking Ndc80 phosphorylation triggers spindle assembly checkpoint signalling. EMBO J 28, 1099-1110.

Khodjakov, A., and Pines, J. Centromere tension: a divisive issue. Nat Cell Biol 12, 919923.

Khodjakov, A., and Rieder, C.L. (2009). Mitosis: too much of a good thing (can be bad). Curr Biol 19, R1032-1034.

Kim, Y.S., Nakanishi, G., Oudes, A.J., Kim, K.H., Wang, H., Kilpatrick, D.L., and Jetten, A.M. (2004). Tsp57: a novel gene induced during a specific stage of spermatogenesis. Biol Reprod 70, 106-113. 
Kinoshita, K., Arnal, I., Desai, A., Drechsel, D.N., and Hyman, A.A. (2001).

Reconstitution of physiological microtubule dynamics using purified components.

Science 294, 1340-1343.

Kirschner, M.W., and Mitchison, T. (1986). Microtubule dynamics. Nature 324, 621.

Kiyomitsu, T., Murakami, H., and Yanagida, M. Protein interaction domain mapping of human kinetochore protein Blinkin reveals a consensus motif for binding of spindle assembly checkpoint proteins Bub1 and BubR1. Mol Cell Biol 31, 998-1011.

Kiyomitsu, T., Obuse, C., and Yanagida, M. (2007). Human Blinkin/AF15q14 is required for chromosome alignment and the mitotic checkpoint through direct interaction with Bub1 and BubR1. Dev Cell 13, 663-676.

Kline, S.L., Cheeseman, I.M., Hori, T., Fukagawa, T., and Desai, A. (2006). The human Mis12 complex is required for kinetochore assembly and proper chromosome segregation. J Cell Biol 173, 9-17.

Kline-Smith, S.L., Sandall, S., and Desai, A. (2005). Kinetochore-spindle microtubule interactions during mitosis. Curr Opin Cell Biol 17, 35-46.

Kline-Smith, S.L., and Walczak, C.E. (2002). The microtubule-destabilizing kinesin XKCM1 regulates microtubule dynamic instability in cells. Mol Biol Cell 13, 2718-2731.

Knowlton, A.L., Vorozhko, V.V., Lan, W., Gorbsky, G.J., and Stukenberg, P.T. (2009). ICIS and Aurora B coregulate the microtubule depolymerase Kif2a. Curr Biol 19, 758763.

Koffa, M.D., Casanova, C.M., Santarella, R., Kocher, T., Wilm, M., and Mattaj, I.W. (2006). HURP is part of a Ran-dependent complex involved in spindle formation. Curr Biol 16, 743-754.

Koshland, D.E., Mitchison, T.J., and Kirschner, M.W. (1988). Polewards chromosome movement driven by microtubule depolymerization in vitro. Nature 331, 499-504.

Kristofferson, D., Mitchison, T., and Kirschner, M. (1986). Direct observation of steadystate microtubule dynamics. J Cell Biol 102, 1007-1019.

Lampert, F., Hornung, P., and Westermann, S. The Dam1 complex confers microtubule plus end-tracking activity to the Ndc80 kinetochore complex. J Cell Biol 189, 641-649.

Lan, W., Zhang, X., Kline-Smith, S.L., Rosasco, S.E., Barrett-Wilt, G.A., Shabanowitz, J., Hunt, D.F., Walczak, C.E., and Stukenberg, P.T. (2004). Aurora B phosphorylates centromeric MCAK and regulates its localization and microtubule depolymerization activity. Curr Biol 14, 273-286. 
Lange, B.M., and Gull, K. (1995). A molecular marker for centriole maturation in the mammalian cell cycle. J Cell Biol 130, 919-927.

Li, R., and Murray, A.W. (1991). Feedback control of mitosis in budding yeast. Cell 66, 519-531.

Li, X., and Nicklas, R.B. (1995). Mitotic forces control a cell-cycle checkpoint. Nature $373,630-632$.

Liu, D., Vader, G., Vromans, M.J., Lampson, M.A., and Lens, S.M. (2009). Sensing chromosome bi-orientation by spatial separation of aurora B kinase from kinetochore substrates. Science 323, 1350-1353.

Liu, D., Vleugel, M., Backer, C.B., Hori, T., Fukagawa, T., Cheeseman, I.M., and Lampson, M.A. Regulated targeting of protein phosphatase 1 to the outer kinetochore by KNL1 opposes Aurora B kinase. J Cell Biol 188, 809-820.

Liu, X., McLeod, I., Anderson, S., Yates, J.R., 3rd, and He, X. (2005). Molecular analysis of kinetochore architecture in fission yeast. EMBO J 24, 2919-2930.

Maddox, P., Straight, A., Coughlin, P., Mitchison, T.J., and Salmon, E.D. (2003). Direct observation of microtubule dynamics at kinetochores in Xenopus extract spindles: implications for spindle mechanics. J Cell Biol 162, 377-382.

Maiolica, A., Cittaro, D., Borsotti, D., Sennels, L., Ciferri, C., Tarricone, C., Musacchio, A., and Rappsilber, J. (2007). Structural analysis of multiprotein complexes by crosslinking, mass spectrometry, and database searching. Mol Cell Proteomics 6, 2200-2211. Maresca, T.J., and Salmon, E.D. Welcome to a new kind of tension: translating kinetochore mechanics into a wait-anaphase signal. J Cell Sci 123, 825-835.

Maresca, T.J., and Salmon, E.D. (2009). Intrakinetochore stretch is associated with changes in kinetochore phosphorylation and spindle assembly checkpoint activity. J Cell Biol 184, 373-381.

Martin-Lluesma, S., Stucke, V.M., and Nigg, E.A. (2002). Role of Hec1 in spindle checkpoint signaling and kinetochore recruitment of Mad1/Mad2. Science 297, 22672270 .

Martineau-Thuillier, S., Andreassen, P.R., and Margolis, R.L. (1998). Colocalization of TD-60 and INCENP throughout G2 and mitosis: evidence for their possible interaction in signalling cytokinesis. Chromosoma 107, 461-470.

Mattiuzzo, M., Vargiu, G., Totta, P., Fiore, M., Ciferri, C., Musacchio, A., and Degrassi, F. Abnormal kinetochore-generated pulling forces from expressing a N-terminally modified Hec1. PLoS One 6, e16307. 
Maure, J.F., Komoto, S., Oku, Y., Mino, A., Pasqualato, S., Natsume, K., Clayton, L.,

Musacchio, A., and Tanaka, T.U. The Ndc80 loop region facilitates formation of kinetochore attachment to the dynamic microtubule plus end. Curr Biol 21, 207-213.

McCleland, M.L., Gardner, R.D., Kallio, M.J., Daum, J.R., Gorbsky, G.J., Burke, D.J., and Stukenberg, P.T. (2003). The highly conserved Ndc80 complex is required for kinetochore assembly, chromosome congression, and spindle checkpoint activity. Genes Dev 17, 101-114.

McCleland, M.L., Kallio, M.J., Barrett-Wilt, G.A., Kestner, C.A., Shabanowitz, J., Hunt, D.F., Gorbsky, G.J., and Stukenberg, P.T. (2004). The vertebrate Ndc80 complex contains Spc24 and Spc25 homologs, which are required to establish and maintain kinetochore-microtubule attachment. Curr Biol 14, 131-137.

McEwen, B.F., Hsieh, C.E., Mattheyses, A.L., and Rieder, C.L. (1998). A new look at kinetochore structure in vertebrate somatic cells using high-pressure freezing and freeze substitution. Chromosoma 107, 366-375.

McIntosh, J.R., Grishchuk, E.L., Morphew, M.K., Efremov, A.K., Zhudenkov, K., Volkov, V.A., Cheeseman, I.M., Desai, A., Mastronarde, D.N., and Ataullakhanov, F.I. (2008). Fibrils connect microtubule tips with kinetochores: a mechanism to couple tubulin dynamics to chromosome motion. Cell 135, 322-333.

Meraldi, P., Draviam, V.M., and Sorger, P.K. (2004). Timing and checkpoints in the regulation of mitotic progression. Dev Cell 7, 45-60.

Meraldi, P., McAinsh, A.D., Rheinbay, E., and Sorger, P.K. (2006). Phylogenetic and structural analysis of centromeric DNA and kinetochore proteins. Genome Biol 7, R23. Meunier, S., Navarro, M.G., Bossard, C., Laurell, H., Touriol, C., Lacazette, E., and Prats, H. (2009). Pivotal role of translokin/CEP57 in the unconventional secretion versus nuclear translocation of FGF2. Traffic 10, 1765-1772.

Miller, S.A., Johnson, M.L., and Stukenberg, P.T. (2008). Kinetochore attachments require an interaction between unstructured tails on microtubules and $\mathrm{Ndc} 80(\mathrm{Hec} 1)$. Curr Biol 18, 1785-1791.

Miranda, J.J., De Wulf, P., Sorger, P.K., and Harrison, S.C. (2005). The yeast DASH complex forms closed rings on microtubules. Nat Struct Mol Biol 12, 138-143.

Mishra, R.K., Chakraborty, P., Arnaoutov, A., Fontoura, B.M., and Dasso, M. The Nup107-160 complex and gamma-TuRC regulate microtubule polymerization at kinetochores. Nat Cell Biol 12, 164-169.

Mitchison, T., and Kirschner, M. (1984). Dynamic instability of microtubule growth. Nature 312, 237-242. 
Mitchison, T.J., and Kirschner, M.W. (1985). Properties of the kinetochore in vitro. I. Microtubule nucleation and tubulin binding. J Cell Biol 101, 755-765.

Mollinari, C., Reynaud, C., Martineau-Thuillier, S., Monier, S., Kieffer, S., Garin, J., Andreassen, P.R., Boulet, A., Goud, B., Kleman, J.P., et al. (2003). The mammalian passenger protein TD-60 is an RCC1 family member with an essential role in prometaphase to metaphase progression. Dev Cell 5, 295-307.

Momotani, K., Khromov, A.S., Miyake, T., Stukenberg, P.T., and Somlyo, A.V. (2008). Cep57, a multidomain protein with unique microtubule and centrosomal localization domains. Biochem J 412, 265-273.

Moroi, Y., Peebles, C., Fritzler, M.J., Steigerwald, J., and Tan, E.M. (1980). Autoantibody to centromere (kinetochore) in scleroderma sera. Proc Natl Acad Sci U S A 77, 1627-1631.

Musacchio, A., and Salmon, E.D. (2007). The spindle-assembly checkpoint in space and time. Nat Rev Mol Cell Biol 8, 379-393.

Nguyen, H.G., and Ravid, K. (2006). Tetraploidy/aneuploidy and stem cells in cancer promotion: The role of chromosome passenger proteins. J Cell Physiol 208, 12-22.

Nogales, E., and Ramey, V.H. (2009). Structure-function insights into the yeast Dam1 kinetochore complex. J Cell Sci 122, 3831-3836.

Ohta, S., Bukowski-Wills, J.C., Sanchez-Pulido, L., Alves Fde, L., Wood, L., Chen, Z.A., Platani, M., Fischer, L., Hudson, D.F., Ponting, C.P., et al. The protein composition of mitotic chromosomes determined using multiclassifier combinatorial proteomics. Cell $142,810-821$.

Okada, M., Cheeseman, I.M., Hori, T., Okawa, K., McLeod, I.X., Yates, J.R., 3rd, Desai, A., and Fukagawa, T. (2006). The CENP-H-I complex is required for the efficient incorporation of newly synthesized CENP-A into centromeres. Nat Cell Biol 8, 446-457.

Osborne, M.A., Schlenstedt, G., Jinks, T., and Silver, P.A. (1994). Nuf2, a spindle pole body-associated protein required for nuclear division in yeast. J Cell Biol 125, 853-866.

Pagliuca, C., Draviam, V.M., Marco, E., Sorger, P.K., and De Wulf, P. (2009). Roles for the conserved spc105p/kre28p complex in kinetochore-microtubule binding and the spindle assembly checkpoint. PLoS One 4, e7640.

Paintrand, M., Moudjou, M., Delacroix, H., and Bornens, M. (1992). Centrosome organization and centriole architecture: their sensitivity to divalent cations. J Struct Biol $108,107-128$. 
Paweletz, N. (2001). Walther Flemming: pioneer of mitosis research. Nat Rev Mol Cell Biol 2, 72-75.

Peterson, J.B., and Ris, H. (1976). Electron-microscopic study of the spindle and chromosome movement in the yeast Saccharomyces cerevisiae. J Cell Sci 22, 219-242.

Petrovic, A., Pasqualato, S., Dube, P., Krenn, V., Santaguida, S., Cittaro, D., Monzani, S., Massimiliano, L., Keller, J., Tarricone, A., et al. The MIS12 complex is a protein interaction hub for outer kinetochore assembly. J Cell Biol 190, 835-852.

Pfarr, C.M., Coue, M., Grissom, P.M., Hays, T.S., Porter, M.E., and McIntosh, J.R. (1990). Cytoplasmic dynein is localized to kinetochores during mitosis. Nature 345, 263265.

Porter, I.M., McClelland, S.E., Khoudoli, G.A., Hunter, C.J., Andersen, J.S., McAinsh, A.D., Blow, J.J., and Swedlow, J.R. (2007). Bod1, a novel kinetochore protein required for chromosome biorientation. J Cell Biol 179, 187-197.

Powers, A.F., Franck, A.D., Gestaut, D.R., Cooper, J., Gracyzk, B., Wei, R.R., Wordeman, L., Davis, T.N., and Asbury, C.L. (2009). The Ndc80 kinetochore complex forms load-bearing attachments to dynamic microtubule tips via biased diffusion. Cell $136,865-875$.

Przewloka, M.R., Venkei, Z., Bolanos-Garcia, V.M., Debski, J., Dadlez, M., and Glover, D.M. CENP-C is a structural platform for kinetochore assembly. Curr Biol 21, 399-405.

Putkey, F.R., Cramer, T., Morphew, M.K., Silk, A.D., Johnson, R.S., McIntosh, J.R., and Cleveland, D.W. (2002). Unstable kinetochore-microtubule capture and chromosomal instability following deletion of CENP-E. Dev Cell 3, 351-365.

Rahmani, Z., Gagou, M.E., Lefebvre, C., Emre, D., and Karess, R.E. (2009). Separating the spindle, checkpoint, and timer functions of BubR1. J Cell Biol 187, 597-605.

Rieder, C.L. (1982). The formation, structure, and composition of the mammalian kinetochore and kinetochore fiber. Int Rev Cytol 79, 1-58.

Roos, U.P. (1973). Light and electron microscopy of rat kangaroo cells in mitosis. II. Kinetochore structure and function. Chromosoma 41, 195-220.

Rosasco-Nitcher, S.E., Lan, W., Khorasanizadeh, S., and Stukenberg, P.T. (2008). Centromeric Aurora-B activation requires TD-60, microtubules, and substrate priming phosphorylation. Science 319, 469-472.

Rosenfeld, S.S., van Duffelen, M., Behnke-Parks, W.M., Beadle, C., Corrreia, J., and Xing, J. (2009). The ATPase cycle of the mitotic motor CENP-E. J Biol Chem 284, 32858-32868. 
Ruchaud, S., Carmena, M., and Earnshaw, W.C. (2007). Chromosomal passengers: conducting cell division. Nat Rev Mol Cell Biol 8, 798-812.

Ruiz-Miro, M., Colomina, N., Fernandez, R.M., Gari, E., Gallego, C., and Aldea, M. Translokin (Cep57) interacts with cyclin D1 and prevents its nuclear accumulation in quiescent fibroblasts. Traffic 12, 549-562.

Sanchez-Perez, I., Renwick, S.J., Crawley, K., Karig, I., Buck, V., Meadows, J.C., Franco-Sanchez, A., Fleig, U., Toda, T., and Millar, J.B. (2005). The DASH complex and Klp5/Klp6 kinesin coordinate bipolar chromosome attachment in fission yeast. EMBO J 24, 2931-2943.

Santaguida, S., and Musacchio, A. (2009). The life and miracles of kinetochores. EMBO J 28, 2511-2531.

Santaguida, S., Vernieri, C., Villa, F., Ciliberto, A., and Musacchio, A. Evidence that Aurora B is implicated in spindle checkpoint signalling independently of error correction. EMBO J 30, 1508-1519.

Schueler, M.G., Swanson, W., Thomas, P.J., and Green, E.D. Adaptive evolution of foundation kinetochore proteins in primates. Mol Biol Evol 27, 1585-1597.

Screpanti, E., De Antoni, A., Alushin, G.M., Petrovic, A., Melis, T., Nogales, E., and Musacchio, A. Direct binding of Cenp-C to the Mis12 complex joins the inner and outer kinetochore. Curr Biol 21, 391-398.

Shang, C., Hazbun, T.R., Cheeseman, I.M., Aranda, J., Fields, S., Drubin, D.G., and Barnes, G. (2003). Kinetochore protein interactions and their regulation by the Aurora kinase Ipl1p. Mol Biol Cell 14, 3342-3355.

Shima, T., Kon, T., Imamula, K., Ohkura, R., and Sutoh, K. (2006). Two modes of microtubule sliding driven by cytoplasmic dynein. Proc Natl Acad Sci U S A 103, 1773617740.

Sillje, H.H., Nagel, S., Korner, R., and Nigg, E.A. (2006). HURP is a Ran-importin betaregulated protein that stabilizes kinetochore microtubules in the vicinity of chromosomes. Curr Biol 16, 731-742.

Sjoblom, B., Ylanne, J., and Djinovic-Carugo, K. (2008). Novel structural insights into Factin-binding and novel functions of calponin homology domains. Curr Opin Struct Biol 18, 702-708.

Slep, K.C., and Vale, R.D. (2007). Structural basis of microtubule plus end tracking by XMAP215, CLIP-170, and EB1. Mol Cell 27, 976-991. 
Snape, K., Hanks, S., Ruark, E., Barros-Nunez, P., Elliott, A., Murray, A., Lane, A.H., Shannon, N., Callier, P., Chitayat, D., et al. Mutations in CEP57 cause mosaic variegated aneuploidy syndrome. Nat Genet.

Stearns, T., and Kirschner, M. (1994). In vitro reconstitution of centrosome assembly and function: the central role of gamma-tubulin. Cell 76, 623-637.

Stukenberg, P.T., and Foltz, D.R. Kinetochores: orchestrating the chromosomal minuet. Curr Biol 20, R522-525.

Sundin, L.J., Guimaraes, G.J., and Deluca, J.G. The NDC80 complex proteins Nuf2 and Hec1 make distinct contributions to kinetochore-microtubule attachment in mitosis. Mol Biol Cell 22, 759-768.

Tanaka, T.U., and Desai, A. (2008). Kinetochore-microtubule interactions: the means to the end. Curr Opin Cell Biol 20, 53-63.

Tanaka, T.U., Rachidi, N., Janke, C., Pereira, G., Galova, M., Schiebel, E., Stark, M.J., and Nasmyth, K. (2002). Evidence that the Ipl1-Sli15 (Aurora kinase-INCENP) complex promotes chromosome bi-orientation by altering kinetochore-spindle pole connections. Cell 108, 317-329.

Theis, M., Slabicki, M., Junqueira, M., Paszkowski-Rogacz, M., Sontheimer, J., Kittler, R., Heninger, A.K., Glatter, T., Kruusmaa, K., Poser, I., et al. (2009). Comparative profiling identifies C13orf3 as a component of the Ska complex required for mammalian cell division. EMBO J 28, 1453-1465.

Thompson, S.L., and Compton, D.A. (2008). Examining the link between chromosomal instability and aneuploidy in human cells. J Cell Biol 180, 665-672.

Tien, J.F., Umbreit, N.T., Gestaut, D.R., Franck, A.D., Cooper, J., Wordeman, L., Gonen, T., Asbury, C.L., and Davis, T.N. Cooperation of the Dam1 and Ndc80 kinetochore complexes enhances microtubule coupling and is regulated by aurora B. J Cell Biol 189, 713-723.

Tomonaga, T., Matsushita, K., Ishibashi, M., Nezu, M., Shimada, H., Ochiai, T., Yoda, K., and Nomura, F. (2005). Centromere protein H is up-regulated in primary human colorectal cancer and its overexpression induces aneuploidy. Cancer Res 65, 4683-4689.

Tooley, J.G., Miller, S.A., and Stukenberg, P.T. The Ndc80 complex uses a tripartite attachment point to couple microtubule depolymerization to chromosome movement. Mol Biol Cell 22, 1217-1226.

Uchida, K.S., Takagaki, K., Kumada, K., Hirayama, Y., Noda, T., and Hirota, T. (2009). Kinetochore stretching inactivates the spindle assembly checkpoint. J Cell Biol 184, 383390. 
Vader, G., Medema, R.H., and Lens, S.M. (2006). The chromosomal passenger complex: guiding Aurora-B through mitosis. J Cell Biol 173, 833-837.

VandenBeldt, K.J., Barnard, R.M., Hergert, P.J., Meng, X., Maiato, H., and McEwen, B.F. (2006). Kinetochores use a novel mechanism for coordinating the dynamics of individual microtubules. Curr Biol 16, 1217-1223.

Vergnolle, M.A., and Taylor, S.S. (2007). Cenp-F links kinetochores to Ndel1/Nde1/Lis1/dynein microtubule motor complexes. Curr Biol 17, 1173-1179.

Vorozhko, V.V., Emanuele, M.J., Kallio, M.J., Stukenberg, P.T., and Gorbsky, G.J. (2008). Multiple mechanisms of chromosome movement in vertebrate cells mediated through the Ndc80 complex and dynein/dynactin. Chromosoma 117, 169-179.

Wan, X., O'Quinn, R.P., Pierce, H.L., Joglekar, A.P., Gall, W.E., DeLuca, J.G., Carroll, C.W., Liu, S.T., Yen, T.J., McEwen, B.F., et al. (2009). Protein architecture of the human kinetochore microtubule attachment site. Cell 137, 672-684.

Wang, H.W., Long, S., Ciferri, C., Westermann, S., Drubin, D., Barnes, G., and Nogales, E. (2008). Architecture and flexibility of the yeast Ndc80 kinetochore complex. J Mol Biol 383, 894-903.

Wei, R.R., Al-Bassam, J., and Harrison, S.C. (2007). The Ndc80/HEC1 complex is a contact point for kinetochore-microtubule attachment. Nat Struct Mol Biol 14, 54-59.

Wei, R.R., Schnell, J.R., Larsen, N.A., Sorger, P.K., Chou, J.J., and Harrison, S.C. (2006). Structure of a central component of the yeast kinetochore: the Spc24p/Spc25p globular domain. Structure 14, 1003-1009.

Wei, R.R., Sorger, P.K., and Harrison, S.C. (2005). Molecular organization of the Ndc80 complex, an essential kinetochore component. Proc Natl Acad Sci U S A 102, 5363 5367.

Weiss, E., and Winey, M. (1996). The Saccharomyces cerevisiae spindle pole body duplication gene MPS1 is part of a mitotic checkpoint. J Cell Biol 132, 111-123.

Welburn, J.P., and Cheeseman, I.M. (2008). Toward a molecular structure of the eukaryotic kinetochore. Dev Cell 15, 645-655.

Welburn, J.P., Grishchuk, E.L., Backer, C.B., Wilson-Kubalek, E.M., Yates, J.R., 3rd, and Cheeseman, I.M. (2009). The human kinetochore Ska1 complex facilitates microtubule depolymerization-coupled motility. Dev Cell 16, 374-385.

Welburn, J.P., Vleugel, M., Liu, D., Yates, J.R., 3rd, Lampson, M.A., Fukagawa, T., and Cheeseman, I.M. Aurora B phosphorylates spatially distinct targets to differentially regulate the kinetochore-microtubule interface. Mol Cell 38, 383-392. 
Westermann, S., Avila-Sakar, A., Wang, H.W., Niederstrasser, H., Wong, J., Drubin, D.G., Nogales, E., and Barnes, G. (2005). Formation of a dynamic kinetochoremicrotubule interface through assembly of the Dam1 ring complex. Mol Cell 17, 277290.

Westermann, S., Wang, H.W., Avila-Sakar, A., Drubin, D.G., Nogales, E., and Barnes, G. (2006). The Dam1 kinetochore ring complex moves processively on depolymerizing microtubule ends. Nature 440, 565-569.

White, R.A., Pan, Z., and Salisbury, J.L. (2000). GFP-centrin as a marker for centriole dynamics in living cells. Microsc Res Tech 49, 451-457.

Wigge, P.A., Jensen, O.N., Holmes, S., Soues, S., Mann, M., and Kilmartin, J.V. (1998). Analysis of the Saccharomyces spindle pole by matrix-assisted laser desorption/ionization (MALDI) mass spectrometry. J Cell Biol 141, 967-977.

Wigge, P.A., and Kilmartin, J.V. (2001). The Ndc80p complex from Saccharomyces cerevisiae contains conserved centromere components and has a function in chromosome segregation. J Cell Biol 152, 349-360.

Williams, B.C., Li, Z., Liu, S., Williams, E.V., Leung, G., Yen, T.J., and Goldberg, M.L. (2003). Zwilch, a new component of the ZW10/ROD complex required for kinetochore functions. Mol Biol Cell 14, 1379-1391.

Wilson-Kubalek, E.M., Cheeseman, I.M., Yoshioka, C., Desai, A., and Milligan, R.A. (2008). Orientation and structure of the Ndc80 complex on the microtubule lattice. J Cell Biol 182, 1055-1061.

Wojcik, E., Basto, R., Serr, M., Scaerou, F., Karess, R., and Hays, T. (2001). Kinetochore dynein: its dynamics and role in the transport of the Rough deal checkpoint protein. Nat Cell Biol 3, 1001-1007.

Wong, J., and Fang, G. (2006). HURP controls spindle dynamics to promote proper interkinetochore tension and efficient kinetochore capture. J Cell Biol 173, 879-891.

Wong, J., Lerrigo, R., Jang, C.Y., and Fang, G. (2008). Aurora A regulates the activity of HURP by controlling the accessibility of its microtubule-binding domain. Mol Biol Cell 19, 2083-2091.

Wordeman, L., Steuer, E.R., Sheetz, M.P., and Mitchison, T. (1991). Chemical subdomains within the kinetochore domain of isolated CHO mitotic chromosomes. J Cell Biol 114, 285-294.

Wuhr, M., Chen, Y., Dumont, S., Groen, A.C., Needleman, D.J., Salic, A., and Mitchison, T.J. (2008). Evidence for an upper limit to mitotic spindle length. Curr Biol $18,1256-1261$. 
Yen, T.J., Li, G., Schaar, B.T., Szilak, I., and Cleveland, D.W. (1992). CENP-E is a putative kinetochore motor that accumulates just before mitosis. Nature 359, 536-539.

Zhai, Y., Kronebusch, P.J., and Borisy, G.G. (1995). Kinetochore microtubule dynamics and the metaphase-anaphase transition. J Cell Biol 131, 721-734.

Zimniak, T., Stengl, K., Mechtler, K., and Westermann, S. (2009). Phosphoregulation of the budding yeast EB1 homologue Bim1p by Aurora/Ipl1p. J Cell Biol 186, 379-391.

Zinkowski, R.P., Meyne, J., and Brinkley, B.R. (1991). The centromere-kinetochore complex: a repeat subunit model. J Cell Biol 113, 1091-1110.

Zuccolo, M., Alves, A., Galy, V., Bolhy, S., Formstecher, E., Racine, V., Sibarita, J.B., Fukagawa, T., Shiekhattar, R., Yen, T., et al. (2007). The human Nup107-160 nuclear pore subcomplex contributes to proper kinetochore functions. EMBO J 26, 1853-1864. 
Appendix: Plasmids Maps 


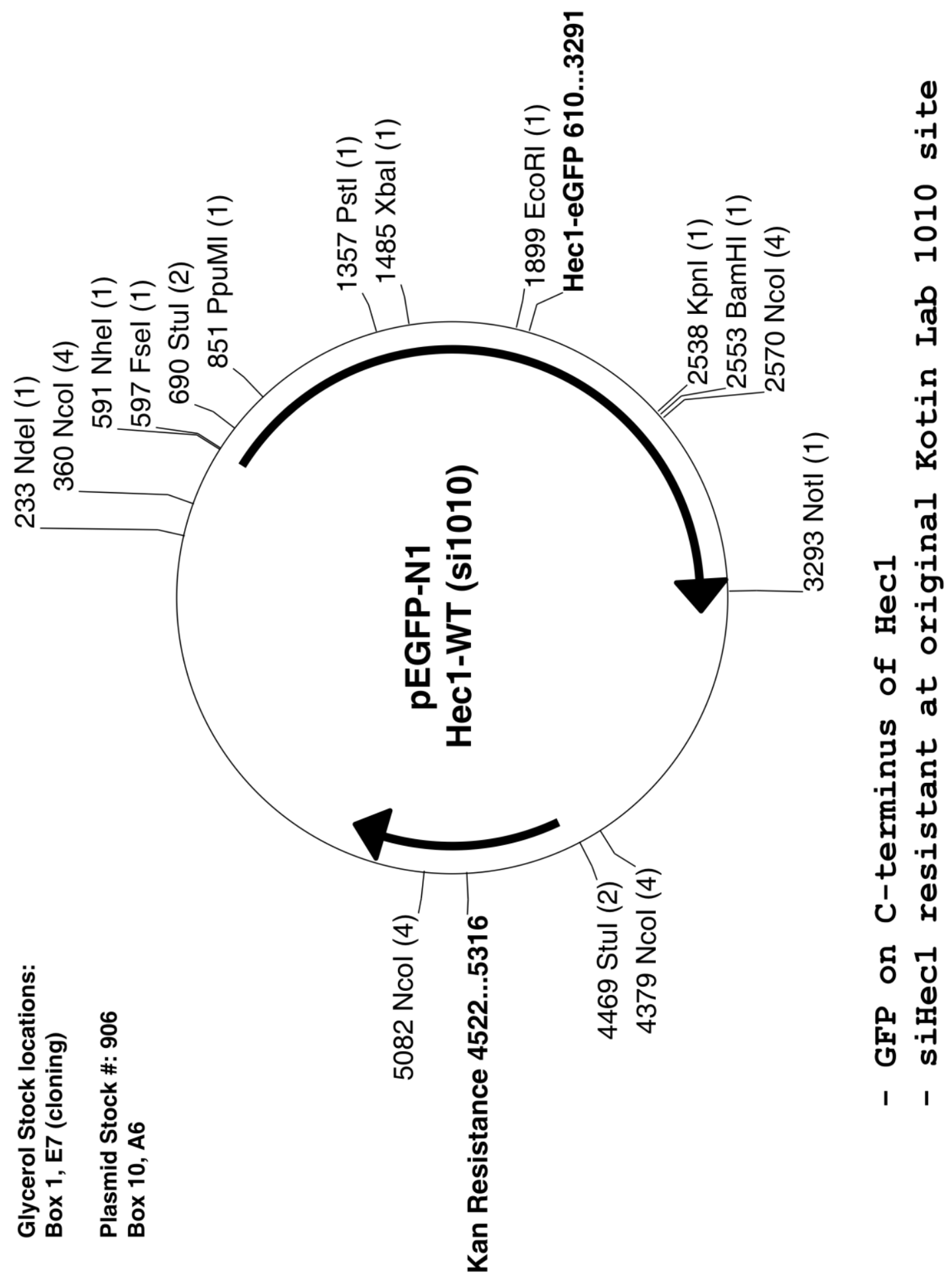




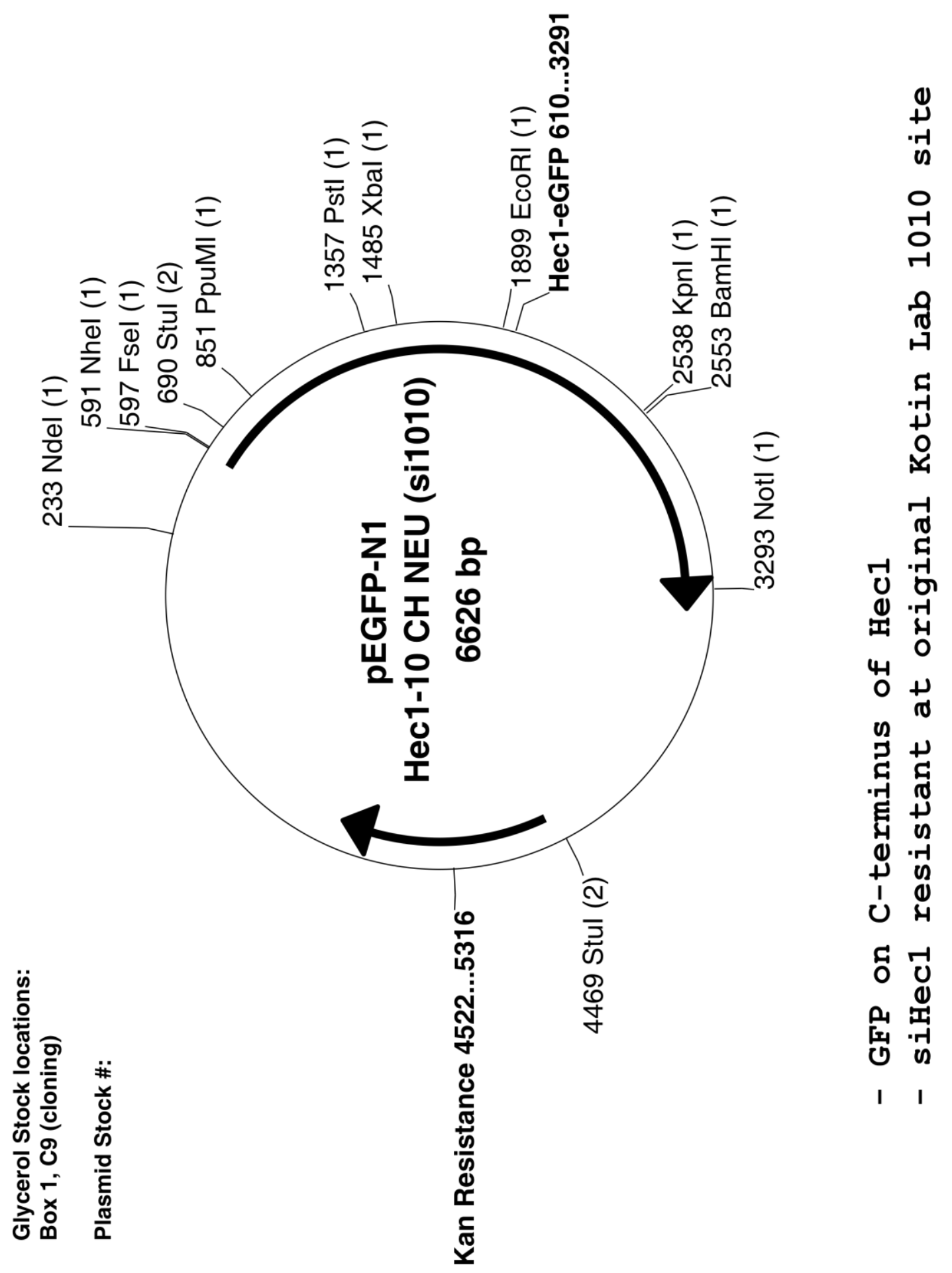




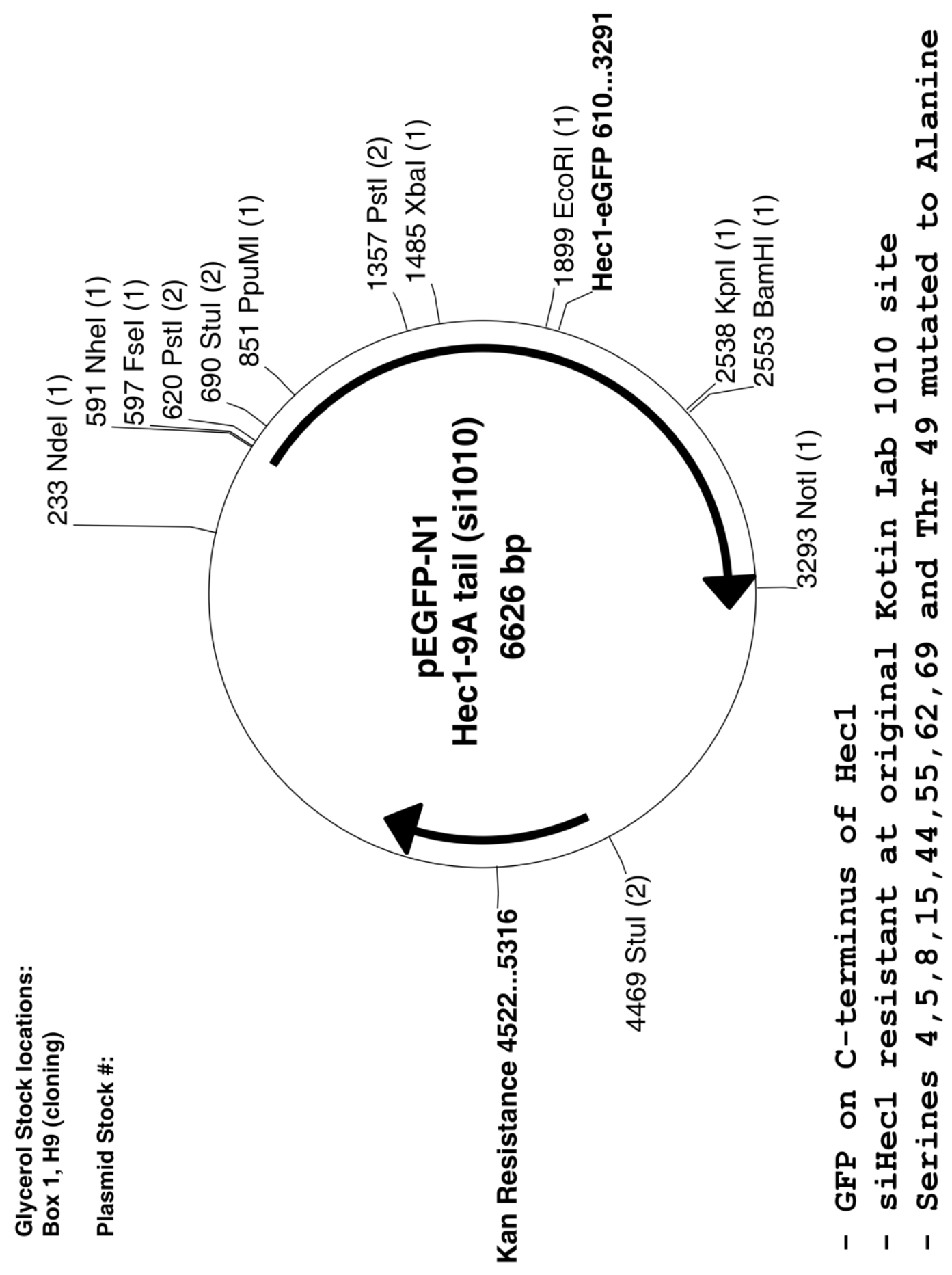




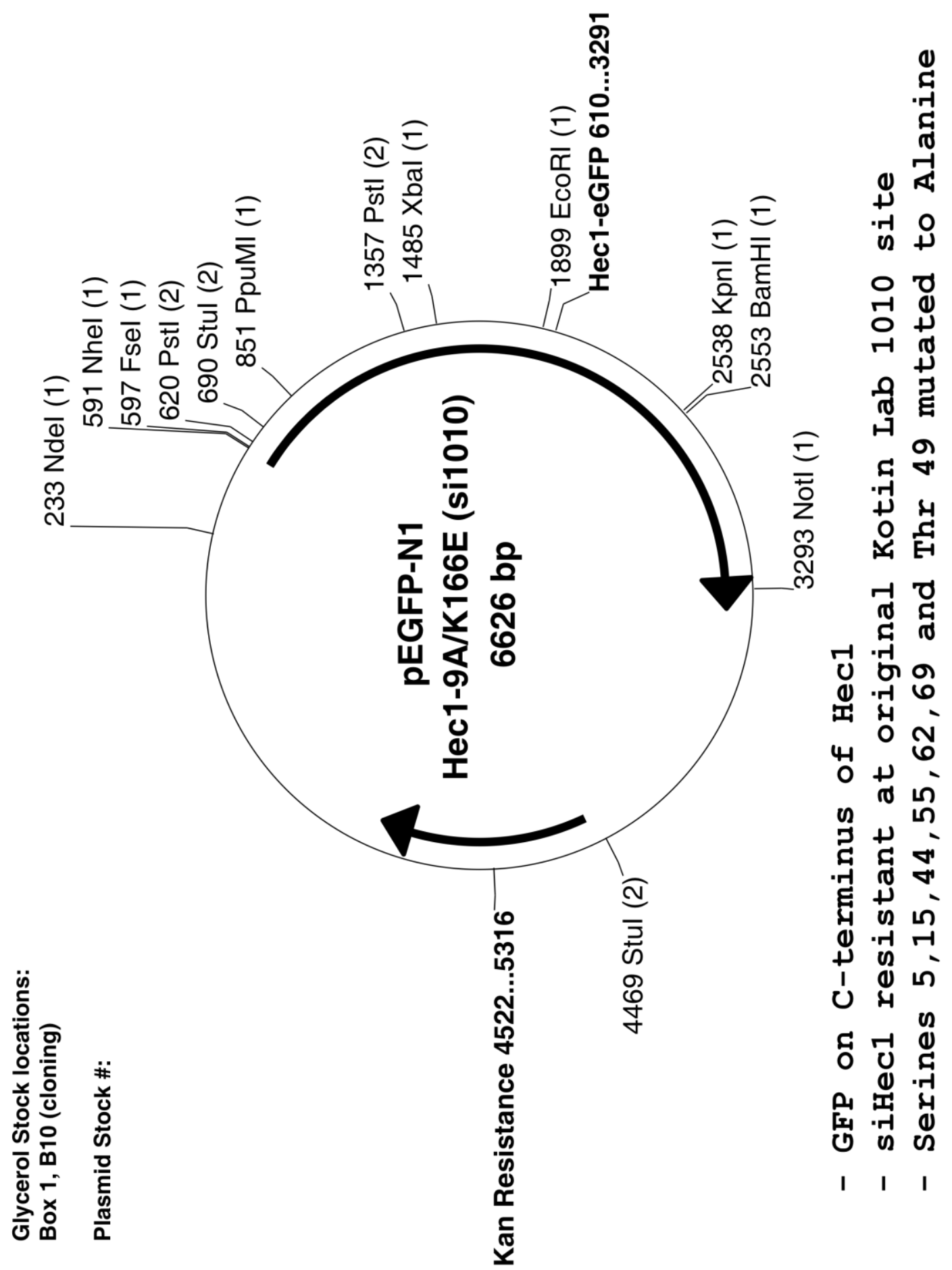

Other Hec1-9A plasmids:9A/K89E, 9A/K115E, 9A/K89EK166E 


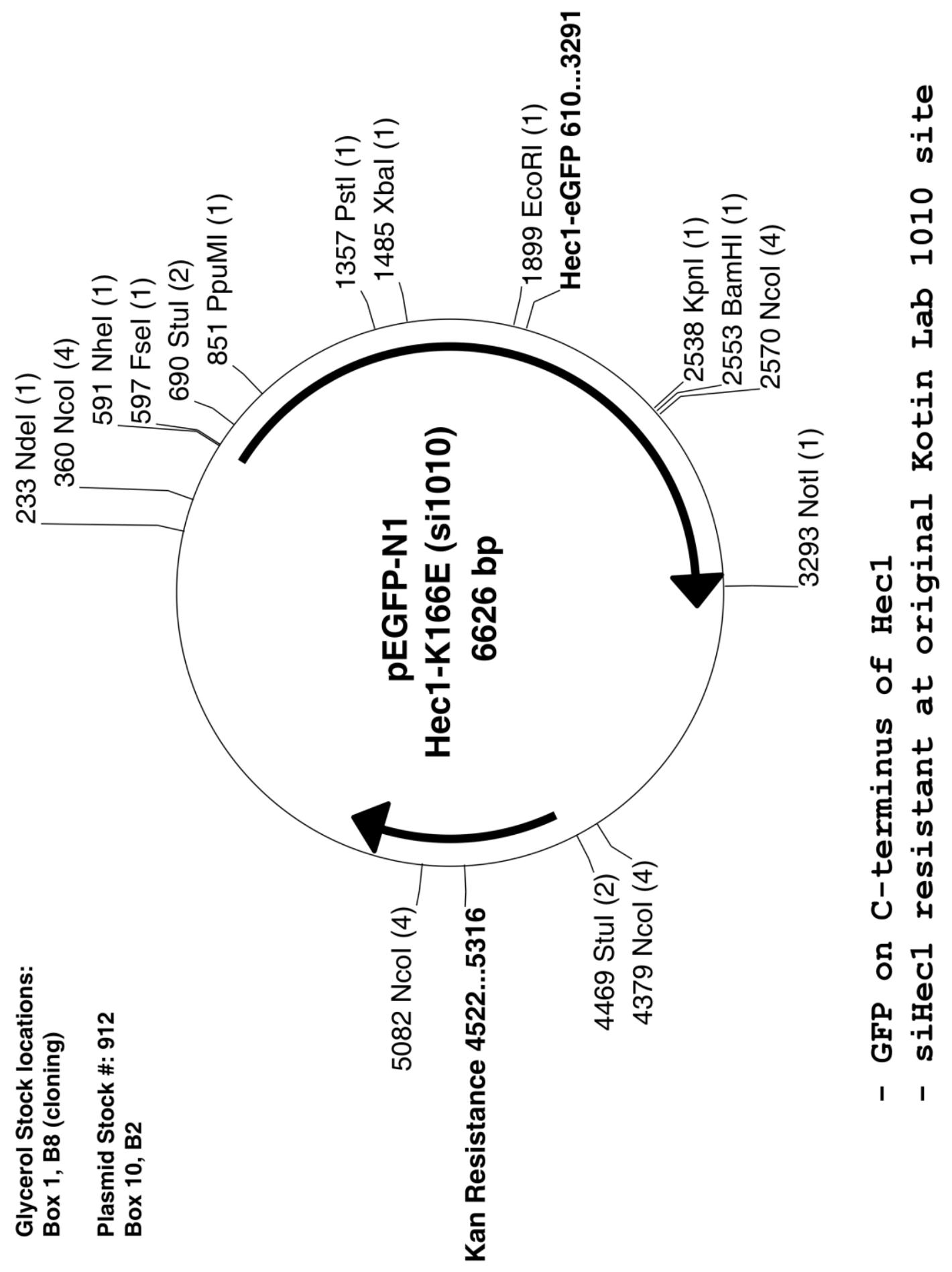

Similar Hec1 mutants: K89E, K115E, K123E 


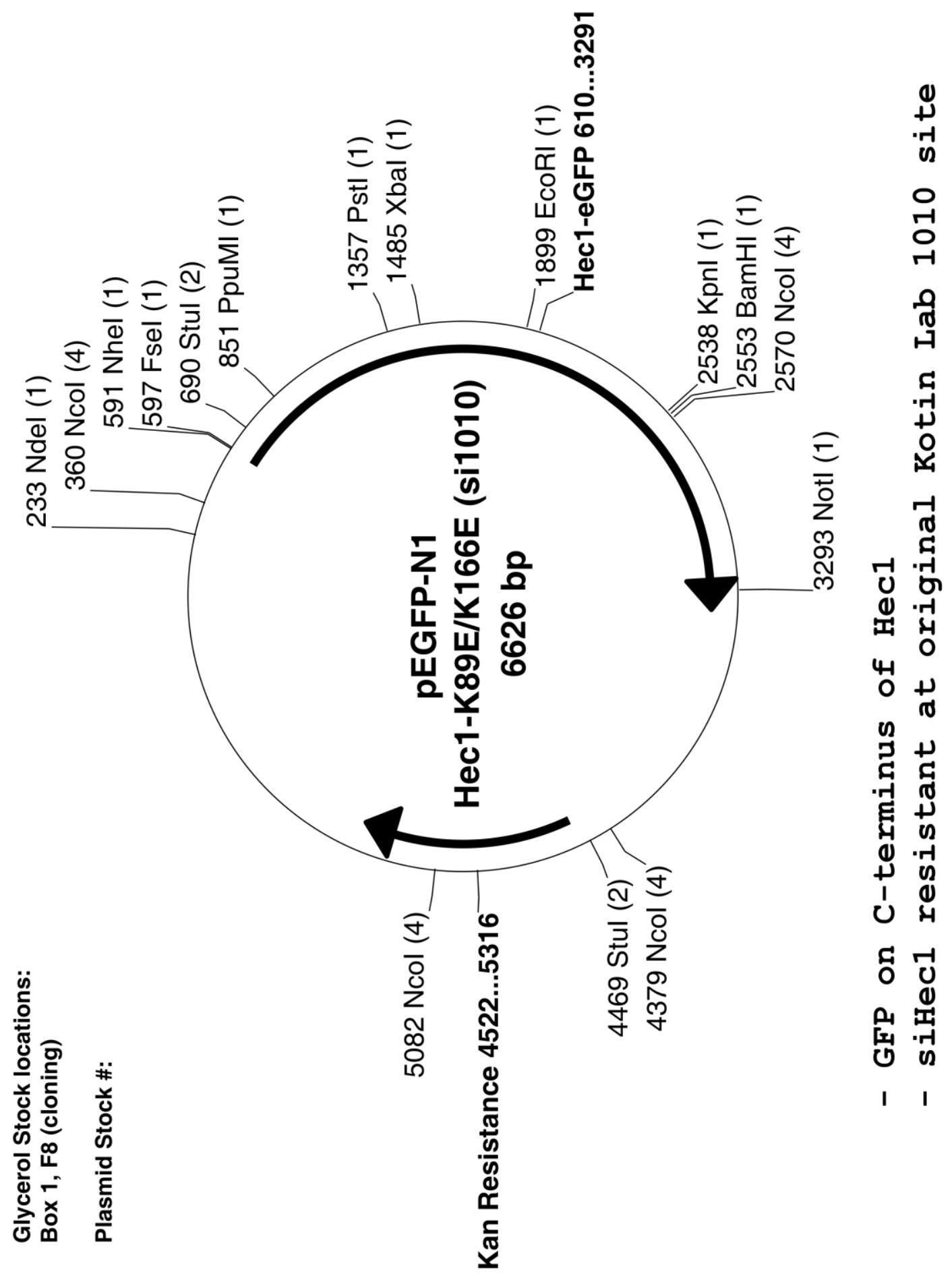

Similar Hec1 mutants: K89E/K115E, K89E/K123E 


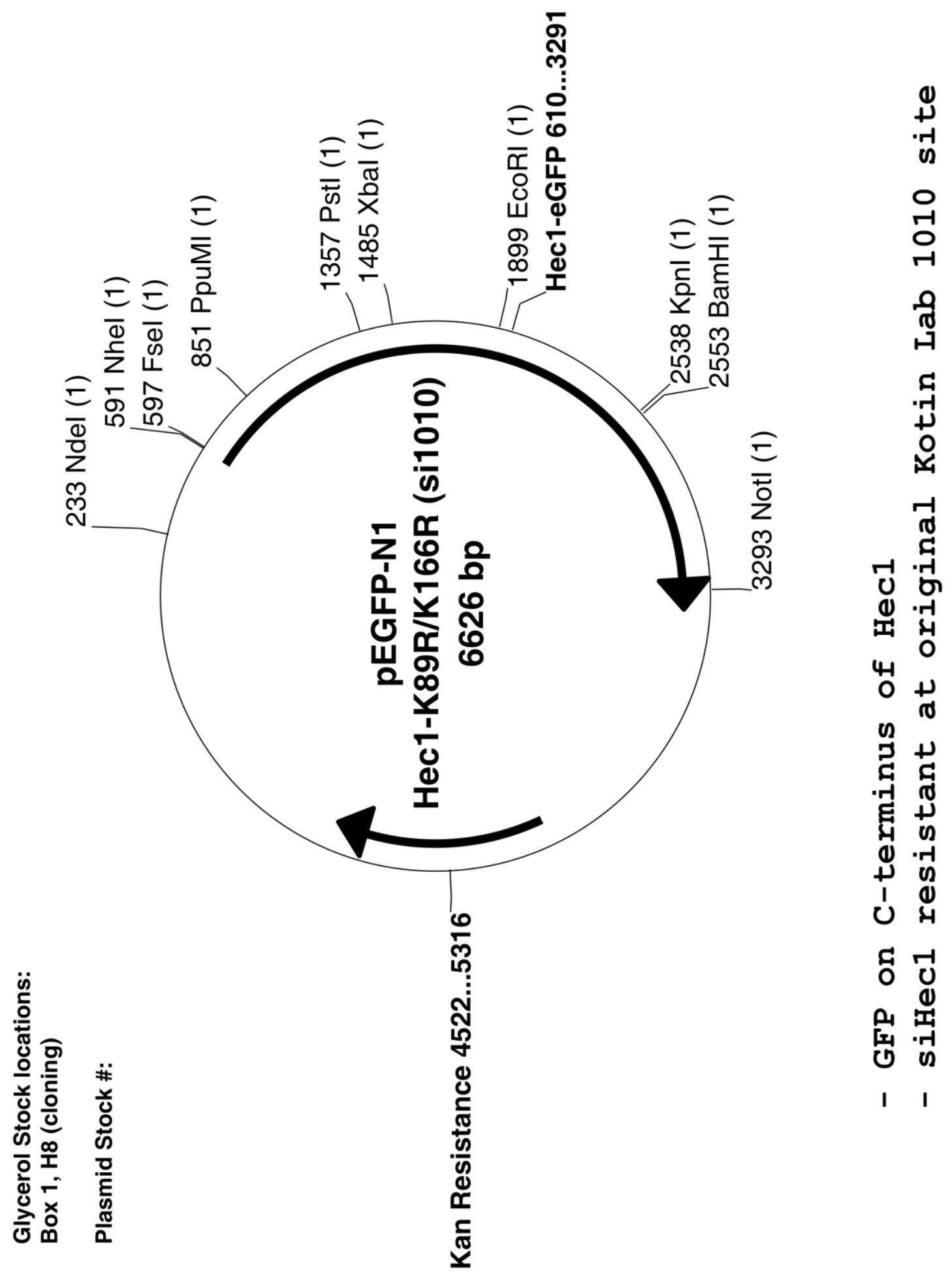

Similar Hec1 mutants: K166R, K89AK166A, K89A, K166A, K89A/115A 


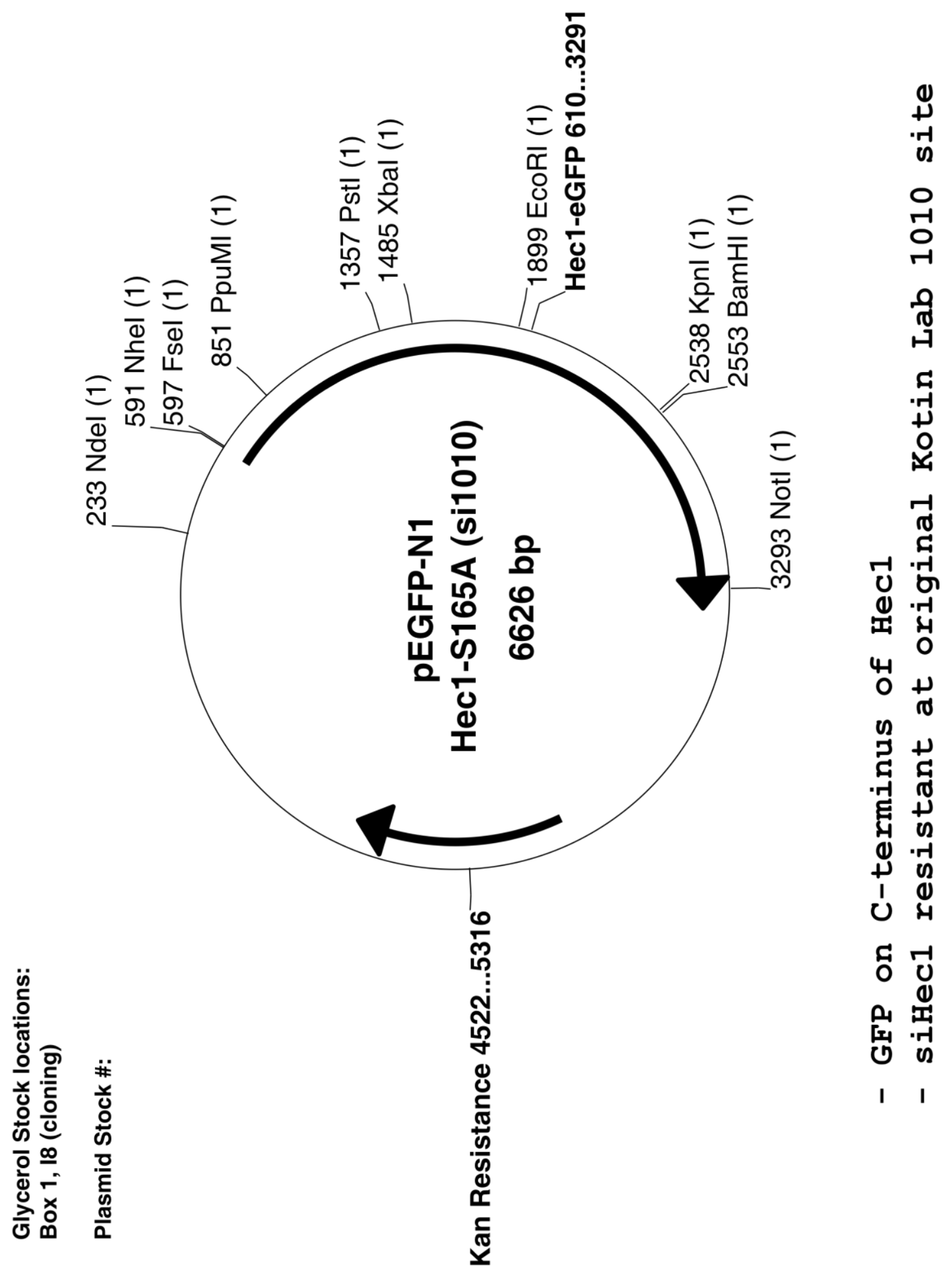

Similar Hec1 mutants: S165E 


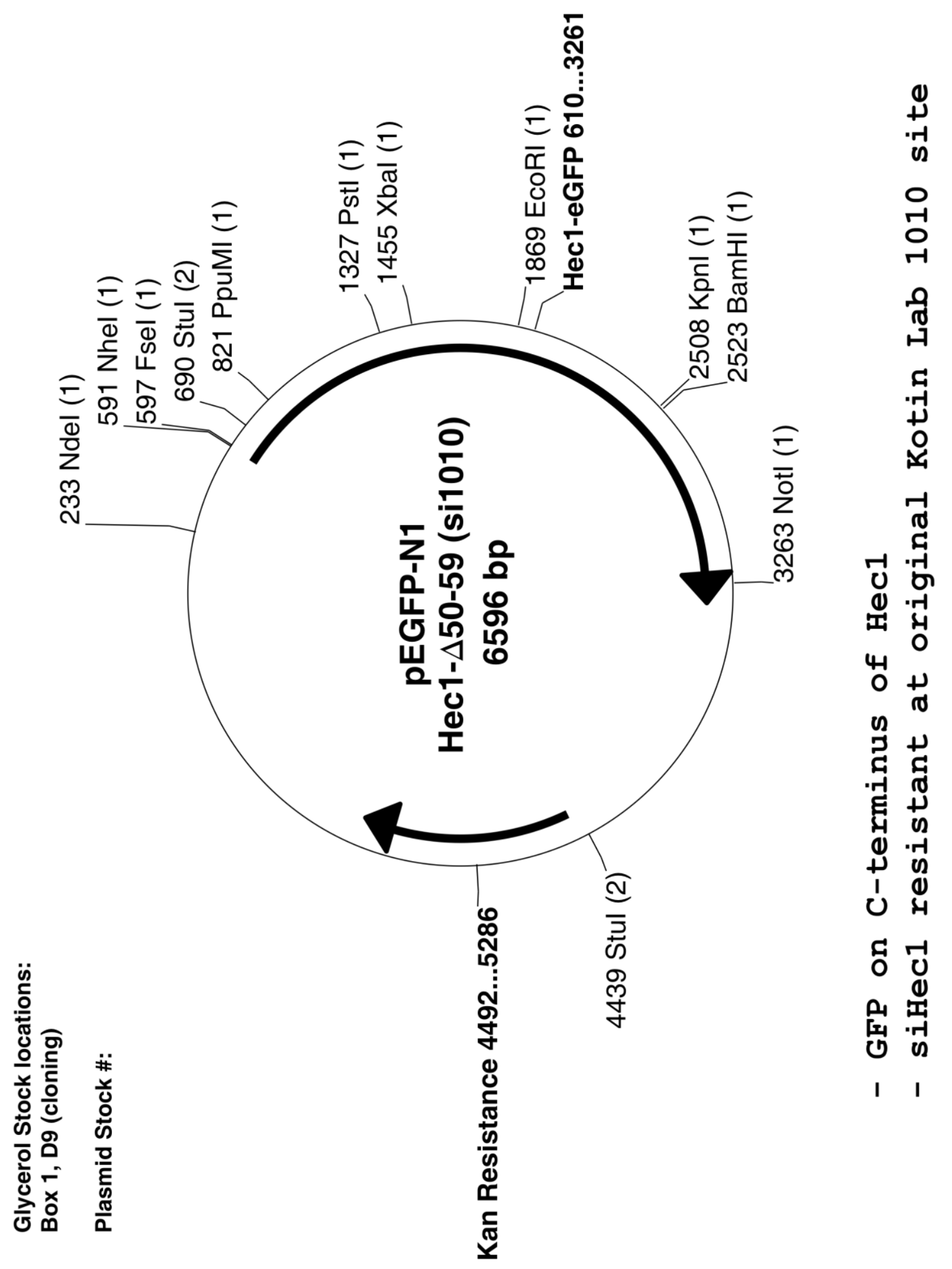




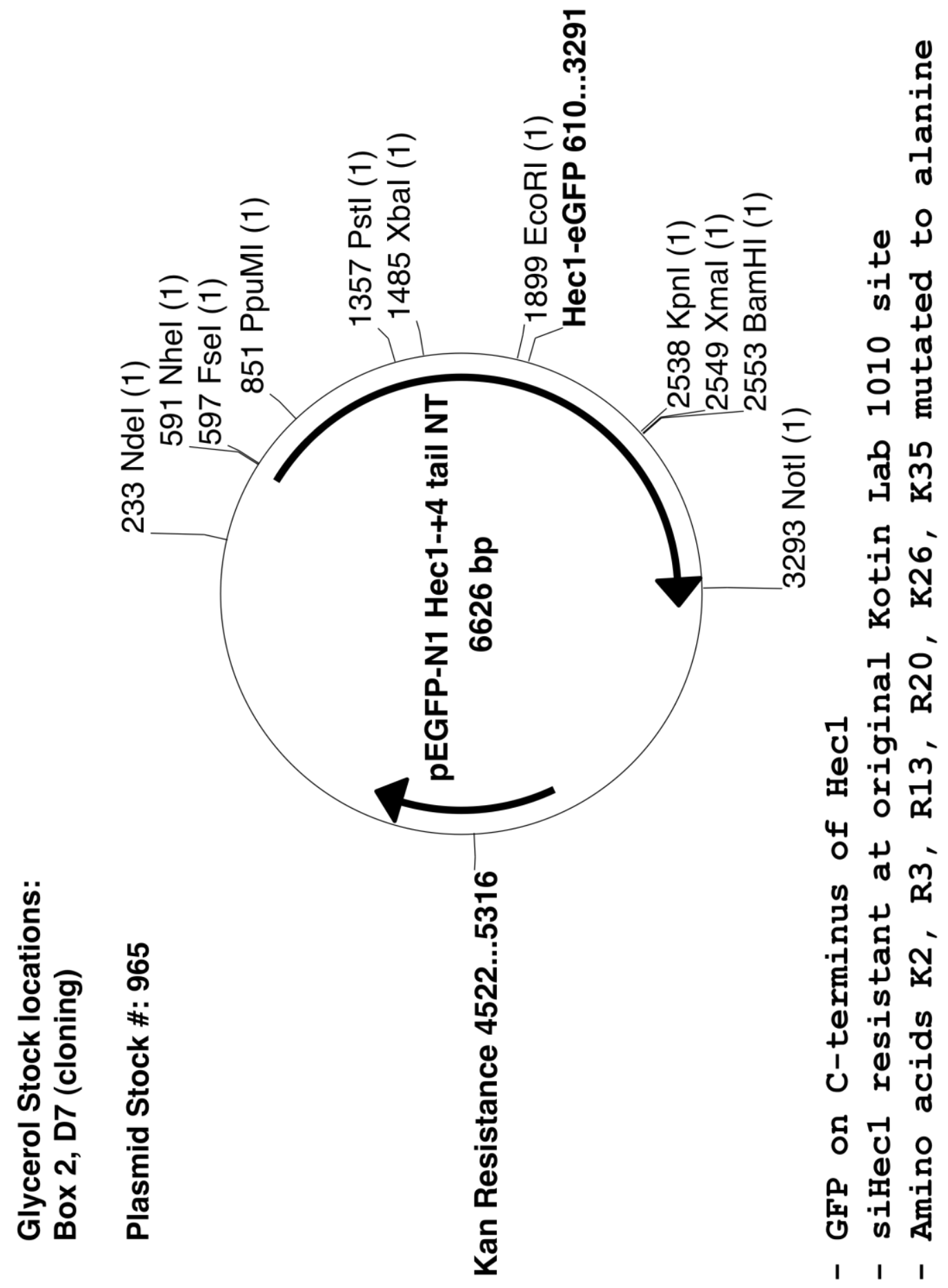




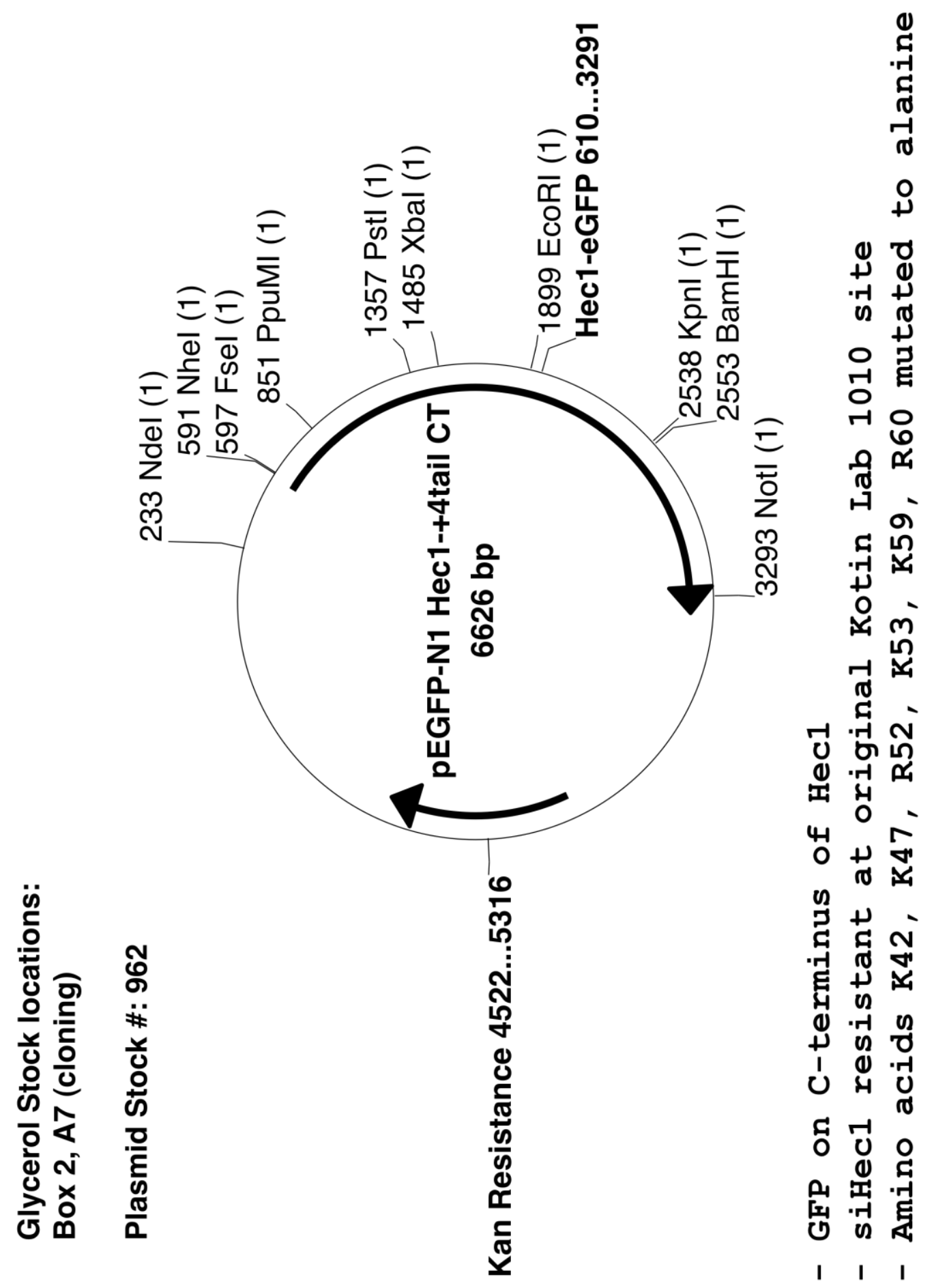




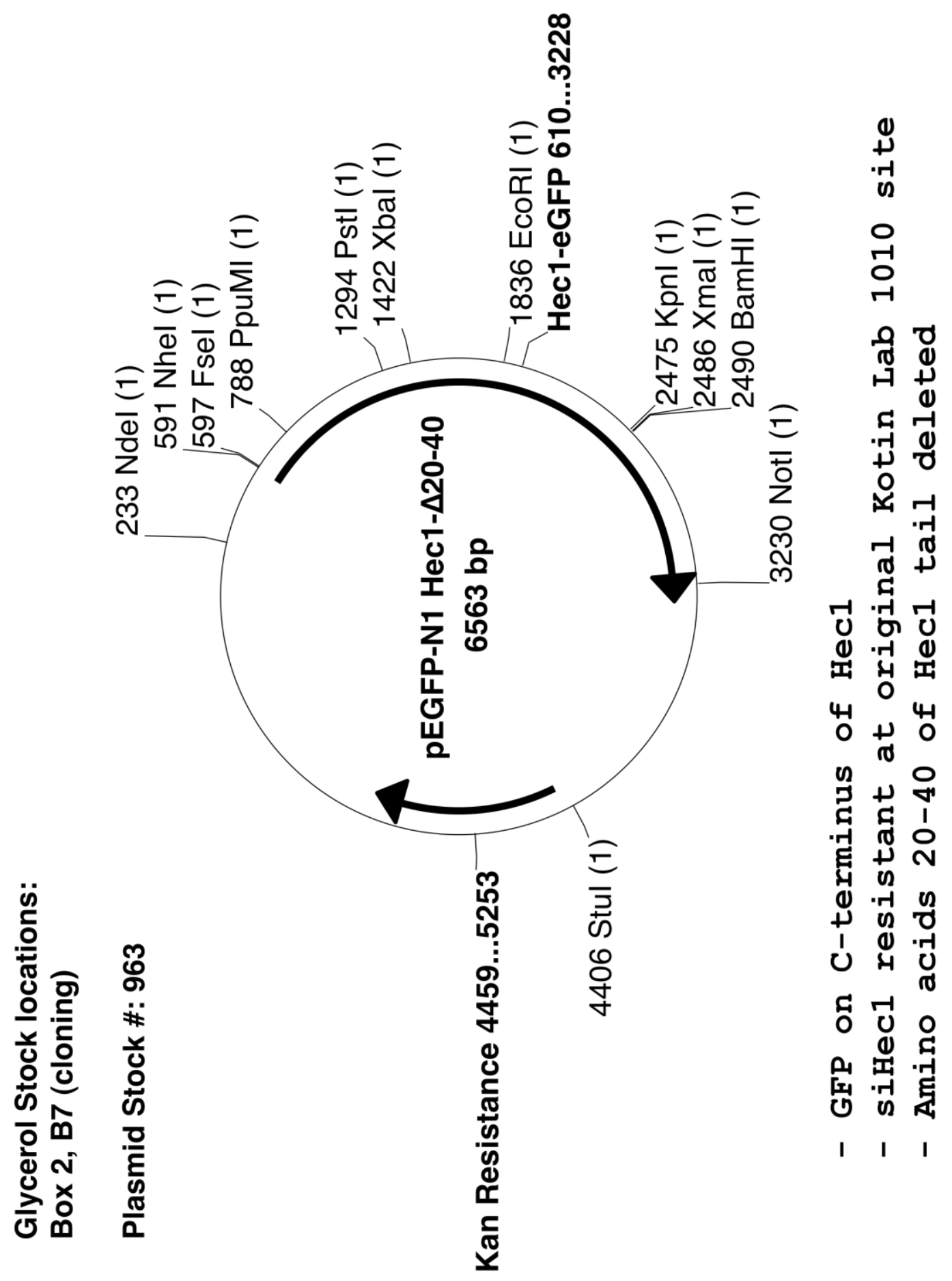

Similar Hec1 mutants: $\Delta 40-60, \Delta 60-80, \Delta 40-80$ 


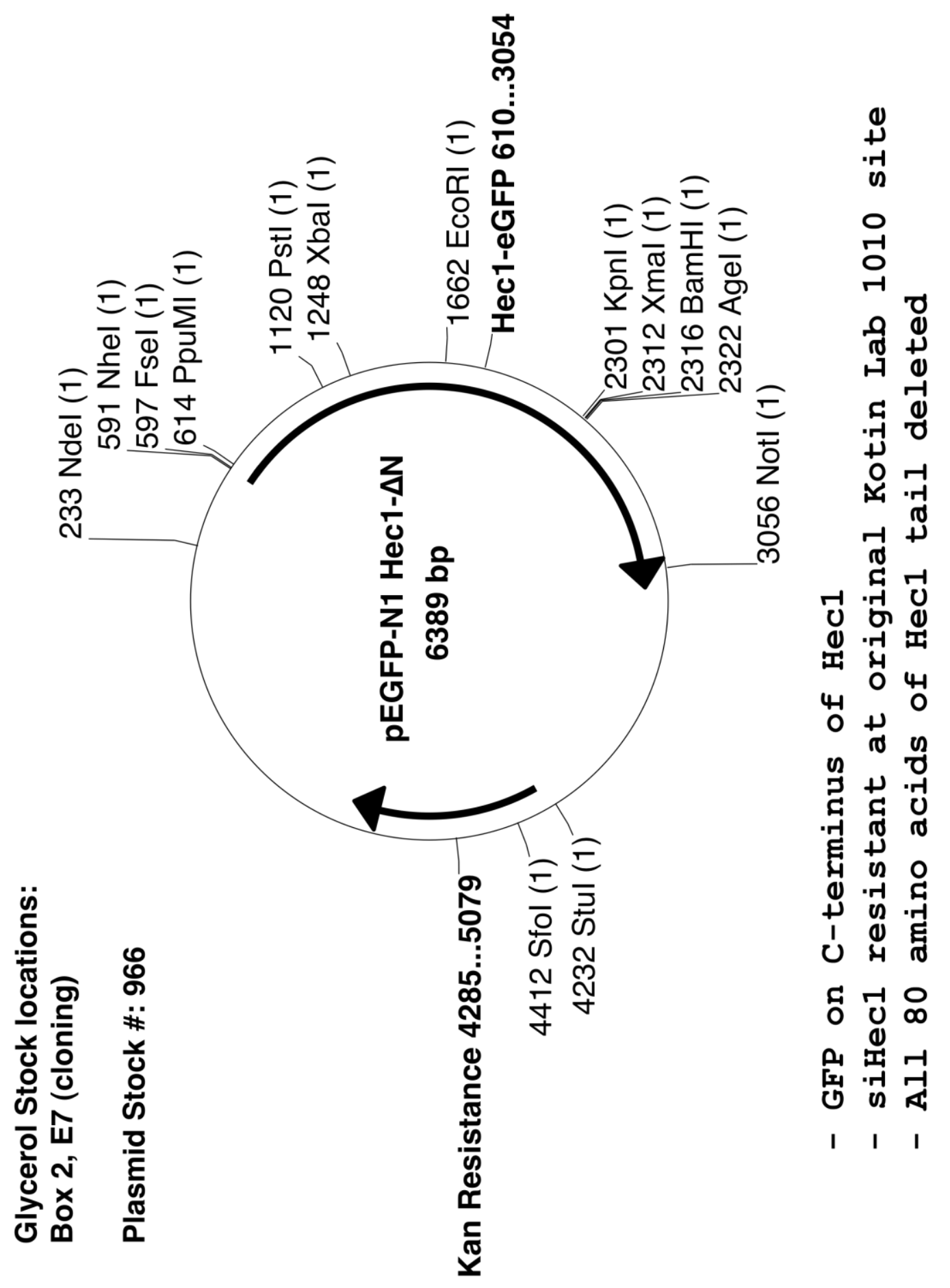




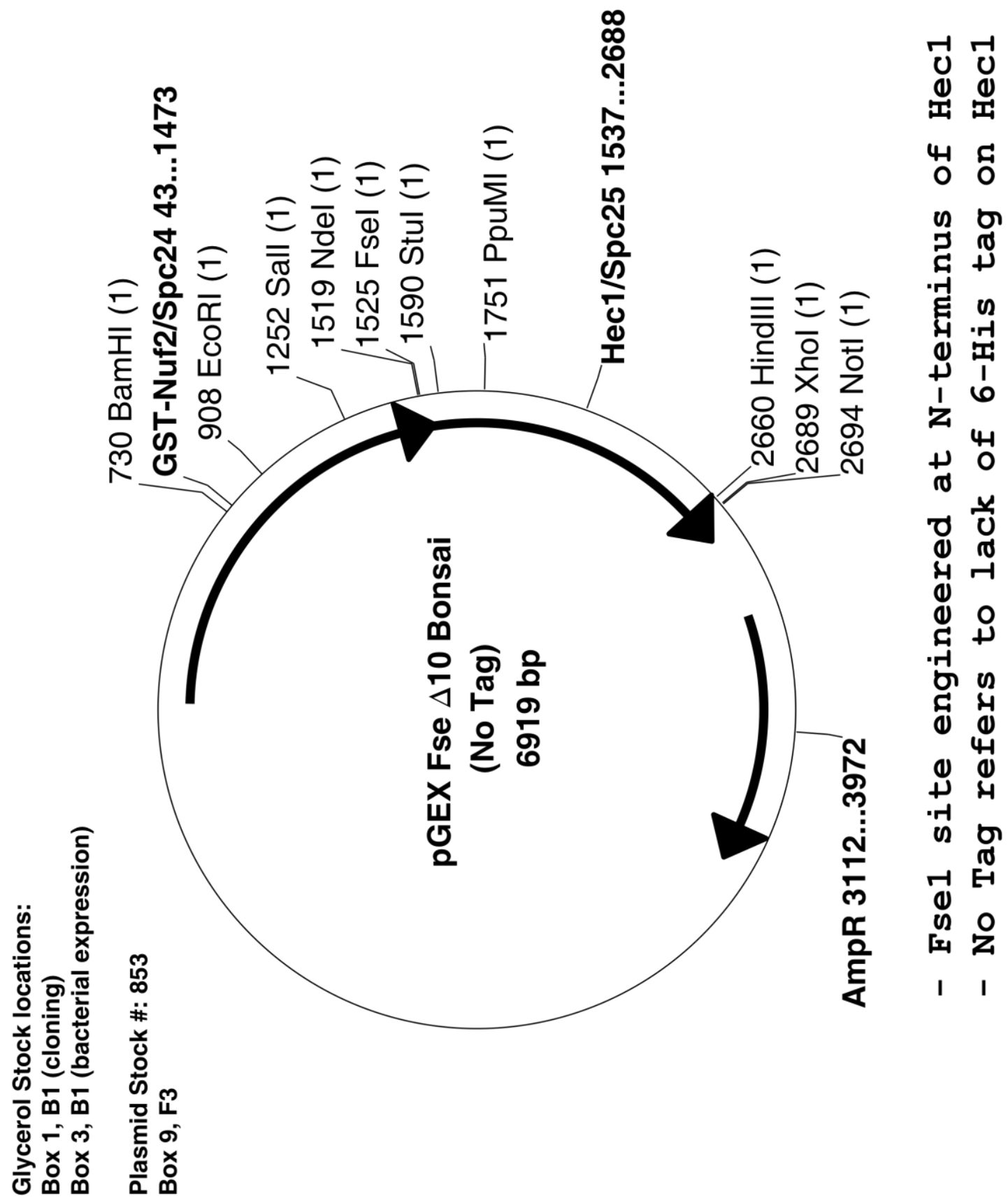

Similar Hec1 mutants: $\Delta 20,30,40,50,60,70,80(N)$ 


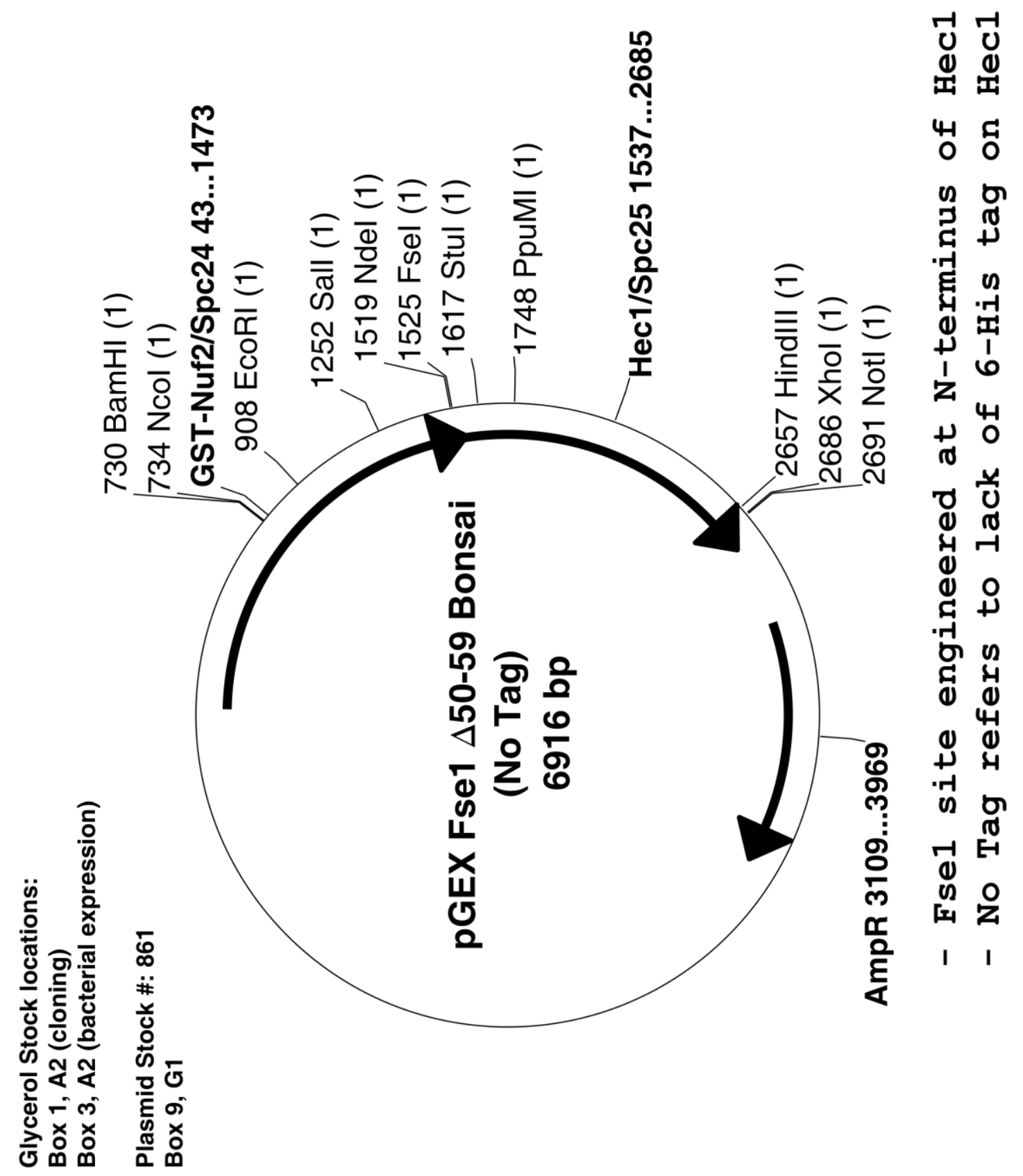




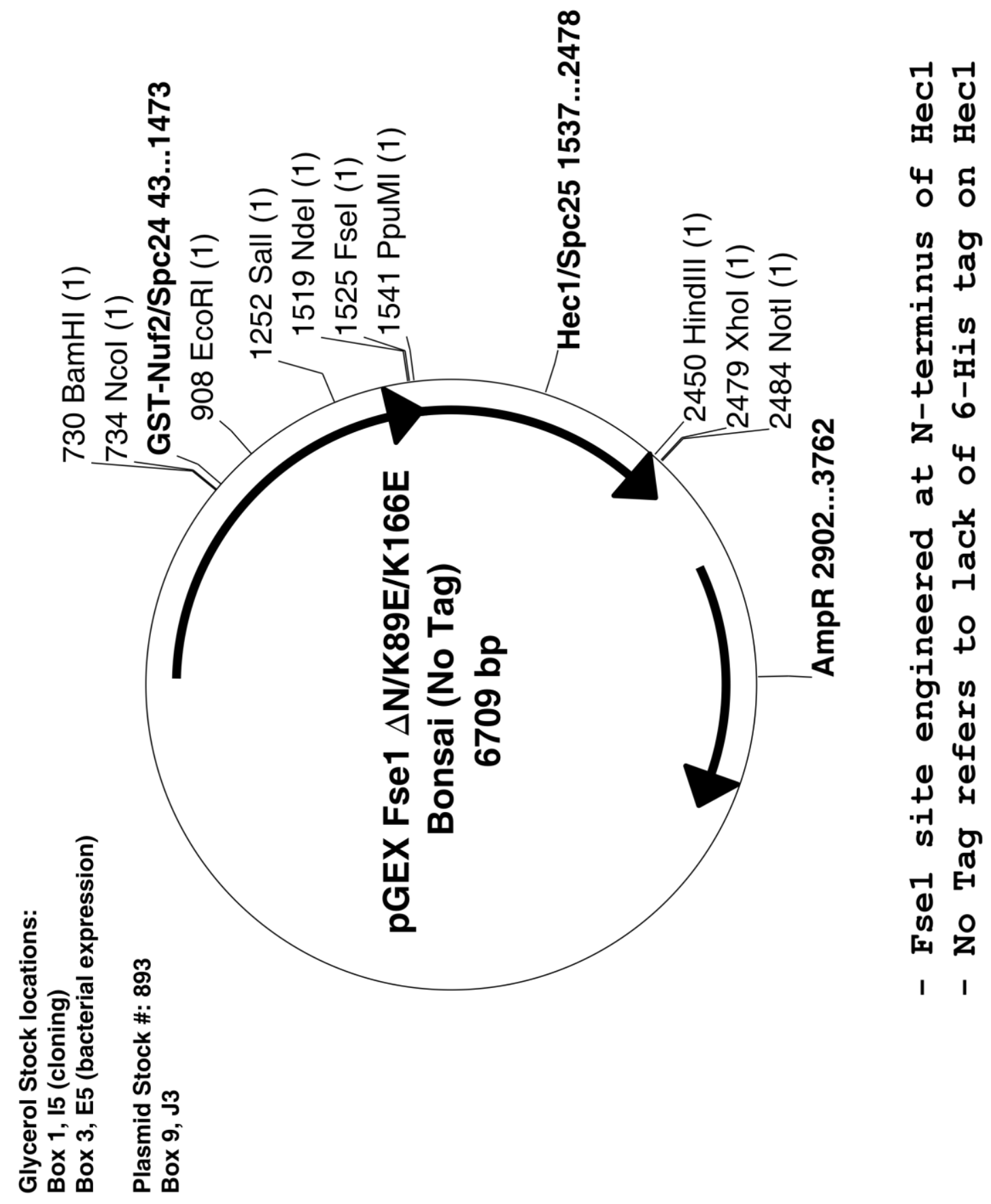

Similar Bonsai mutants: K115E, K166E 
